\title{
चISAS
}

In cooperation with the U.S. Environmental Protection Agency

Water-Quality, Bed-Sediment, and Biological Data (October 2004 through September 2005) and Statistical Summaries of Data for Streams in the Upper Clark Fork Basin, Montana

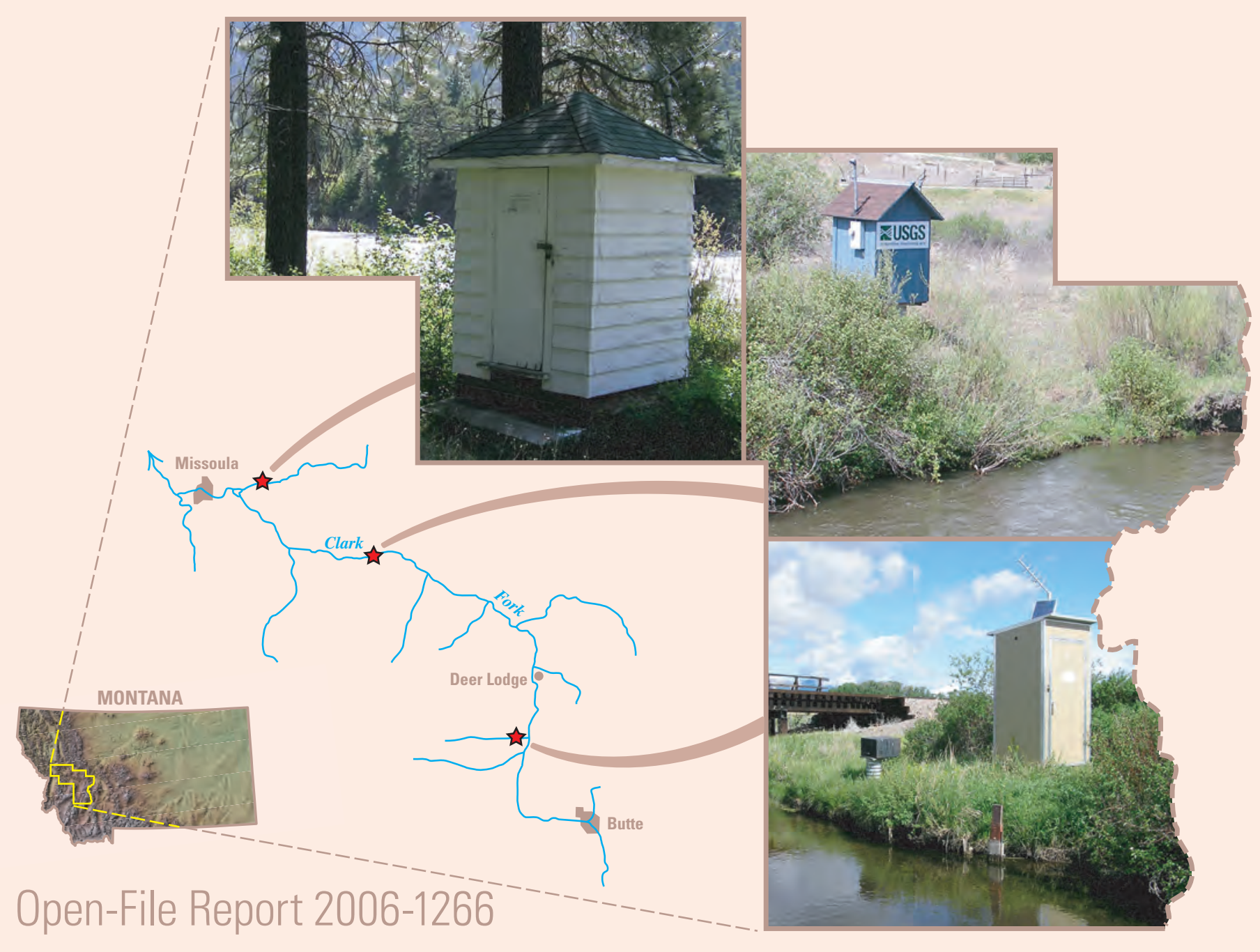

U.S. Department of the Interior

U.S. Geological Survey 
COVER PHOTOGRAPHS: Banner: Lost Creek near Galen, Mont. (12323850).

Left: Gage house at Blackfoot River near Bonner, Mont. (12340000).

Center: Gage house at Clark Fork near Drummond, Mont. (12331800).

Lower: Gage house at Lost Creek near Galen, Mont. (12323850).

Photographs by Kent A. Dodge, U.S. Geological Survey, taken in the spring of 2006. 


\section{Water-Quality, Bed-Sediment, and Biological Data (October 2004 through September 2005) and Statistical Summaries of Data for Streams in the Upper Clark Fork Basin, Montana}

By Kent A. Dodge, Michelle I. Hornberger, and Jessica L. Dyke

Open-File Report 2006-1266 


\section{U.S. Department of the Interior DIRK KEMPTHORNE, Secretary}

\section{U.S. Geological Survey \\ Mark D. Myers, Director}

\section{U.S. Geological Survey, Reston, Virginia: 2006}

For product and ordering information:

World Wide Web: http://www.usgs.gov/pubprod

Telephone: 1-888-ASK-USGS

For more information on the USGS — the Federal source for science about the Earth, its natural and living resources, natural hazards, and the environment:

World Wide Web: http://www.usgs.gov

Telephone: 1-888-ASK-USGS

Any use of trade, product, or firm names is for descriptive purposes only and does not imply endorsement by the U.S. Government.

Although this report is in the public domain, permission must be secured from the individual copyright owners to reproduce any copyrighted materials contained within this report.

Suggested citation:

Dodge, K.A., Hornberger, M.I., and Dyke, J.L., 2006, Water-quality, bed-sediment, and biological data (October 2004 through September 2005) and statistical summaries of data for streams in the upper Clark Fork basin, Montana: U.S. Geological Survey Open-File Report 2006-1266, 109 p. 


\section{Contents}

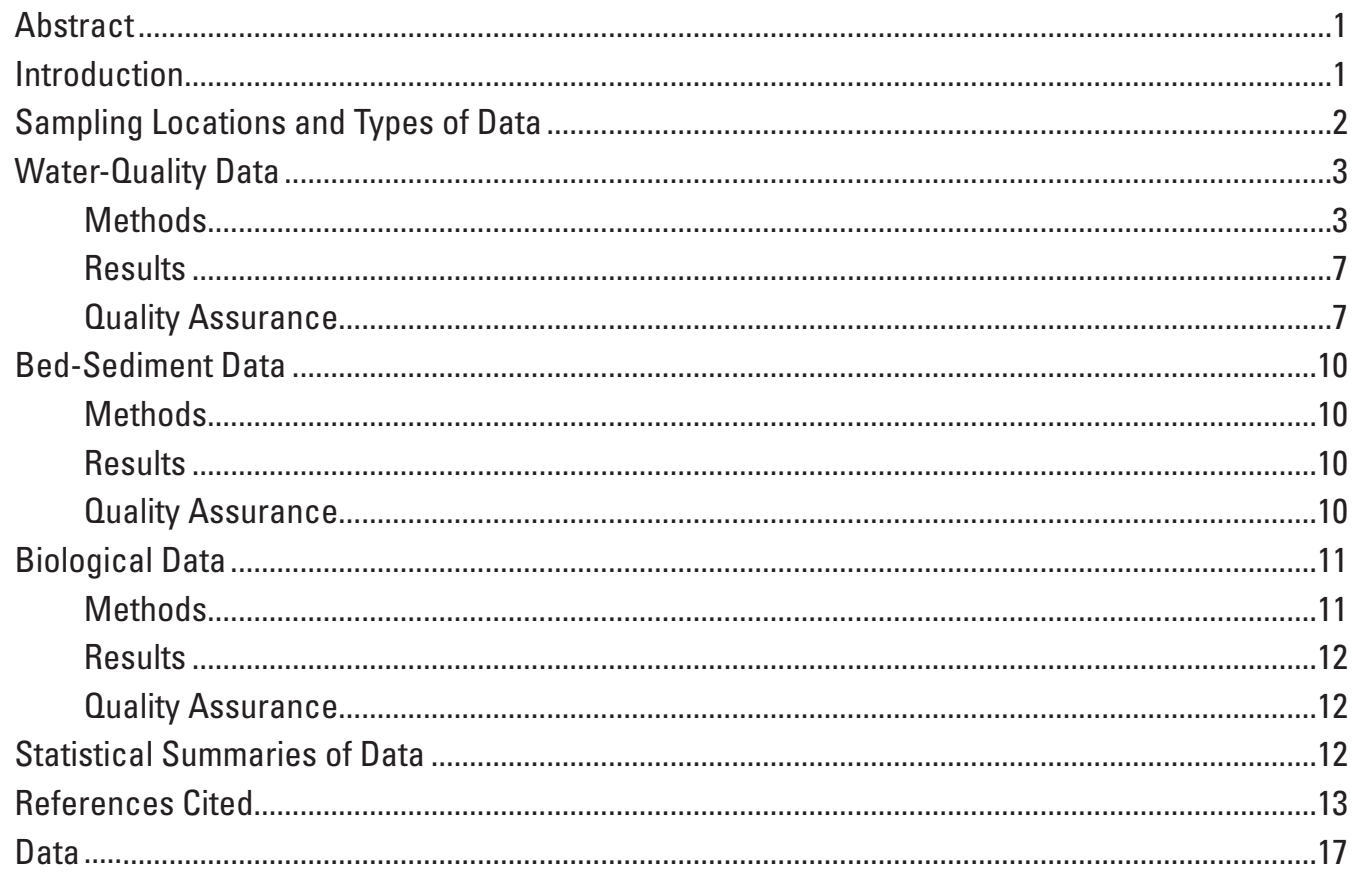

\section{Figure}

1. Map showing location of study area

\section{Tables}

1. Type and period of data collection at sampling sites in the upper Clark Fork basin, Montana

2. Properties measured onsite and constituents analyzed in samples of water, bed sediment, and biota from the upper Clark Fork basin, Montana..........................................6

3. Data-quality objectives for analyses of water-quality samples collected in the upper Clark Fork basin, Montana.

4. Water-quality data for the upper Clark Fork basin, Montana, October 2004 through September 2005.

5-7. Daily mean streamflow and suspended-sediment data, October 2004 through September 2005, for:

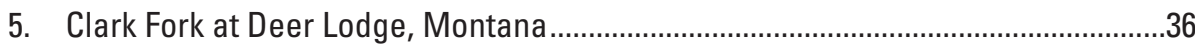

6. Clark Fork at Turah Bridge, near Bonner, Montana ..............................................40

7. Clark Fork above Missoula, Montana......................................................................4

8. Analyses of field replicates for water samples, upper Clark Fork basin, Montana ........48

9. Precision of analyses of field replicates for water samples, upper Clark Fork basin, Montana 
10. Precision of analyses of laboratory replicates for water samples, upper Clark Fork basin, Montana .

11. Recovery efficiency for analyses of laboratory-spiked deionized-water blanks ............52

12. Recovery efficiency for analyses of laboratory-spiked stream samples, upper

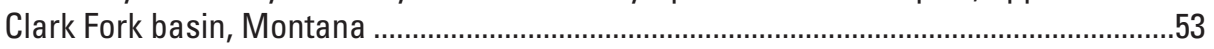

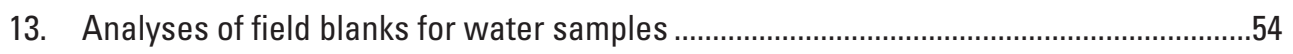

14. Analyses of fine-grained bed sediment, upper Clark Fork basin, Montana, August 2005.

15. Recovery efficiency for analyses of standard reference materials for bed

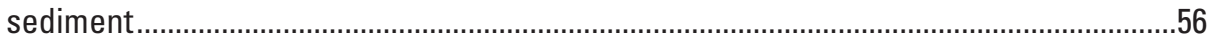

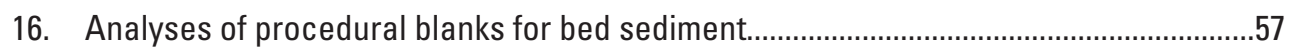

17. Analyses of biota, upper Clark Fork basin, Montana, August 2005 ..................................58

18. Recovery efficiency for analyses of standard reference material for biota ...................60

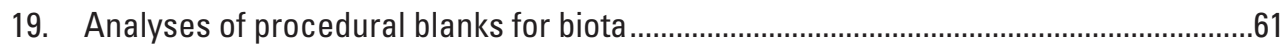

20. Statistical summary of water-quality data for the upper Clark Fork basin, Montana, March 1985 through September 2005

21. Statistical summary of fine-grained bed-sediment data for the upper Clark Fork basin, Montana, August 1986 through August 2005 80

22. Statistical summary of biological data for the upper Clark Fork basin, Montana, August 1986 through August 2005 86

\section{Conversion Factors, Datum, Abbreviated water-quality units, and Acronyms}

\begin{tabular}{lcl}
\hline Multiply & By & To obtain \\
\hline acre-foot (acre-ft) & 1,233 & cubic meter $\left(\mathrm{m}^{3}\right)$ \\
cubic foot per second $\left(\mathrm{ft}^{3} / \mathrm{s}\right)$ & 0.02832 & cubic meter per second $\left(\mathrm{m}^{3} / \mathrm{s}\right)$ \\
foot $(\mathrm{ft})$ & 0.3048 & meter $(\mathrm{m})$ \\
gallon (gal) & 3.785 & liter $(\mathrm{L})$ \\
gallon (gal) & 3,785 & milliliter $(\mathrm{mL})$ \\
inch (in.) & 25.4 & millimeter $(\mathrm{mm})$ \\
inch (in.) & 25,400 & micrometer $(\mu \mathrm{m})$ \\
mile (mi) & 1.609 & kilometer $(\mathrm{km})$ \\
ounce (oz) & 28.35 & gram $(\mathrm{g})$ \\
part per million $(\mathrm{ppm})$ & 1 & microgram per gram $(\mu \mathrm{g} / \mathrm{g})$ \\
square mile (mi $)$ & 2.59 & square kilometer $\left(\mathrm{km}{ }^{2}\right)$ \\
ton per day (ton/d) & 907.2 & kilogram per day $(\mathrm{kg} / \mathrm{d})$ \\
\hline
\end{tabular}

Temperature in degrees Celsius $\left({ }^{\circ} \mathrm{C}\right)$ may be converted to degrees Fahrenheit $\left({ }^{\circ} \mathrm{F}\right)$ as follows:

$$
{ }^{\circ} \mathrm{F}=\left(1.8 \mathrm{x}^{\circ} \mathrm{C}\right)+32
$$

Vertical coordinate information is referenced to the National Geodetic Vertical Datum of 1929 (NGVD 29). Horizontal coordinate information is referenced to the North American Datum of 1927 (NAD 27). 


\section{Water year definition:}

Water year is the 12-month period from 0 ctober 1 through September 30 of the following calendar year. The water year is designated by the calendar year in which it ends. For example, water year 2005 is the period from October 1, 2004, through September 30, 2005.

\section{Abbreviated water-quality units used in this report:}

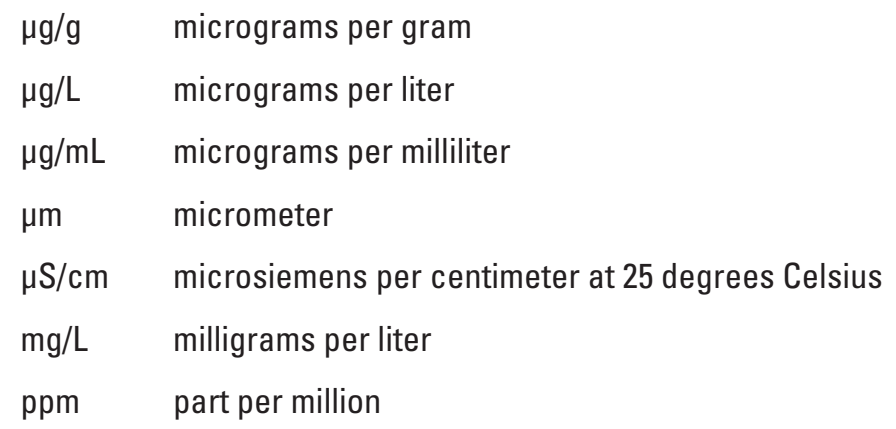

\section{Acronyms used in this report:}

ICAPES inductively coupled argon plasma-emission spectroscopy

LRL laboratory reporting level

LT-MDL long-term method detection level

NWQL USGS National Water Quality Laboratory, Denver, Colo.

RSD relative standard deviation

spp. $\quad$ species

SRM standard reference material

USGS U.S. Geological Survey 


\title{
Water-Quality, Bed-Sediment, and Biological Data (October 2004 through September 2005) and Statistical Summaries of Data for Streams in the Upper Clark Fork Basin, Montana
}

\author{
By Kent A. Dodge, Michelle I. Hornberger ${ }^{1}$, and Jessica L. Dyke ${ }^{1}$
}

\section{Abstract}

Water, bed sediment, and biota were sampled in streams from Butte to below Missoula as part of a long-term monitoring program, conducted in cooperation with the U.S. Environmental Protection Agency, to characterize aquatic resources in the upper Clark Fork basin of western Montana. Sampling sites were located on the Clark Fork, six major tributaries, and three smaller tributaries. Water-quality samples were collected periodically at 18 sites during October 2004 through September 2005 (water year 2005). Bed-sediment and biological samples were collected once in August 2005. The primary constituents analyzed were trace elements associated with tailings from historical mining and smelting activities. This report summarizes the results of water-quality, bed-sediment, and biota samples collected in water year 2005 and provides statistical summaries of data collected since 1985 .

Water-quality data for samples collected periodically from streams include concentrations of selected major ions, trace elements, and suspended sediment. Daily values of suspended-sediment concentration and suspended-sediment discharge were determined for three sites. Bed-sediment data include trace-element concentrations in the fine-grained fraction. Biological data include trace-element concentrations in whole-body tissue of aquatic benthic insects. Qualityassurance data are reported for analytical results of water, bed sediment, and biota. Statistical summaries of waterquality, bed-sediment, and biological data are provided for the period of record since 1985 for each site.

\section{Introduction}

The Clark Fork originates near Warm Springs in western Montana at the confluence of Silver Bow and Warm Springs Creeks (fig. 1). Along the 148-mi reach of stream from Silver

${ }^{1}$ U.S Geological Survey, Menlo Park, Calif.
Bow Creek in Butte to the Clark Fork at Milltown Reservoir, six major tributaries enter: Blacktail Creek, Warm Springs Creek, Little Blackfoot River, Flint Creek, Rock Creek, and Blackfoot River. Principal surface-water uses in the 6,000- $\mathrm{mi}^{2}$ Clark Fork basin above Missoula include irrigation, stock watering, light industry, hydroelectric power generation, and habitat for trout fisheries. Current land uses primarily are cattle production, logging, mining, residential development, and recreation. Large-scale mining and smelting were prevalent land uses in the upper basin for more than one hundred years, but are now either discontinued or substantially smaller in scale.

Deposits of copper, gold, silver, and lead ores were extensively mined, milled, and smelted in the drainages of Silver Bow and Warm Springs Creeks from about 1870 to 1980. Moderate- and small-scale mining also occurred in the basins of most of the major tributaries to the upper Clark Fork. Tailings derived from past mineral processing commonly contain large quantities of trace elements such as arsenic, cadmium, copper, lead, and zinc. Tailings have been eroded, mixed with stream sediment, transported downstream, and deposited in stream channels, on flood plains, and in the Warm Springs Ponds and Milltown Reservoir. The widely dispersed tailings continue to be re-eroded, transported, and redeposited along the stream channel and flood plain, especially during high flows. The occurrence of elevated trace-element concentrations in water and bed sediment can pose a potential risk to aquatic biota and human health.

Concern about the potential toxicity of trace elements to aquatic biota and human health has resulted in a comprehensive effort by State, Federal, and private entities to characterize the aquatic resources in the upper Clark Fork basin to guide and monitor remedial cleanup activities. A long-term data base was considered necessary to detect trends over time in order to evaluate the effectiveness of remediation. Water-quality data have been collected by the U.S. Geological Survey (USGS) at selected sites in the upper Clark Fork basin since 1985 (Lambing, 1987, 1988, 1989, 1990, 1991; Lambing and others, 1994, 1995; and Dodge and others, 1996, 1997, 1998, 1999, 
2000, 2001, 2002, 2003, 2004, 2005). Trace-element data for bed sediment and biota (aquatic benthic insects) have been collected intermittently since 1986 at selected sites as part of studies on bed-sediment contamination and bioaccumulation of metals conducted by the USGS National Research Program (Axtmann and Luoma, 1991; Cain and others, 1992, 1995; Axtmann and others, 1997; Hornberger and others, 1997). In March 1993, an expanded sampling program for water, bed sediment, and biota was implemented by the USGS in cooperation with the U.S. Environmental Protection Agency to provide systematic, long-term monitoring to better quantify the seasonal and annual variability in selected constituents.

The purpose of this report is to present water-quality data for 18 sites and trace-element data for 13 bed-sediment and biological sites in the upper Clark Fork basin collected from October 2004 through September 2005 (water year 2005). Quality-assurance data are presented for water-quality, bedsediment, and biota samples. Statistical summaries also are provided for water-quality, bed-sediment, and biological data collected since 1985.

\section{Sampling Locations and Types of Data}

Sampling sites in the upper Clark Fork basin from Butte to below Missoula (fig. 1) are located on the Clark Fork mainstem, six major tributaries (Blacktail Creek, Warm Springs Creek, Little Blackfoot River, Flint Creek, Rock Creek, and Blackfoot River), and three smaller tributaries (Mill Creek, Willow Creek, and Lost Creek). The sites, types of data collected, and period of record for each data type are listed in table 1. Mainstem sampling sites were selected to divide the upper Clark Fork into reaches of relatively uniform length, with each reach encompassing either a major tributary or depositional environment (Warm Springs Ponds

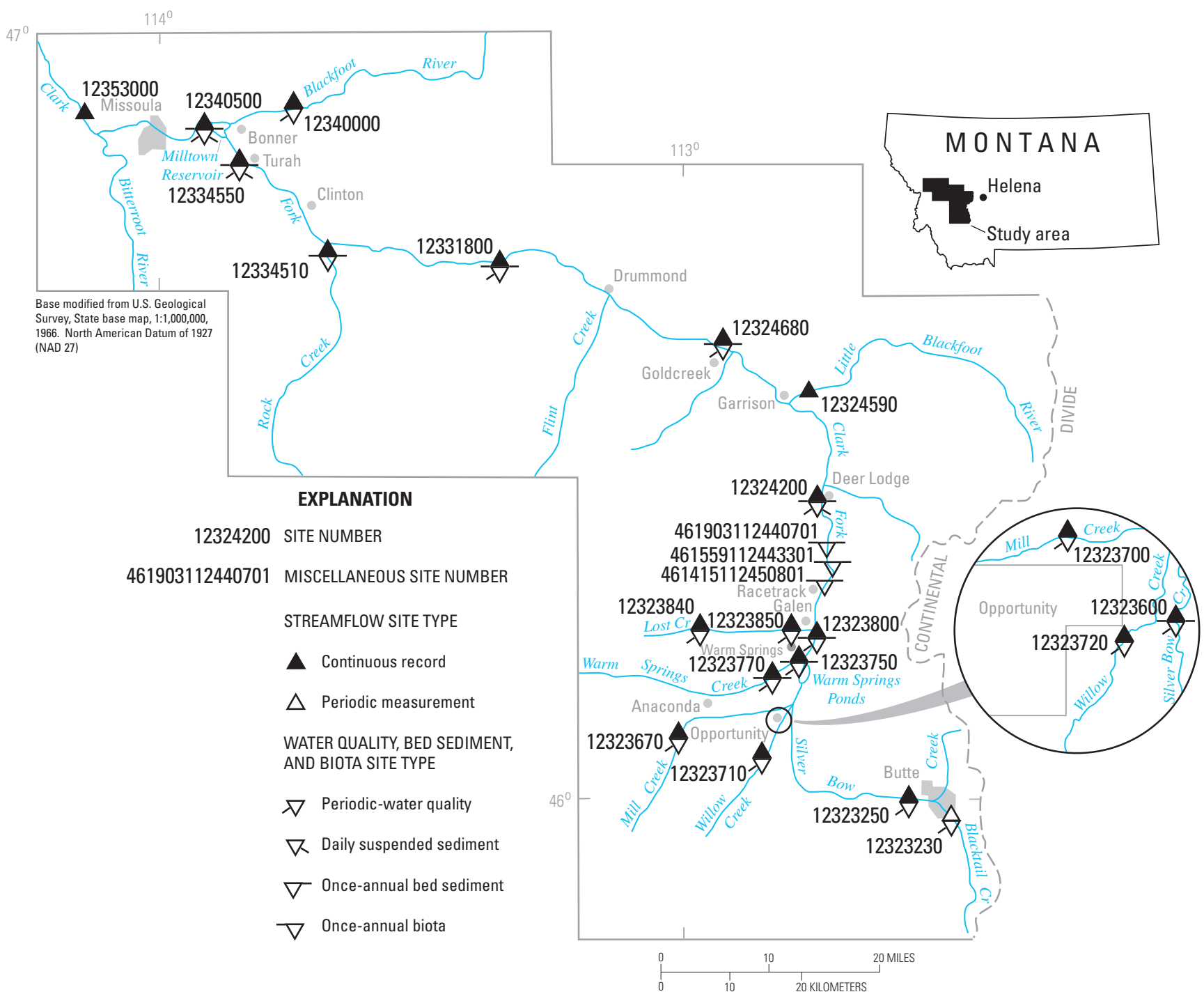

Figure 1. Location of study area. 
and Milltown Reservoir). Major tributaries were sampled to describe water-quality, bed-sediment, and biological characteristics of important hydrologic sources in the upper basin and to provide reference comparisons to the mainstem. The three smaller tributaries were sampled to gain better spatial resolution on sources of metals entering the Clark Fork in an area of historical metal-processing activities near Anaconda. Water-quality data were obtained periodically at 18 sites; daily suspended-sediment data were obtained at 3 of these sites. Trace-element data for 13 bed-sediment and 13 biological sites were obtained once-annually. Continuous streamflow data were collected at 20 sites.

A list of properties measured onsite and constituents analyzed in samples of water, bed sediment, and biota is given in table 2. Data-quality objectives for analyses of water-quality samples are listed in table 3. Onsite measurements of properties, laboratory analyses of water, bed-sediment, and biota samples, and associated quality-assurance data for water year 2005 are listed in tables 4 through 19 at the back of the report. Statistical summaries of water-quality, bed-sediment, and biological data collected between March 1985 and September 2005 are given in tables 20 through 22 at the back of the report.

Quality assurance of data was maintained through the use of documented procedures designed to provide environmentally representative data. Acceptable performance of the procedures was verified with quality-control samples that were collected systematically to provide a measure of the accuracy, precision, and bias of the environmental data and to identify problems associated with sampling, processing, or analysis.

\section{Water-Quality Data}

Water-quality data consist of onsite measurements of selected stream properties and concentrations of chemical and physical constituents analyzed in periodically collected stream samples. At 17 sites on the Clark Fork mainstem and tributaries, samples were collected 8 times per year on a schedule designed to describe seasonal and hydrologic variability. At Warm Springs Creek at Warm Springs, samples were collected 6 times during the water year. At the three daily suspendedsediment sites, suspended-sediment samples were collected 2 to 9 times per week during water year 2005 (table 1).

\section{Methods}

Water samples were collected from vertical transits throughout the entire stream depth at multiple locations across the stream using depth- and width-integration methods described by Ward and Harr (1990), Edwards and Glysson (1999), and the USGS National Field Manual for the Collection of Water-Quality Data (variously dated). These methods provide a vertically and laterally discharge-weighted compos- ite sample that is intended to be representative of the entire flow passing through the cross section of a stream. Sampling equipment consisted of depth-integrating suspended-sediment samplers (DH-48, DH-81, and D-74TM), which were either constructed of plastic or coated with a non-metallic epoxy paint, and equipped with nylon or Teflon nozzles.

Instantaneous streamflow at the time of water sampling was determined at all sites, either by direct measurement or from stage-discharge rating tables (Rantz and others, 1982). Onsite measurements of specific conductance, $\mathrm{pH}$, and water temperature were made during collection of periodic waterquality samples. Onsite sample processing, including filtration and preservation, was performed according to procedures described by Ward and Harr (1990), Horowitz and others (1994), and USGS (variously dated).

Water samples were analyzed for the constituents listed in table 2. The terms "filtered" and "unfiltered recoverable" replace the terms "dissolved" and "total recoverable," respectively, which were used in the past. The trace elements (arsenic, cadmium, copper, iron, lead, manganese, and zinc) were analyzed for filtered $(0.45-\mu \mathrm{m}$ pore size $)$ and unfiltered recoverable concentrations by the USGS National Water Quality Laboratory (NWQL) in Denver, Colo. Concentrations of calcium and magnesium also were determined in filtered samples to enable calculation of hardness. Filtered concentrations of arsenic, cadmium, copper, lead, manganese, and zinc were analyzed by inductively coupled plasma-mass spectrometry (Faires, 1993; Garbarino, 1999). Filtered concentrations of calcium, magnesium, and iron were analyzed by inductively coupled plasma-atomic emission spectrometry (Fishman, 1993). Unfiltered recoverable concentrations of trace elements were determined from unfiltered samples that were first digested with dilute hydrochloric acid (Hoffman and others, 1996) and then analyzed by inductively coupled plasma-mass spectrometry (Garbarino and Struzeski, 1998). For part of water year 2005, unfiltered recoverable arsenic was analyzed by graphite furnace-atomic absorption spectrometry (Jones and Garbarino, 1999).

Water samples also were collected from multiple vertical transits for analysis of suspended sediment whenever periodic water-quality samples were collected. These samples were analyzed for suspended-sediment concentration and the percentage of suspended-sediment mass finer than 0.062-mm diameter (silt size and smaller) by the USGS Montana Water Science Center sediment laboratory (herein referred to as Montana Sediment Laboratory) in Helena, Mont., according to methods described by Guy (1969) and Dodge and Lambing (2006).

Suspended-sediment samples for the three daily suspended sediment sites were collected by local contract observers using the depth-integration method at a single vertical near mid-stream. The samples were analyzed for suspended-sediment concentration and were used to determine daily mean suspended-sediment concentrations according to methods described by Porterfield (1972). 


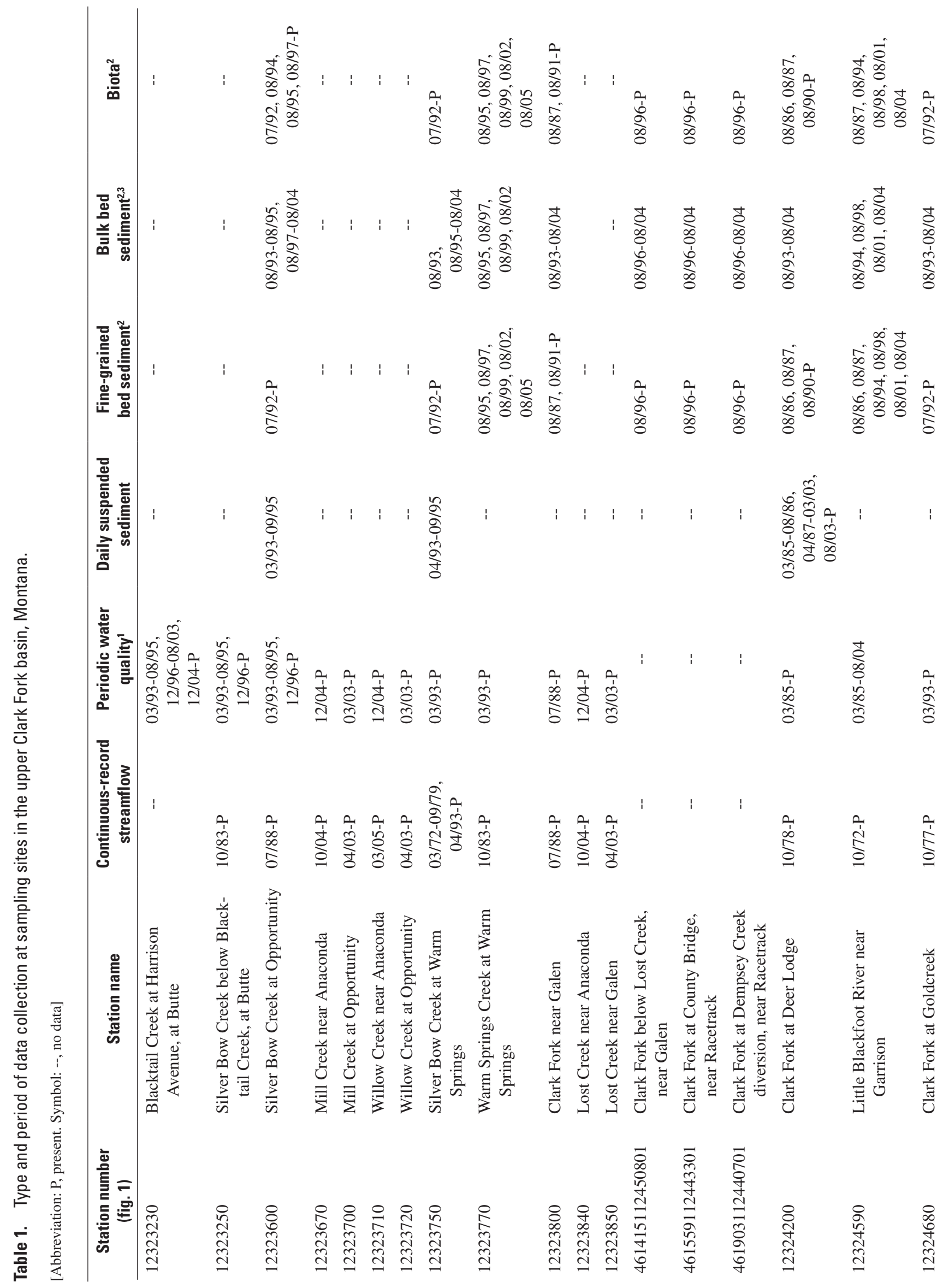




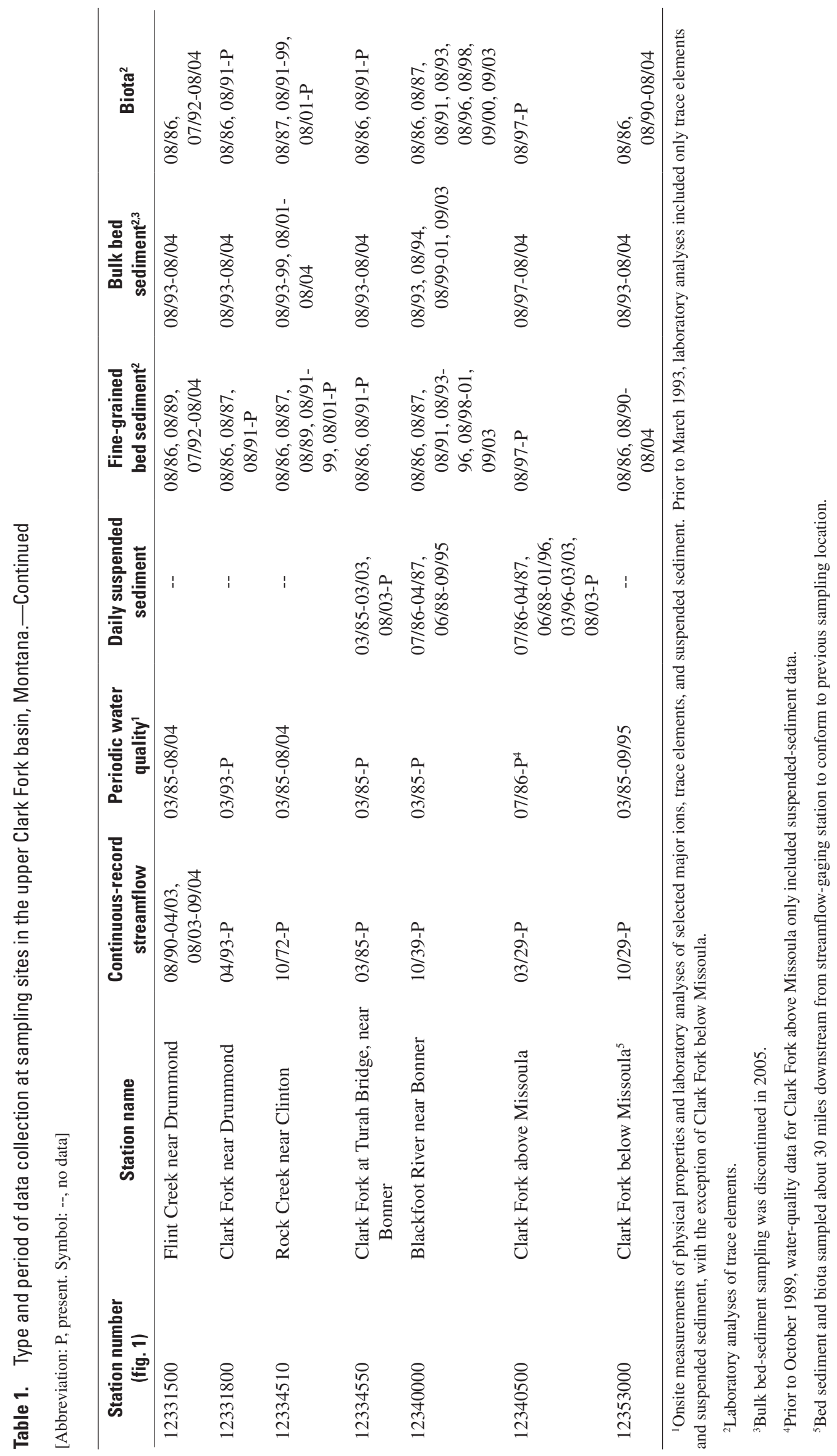


Table 2. Properties measured onsite and constituents analyzed in samples of water, bed sediment, and biota from the upper Clark Fork basin, Montana.

\begin{tabular}{|c|c|c|c|}
\hline \multicolumn{2}{|c|}{ Water } & \multirow{2}{*}{$\begin{array}{c}\text { Bed Sediment } \\
\text { Constituent }\end{array}$} & \multirow{2}{*}{$\begin{array}{c}\text { Biota } \\
\text { Constituent }\end{array}$} \\
\hline Property & Constituent & & \\
\hline Streamflow & Hardness (calculated) & Arsenic & Arsenic \\
\hline Specific conductance & Calcium & Cadmium & Cadmium \\
\hline $\mathrm{pH}$ & Magnesium & Chromium & Chromium \\
\hline \multirow[t]{8}{*}{ Temperature } & Arsenic & Copper & Copper \\
\hline & Cadmium & Iron & Iron \\
\hline & Copper & Lead & Lead \\
\hline & Iron & Manganese & Manganese \\
\hline & Lead & Nickel & Nickel \\
\hline & Manganese & Zinc & Zinc \\
\hline & Zinc & & \\
\hline & Suspended sediment & & \\
\hline
\end{tabular}

Table 3. Data-quality objectives for analyses of water-quality samples collected in the upper Clark Fork basin, Montana.

[Abbreviations: $\mu \mathrm{g} / \mathrm{L}$, micrograms per liter; mg/L, milligrams per liter; mm, millimeter. Symbol: --, not determined]

\begin{tabular}{|c|c|c|c|}
\hline \multirow[b]{3}{*}{ Constituent } & \multicolumn{3}{|c|}{ Data-quality objectives } \\
\hline & Detectability & Precision & Bias \\
\hline & $\begin{array}{c}\text { Laboratory reporting } \\
\text { level }\end{array}$ & $\begin{array}{l}\text { Maximum relative standard } \\
\text { deviation of laboratory } \\
\text { replicate analyses, } \\
\text { in percent } \\
\end{array}$ & $\begin{array}{l}\text { Maximum deviation } \\
\text { of spike recovery, } \\
\text { in percent }\end{array}$ \\
\hline Calcium, filtered & $0.02 \mathrm{mg} / \mathrm{L}$ & 20 & -- \\
\hline Arsenic, filtered & $.2 \mu \mathrm{g} / \mathrm{L}$ & 20 & 25 \\
\hline Arsenic, unfiltered recoverable ${ }^{1}$ & $.12-2 \mu \mathrm{g} / \mathrm{L}$ & 20 & 25 \\
\hline Cadmium, filtered & $.04 \mu \mathrm{g} / \mathrm{L}$ & 20 & 25 \\
\hline Cadmium, unfiltered recoverable & $.04 \mu \mathrm{g} / \mathrm{L}$ & 20 & 25 \\
\hline Iron, unfiltered recoverable & $6 \mu \mathrm{g} / \mathrm{L}$ & 20 & 25 \\
\hline Lead, filtered & $.08 \mu \mathrm{g} / \mathrm{L}$ & 20 & 25 \\
\hline Lead, unfiltered recoverable & $.06 \mu \mathrm{g} / \mathrm{L}$ & 20 & 25 \\
\hline Manganese, filtered & $.2 \mu \mathrm{g} / \mathrm{L}$ & 20 & 25 \\
\hline Manganese, unfiltered recoverable & $.2 \mu \mathrm{g} / \mathrm{L}$ & 20 & 25 \\
\hline Zinc, filtered & $.6 \mu \mathrm{g} / \mathrm{L}$ & 20 & 25 \\
\hline Zinc, unfiltered recoverable & $2 \mu \mathrm{g} / \mathrm{L}$ & 20 & 25 \\
\hline Sediment, suspended, percent finer than $0.062 \mathrm{~mm}$ & 1 percent & -- & -- \\
\hline
\end{tabular}

\footnotetext{
${ }^{1}$ The laboratory reporting level changed during water year 2005 .
} 


\section{Results}

Water-quality data for samples collected periodically during water year 2005 are presented in table 4. Daily mean streamflow, daily mean suspended-sediment concentration, and daily suspended-sediment discharge for water year 2005 at the three daily suspended-sediment stations are reported in tables 5 through 7 , along with monthly summary statistics and annual totals for streamflow and sediment load.

\section{Quality Assurance}

Quality-assurance procedures used for the collection and field processing of water-quality samples are described by Ward and Harr (1990), Knapton and Nimick (1991), Horowitz and others (1994), Edwards and Glysson (1999), and USGS (variously dated). Standard procedures used by the NWQL for internal sample handling and quality assurance are described by Friedman and Erdmann (1982), Jones (1987), and Pritt and Raese (1995). Quality-assurance procedures used by the Montana Sediment Laboratory are described by Dodge and Lambing (2006).

The quality of analytical results reported for waterquality samples was evaluated by quality-control samples that were submitted from the field and analyzed concurrently in the laboratory with routine samples. These quality-control samples consisted of replicates, spikes, and blanks which provide quantitative information on the precision and bias of the overall field and laboratory process. Each type of qualitycontrol sample was submitted at a proportion equivalent to about 5 percent of the total number of water-quality samples. Therefore, the total number of quality-control samples represented about 15 percent of the total number of water-quality samples.

In addition to quality-control samples submitted from the field, internal quality-assurance practices at the NWQL are performed systematically to provide quality control of analytical procedures (Pritt and Raese, 1995). These internal practices include analyses of quality-control samples such as calibration standards, standard reference water samples, replicate samples, deionized-water blanks, or spiked samples at a proportion equivalent to at least 10 percent of the sample load. The NWQL participates in a blind-sample program where standard reference water samples prepared by the USGS Branch of Quality Systems are routinely inserted into the sample line for each analytical method at a frequency proportional to the sample load. The laboratory also participates in external evaluation studies and audits with the National Environmental Laboratory Accreditation Program, U.S. Environmental Protection Agency, Environment Canada, and the USGS Branch of Quality Systems to assess analytical performance.

Replicate data can be obtained in different ways to provide an assessment of precision (reproducibility) of analytical results. Replicate samples are two or more samples considered to be essentially identical in composition. Replicate samples can be obtained in the field (field replicate) by either repeating the collection process to obtain two or more independent composite samples, or by splitting a single composite sample into two or more subsamples. The individual replicate samples are then analyzed separately. Likewise, a single sample can be analyzed two or more times in the laboratory to obtain a measure of analytical variability (laboratory replicate).

Precision of analytical results for field replicates is affected by numerous sources of variability within the field and laboratory environments, including sample collection, sample processing, and sample analysis. To provide data on precision for samples exposed to all sources of variability, replicate stream samples for chemical analysis were obtained in the field by splitting a composite stream sample, and replicate stream samples for suspended-sediment analysis were obtained in the field by concurrently collecting two independent cross-sectional samples. Analyses of these field replicates indicate the reproducibility of environmental data that are affected by the combined variability potentially introduced by field and laboratory processes.

Analytical precision was evaluated with laboratory replicates, which exclude field sources of variability. Two independent analyses were made of an individual sample selected randomly in the laboratory from the group of samples comprising each analytical run. A separate analysis of the sample was made at the beginning and end of each analytical run to provide information on the reproducibility of laboratory analytical results independent of possible variability caused by field collection and processing of samples.

Spiked samples are used to evaluate bias, which measures the ability of an analytical method to accurately quantify a known amount of analyte added to a sample. Because some constituents in stream water can potentially interfere with the analysis of a targeted analyte, it is important to determine whether such effects are causing biased (consistently high or low) results. Deionized-water blanks and aliquots of stream samples were spiked in the laboratory with known amounts of the same trace elements analyzed in water samples. Analyses of spiked blanks indicate if the spiking procedure and analytical method are within control for a water matrix that is presumably free of chemical interference. Analyses of spiked aliquots of stream samples indicate if the chemical matrix of the stream water interferes with the analytical measurement and whether these interferences could contribute significant bias to reported trace-element concentrations for stream samples.

Blank samples of deionized water were submitted for every field trip and analyzed to identify the presence and magnitude of contamination that potentially could bias analytical results. The particular type of blank sample routinely tested was a "field" blank. Field blanks are aliquots of deionized water that are certified as trace-element free and are processed through the sampling equipment used to collect stream samples. These blanks are then subjected to the same processing (sample splitting, filtration, preservation, transportation, and laboratory handling) as stream samples. Blank samples are 
analyzed for the same constituents as those of stream samples to identify whether any detectable concentrations exist.

All water samples were handled in accordance with chain-of-custody procedures that provide documentation of sample identity, shipment, receipt, and laboratory handling. All routine and quality-control samples submitted from a sampling episode were stored in a secure area of the NWQL and analyzed as a discrete sample group, independent of other samples submitted to the NWQL. Therefore, the qualitycontrol data apply solely to the analytical results for stream samples reported herein and provide a direct measure of data quality for this monitoring program.

Data-quality objectives (table 3) were established for water-quality data as part of the study plan for the expanded long-term monitoring program that was initiated in 1993. The objectives identify analytical requirements of detectability and serve as a guide for identifying questionable data by establishing acceptable limits for precision and bias of laboratory results. Comparisons of quality-control data to data-quality objectives are used to evaluate whether sampling and analytical procedures are producing environmentally representative data in a consistent manner. Data that did not meet the objectives were evaluated for acceptability. If necessary, additional quality-control samples were submitted and corrective action was taken.

The NWQL uses a statistically based convention for establishing minimum laboratory reporting levels for analytical results and for reporting low-concentration data (Childress and others, 1999). Quality-control data are collected by the NWQL on a continuing basis to determine long-term method detection levels (LT-MDLs) and laboratory reporting levels (LRLs). These values are reevaluated each year and, consequently, can change from year to year. The methods are designed to limit the possible occurrence of a false positive or false negative error to 1 percent or less. Accordingly, concentrations are reported as less than the LRL for samples in which the analyte was either not detected or did not pass identification criteria. Analytes that are detected at concentrations between the LT-MDL and LRL and that pass identification criteria are estimated. Estimated concentrations are noted with a remark code of "E." These data need to be used with the understanding that their uncertainty is greater than that of data reported without the "E" remark code.

The precision of analytical results for a constituent can be determined by estimating a standard deviation of the differences between replicate measurements for several sets of samples. These replicate measurements may consist either of individual analyses of a pair of samples considered to be essentially identical (field replicates) or multiple analyses of an individual sample (laboratory replicates). The differences in concentration between replicate analyses can be used to estimate a standard deviation according to the following equation (Taylor, 1987):

$$
S=\sqrt{\frac{\sum d^{2}}{2 k}}
$$

where

$S \quad$ is the standard deviation of the difference in concentration between replicate analyses,

$d \quad$ is the difference in concentration between each pair of replicate analyses,

and

$k \quad$ is the number of pairs of replicate analyses.

Precision also can be expressed as a relative standard deviation (RSD), in percent, which is computed from the standard deviation and the mean concentration for all the replicate analyses. Expressing precision relative to a mean concentration standardizes comparison of precision among individual constituents. The RSD, in percent, is calculated according to the following equation (Taylor, 1987):

$$
R S D=\frac{S}{\bar{x}} \times 100
$$

where

$$
\begin{aligned}
R S D & \text { is the relative standard deviation, } \\
& \text { is the standard deviation, }
\end{aligned} \text { and } \quad \begin{aligned}
& \text { is the mean of all replicate concentrations. }
\end{aligned}
$$

Paired analyses of field replicates are presented in table 8 . The precision estimated for each constituent based on these paired results, which include both field and laboratory sources of variability, is reported in table 9. Data-quality objectives for precision are not directly applicable to field replicates because of the inability to determine whether the variability results from field sample collection and processing, or laboratory handling and analysis. However, the precision for field replicate analyses is calculated to illustrate overall reproducibility of environmental data that incorporates both field and laboratory sources of variability. The data-quality objective used to indicate acceptable precision of results for field replicates was a maximum relative standard deviation of 20 percent (table 3 ). Precision estimates for field replicate analyses were within the 20-percent relative standard deviation limit for all constituents (table 9).

Analytical precision for chemical constituents based on laboratory replicate analyses of individual samples, which includes only laboratory sources of variability, is reported in table 10. Statistics for analytical precision of laboratoryreplicate analyses are based on unrounded values stored in laboratory data files. The data-quality objective for analytical precision of laboratory-replicate analyses was a maximum relative standard deviation of 20 percent (table 3). Precision estimates for laboratory-replicate analyses were less than the 20-percent relative standard deviation limit for all constituents (table 10); thus, the data-quality objectives for precision were met.

Recovery efficiency for analyses of constituents is determined by analyses of an unspiked sample and a spiked 
aliquot of the same sample. The data-quality objective for acceptable spike recovery of trace elements in water samples was a maximum deviation of 25 percent from a theoretical 100-percent recovery of added constituent (table 3 ). At the laboratory, a spiked deionized-water blank and a spiked aliquot of a stream sample were prepared and analyzed along with the original unspiked sample. The differences between the spiked and unspiked sample concentrations were determined and used to compute recovery, in percent, according to equation 3:

$$
R=\frac{D}{C} \times 100
$$

where

$R \quad$ is the spike recovery, in percent;

$D \quad$ is the difference between the spiked sample concentration and unspiked sample concentration;

and

$$
\begin{aligned}
& C \quad \text { is the concentration of material used to spike } \\
& \text { the sample. }
\end{aligned}
$$

If the spike recovery for a trace element was outside a range of 75 to 125 percent, the instrument was recalibrated and the entire sample set and spiked samples were reanalyzed for that particular trace element until recoveries were improved to the extent possible. Results of recovery efficiency for individual trace elements in spiked deionized-water blanks and spiked stream samples are presented in tables 11 and 12 , respectively. The mean spike recovery for deionized-water samples spiked with trace elements (table 11) ranged from 93.8 to 104 percent. The 95 -percent confidence intervals (Taylor, 1987) for the mean spike recovery for each constituent analyzed in deionized-water samples (table 11) did not exceed a 25-percent deviation from an expected 100-percent recovery. The mean spike recovery for spiked stream samples (table 12) ranged from 88.3 to 104 percent. The 95-percent confidence intervals for the mean of spike recovery for each constituent analyzed in stream samples (table 12) did not exceed a 25-percent deviation from an expected 100-percent recovery, except for filtered cadmium (62.7-133), filtered copper (57.9-129), and filtered zinc (60.8-128). No adjustments were made to analytical data for filtered cadmium, copper, and zinc because the mean spike recoveries were 97.8, 93.6, and 94.3 percent, respectively, which indicated that there was no systematic bias.

High or low bias is indicated if the confidence interval does not include 100 percent recovery, thereby indicating a consistent deviation in one direction. All laboratory-spiked deionized-water blank samples (table 11) had confidence intervals for percent recovery that included 100 percent, except filtered arsenic (95.5-99.3 percent). The 95-percent confidence intervals for percent recovery in all laboratory-spiked stream samples (table 12) included 100 percent, except unfiltered recoverable arsenic (101-108) and unfiltered recoverable manganese (78.3-98.3). Both the 95-percent confidence interval (101-108) and mean (104) percent recoveries for unfiltered recoverable arsenic in laboratory-spiked stream samples indicate a persistent, but very minor, high bias. In contrast, the 95-percent confidence interval (90.7-111) and mean (101) percent recoveries for unfiltered recoverable arsenic in laboratory-spiked deionized water blanks (table 11) indicate no bias. Thus, it appears that the minor bias in spiked stream samples is not necessarily caused by the analytical method, but might be a result of analytical interference from the chemical matrix of the stream samples. The same comparison is made for laboratory spiked stream samples for unfiltered recoverable manganese. Both the 95-percent confidence interval (78.3-98.3) and mean (88.3) percent recoveries for unfiltered recoverable manganese in laboratory-spiked stream samples (table 12) indicate a persistent low bias of moderate magnitude. However, the 95-percent confidence interval (82.2-105) and mean (93.8) percent recoveries for laboratoryspiked deionized water (table 11) indicate no bias. Because the mean spike recoveries for all constituents met data-quality objectives, no adjustments were made to analytical results for stream samples on the basis of spike recoveries.

Analytical results for field blanks are presented in table 13. A field blank with constituent concentrations equal to or less than the LRL for the analytical method indicates that the entire process of sample collection, field processing, and laboratory analysis is presumably free of contamination. If detectable concentrations in field blanks were equal to or greater than twice the LRL, the concentrations were noted during data review. Analytical results from the field blank for the next sample set were evaluated for a consistent trend that may indicate systematic contamination. Sporadic, infrequent exceedances of twice the LRL probably represent random contamination or instrument calibration error that is not persistent in the process and which is not likely to cause positive bias in a long-term record of analytical results. However, if concentrations for a particular constituent exceeded twice the LRL in field blanks from two consecutive field trips, blank samples were collected from individual components of the processing sequence and were submitted for analysis in order to identify the source of contamination.

Trace-element concentrations in field blanks (table 13) were almost always less than the LRL. Two detections exceeded twice the LRL in two separate samples. One occurred on March 7 for filtered zinc $(1.9 \mu \mathrm{g} / \mathrm{L})$ which was greater than the LRL of $0.6 \mu \mathrm{g} / \mathrm{L}$; several other blanks exceeded the LRL for filtered zinc but they did not exceed twice the LRL. The other occurred on June 2 for unfiltered recoverable iron $(30 \mu \mathrm{g} / \mathrm{L})$ which was greater than the LRL of $6 \mu \mathrm{g} / \mathrm{L}$. Since no trends were indicated in subsequent sampling trips, no adjustments were made to water-quality sample results based on these two detections. 


\section{Bed-Sediment Data}

Bed-sediment data consist of analyses of trace-element concentrations in the fine-grained fraction of bed-sediment samples. Collection of bulk bed sediment (fine-grained plus coarse-grained fractions) was discontinued in 2005; therefore, no analytical results or statistical summaries are presented. Bed-sediment samples were collected once-annually during low, stable flow conditions and the same season (typically August) as previous samples to facilitate data comparisons among years.

\section{Methods}

Fine-grained bed-sediment samples were collected in August 2005 using protocols described by E.V. Axtmann (U.S. Geological Survey, written commun., 1994). Samples were collected from the surfaces of streambed deposits in low-velocity areas near the edge of the stream using an acidwashed polypropylene scoop. Whenever possible, samples were collected from both sides of the stream. Three composite samples of bed sediment were collected at each site.

Individual samples of bed sediment were collected by scooping material from the surfaces of three to five randomly selected deposits along pool or low-velocity areas. The three to five individual samples were combined to form a single composite sample. This collection process was repeated three times to obtain three composite samples. Each composite sample was wet-sieved onsite through a 0.064-mm nylon-mesh sieve using ambient stream water. The fraction of bed sediment in each composite sample that was finer than $0.064 \mathrm{~mm}$ was transferred to an acid-washed 500-mL polyethylene bottle and transported to the laboratory on ice.

Bed-sediment samples were processed and analyzed at the USGS National Research Program Ecology and Contaminants Project laboratory in Menlo Park, Calif. Bed-sediment samples were oven-dried at $60^{\circ} \mathrm{C}$ and ground using an acidwashed ceramic mortar and pestle. Single aliquots of approximately $0.6 \mathrm{~g}$ of sediment from each of the three composite bed-sediment samples were digested using a hot, concentrated, nitric acid reflux according to methods described by Luoma and Bryan (1981). An additional aliquot was analyzed from one of the sieved replicate samples at each station. After a digestion period of up to 2 weeks, the aliquots were evaporated to dryness on a hot plate. The dry residue was reconstituted in $10 \mathrm{~mL}$ of $0.6 \mathrm{~N}$ (normal) hydrochloric acid. The reconstituted aliquots then were filtered through a $0.45-\mu \mathrm{m}$ filter using a syringe and in-line disposable filter cartridge. The filtrate was diluted to a 1:10 ratio with $0.6 \mathrm{~N}$ hydrochloric acid. These final solutions were analyzed for arsenic, cadmium, chromium, copper, iron, lead, manganese, nickel, and zinc using inductively coupled argon plasma-emission spectroscopy (ICAPES). The smallest concentration of a constituent that can be reliably reported for analyses of bed sediment is termed the minimum reporting level.

\section{Results}

Concentrations of trace elements measured in samples of fine-grained bed sediment collected during August 2005 are summarized in table 14. Liquid-phase concentrations, in $\mu \mathrm{g} / \mathrm{mL}$, that were analyzed in the reconstituted aliquots of digested bed sediment were converted to solid-phase concentrations, in $\mu \mathrm{g} / \mathrm{g}$, using the following equation:

$$
\mu \mathrm{g} / \mathrm{g}=\frac{\mu \mathrm{g} / \mathrm{mL} \times \text { volume of digested sample, in } \mathrm{mL}}{\text { dry weight of sample, in } \mathrm{g} \times \text { dilution ratio }}
$$

The reported solid-phase concentrations in table 14 are the mean of all analyses for replicate aliquots from each composite bed-sediment sample collected at the site. Because the conversion from liquid-phase to solid-phase concentration is dependent on both the dilution ratio and the dry weight of the sample, minimum reporting levels for some trace elements might differ among stations and among years.

\section{Quality Assurance}

The protocols for field collection and processing of bedsediment samples are designed to prevent contamination from metal sources. Non-metallic sampling and processing equipment was acid-washed and rinsed with deionized water prior to the first sample collection. Nylon-mesh sieves were washed in a laboratory-grade detergent and rinsed with deionized water. All equipment received a final rinse onsite with stream water. Sampling equipment used at more than one site was rinsed between sites with stream water. Separate sieves were used at each site and, therefore, did not require between-site cleaning. Bed-sediment samples were collected sequentially at sites along an increasing concentration gradient to minimize effects from potential site-to-site carryover contamination.

Quality assurance of analytical results for bed-sediment samples included laboratory instrument calibration with standard solutions and analysis of quality-control samples designed to identify the presence and magnitude of bias (E.V. Axtmann, U.S. Geological Survey, written commun., 1994). Quality-control samples consisted of standard reference materials and procedural blanks. Each type of qualitycontrol sample was analyzed in a proportion equivalent to about 10 percent of the total number of bed-sediment samples.

Standard reference materials (SRMs) are commercially prepared materials that have certified concentrations of trace elements. Analyses of SRMs are used to indicate the ability of the method to accurately measure a known quantity of a constituent. Multiple analyses of the SRMs are made to derive a mean and 95-percent confidence interval for recovery. Recovery efficiency for trace-element analyses of SRMs for bed sediment is summarized in table 15. Two SRMs consisting of agricultural soils representing low and high concentrations of trace elements were analyzed to test recovery efficiency for a range of concentrations generally similar to those occurring 
in the bed sediment of streams in the upper Clark Fork basin. The digestion process used to analyze bed-sediment samples is not a "total" digestion (does not liberate elements associated with crystalline lattices); therefore, 100-percent recovery may not be achieved for elements strongly bound to the sediment. The percent recovery of trace elements for SRM analyses that use less than a total digestion is useful to indicate which trace elements display strong sediment-binding characteristics in the SRM and whether analytical recovery is consistent between multiple sets of analyses.

Although data-quality objectives have not been established for bed sediment, percent recoveries for individual trace elements shown in table 15 illustrate analytical performance. Mean SRM recoveries for the low-concentration standard (SRM 2709) ranged from 82.2 to 89.1 percent of the certified concentrations for copper, iron, lead, manganese, and nickel. Mean recoveries were lower for arsenic, chromium, and zinc (65.4, 60.2, and 75.6 percent, respectively). Cadmium concentrations were near the minimum reporting level in SRM 2709 and were not reported. The lack of measurable recoveries for cadmium in the SRM is likely the result of analyzing concentrations very close to the liquid-phase detection limit $(0.0003 \mu \mathrm{g} / \mathrm{mL})$ coupled with signal enhancement resulting from matrix interference. Percent recoveries for arsenic, cadmium, copper, iron, lead, manganese, and zinc in the high-concentration standard (SRM 2711) ranged from 80.6 to 94.4 percent of the certified concentration. Chromium and nickel had lower recoveries (51.5 and 78.9 percent, respectively) for the high-concentration SRM, possibly due to the strong binding nature of these elements to sediment. The generally small range of variation (less than 10 percent for most constituents) for the 95-percent confidence interval indicates good reproducibility of multiple analyses of the SRMs. No adjustments were made to trace-element concentrations in bed-sediment samples on the basis of recovery efficiencies.

Procedural blanks for bed-sediment samples consisted of the same reagents used for sample digestion and reconstitution. Concentrated nitric acid used for sample digestion was heated and evaporated to dryness. After evaporation, $0.6 \mathrm{~N}$ hydrochloric acid was added to reconstitute the dry residue. Procedural blanks, therefore, represent the same chemical matrix and exposure to analytical materials and handling as the reagents used to digest and reconstitute bed-sediment samples. Analytical results of procedural blanks for bed sediment (table 16) are reported as a liquid-phase concentration, in $\mu \mathrm{g} / \mathrm{mL}$, which is equivalent to parts per million. A procedural blank was prepared and analyzed concurrently with bed-sediment samples for each site. Concentrations of trace elements in all procedural blanks were below the minimum reporting level; thus, no contamination bias was indicated and no adjustments to the data were necessary.

\section{Biological Data}

Biological data consist of analyses of trace-element concentrations in the whole-body tissue of aquatic benthic insects. Insect samples were collected once-annually at the same sites and dates as bed-sediment samples (table 1), allowing for a direct comparison of biological data with bedsediment data among the years.

\section{Methods}

Insect samples were collected using protocols described in Hornberger and others (1997). Immature stages of benthic insects were collected using a large nylon-mesh kick net. A single riffle at each station was sampled repeatedly until an adequate number of individual insects were collected to provide sufficient mass for analysis. Targeted taxa for collection were the Order Trichoptera (caddisflies) and the Order Plecoptera (stoneflies).

Two caddisfly species of the genus Hydropsyche (Hydropsyche cockerelli and Hydropsyche occidentalis) were targeted for collection in this study due to their occurrence at most, but not all, sites. In a few instances, Hydropsyche tana were collected. Hydropsyche species (spp.) that could not be positively identified were considered to belong to the morosa group and are categorized as Hydropsyche spp. or Hydropsyche morosa group (in previous reports). The caddisfly Arctopsyche grandis and the stonefly Claasenia sabulosa were collected where available to represent additional insect taxa that are commonly distributed in the upper Clark Fork basin. In addition, the caddisfly group Brachycentrus spp. was sometimes collected when targeted taxa were not available.

Samples of each taxon were sorted by genus in the field and placed in acid-washed plastic containers. Samples were frozen on dry ice within 30 minutes of collection in a small amount of ambient stream water. Between 1986 and 1998, macroinvertebrate containers were kept on ice to allow the insects to evacuate their gut contents for a period of 6 to 8 hours. Excess water was drained and insects were frozen for transport to the laboratory. In order to reduce the possibility of metal loss through intracellular breakdown during depuration, samples were frozen on dry ice in the field between 1999 and 2005. A comparison of immediately frozen versus depurated samples showed that while no significant difference occurred for most metals, concentrations of copper in the depurated macroinvertebrate samples were about 20 percent lower than those which were immediately frozen. The data were not adjusted for this difference.

Insect samples were processed and analyzed at the USGS National Research Program, Ecology and Contaminants Project laboratory in Menlo Park, Calif. Insects were thawed 
and rinsed with ultra-pure deionized water to remove particulate matter and then sorted to their lowest possible taxonomic level. If large numbers of specimens had been collected at a site, similar-sized individuals were composited into replicate subsamples. Subsamples were placed in tared scintillation vials and oven-dried at $70^{\circ} \mathrm{C}$. Subsamples were weighed to obtain a final dry weight and digested by reflux using concentrated nitric acid (Cain and others, 1992). After digestion, insect samples were evaporated to dryness on a hot plate. The dry residue was reconstituted in $0.6 \mathrm{~N}$ hydrochloric acid, filtered through a $0.45-\mu \mathrm{m}$ filter, and analyzed undiluted by ICAPES for arsenic, cadmium, chromium, copper, iron, lead, manganese, nickel, and zinc. The smallest concentration of a constituent that can be reliably reported for analyses of biota is termed the minimum reporting level.

\section{Results}

Concentrations of trace elements in whole-body tissue of aquatic insects collected during August 2005 are summarized in table 17 . The variability in the number of composite samples among species and among sites reflects differences in insect abundance, with the number of composite samples increasing with the relative abundance of insects. Liquidphase concentrations, in $\mu \mathrm{g} / \mathrm{mL}$, analyzed in the reconstituted samples were converted to solid-phase concentrations, in $\mu \mathrm{g} / \mathrm{g}$, using equation 4 . As with bed sediment, minimum reporting levels may differ among sites as a result of variable sample weights. In general, the smaller the biological sample weight (a function of insect abundance), the higher the minimum reporting level. Therefore, higher minimum reporting levels do not necessarily imply a higher trace-element concentration in tissue.

\section{Quality Assurance}

The protocols for field collection and processing of biota samples are designed to prevent contamination from metal sources. Non-metallic nets, sampling equipment, and processing equipment were employed in all sample collection. Equipment was acid-washed and rinsed in ultra-pure deionized water prior to the first sample collection. Nets and equipment were thoroughly rinsed in ambient stream water at each new mainstem site. New nets were used for all tributary sites. Biota samples were collected sequentially at sites along an increasing concentration gradient to minimize effects from potential site-to-site carryover contamination.

Quality assurance of analytical results for biota samples included laboratory instrument calibration with standard solutions and analyses of quality-control samples designed to quantify precision and to identify the presence and magnitude of bias. Quality-control samples consisted of 12 replicates of the tissue SRM (lobster hepatopancreas) and procedural blanks for each station. Quality-control samples were analyzed in a proportion equivalent to about 20 percent of the total number of biota samples.

Recovery efficiency for trace-element analyses of the SRM for biota is summarized in table 18. The reference material tested was lobster hepatopancreas. Data-quality objectives have not been established for analytical recovery in biota, but percent recoveries are shown to illustrate analytical performance. Mean SRM recoveries ranged from 92.6 to 100 percent of the certified concentrations for arsenic, cadmium, copper, iron, manganese, and zinc. Lower mean recoveries were measured for lead (84.7 percent) and nickel (84.8 percent). A higher mean recovery was measured for chromium (119 percent), with 2 of the 12 analyses having concentrations less than the minimum reporting level. With the exception of chromium and lead, both of which had low certified concentrations $(0.77 \mu \mathrm{g} / \mathrm{g}$ and $0.35 \mu \mathrm{g} / \mathrm{g}$, respectively) in the SRM, the range of variation generally was small (less than about 6 percent) for the 95-percent confidence interval, thereby indicating good reproducibility of multiple analyses of the SRM. No adjustments were made to trace-element concentrations in biota samples on the basis of recovery efficiencies.

Procedural blanks for biota consisted of the same reagents used to digest and reconstitute tissue of aquatic insects and were analyzed undiluted. Analytical results of procedural blanks for biota (table 19) are reported as a liquidphase concentration, in $\mu \mathrm{g} / \mathrm{mL}$, which is equivalent to parts per million. A procedural blank was prepared and analyzed concurrently with biota samples for each site. Concentrations of trace elements in all procedural blanks were less than the minimum reporting level; thus, no contamination bias was indicated and no adjustments to the data were necessary.

\section{Statistical Summaries of Data}

Statistical summaries of water-quality, bed-sediment, and biological data are provided in tables 20 through 22 for the period of record at each site since 1985. The summaries include the period of record, number of samples, and maximum, minimum, mean, and median concentrations.

Statistical summaries of water-quality data (table 20) are based on results of cross-sectional samples collected periodically by the USGS during the period of record for each site. The summaries do not include data for supplemental single-vertical samples collected during several years by a contract observer at Clark Fork at Turah Bridge, near Bonner; Blackfoot River near Bonner; and Clark Fork above Missoula. Inclusion of results for supplemental samples that targeted high-flow conditions or maintenance drawdowns of Milltown Reservoir would disproportionately skew the longterm statistics at these three sites relative to the other sites in the network. Statistical summaries of fine-grained bedsediment (table 21) and biological data (table 22) are based on results of samples collected once-annually during the indicated years. Because not all sites were sampled for bed sediment 
and biota every year, the data for some sites do not represent a consecutive annual record. Sampling of bulk bed sediment has been discontinued; therefore, a statistical summary is not presented.

Statistics for bed-sediment data (table 21) are based on the mean trace-element concentrations determined for each year from the averaged results of analyses of composite samples. Therefore, the number of samples for bed sediment represents the number of years that the constituent was analyzed. In contrast, statistics for biological data (table 22) are based on individual analyses for each composite sample collected, rather than on a single mean concentration for each year. Also, the number of samples for arsenic for both bed sediment and biota is smaller than the other trace elements because it was only recently (2003) included for analysis.

Differences in the number of biota composite samples among species reflect differences in species abundance, both within and between sites and among years. As a result, the statistics for biota describe a wider range of variation in traceelement concentrations than would be evident if results from individual composite samples were averaged. The abundance of aquatic insects at a particular site in a given year limits the biomass of the sample which, in turn, may result in variable minimum reporting levels. Where minimum reporting levels vary among years, differences in concentration over time are difficult to determine, especially when a large percentage of the samples have concentrations less than minimum reporting levels.

The presence or absence of insect species at a given site can vary among years and may result in different taxa being analyzed in the long-term period of record. Because Hydropsyche insects were not sorted to the species level during 1986-89, statistics for stations sampled during those years are based on the results of all Hydropsyche species combined. At some sites, statistics for the Hydropsyche morosa group are based on the combined results for two or more species because these samples could not be clearly identified to the species level, but the individual insects had morosa characteristics.

\section{References Cited}

Axtmann, E.V., Cain, D.J., and Luoma, S.N., 1997, Effect of tributary inflows on the distribution of trace metals in fine-grained sediment and benthic insects of the Clark Fork River, Montana: Environmental Science and Technology, v. 31, no. 3, p. $750-758$.

Axtmann, E.V., and Luoma, S.N., 1991, Large scale distribution of metal contamination in the fine-grained sediment of the Clark Fork River, Montana: Applied Geochemistry, v. 6, no. 6, p. $75-88$.
Cain, D.J., Luoma, S.N., and Axtmann, E.V., 1995, Influence of gut content in immature aquatic insects on assessments of environmental metal contamination: Canadian Journal of Fisheries and Aquatic Sciences, v. 52, no. 12, p. 2736-2746.

Cain, D.J., Luoma, S.N., Carter, J.L., and Ferd, S.V., 1992, Aquatic insects as bioindicators of trace element contamination in cobble-bottom rivers and streams: Canadian Journal of Fisheries and Aquatic Sciences, v. 49, no. 10, p. 2141-2154.

Childress, C.T., Foreman, W.T., Connor, B.F., and Maloney, T.J., 1999, New reporting procedures based on long-term method detection levels and some considerations for interpretations of water-quality data provided by the U.S. Geological Survey National Water Quality Laboratory: U.S. Geological Survey Open-File Report 99-193, 19 p.

Dodge, K.A., Hornberger, M.I., and Axtmann, E.V., 1996, Water-quality, bed-sediment, and biological data (October 1994 through September 1995) and statistical summaries of data for streams in the upper Clark Fork basin, Montana: U.S. Geological Survey Open-File Report 96-432, 109 p.

Dodge, K.A., Hornberger, M.I., and Axtmann, E.V., 1997, Water-quality, bed-sediment, and biological data (October 1995 through September 1996) and statistical summaries of data for streams in the upper Clark Fork basin, Montana: U.S. Geological Survey Open-File Report 97-552, 91 p.

Dodge, K.A., Hornberger, M.I., and Axtmann, E.V., 1998, Water-quality, bed-sediment, and biological data (October 1996 through September 1997) and statistical summaries of data for streams in the upper Clark Fork basin, Montana: U.S. Geological Survey Open-File Report 98-407, 102 p.

Dodge, K.A., Hornberger, M.I., and Bouse, R.M., 1999, Water-quality, bed-sediment, and biological data (October 1997 through September 1998) and statistical summaries of data for streams in the upper Clark Fork basin, Montana: U.S. Geological Survey Open-File Report 99-251, 102 p.

Dodge, K.A., Hornberger, M.I., and David, C.P.C., 2000, Water-quality, bed-sediment, and biological data (October 1998 through September 1999) and statistical summaries of data for streams in the upper Clark Fork basin, Montana: U.S. Geological Survey Open-File Report 00-370, 102 p.

Dodge, K.A., Hornberger, M.I., and David, C.P.C., 2001, Water-quality, bed-sediment, and biological data (October 1999 through September 2000) and statistical summaries of data for streams in the upper Clark Fork basin, Montana: U.S. Geological Survey Open-File Report 01-379, 95 p. 
Dodge, K.A., Hornberger, M.I., and David, C.P.C., 2002, Water-quality, bed-sediment, and biological data (October 2000 through September 2001) and statistical summaries of data for streams in the upper Clark Fork basin, Montana: U.S. Geological Survey Open-File Report 02-358, 94 p.

Dodge, K.A., Hornberger, M.I., and Lavigne, I.R., 2003, Water-quality, bed-sediment, and biological data (October 2001 through September 2002) and statistical summaries of data for streams in the upper Clark Fork basin, Montana: U.S. Geological Survey Open-File Report 03-356, 95 p.

Dodge, K.A., Hornberger, M.I., and Lavigne, I.R., 2004, Water-quality, bed-sediment, and biological data (October 2002 through September 2003) and statistical summaries of data for streams in the upper Clark Fork basin, Montana: U.S. Geological Survey Open-File Report 2004-1340, $107 \mathrm{p}$.

Dodge, K.A., Hornberger, M.I., and Dyke, J.L., 2005, Waterquality, bed-sediment, and biological data (October 2003 through September 2004) and statistical summaries of data for streams in the upper Clark Fork basin, Montana: U.S. Geological Survey Open-File Report 2005-1356, 124 p.

Dodge, K.A., and Lambing, J.H., 2006, Quality-assurance plan for the analysis of suspended sediment by the U.S. Geological Survey in Montana: U.S. Geological Survey Open-File Report 2006-1242, 25 p. [Available online at http://pubs. water.usgs.gov/ofr2006-1242]

Edwards, T.K., and Glysson, G.D., 1999, Field methods for measurement of fluvial sediment: U.S. Geological Survey Techniques of Water-Resources Investigations, book 3, chap. C2, 89 p. [Available online at http://pubs.usgs.gov/ twri/]

Faires, L.M., 1993, Methods of analysis by the U.S. Geological Survey National Water Quality Laboratory-Determination of metals in water by inductively coupled plasma-mass spectrometry: U.S. Geological Survey Open-File Report 92-634, $28 \mathrm{p}$.

Fishman, M.J., ed., 1993, Methods of analysis by the U.S. Geological Survey National Water Quality Laboratory-Determination of inorganic and organic constituents in water and fluvial sediments: U.S. Geological Survey Open-File Report 93-125, $217 \mathrm{p}$.

Friedman, L.C., and Erdmann, D.E., 1982, Quality assurance practices for the chemical and biological analyses of water and fluvial sediments: U.S. Geological Survey Techniques of Water-Resources Investigations, book 5, chap. A6, 181 p. [Available online at http://pubs.usgs.gov/twri/]
Garbarino, J.R., 1999, Methods of analysis by the U.S. Geological Survey National Water Quality Laboratory-Determination of dissolved arsenic, boron, lithium, selenium, strontium, thallium, and vanadium using inductively coupled plasma-mass spectrometry: U.S. Geological Survey OpenFile Report 99-093, 31 p.

Garbarino, J.R., and Struzeski, T.M., 1998, Methods of analysis by the U.S. Geological Survey National Water Quality Laboratory-Determination of elements in whole-water digests using inductively coupled plasma-optical emission spectrometry and inductively coupled plasma-mass spectrometry: U.S. Geological Survey Open-File Report 98-165, $101 \mathrm{p}$.

Guy, H.P., 1969, Laboratory theory and methods for sediment analysis: U.S. Geological Survey Techniques of WaterResources Investigations, book 5, chap. C1, 58 p. [Available online at http://pubs.usgs.gov/twri/]

Helsel, D.R., and Cohn, T.A., 1988, Estimation of descriptive statistics for multiply censored water quality data: Water Resources Research, v. 24, no. 12, p. 1997-2004.

Hoffman, G.L., Fishman, M.J., and Garbarino, J.R., 1996, Methods of analysis by the U.S. Geological Survey National Water Quality Laboratory-In-bottle acid digestion of whole-water samples: U.S. Geological Survey Open-File Report 96-225, 28 p.

Hornberger, M.I., Lambing, J.H., Luoma, S.N., and Axtmann, E.V., 1997, Spatial and temporal trends of trace metals in surface water, bed sediment, and biota of the upper Clark Fork basin, Montana, 1985-95: U.S. Geological Survey Open-File Report 97-669, 84 p.

Horowitz, A.J., Demas, C.R., Fitzgerald, K.K., Miller, T.L., and Rickert, D.A., 1994, U.S. Geological Survey protocol for the collection and processing of surface-water samples for the subsequent determination of inorganic constituents in filtered water: U.S. Geological Survey Open-File Report 94-539, $57 \mathrm{p}$.

Jones, B.E., 1987, Quality control manual of the U.S. Geological Survey's National Water Quality Laboratory: U.S. Geological Survey Open-File Report 87-457, 17 p.

Jones, S.R., and Garbarino, J.R., 1999, Methods of analysis by the U.S. Geological Survey National Water Quality Laboratory-Determination of arsenic and selenium in water and sediment by graphite furnace-atomic absorption spectrometry: U.S. Geological Survey Open-File Report 98-639, 39 p. 
Knapton, J.R., and Nimick, D.A., 1991, Quality assurance for water-quality activities of the U.S. Geological Survey in Montana: U.S. Geological Survey Open-File Report 91-216, $41 \mathrm{p}$.

Lambing, J.H., 1987, Water-quality data for the Clark Fork and selected tributaries from Deer Lodge to Milltown, Montana, March 1985 through June 1986: U.S. Geological Survey Open-File Report 87-110, 48 p.

Lambing, J.H., 1988, Water-quality data (July 1986 through September 1987) and statistical summaries (March 1985 through September 1987) for the Clark Fork and selected tributaries from Deer Lodge to Missoula, Montana: U.S. Geological Survey Open-File Report 88-308, 55 p.

Lambing, J.H., 1989, Water-quality data (October 1987 through September 1988) and statistical summaries (March 1985 through September 1988) for the Clark Fork and selected tributaries from Galen to Missoula, Montana: U.S. Geological Survey Open-File Report 89-229, 51 p.

Lambing, J.H., 1990, Water-quality data (October 1988 through September 1989) and statistical summaries (March 1985 through September 1989) for the Clark Fork and selected tributaries from Galen to Missoula, Montana: U.S. Geological Survey Open-File Report 90-168, 68 p.

Lambing, J.H., 1991, Water-quality and transport characteristics of suspended sediment and trace elements in streamflow of the upper Clark Fork basin from Galen to Missoula, Montana, 1985-90: U.S. Geological Survey Water-Resources Investigations Report 91-4139, 73 p.

Lambing, J.H., Hornberger, M.I., Axtmann, E.V., and Dodge, K.A., 1995, Water-quality, bed-sediment, and biological data (October 1993 through September 1994) and statistical summaries of data for streams in the upper Clark Fork basin, Montana: U.S. Geological Survey Open-File Report 95-429, 104 p.
Lambing, J.H., Hornberger, M.I., Axtmann, E.V., and Pope, D.A., 1994, Water-quality, bed-sediment, and biological data (October 1992 through September 1993) and statistical summaries of water-quality data (March 1985 through September 1993) for streams in the upper Clark Fork basin, Montana: U.S. Geological Survey Open-File Report 94-375, $85 \mathrm{p}$.

Luoma, S.N., and Bryan, G.W., 1981, A statistical assessment of the form of trace metals in oxidized estuarine sediments employing chemical extractants: Science of the Total Environment, v. 17, no. 17, p. 167-196.

Porterfield, George, 1972, Computation of fluvial-sediment discharge: U.S. Geological Survey Techniques of WaterResources Investigations, book 3, chap. C3, 66 p. [Available online at http://pubs.usgs.gov/twri/]

Pritt, J.W., and Raese, J.W., eds., 1995, Quality assurance/ quality control manual-National Water Quality Laboratory: U.S. Geological Survey Open-File Report 95-443, $35 \mathrm{p}$.

Rantz, S.E., and others, 1982, Measurement and computation of streamflow: U.S. Geological Survey Water-Supply Paper 2175, 2 v., $631 \mathrm{p}$.

Taylor, J.K., 1987, Quality assurance of chemical measurements: Chelsea, Mich., Lewis Publishers, 328 p.

U.S. Geological Survey, variously dated, National field manual for the collection of water-quality data: U.S. Geological Survey Techniques of Water-Resources Investigations, book 9, chaps. A1-A9. [Available online at http://pubs.usgs. gov/twri/]

Ward, J.R., and Harr, C.A., eds., 1990, Methods for collection and processing of surface-water and bed-material samples for physical and chemical analyses: U.S. Geological Survey Open-File Report 90-140, 71 p. 
Data 
Table 4. Water-quality data for the upper Clark Fork basin, Montana, October 2004 through September 2005.

[Abbreviations: $\mathrm{ft} \mathrm{t}^{3} / \mathrm{s}$, cubic feet per second; ${ }^{\circ} \mathrm{C}$, degrees Celsius; E, estimated; $\mu \mathrm{g} / \mathrm{L}$, micrograms per liter; $\mu \mathrm{S} / \mathrm{cm}$, microsiemens per centimeter at $25^{\circ} \mathrm{C}$; $\mathrm{mg} / \mathrm{L}$, milligrams per liter; mm, millimeters; ton/d, tons per day. Symbol: $<$, less than laboratory reporting level]

\begin{tabular}{|c|c|c|c|c|c|c|c|c|}
\hline \multicolumn{9}{|c|}{ 12323230 —Blacktail Creek at Harrison Avenue, at Butte } \\
\hline $12 / 15 / 2004$ & 0900 & 7.7 & 326 & 5.5 & 130 & 36.2 & 9.00 & 2.0 \\
\hline $04 / 18 / 2005$ & 0910 & 7.6 & 264 & 5.0 & 100 & 29.4 & 7.29 & 2.6 \\
\hline 05/16/2005 & 0900 & 7.7 & 203 & 9.5 & 74 & 21.2 & 5.03 & 5.8 \\
\hline 06/01/2005 & 1120 & 7.7 & 161 & 9.0 & 65 & 19.3 & 4.09 & 5.0 \\
\hline 06/13/2005 & 1030 & 7.8 & 200 & 8.5 & 83 & 24.6 & 5.23 & 5.9 \\
\hline Date & $\begin{array}{c}\text { Arsenic, } \\
\text { unfiltered } \\
\text { recoverable } \\
\text { ( } \mu \mathrm{g} / \mathrm{L})\end{array}$ & $\begin{array}{c}\text { Cadmium } \\
\text { filtered } \\
\text { ( } \mu \mathrm{g} / \mathrm{L})\end{array}$ & $\begin{array}{c}\text { Cadmium } \\
\text { unfiltered } \\
\text { recoverable } \\
\text { ( } \mu \mathrm{g} / \mathrm{L})\end{array}$ & $\begin{array}{c}\text { Copper, } \\
\text { filtered } \\
\text { ( } \mu \mathrm{g} / \mathrm{L})\end{array}$ & $\begin{array}{c}\text { Copper, } \\
\text { unfiltered } \\
\text { recoverable } \\
\text { ( } \mu \mathrm{g} / \mathrm{L})\end{array}$ & $\begin{array}{c}\text { Iron, } \\
\text { filtered } \\
\text { ( } \mu \mathrm{g} / \mathrm{L})\end{array}$ & $\begin{array}{c}\text { Iron, } \\
\text { unfiltered } \\
\text { recoverable } \\
\text { ( } \mu \mathrm{g} / \mathrm{L} \text { ) }\end{array}$ & $\begin{array}{c}\text { Lead, } \\
\text { filtered } \\
\text { ( } \mathrm{gg} / \mathrm{L})\end{array}$ \\
\hline $12 / 15 / 2004$ & 2 & 0.07 & 0.07 & 8.9 & 8.0 & 49 & 1,030 & 2.80 \\
\hline 03/09/2005 & E1. & E.03 & E.04 & .9 & 3.4 & 54 & 290 & $<.08$ \\
\hline $04 / 18 / 2005$ & 4 & .05 & .07 & 3.4 & 7.2 & 201 & 690 & .19 \\
\hline $05 / 16 / 2005$ & 7 & .04 & .05 & 8.5 & 12.4 & 179 & 540 & .24 \\
\hline 06/01/2005 & 7 & .06 & .07 & 7.5 & 14.2 & 143 & 810 & .25 \\
\hline $12 / 15 / 2004$ & 2.74 & 26.4 & 55 & 2.2 & 11 & 89 & 22 & 0.20 \\
\hline 03/09/2005 & .41 & 28.7 & 29 & 3.2 & 5 & 83 & 8 & .06 \\
\hline $04 / 18 / 2005$ & 1.24 & 29.2 & 46 & 4.2 & 12 & 85 & 9 & .17 \\
\hline 05/16/2005 & .92 & 19.0 & 28 & 3.9 & 7 & 54 & 13 & .77 \\
\hline 06/01/2005 & 2.67 & 23.1 & 51 & 6.2 & 18 & 71 & 24 & 1.6 \\
\hline $06 / 13 / 2005$ & .62 & 14.2 & 23 & 3.2 & 4 & 71 & 7 & .38 \\
\hline $07 / 25 / 2005$ & .26 & 32.5 & 38 & 3.7 & 5 & 87 & 3 & .02 \\
\hline $08 / 23 / 2005$ & .61 & 35.4 & 47 & 2.2 & 5 & 83 & 9 & .06 \\
\hline
\end{tabular}


Table 4. Water-quality data for the upper Clark Fork basin, Montana, October 2004 through September 2005.-Continued

[Abbreviations: $\mathrm{ft} 3 / \mathrm{s}$, cubic feet per second; ${ }^{\circ} \mathrm{C}$, degrees Celsius; E, estimated; $\mu \mathrm{g} / \mathrm{L}$, micrograms per liter; $\mu \mathrm{S} / \mathrm{cm}$, microsiemens per centimeter at $25^{\circ} \mathrm{C}$; $\mathrm{mg} / \mathrm{L}$, milligrams per liter; mm, millimeters; ton/d, tons per day. Symbol: <, less than laboratory reporting level]

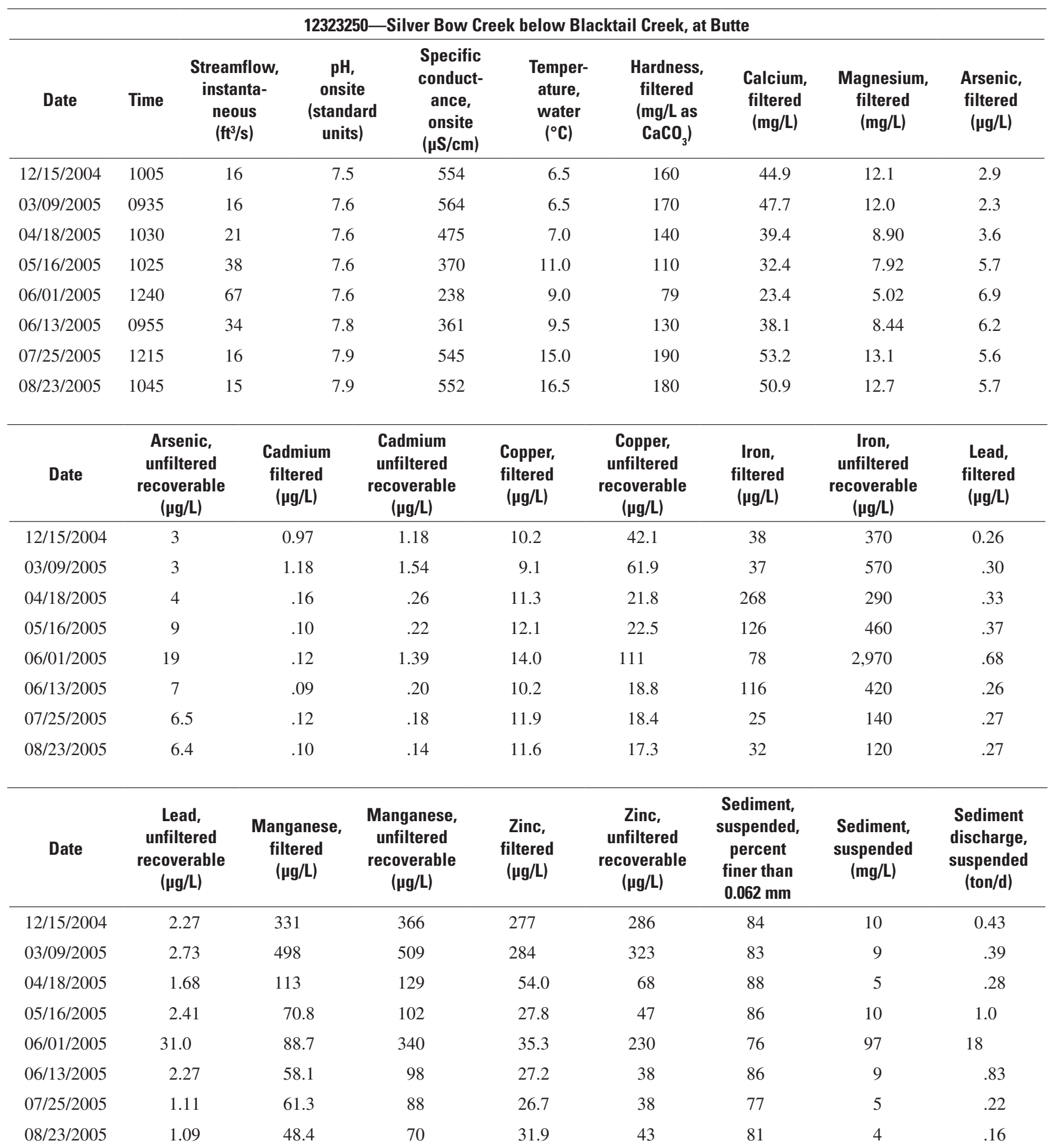


Table 4. Water-quality data for the upper Clark Fork basin, Montana, October 2004 through September 2005.—Continued

[Abbreviations: $\mathrm{ft} 3 / \mathrm{s}$, cubic feet per second; ${ }^{\circ} \mathrm{C}$, degrees Celsius; E, estimated; $\mu \mathrm{g} / \mathrm{L}$, micrograms per liter; $\mu \mathrm{S} / \mathrm{cm}$, microsiemens per centimeter at $25^{\circ} \mathrm{C}$; $\mathrm{mg} / \mathrm{L}$, milligrams per liter; mm, millimeters; ton/d, tons per day. Symbol: $<$, less than laboratory reporting level]

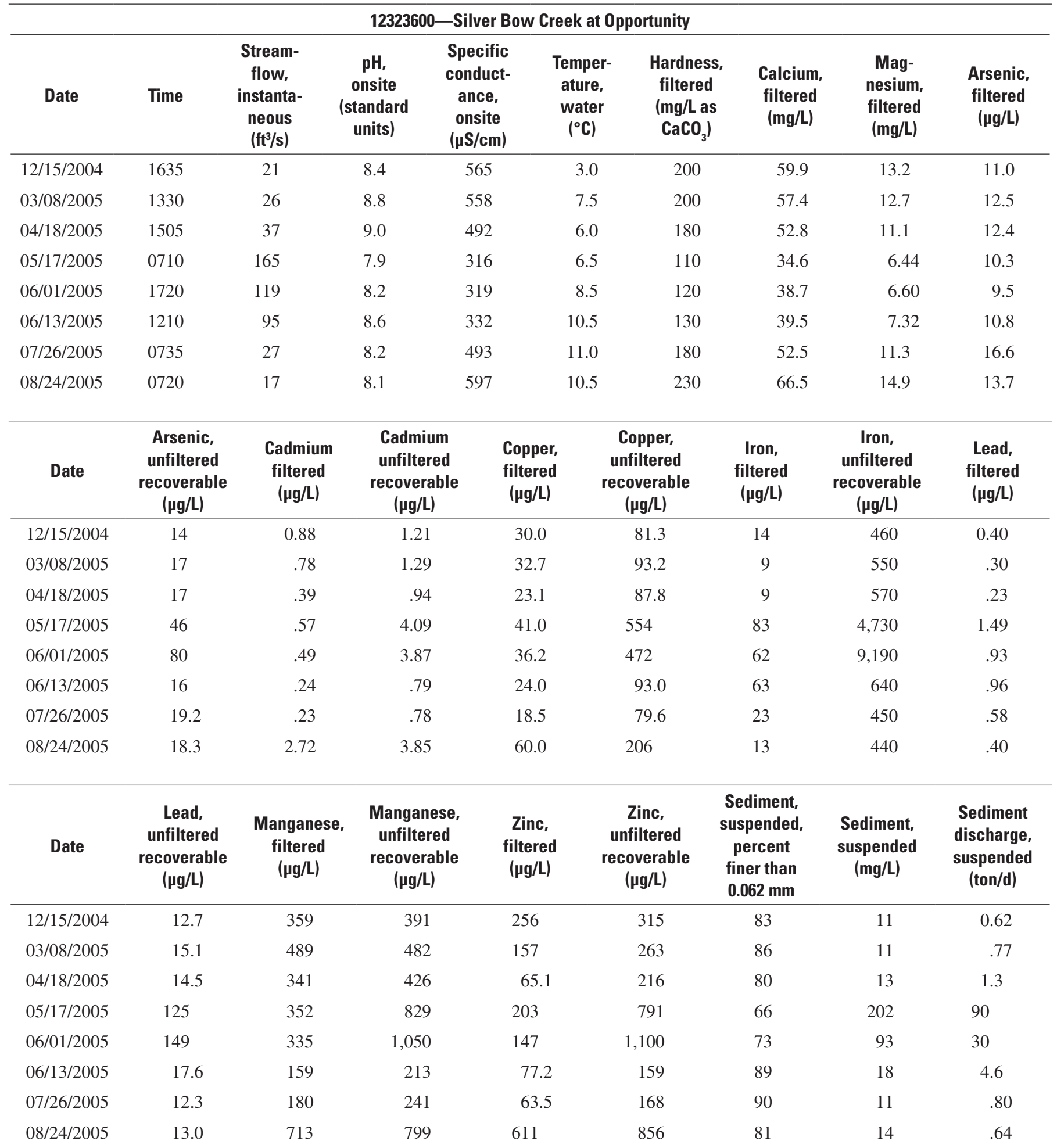


Table 4. Water-quality data for the upper Clark Fork basin, Montana, October 2004 through September 2005.-Continued

[Abbreviations: $\mathrm{ft}{ }^{3} / \mathrm{s}$, cubic feet per second; ${ }^{\circ} \mathrm{C}$, degrees Celsius; E, estimated; $\mu \mathrm{g} / \mathrm{L}$, micrograms per liter; $\mu \mathrm{S} / \mathrm{cm}$, microsiemens per centimeter at $25^{\circ} \mathrm{C}$; $\mathrm{mg} / \mathrm{L}$, milligrams per liter; mm, millimeters; ton/d, tons per day. Symbol: <, less than laboratory reporting level]

\begin{tabular}{|c|c|c|c|c|c|c|c|c|c|}
\hline \multicolumn{10}{|c|}{ 12323670_Mill Creek near Anaconda } \\
\hline $12 / 15 / 2004$ & 1330 & 10 & 8.3 & 179 & 3.0 & 82 & 21.7 & 6.75 & 9.8 \\
\hline $04 / 18 / 2005$ & 1155 & 14 & 8.2 & 161 & 4.0 & 72 & 19.3 & 5.71 & 11.3 \\
\hline $05 / 16 / 2005$ & 1315 & 113 & 7.7 & 86 & 8.0 & 33 & 9.40 & 2.25 & 19.1 \\
\hline 06/01/2005 & 1410 & 153 & 8.0 & 67 & 6.0 & 28 & 8.29 & 1.83 & 11.7 \\
\hline $06 / 22 / 2005$ & 1145 & 165 & 7.8 & 56 & 9.5 & 24 & 7.12 & 1.45 & 7.3 \\
\hline Date & $\begin{array}{c}\text { Arsenic, } \\
\text { unfiltered } \\
\text { recoverable } \\
(\mu \mathrm{g} / \mathrm{L})\end{array}$ & $\begin{array}{c}\text { Cadmium } \\
\text { filtered } \\
\text { ( } \mu \mathrm{g} / \mathrm{L})\end{array}$ & $\begin{array}{c}\text { Cadmium } \\
\text { unfiltered } \\
\text { recoverable } \\
(\mu \mathrm{g} / \mathrm{L})\end{array}$ & & & $\begin{array}{l}\text { Copper, } \\
\text { unfiltered } \\
\text { ecoverable } \\
(\mu \mathrm{g} / \mathrm{L})\end{array}$ & $\begin{array}{c}\text { Iron, } \\
\text { filtered } \\
\text { ( } \mu \mathrm{g} / \mathrm{L})\end{array}$ & $\begin{array}{c}\text { Iron, } \\
\text { unfiltered } \\
\text { recoverable } \\
(\mu \mathrm{g} / \mathrm{L})\end{array}$ & $\begin{array}{c}\text { Lead, } \\
\text { filtered } \\
\text { ( } \mathrm{gg} / \mathrm{L})\end{array}$ \\
\hline $12 / 15 / 2004$ & 10 & 0.08 & 0.05 & & & 1.3 & 41 & 90 & 0.17 \\
\hline 03/08/2005 & 9 & .11 & .05 & & & 1.9 & 27 & 90 & E.05 \\
\hline $04 / 18 / 2005$ & 13 & .04 & .06 & & & 2.8 & 42 & 130 & .10 \\
\hline $05 / 16 / 2005$ & 25 & .05 & .18 & & & 10.3 & 62 & 620 & .24 \\
\hline $06 / 01 / 2005$ & 15 & .04 & .08 & & & 5.7 & 35 & 170 & .11 \\
\hline $12 / 15 / 2004$ & 0.27 & 6.4 & 7 & & & E1 & 81 & 1 & 0.03 \\
\hline 03/08/2005 & .31 & 7.0 & 9 & & & E1 & 75 & 2 & .04 \\
\hline $04 / 18 / 2005$ & .54 & 5.8 & 13 & & & E2 & 78 & 2 & .08 \\
\hline $05 / 16 / 2005$ & 3.12 & 5.9 & 37 & & & 8 & 64 & 29 & 8.8 \\
\hline 06/01/2005 & .83 & 3.6 & 11 & & & 4 & 51 & 6 & 2.5 \\
\hline $06 / 22 / 2005$ & 2.35 & 5.1 & 36 & & & 7 & 57 & 29 & 13 \\
\hline $07 / 25 / 2005$ & .59 & 8.9 & 17 & & & 3 & 79 & 2 & .15 \\
\hline $08 / 23 / 2005$ & .57 & 6.8 & 18 & & & E2 & 75 & 3 & .10 \\
\hline
\end{tabular}


Table 4. Water-quality data for the upper Clark Fork basin, Montana, October 2004 through September 2005.-Continued

[Abbreviations: $\mathrm{ft} / \mathrm{s}$, cubic feet per second; ${ }^{\circ} \mathrm{C}$, degrees Celsius; E, estimated; $\mu \mathrm{g} / \mathrm{L}$, micrograms per liter; $\mu \mathrm{S} / \mathrm{cm}$, microsiemens per centimeter at $25^{\circ} \mathrm{C}$; $\mathrm{mg} / \mathrm{L}$, milligrams per liter; mm, millimeters; ton/d, tons per day. Symbol: $<$, less than laboratory reporting level]

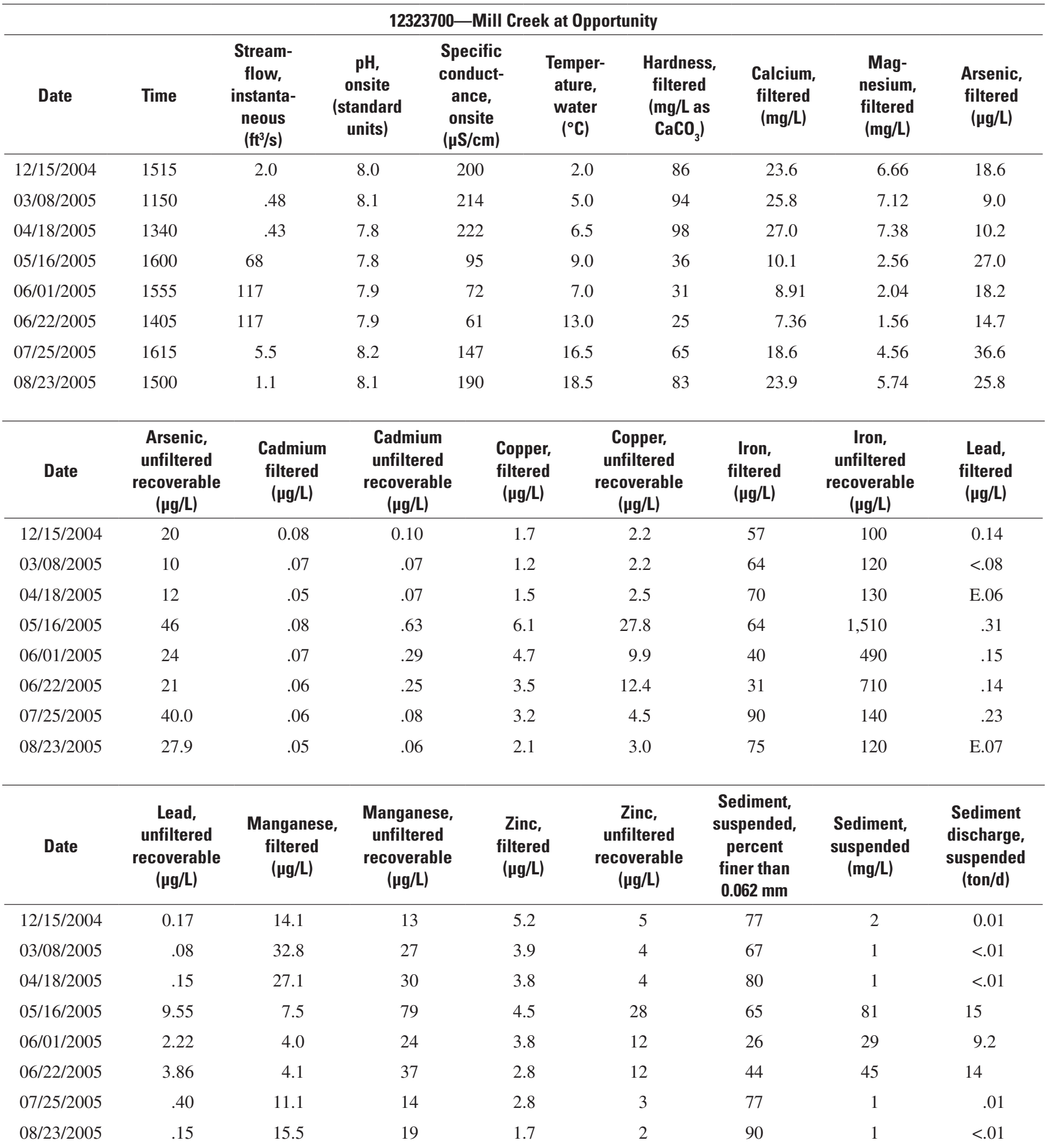


Table 4. Water-quality data for the upper Clark Fork basin, Montana, October 2004 through September 2005.-Continued

[Abbreviations: $\mathrm{ft} 3 / \mathrm{s}$, cubic feet per second; ${ }^{\circ} \mathrm{C}$, degrees Celsius; E, estimated; $\mu \mathrm{g} / \mathrm{L}$, micrograms per liter; $\mu \mathrm{S} / \mathrm{cm}$, microsiemens per centimeter at $25^{\circ} \mathrm{C}$; $\mathrm{mg} / \mathrm{L}$, milligrams per liter; mm, millimeters; ton/d, tons per day. Symbol: $<$, less than laboratory reporting level]

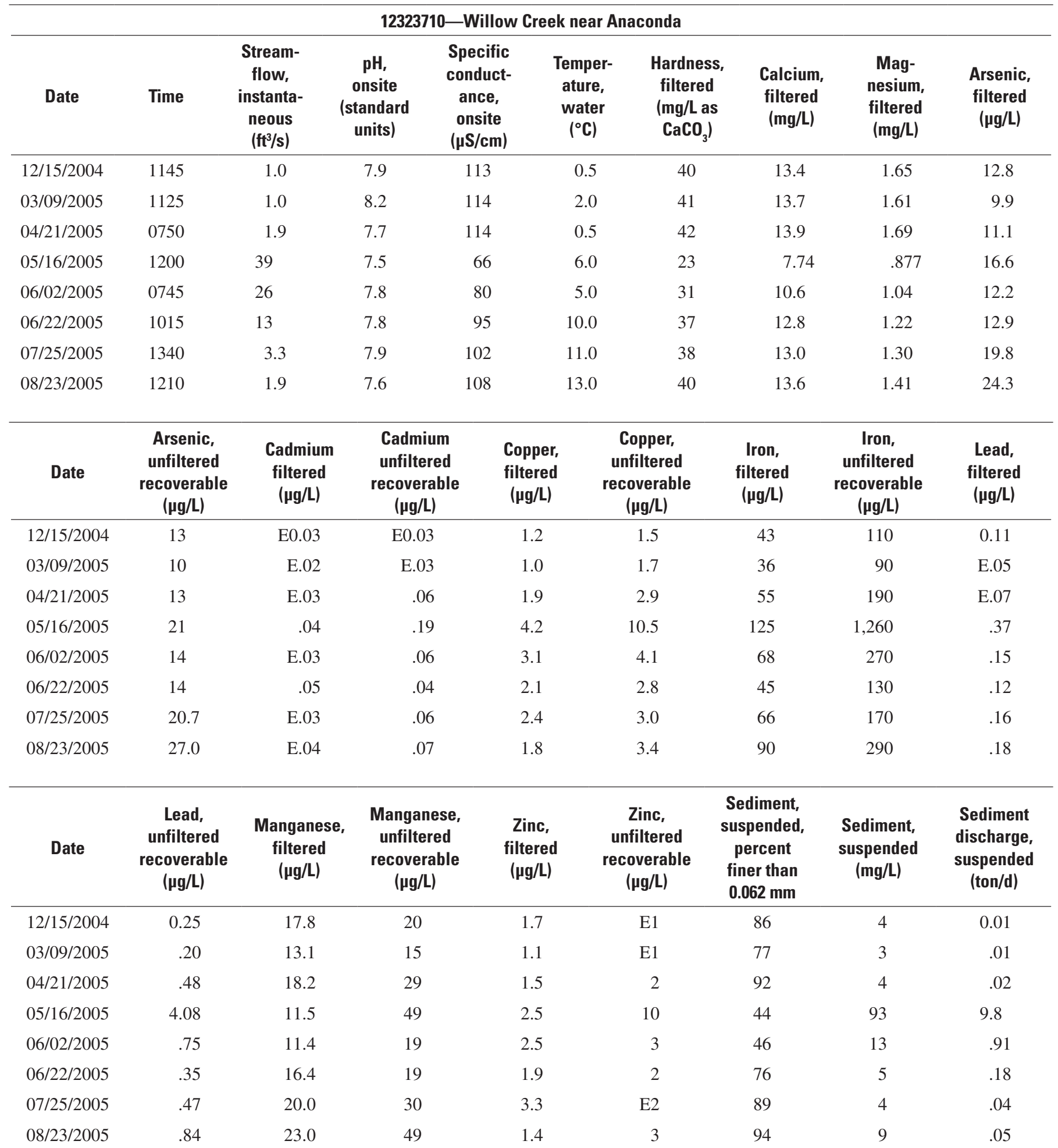


Table 4. Water-quality data for the upper Clark Fork basin, Montana, October 2004 through September 2005.-Continued

[Abbreviations: $\mathrm{ft} \mathrm{t}^{3} / \mathrm{s}$, cubic feet per second; ${ }^{\circ} \mathrm{C}$, degrees Celsius; E, estimated; $\mu \mathrm{g} / \mathrm{L}$, micrograms per liter; $\mu \mathrm{S} / \mathrm{cm}$, microsiemens per centimeter at $25^{\circ} \mathrm{C}$; $\mathrm{mg} / \mathrm{L}$, milligrams per liter; $\mathrm{mm}$, millimeters; ton/d, tons per day. Symbol: <, less than laboratory reporting level]

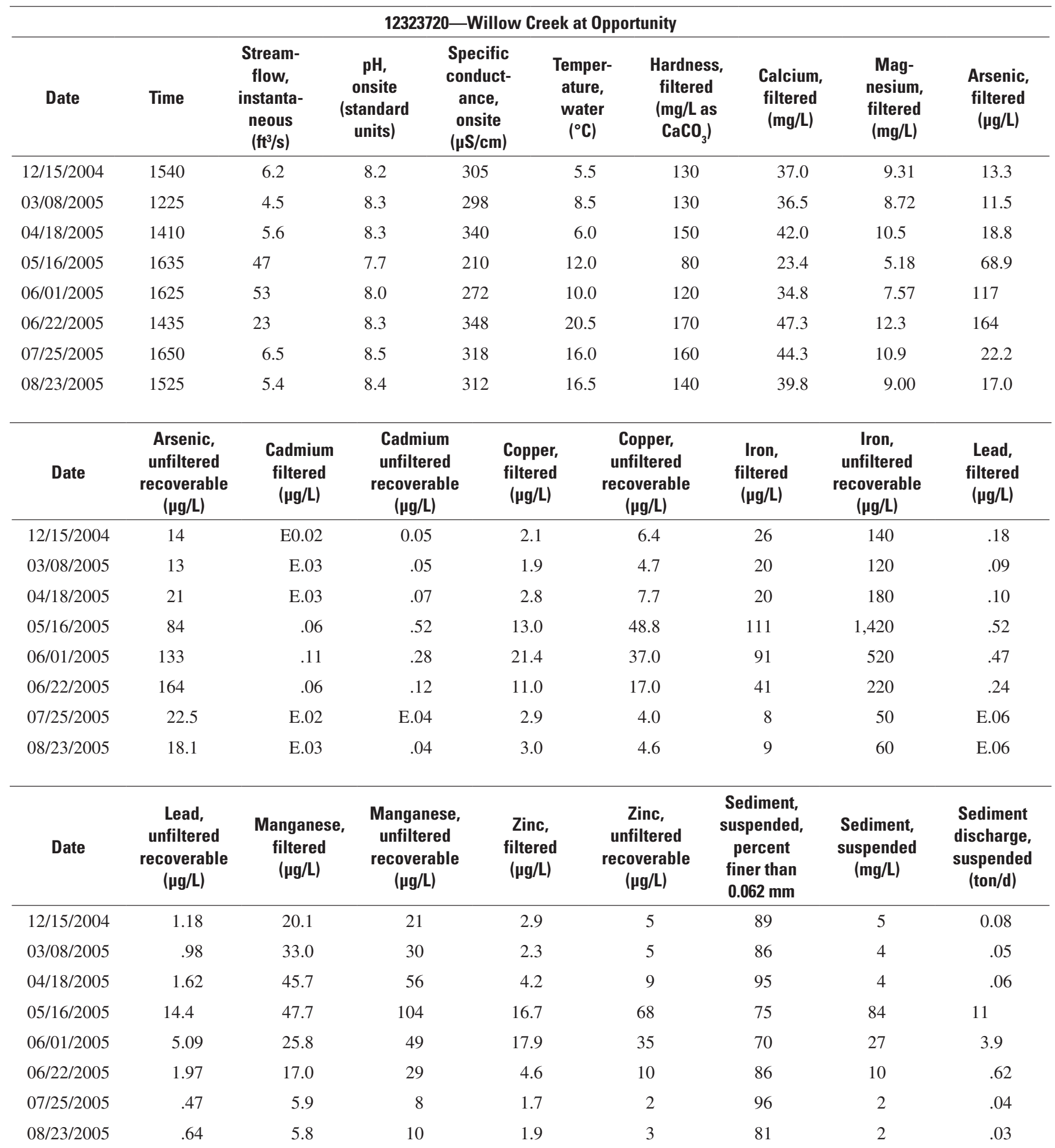


Table 4. Water-quality data for the upper Clark Fork basin, Montana, October 2004 through September 2005.-Continued

[Abbreviations: $\mathrm{ft} 3 / \mathrm{s}$, cubic feet per second; ${ }^{\circ} \mathrm{C}$, degrees Celsius; E, estimated; $\mu \mathrm{g} / \mathrm{L}$, micrograms per liter; $\mu \mathrm{S} / \mathrm{cm}$, microsiemens per centimeter at $25^{\circ} \mathrm{C}$; $\mathrm{mg} / \mathrm{L}$, milligrams per liter; mm, millimeters; ton/d, tons per day. Symbol: $<$, less than laboratory reporting level]

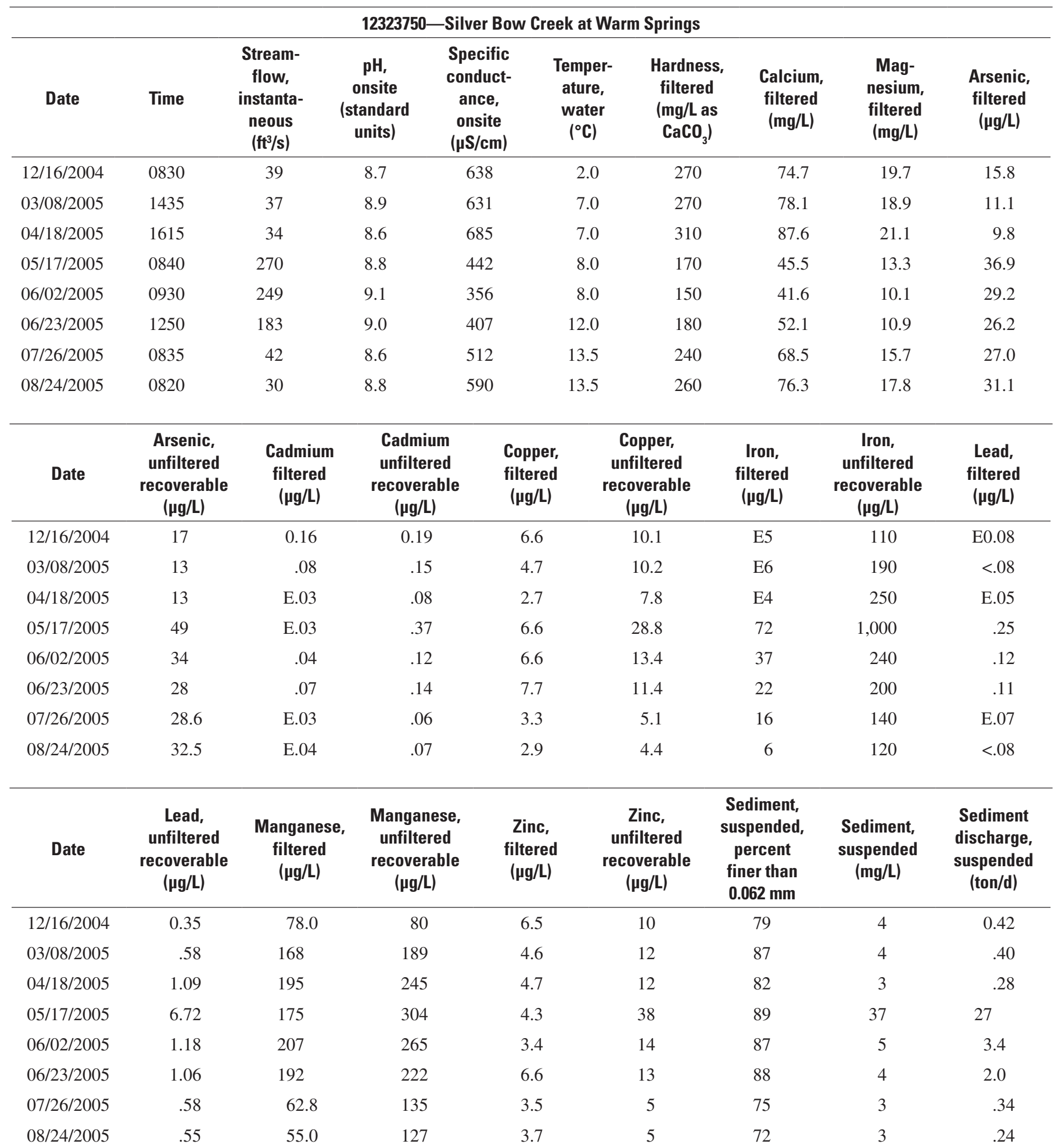


Table 4. Water-quality data for the upper Clark Fork basin, Montana, October 2004 through September 2005.—Continued

[Abbreviations: $\mathrm{ft} / \mathrm{s}$, cubic feet per second; ${ }^{\circ} \mathrm{C}$, degrees Celsius; E, estimated; $\mu \mathrm{g} / \mathrm{L}$, micrograms per liter; $\mu \mathrm{S} / \mathrm{cm}$, microsiemens per centimeter at $25^{\circ} \mathrm{C}$; $\mathrm{mg} / \mathrm{L}$, milligrams per liter; mm, millimeters; ton/d, tons per day. Symbol: <, less than laboratory reporting level]

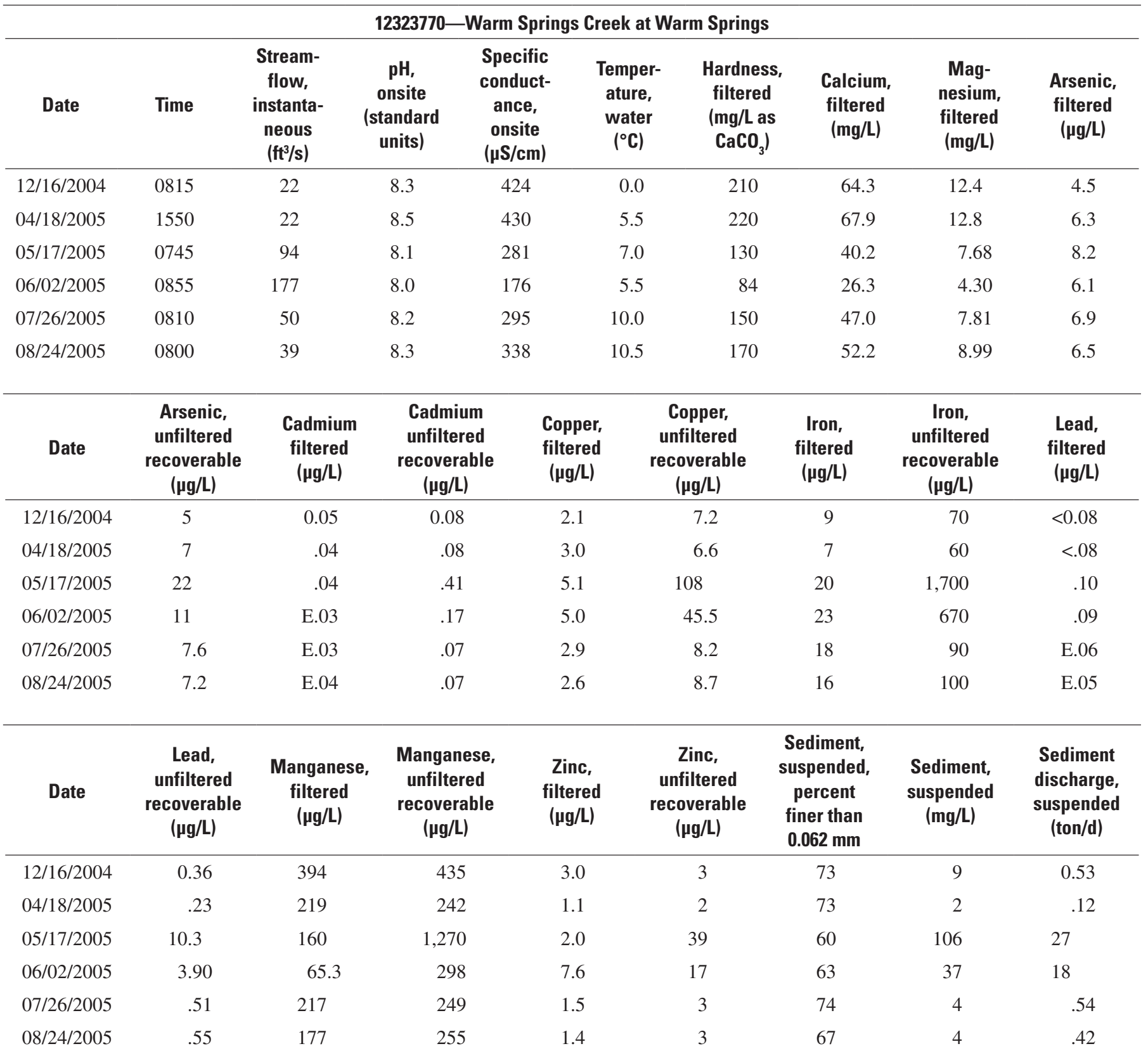


Table 4. Water-quality data for the upper Clark Fork basin, Montana, October 2004 through September 2005.-Continued

[Abbreviations: $\mathrm{ft} 3 / \mathrm{s}$, cubic feet per second; ${ }^{\circ} \mathrm{C}$, degrees Celsius; E, estimated; $\mu \mathrm{g} / \mathrm{L}$, micrograms per liter; $\mu \mathrm{S} / \mathrm{cm}$, microsiemens per centimeter at $25^{\circ} \mathrm{C}$; $\mathrm{mg} / \mathrm{L}$, milligrams per liter; mm, millimeters; ton/d, tons per day. Symbol: $<$, less than laboratory reporting level]

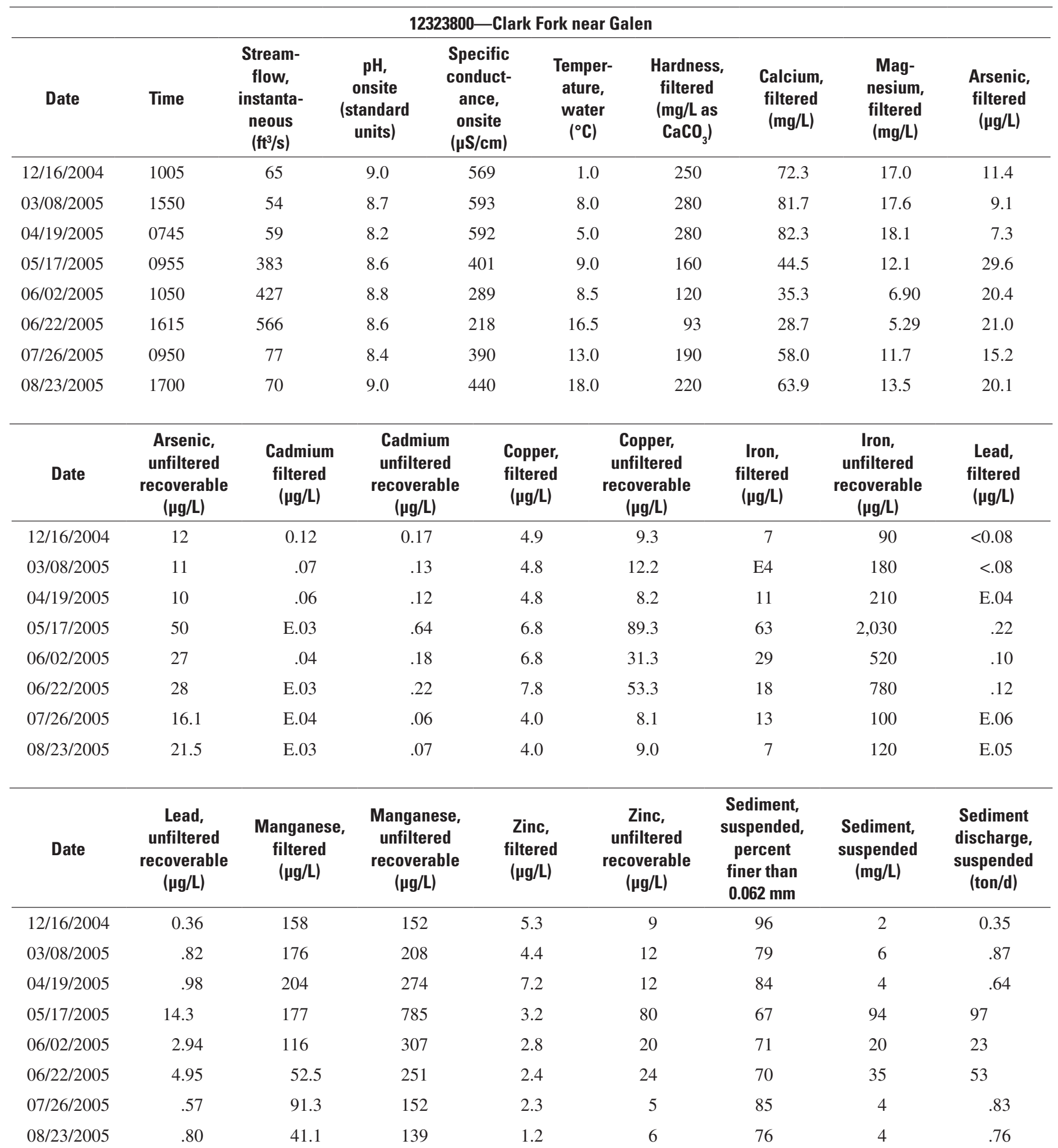


Table 4. Water-quality data for the upper Clark Fork basin, Montana, October 2004 through September 2005.—Continued

[Abbreviations: $\mathrm{ft} \mathrm{ft}^{3} / \mathrm{s}$, cubic feet per second; ${ }^{\circ} \mathrm{C}$, degrees Celsius; E, estimated; $\mu \mathrm{g} / \mathrm{L}$, micrograms per liter; $\mu \mathrm{S} / \mathrm{cm}$, microsiemens per centimeter at $25^{\circ} \mathrm{C}$; $\mathrm{mg} / \mathrm{L}$, milligrams per liter; mm, millimeters; ton/d, tons per day. Symbol: $<$, less than laboratory reporting level]

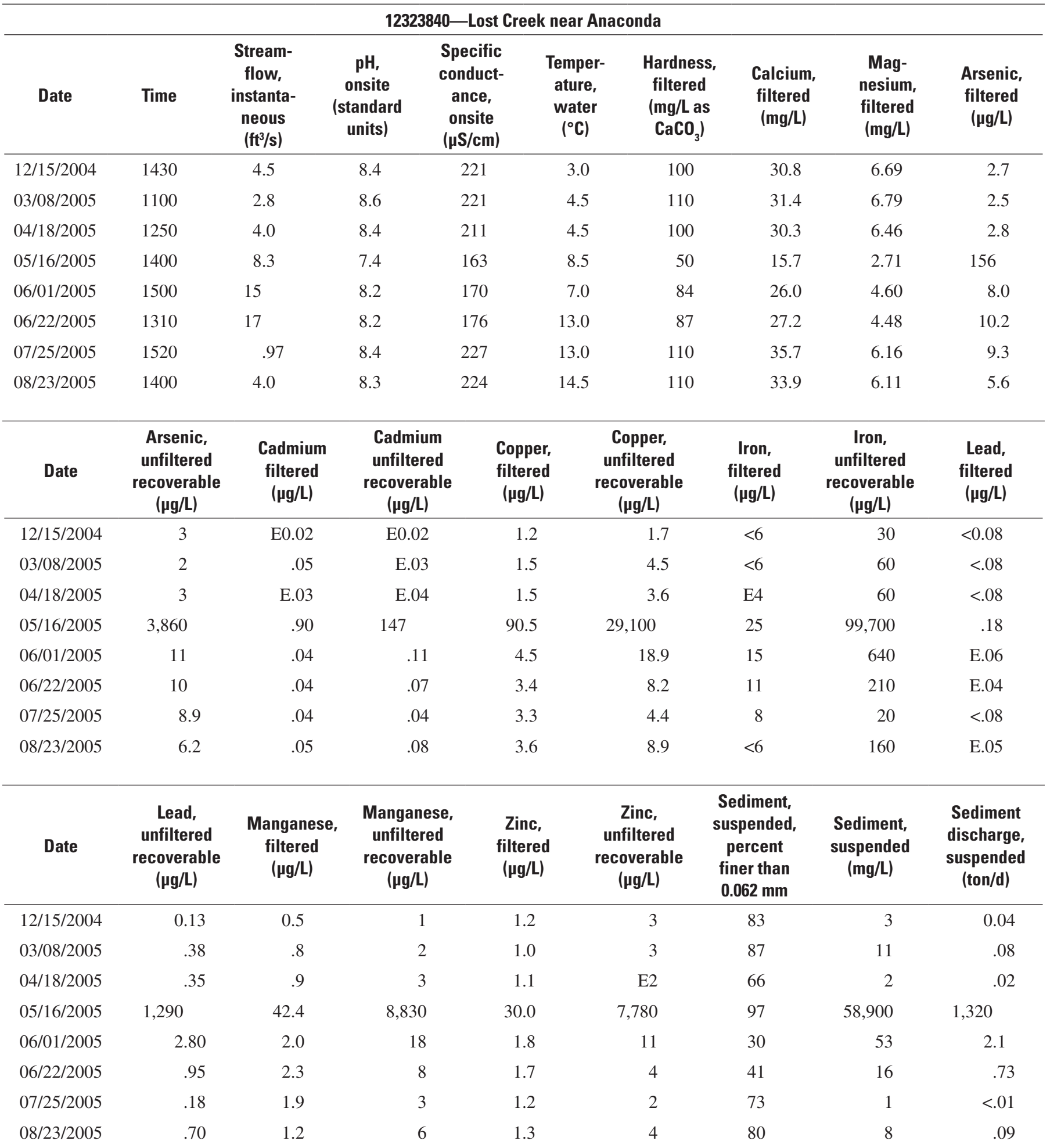


Table 4. Water-quality data for the upper Clark Fork basin, Montana, October 2004 through September 2005.—Continued

[Abbreviations: $\mathrm{ft} 3 / \mathrm{s}$, cubic feet per second; ${ }^{\circ} \mathrm{C}$, degrees Celsius; E, estimated; $\mu \mathrm{g} / \mathrm{L}$, micrograms per liter; $\mu \mathrm{S} / \mathrm{cm}$, microsiemens per centimeter at $25^{\circ} \mathrm{C}$; $\mathrm{mg} / \mathrm{L}$, milligrams per liter; mm, millimeters; ton/d, tons per day. Symbol: $<$, less than laboratory reporting level]

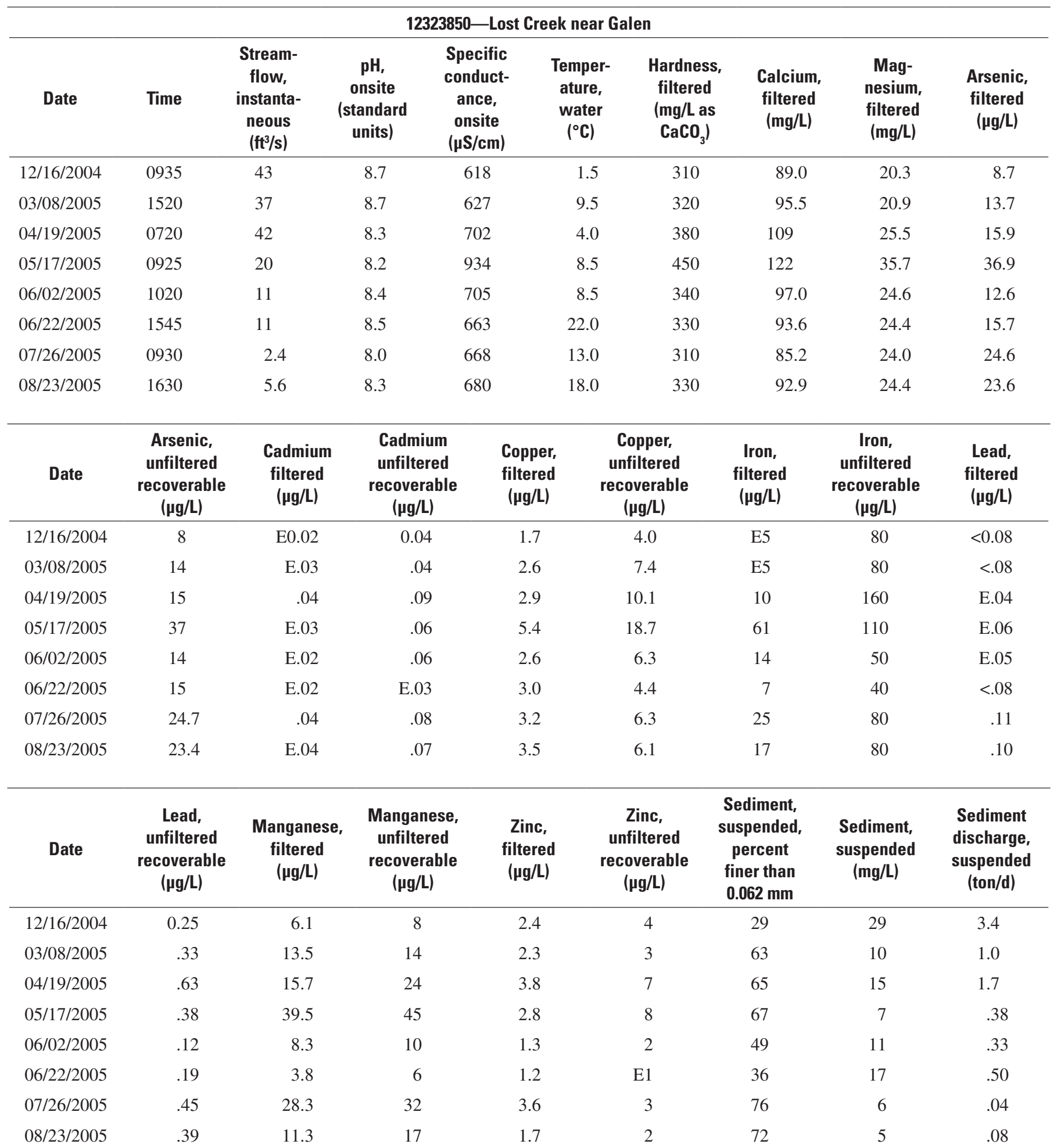


Table 4. Water-quality data for the upper Clark Fork basin, Montana, October 2004 through September 2005. - Continued

[Abbreviations: $\mathrm{ft} \mathrm{t}^{3} / \mathrm{s}$, cubic feet per second; ${ }^{\circ} \mathrm{C}$, degrees Celsius; E, estimated; $\mu \mathrm{g} / \mathrm{L}$, micrograms per liter; $\mu \mathrm{S} / \mathrm{cm}$, microsiemens per centimeter at $25^{\circ} \mathrm{C}$; $\mathrm{mg} / \mathrm{L}$, milligrams per liter; mm, millimeters; ton/d, tons per day. Symbol: $<$, less than laboratory reporting level]

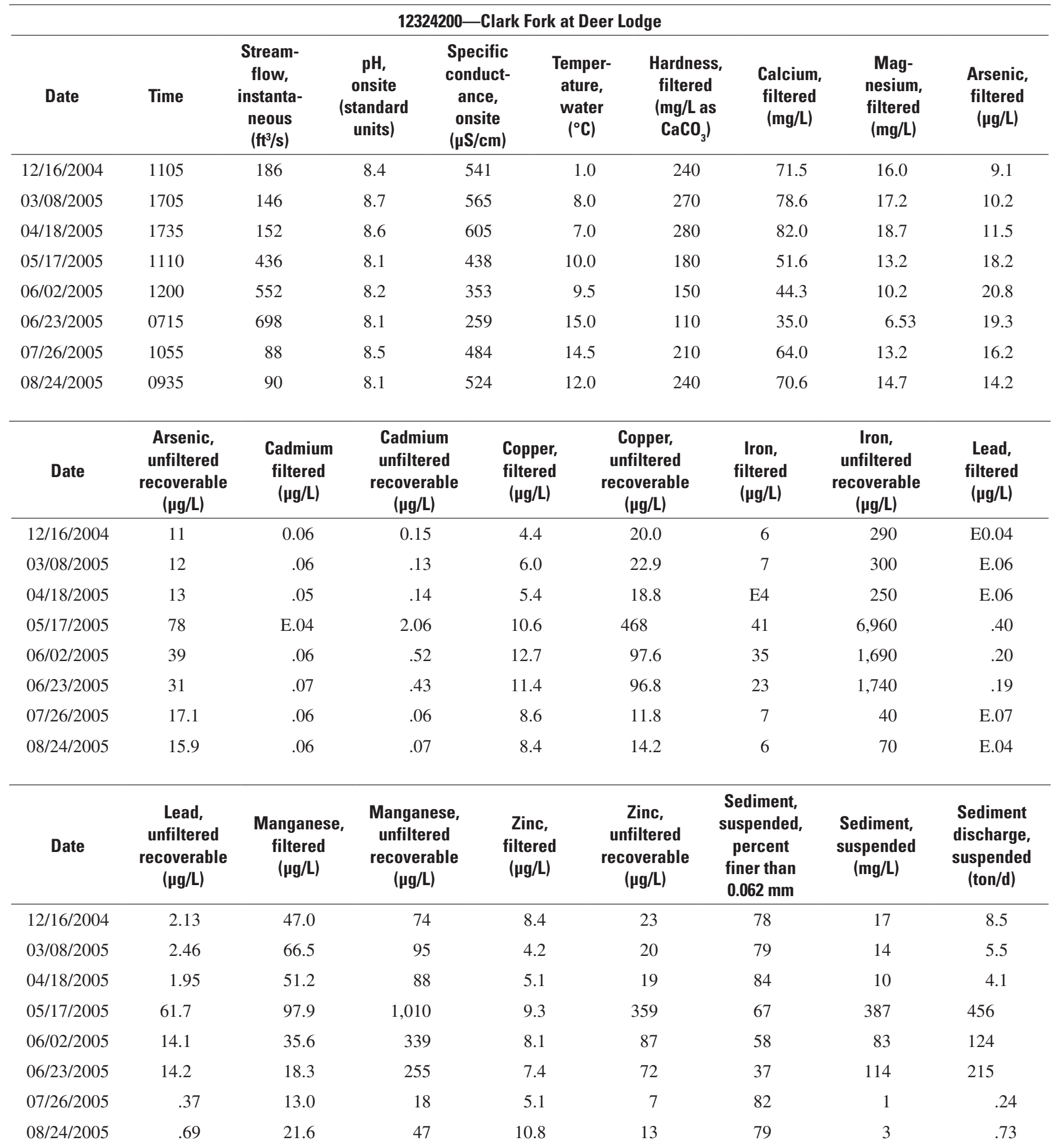


Table 4. Water-quality data for the upper Clark Fork basin, Montana, October 2004 through September 2005.-Continued

[Abbreviations: $\mathrm{ft} 3 / \mathrm{s}$, cubic feet per second; ${ }^{\circ} \mathrm{C}$, degrees Celsius; E, estimated; $\mu \mathrm{g} / \mathrm{L}$, micrograms per liter; $\mu \mathrm{S} / \mathrm{cm}$, microsiemens per centimeter at $25^{\circ} \mathrm{C}$; $\mathrm{mg} / \mathrm{L}$, milligrams per liter; mm, millimeters; ton/d, tons per day. Symbol: $<$, less than laboratory reporting level]

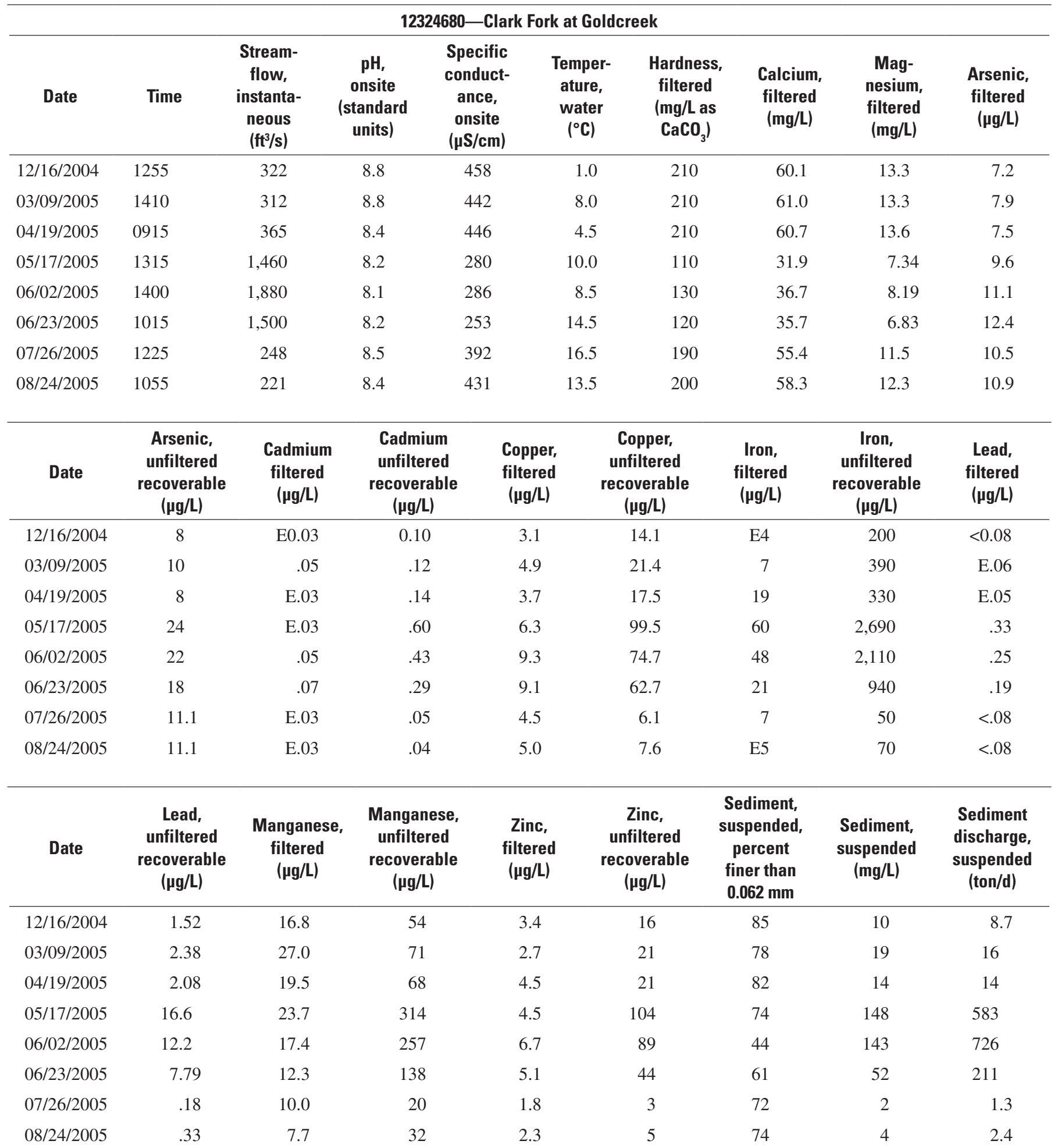


Table 4. Water-quality data for the upper Clark Fork basin, Montana, October 2004 through September 2005.-Continued

[Abbreviations: $\mathrm{ft} \mathrm{t}^{3} / \mathrm{s}$, cubic feet per second; ${ }^{\circ} \mathrm{C}$, degrees Celsius; E, estimated; $\mu \mathrm{g} / \mathrm{L}$, micrograms per liter; $\mu \mathrm{S} / \mathrm{cm}$, microsiemens per centimeter at $25^{\circ} \mathrm{C}$; $\mathrm{mg} / \mathrm{L}$, milligrams per liter; mm, millimeters; ton/d, tons per day. Symbol: $<$, less than laboratory reporting level]

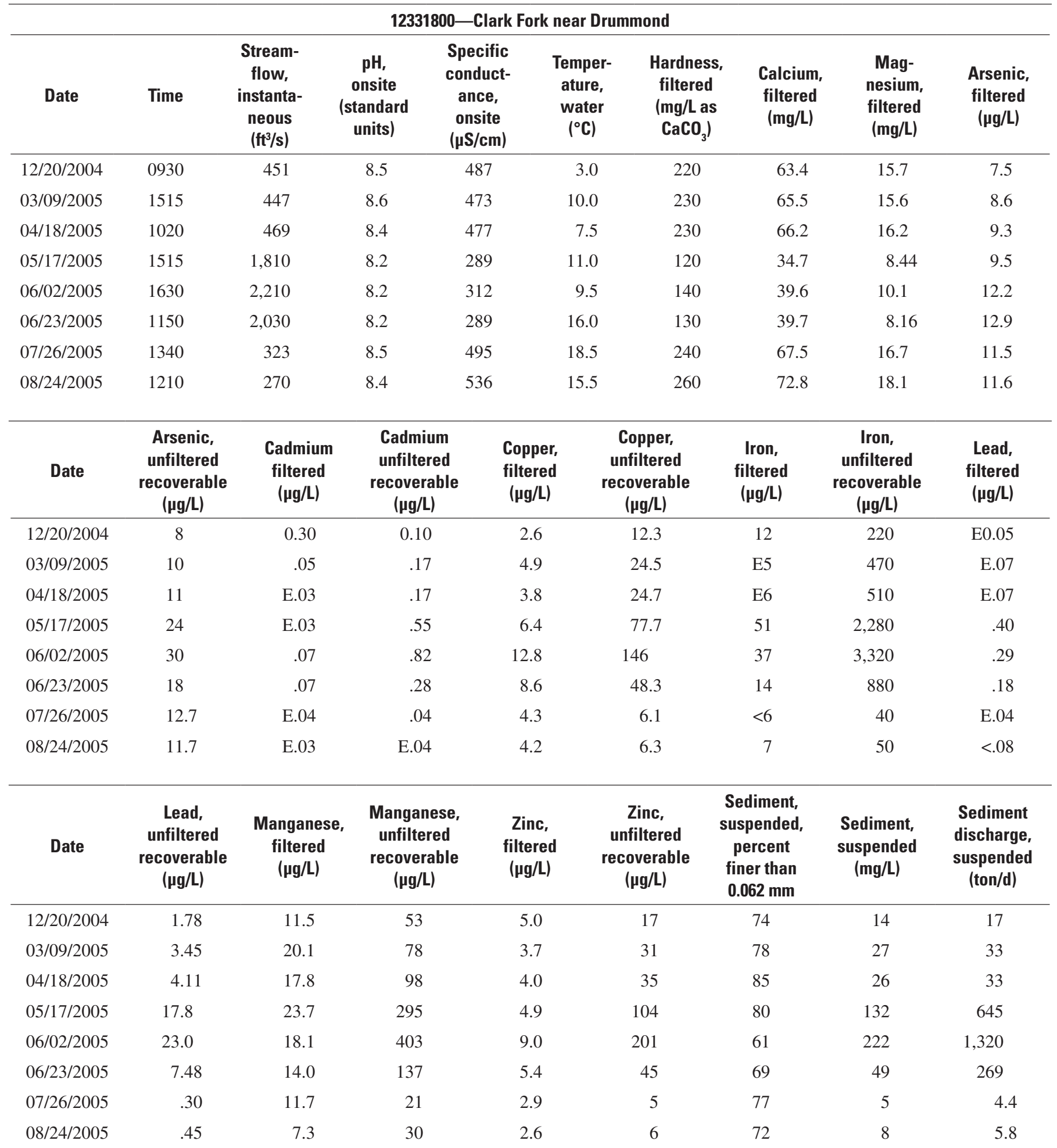


Table 4. Water-quality data for the upper Clark Fork basin, Montana, October 2004 through September 2005. - Continued

[Abbreviations: $\mathrm{ft} 3 / \mathrm{s}$, cubic feet per second; ${ }^{\circ} \mathrm{C}$, degrees Celsius; E, estimated; $\mu \mathrm{g} / \mathrm{L}$, micrograms per liter; $\mu \mathrm{S} / \mathrm{cm}$, microsiemens per centimeter at $25^{\circ} \mathrm{C}$; $\mathrm{mg} / \mathrm{L}$, milligrams per liter; mm, millimeters; ton/d, tons per day. Symbol: $<$, less than laboratory reporting level]

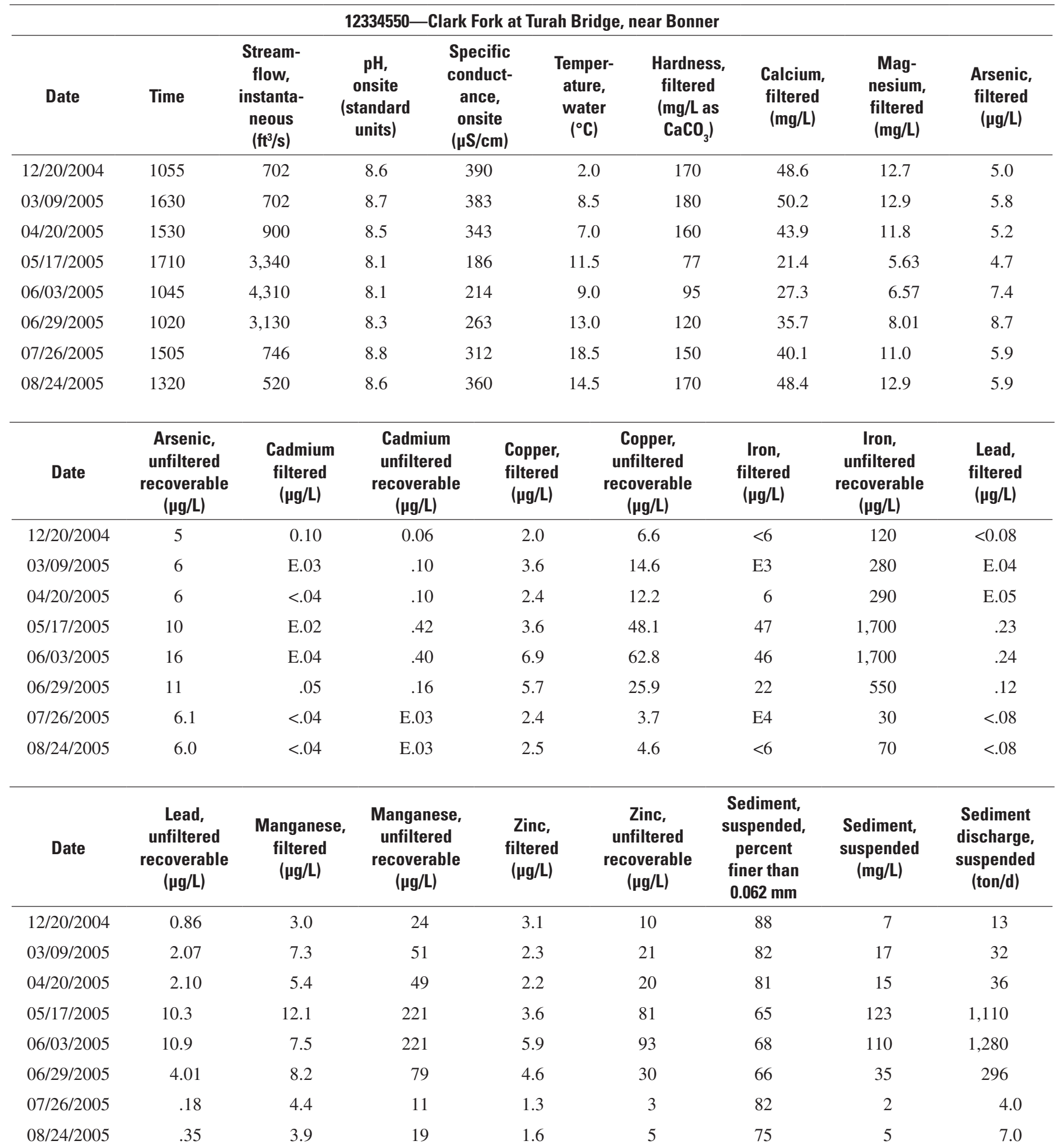


Table 4. Water-quality data for the upper Clark Fork basin, Montana, October 2004 through September 2005.-Continued

[Abbreviations: $\mathrm{ft} \mathrm{t}^{3} / \mathrm{s}$, cubic feet per second; ${ }^{\circ} \mathrm{C}$, degrees Celsius; E, estimated; $\mu \mathrm{g} / \mathrm{L}$, micrograms per liter; $\mu \mathrm{S} / \mathrm{cm}$, microsiemens per centimeter at $25^{\circ} \mathrm{C}$; $\mathrm{mg} / \mathrm{L}$, milligrams per liter; mm, millimeters; ton/d, tons per day. Symbol: $<$, less than laboratory reporting level]

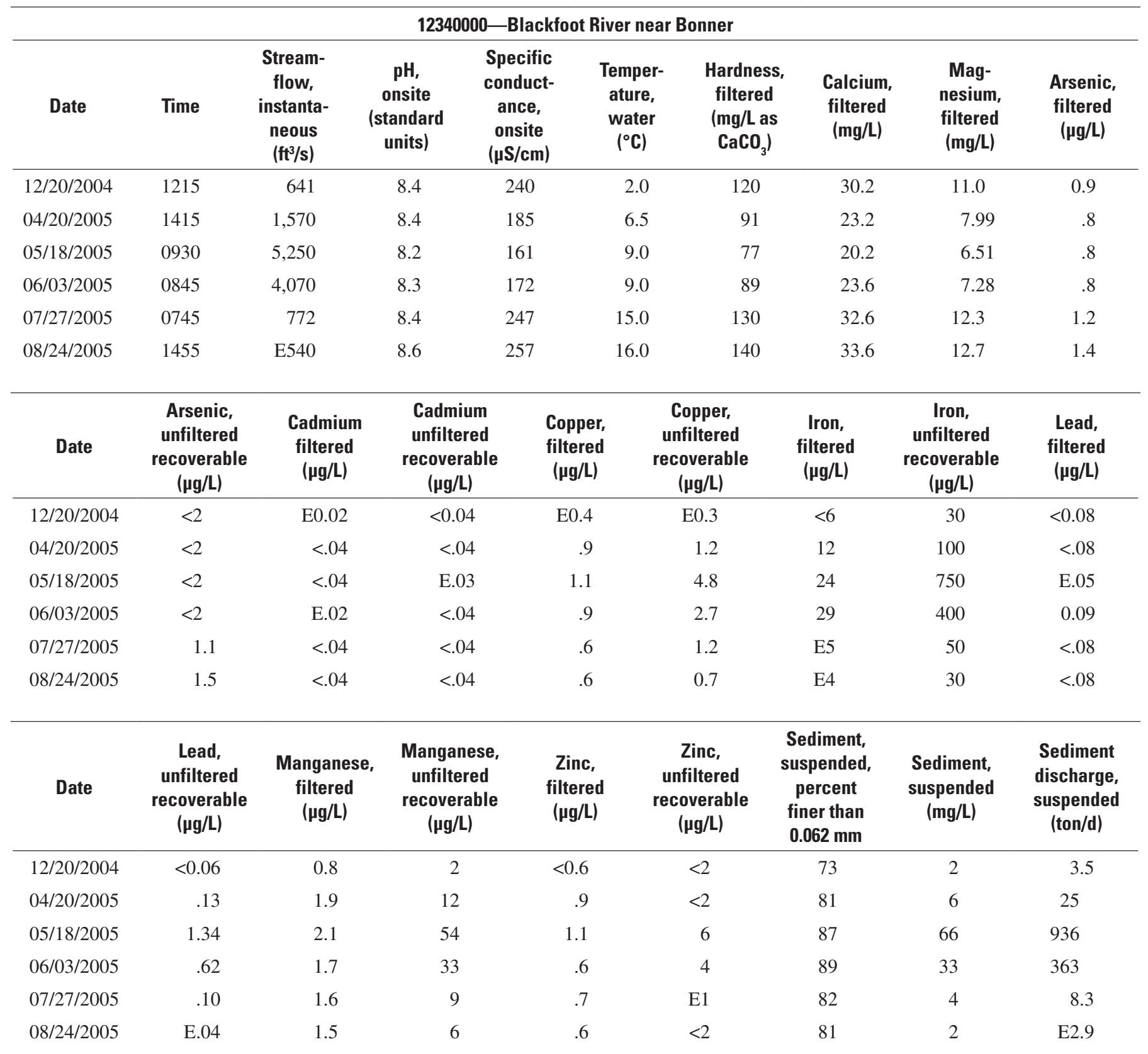


Table 4. Water-quality data for the upper Clark Fork basin, Montana, October 2004 through September 2005.-Continued

[Abbreviations: $\mathrm{ft} 3 / \mathrm{s}$, cubic feet per second; ${ }^{\circ} \mathrm{C}$, degrees Celsius; E, estimated; $\mu \mathrm{g} / \mathrm{L}$, micrograms per liter; $\mu \mathrm{S} / \mathrm{cm}$, microsiemens per centimeter at $25^{\circ} \mathrm{C}$; $\mathrm{mg} / \mathrm{L}$, milligrams per liter; mm, millimeters; ton/d, tons per day. Symbol: $<$, less than laboratory reporting level]

\begin{tabular}{|c|c|c|c|c|c|c|c|c|c|c|}
\hline \multicolumn{11}{|c|}{ 12340500—Clark Fork above Missoula } \\
\hline Date & Time & $\begin{array}{c}\text { Stream- } \\
\text { flow, } \\
\text { instanta- } \\
\text { neous } \\
\left(\mathrm{ft}^{3} / \mathrm{s}\right)\end{array}$ & $\begin{array}{c}\text { pH, } \\
\text { onsite } \\
\text { (standard } \\
\text { units) }\end{array}$ & $\begin{array}{c}\text { Specific } \\
\text { conduct- } \\
\text { ance, } \\
\text { onsite } \\
(\mu \mathrm{S} / \mathrm{cm})\end{array}$ & & $\begin{array}{l}\text { Temper- } \\
\text { ature, } \\
\text { water } \\
\left({ }^{\circ} \mathrm{C}\right)\end{array}$ & $\begin{array}{c}\text { Hardness, } \\
\text { filtered } \\
\text { (mg/L as } \\
\left.\mathrm{CaCO}_{3}\right)\end{array}$ & $\begin{array}{l}\text { Calcium, } \\
\text { filtered } \\
\text { (mg/L) }\end{array}$ & $\begin{array}{l}\text { Mag- } \\
\text { nesium, } \\
\text { filtered } \\
(\mathrm{mg} / \mathrm{L})\end{array}$ & $\begin{array}{c}\text { Arsenic, } \\
\text { filtered } \\
\text { ( } \mu \mathrm{g} / \mathrm{L})\end{array}$ \\
\hline $12 / 20 / 2004$ & 1350 & 1,290 & 8.6 & 323 & & 3.0 & 150 & 40.5 & 12.2 & 3.2 \\
\hline 03/10/2005 & 0750 & 1,280 & 8.4 & 312 & & 7.0 & 150 & 40.3 & 11.8 & 3.6 \\
\hline 04/20/2005 & 1030 & 2,360 & 8.4 & 248 & & 7.0 & 120 & 31.2 & 9.30 & 2.5 \\
\hline 05/18/2005 & 0715 & 9,110 & 8.1 & 170 & & 9.5 & 77 & 20.7 & 6.11 & 2.7 \\
\hline 06/03/2005 & 0645 & 7,960 & 8.2 & 193 & & 9.0 & 91 & 25.1 & 6.79 & 4.0 \\
\hline 06/29/2005 & 1235 & 5,180 & 8.3 & 243 & & 13.5 & 120 & 32.9 & 8.39 & 5.4 \\
\hline $07 / 27 / 2005$ & 0900 & 1,430 & 8.5 & 276 & & 18.0 & 140 & 37.0 & 11.6 & 3.8 \\
\hline 08/24/2005 & 1630 & 959 & 8.5 & 306 & & 16.0 & 150 & 39.4 & 12.3 & 3.7 \\
\hline Date & $\begin{array}{c}\text { Arsenic, } \\
\text { unfiltered } \\
\text { recoverable } \\
(\mu \mathrm{g} / \mathrm{L})\end{array}$ & $\begin{array}{c}\text { Cadmium } \\
\text { filtered } \\
(\mu \mathrm{g} / \mathrm{L})\end{array}$ & $\begin{array}{r}\text { Cadn } \\
\text { unfil } \\
\text { recov } \\
(\mu g \\
\end{array}$ & & $\begin{array}{l}\text { Copper, } \\
\text { filtered } \\
(\mu \mathrm{g} / \mathrm{L})\end{array}$ & & $\begin{array}{c}\text { Copper, } \\
\text { unfiltered } \\
\text { ecoverable } \\
\text { ( } \mu \mathrm{g} / \mathrm{L})\end{array}$ & $\begin{array}{l}\text { Iron, } \\
\text { filtered } \\
(\mu \mathrm{g} / \mathrm{L})\end{array}$ & $\begin{array}{c}\text { Iron, } \\
\text { unfiltered } \\
\text { recoverable } \\
(\mu \mathrm{g} / \mathrm{L})\end{array}$ & $\begin{array}{c}\text { Lead, } \\
\text { filtered } \\
(\mu \mathrm{g} / \mathrm{L})\end{array}$ \\
\hline $12 / 20 / 2004$ & 3 & E0.03 & 0. & & 1.4 & & 3.3 & $<6$ & 70 & $<0.08$ \\
\hline 03/10/2005 & 3 & $<.04$ & & & 2.1 & & 6.5 & 7 & 120 & E.05 \\
\hline $04 / 20 / 2005$ & 2 & $<.04$ & & & 1.4 & & 5.5 & 13 & 150 & E. 05 \\
\hline 05/18/2005 & 6 & $<.04$ & & & 2.3 & & 26.2 & 43 & 1,260 & .16 \\
\hline $06 / 03 / 2005$ & 7 & E.03 & & & 4.2 & & 31.9 & 32 & 930 & .17 \\
\hline $06 / 29 / 2005$ & 6 & E. 03 & & & 4.3 & & 14.8 & 16 & 330 & .09 \\
\hline $07 / 27 / 2005$ & 4.0 & $<.04$ & & & 2.4 & & 6.1 & 9 & 80 & E. 05 \\
\hline $08 / 24 / 2005$ & 3.9 & $<.04$ & E. & & 1.8 & & 4.2 & 7 & 80 & E.04 \\
\hline Date & $\begin{array}{c}\text { Lead, } \\
\text { unfiltered } \\
\text { recoverable } \\
(\mu \mathrm{g} / \mathrm{L})\end{array}$ & $\begin{array}{c}\text { Manganese, } \\
\text { filtered } \\
(\mu \mathrm{g} / \mathrm{L})\end{array}$ & $\begin{array}{r}\text { Mang } \\
\text { unfil } \\
\text { recov } \\
(\mu g\end{array}$ & & $\begin{array}{l}\text { Zinc, } \\
\text { filtered } \\
(\mu \mathrm{g} / \mathrm{L})\end{array}$ & & $\begin{array}{c}\text { Zinc, } \\
\text { unfiltered } \\
\text { ecoverable } \\
\text { ( } \mu \mathrm{g} / \mathrm{L})\end{array}$ & $\begin{array}{c}\text { Sediment, } \\
\text { suspended, } \\
\text { percent } \\
\text { finer than } \\
0.062 \mathrm{~mm}\end{array}$ & $\begin{array}{l}\text { Sediment, } \\
\text { suspended } \\
(\mathrm{mg} / \mathrm{L})\end{array}$ & $\begin{array}{c}\text { Sediment } \\
\text { discharge, } \\
\text { suspended } \\
\text { (ton/d) }\end{array}$ \\
\hline $12 / 20 / 2004$ & 0.43 & 7.5 & & & 2.3 & & 6 & 86 & 5 & 17 \\
\hline 03/10/2005 & .73 & 30.3 & & & 6.3 & & 9 & 90 & 7 & 24 \\
\hline $04 / 20 / 2005$ & .83 & 14.1 & & & 1.5 & & 9 & 92 & 9 & 57 \\
\hline 05/18/2005 & 5.91 & 8.8 & 12 & & 2.4 & & 44 & 90 & 89 & 2,190 \\
\hline 06/03/2005 & 5.32 & 8.0 & 12 & & 3.9 & & 48 & 90 & 55 & 1,180 \\
\hline $06 / 29 / 2005$ & 2.23 & 13.6 & & & 3.8 & & 19 & 89 & 18 & 252 \\
\hline $07 / 27 / 2005$ & .52 & 13.8 & & & 1.3 & & 8 & 92 & 5 & 19 \\
\hline $08 / 24 / 2005$ & .46 & 14.1 & & & 1.2 & & 5 & 84 & 5 & 13 \\
\hline
\end{tabular}


Table 5. Daily mean streamflow and suspended-sediment data for Clark Fork at Deer Lodge, Montana, October 2004 through September 2005.

[Abbreviations: acre-ft, acre-feet; $\mathrm{ft}^{3} / \mathrm{s}$, cubic feet per second; e, estimated; max, maximum; milligrams per liter; min, minimum; mg/L, ton/d, tons per day. Symbol: ---, no data or value not computed]

\begin{tabular}{|c|c|c|c|c|c|c|c|c|c|}
\hline \multirow{3}{*}{ Day } & \multirow{2}{*}{$\begin{array}{c}\text { Mean } \\
\text { stream- } \\
\text { flow } \\
\left(\mathrm{ft}^{3} / \mathrm{s}\right)\end{array}$} & \multicolumn{2}{|c|}{ Suspended sediment } & \multirow{2}{*}{$\begin{array}{c}\text { Mean } \\
\text { stream- } \\
\text { flow } \\
\left(\mathrm{ft}^{3} / \mathrm{s}\right)\end{array}$} & \multicolumn{2}{|c|}{ Suspended sediment } & \multirow{2}{*}{$\begin{array}{c}\text { Mean } \\
\text { stream- } \\
\text { flow } \\
\left(\mathrm{ft}^{3} / \mathrm{s}\right)\end{array}$} & \multicolumn{2}{|c|}{ Suspended sediment } \\
\hline & & $\begin{array}{c}\text { Mean } \\
\text { concentration } \\
\text { (mg/L) }\end{array}$ & $\begin{array}{c}\text { Discharge } \\
\text { (ton/d) }\end{array}$ & & $\begin{array}{c}\text { Mean } \\
\text { concentration } \\
\text { (mg/L) }\end{array}$ & $\begin{array}{c}\text { Discharge } \\
\text { (ton/d) }\end{array}$ & & $\begin{array}{c}\text { Mean } \\
\text { concentration } \\
(\mathrm{mg} / \mathrm{L})\end{array}$ & $\begin{array}{c}\text { Discharge } \\
\text { (ton/d) }\end{array}$ \\
\hline & \multicolumn{3}{|c|}{ October } & \multicolumn{3}{|c|}{ November } & \multicolumn{3}{|c|}{ December } \\
\hline 1 & 126 & 11 & 3.7 & 154 & 15 & 6.2 & e140 & 20 & 7.6 \\
\hline 4 & 132 & 10 & 3.6 & 168 & 19 & 8.6 & 172 & 17 & 7.9 \\
\hline 5 & 133 & 10 & 3.6 & 165 & 16 & 7.1 & 175 & 16 & 7.6 \\
\hline 6 & 132 & 10 & 3.6 & 172 & 13 & 6.0 & 179 & 15 & 7.2 \\
\hline 7 & 130 & 11 & 3.9 & 168 & 11 & 5.0 & 178 & 15 & 7.2 \\
\hline 11 & 152 & 14 & 5.7 & 170 & 14 & 6.4 & 195 & 19 & 10 \\
\hline 12 & 163 & 17 & 7.5 & 168 & 14 & 6.4 & 196 & 20 & 11 \\
\hline 13 & 164 & 18 & 8.0 & 165 & 14 & 6.2 & 183 & 20 & 9.9 \\
\hline 14 & 171 & 18 & 8.3 & 166 & 15 & 6.7 & 189 & 19 & 9.7 \\
\hline 15 & 169 & 15 & 6.8 & 168 & 15 & 6.8 & 189 & 18 & 9.2 \\
\hline 16 & 165 & 13 & 5.8 & 177 & 18 & 8.6 & 185 & 17 & 8.5 \\
\hline 17 & 160 & 12 & 5.2 & 173 & 21 & 9.8 & 185 & 16 & 8.0 \\
\hline 18 & 166 & 12 & 5.4 & 173 & 22 & 10 & 183 & 17 & 8.4 \\
\hline 24 & 162 & 12 & 5.2 & 200 & 23 & 12 & e160 & 21 & 9.1 \\
\hline 25 & 158 & 11 & 4.7 & 214 & 26 & 15 & 174 & 22 & 10 \\
\hline 26 & 154 & 11 & 4.6 & 222 & 26 & 16 & e180 & 24 & 12 \\
\hline 27 & 156 & 12 & 5.1 & 202 & 26 & 14 & e180 & 28 & 14 \\
\hline 28 & 163 & 12 & 5.3 & e190 & 24 & 12 & e170 & 30 & 14 \\
\hline 29 & 168 & 13 & 5.9 & e170 & 22 & 10 & e170 & 27 & 12 \\
\hline 30 & 164 & 13 & 5.8 & e150 & 20 & 8.1 & 176 & 23 & 11 \\
\hline 31 & 158 & 13 & 5.5 & --- & --- & --- & e150 & 20 & 8.1 \\
\hline Total & 4,751 & --. & 163.4 & 5,253 & --. & 263.7 & 5,413 & --. & 284.1 \\
\hline Mean & 153 & 13 & 5.3 & 175 & 18 & 8.8 & 175 & 19 & 9.2 \\
\hline Max & 175 & 18 & 8.3 & 222 & 26 & 16 & 196 & 30 & 14 \\
\hline Min & 126 & 10 & 3.6 & 150 & 11 & 4.9 & 130 & 14 & 6.7 \\
\hline
\end{tabular}


Table 5. Daily mean streamflow and suspended-sediment data for Clark Fork at Deer Lodge, Montana, October 2004 through September 2005.-Continued

[Abbreviations: acre-ft, acre-feet; $\mathrm{ft}^{3} / \mathrm{s}$, cubic feet per second; e, estimated; max, maximum; mg/L, milligrams per liter; min, minimum; ton/d, tons per day. Symbol: ---, no data or value not computed]

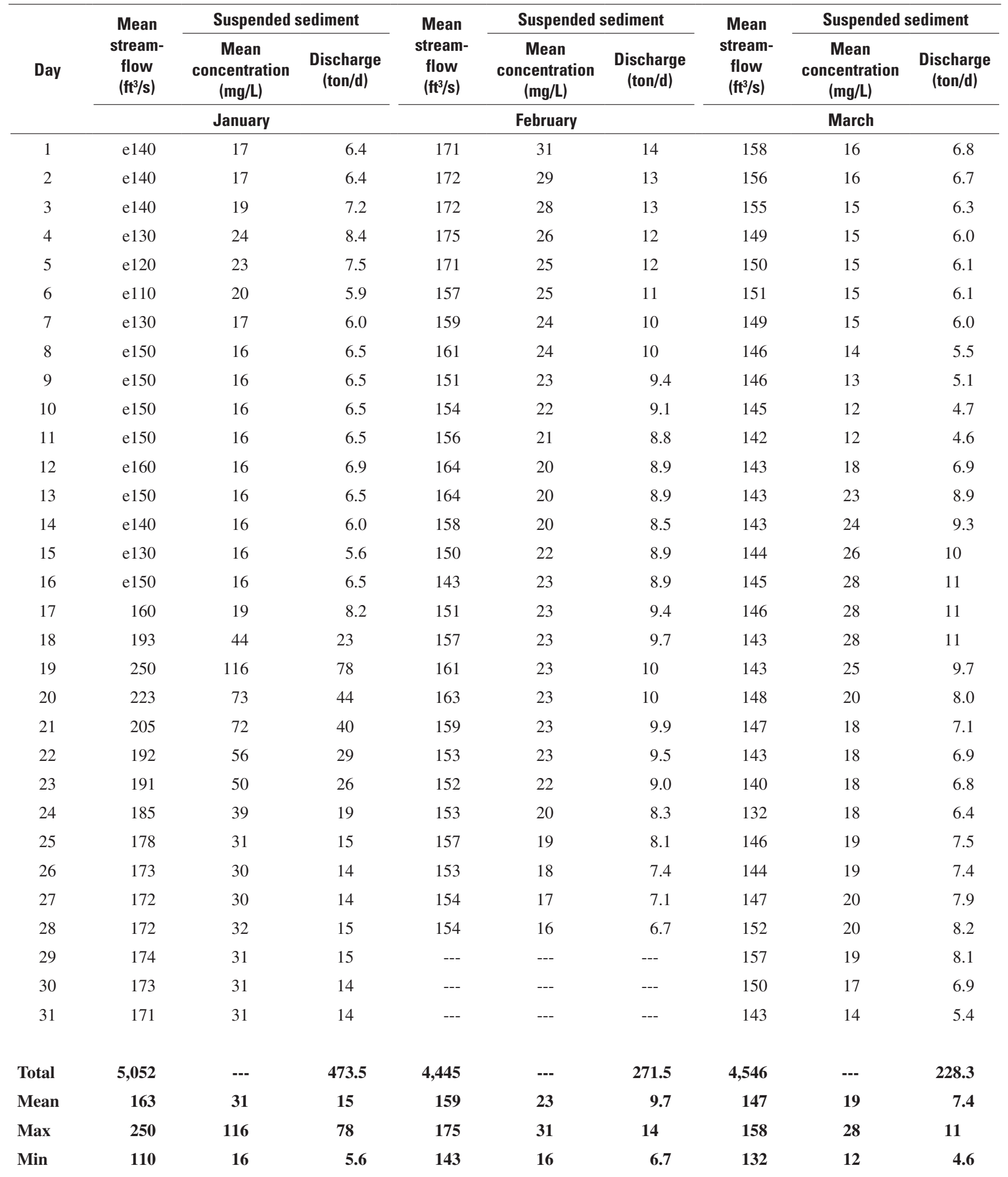


Table 5. Daily mean streamflow and suspended-sediment data for Clark Fork at Deer Lodge, Montana, October 2004 through September 2005.-Continued

[Abbreviations: acre-ft, acre-feet; $\mathrm{ft}^{3} / \mathrm{s}$, cubic feet per second; e, estimated; max, maximum; mg/L, milligrams per liter; min, minimum; ton/d, tons per day. Symbol: ---, no data or value not computed]

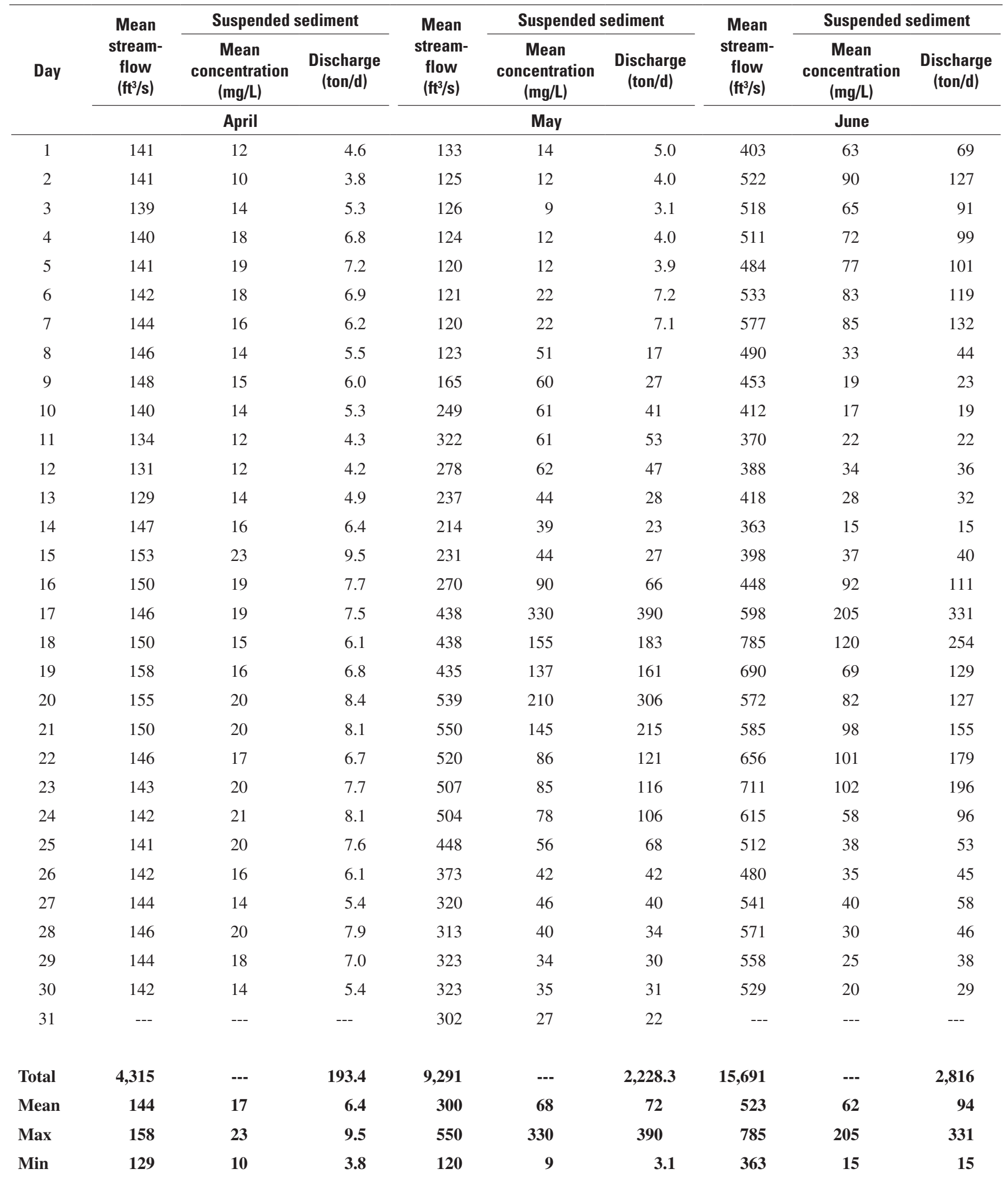


Table 5. Daily mean streamflow and suspended-sediment data for Clark Fork at Deer Lodge, Montana, October 2004 through September 2005.-Continued

[Abbreviations: acre-ft, acre-feet; $\mathrm{ft}^{3} / \mathrm{s}$, cubic feet per second; e, estimated; max, maximum; mg/L, milligrams per liter; min, minimum; ton/d, tons per day. Symbol: ---, no data or value not computed]

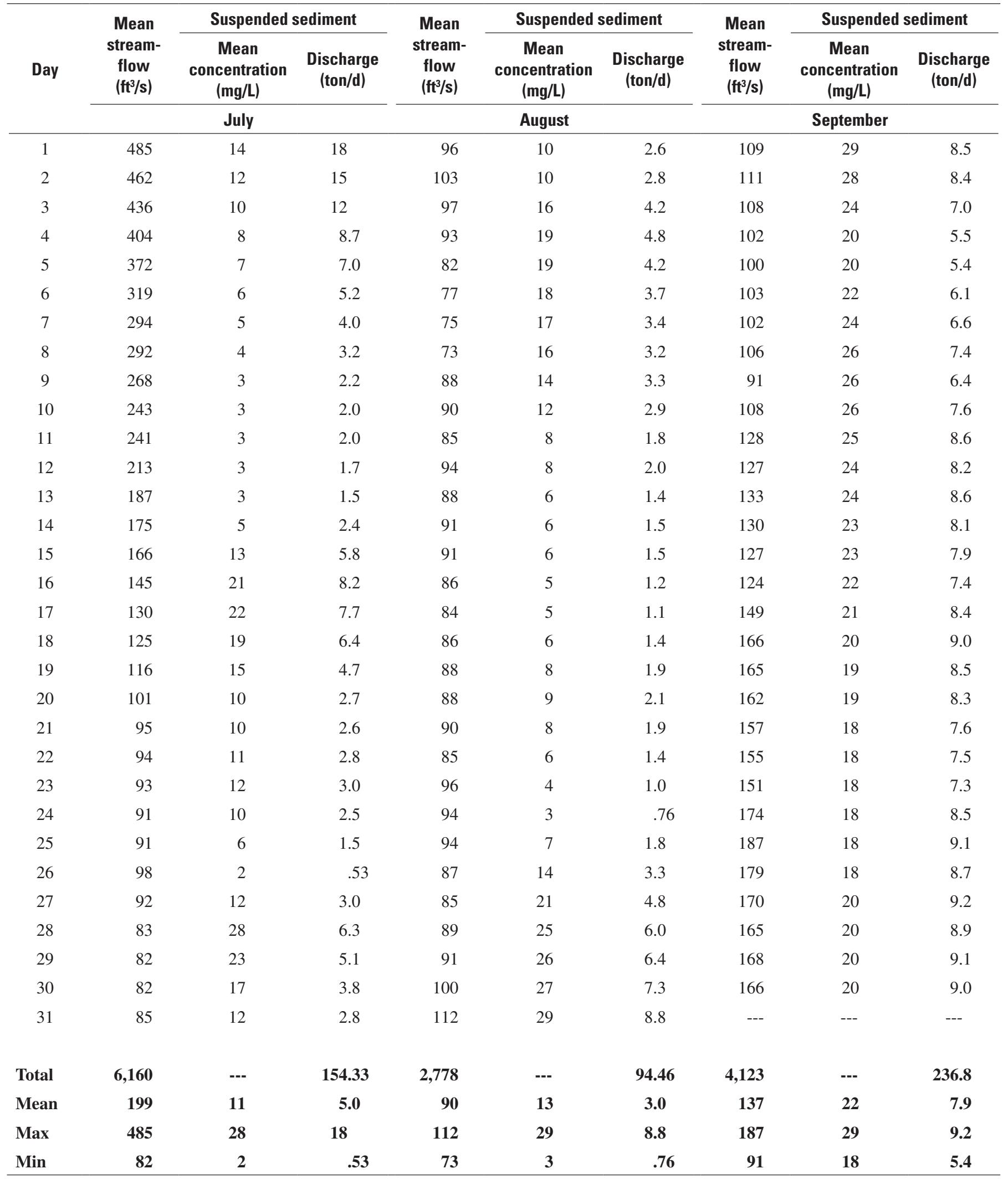

Total for water year 2005 (unrounded sum of the daily values): streamflow, 71,818 ft³/s (142,500 acre-ft); sediment load, 7,407.79 tons. 
Table 6. Daily mean streamflow and suspended-sediment data for Clark Fork at Turah Bridge, near Bonner, Montana, 0 ctober 2004 through September 2005.

[Abbreviations: acre-ft, acre-feet; $\mathrm{ft}^{3} / \mathrm{s}$, cubic feet per second; e, estimated; max, maximum; mg/L, milligrams per liter; min, minimum; ton/d, tons per day. Symbol: ---, no data or value not computed]

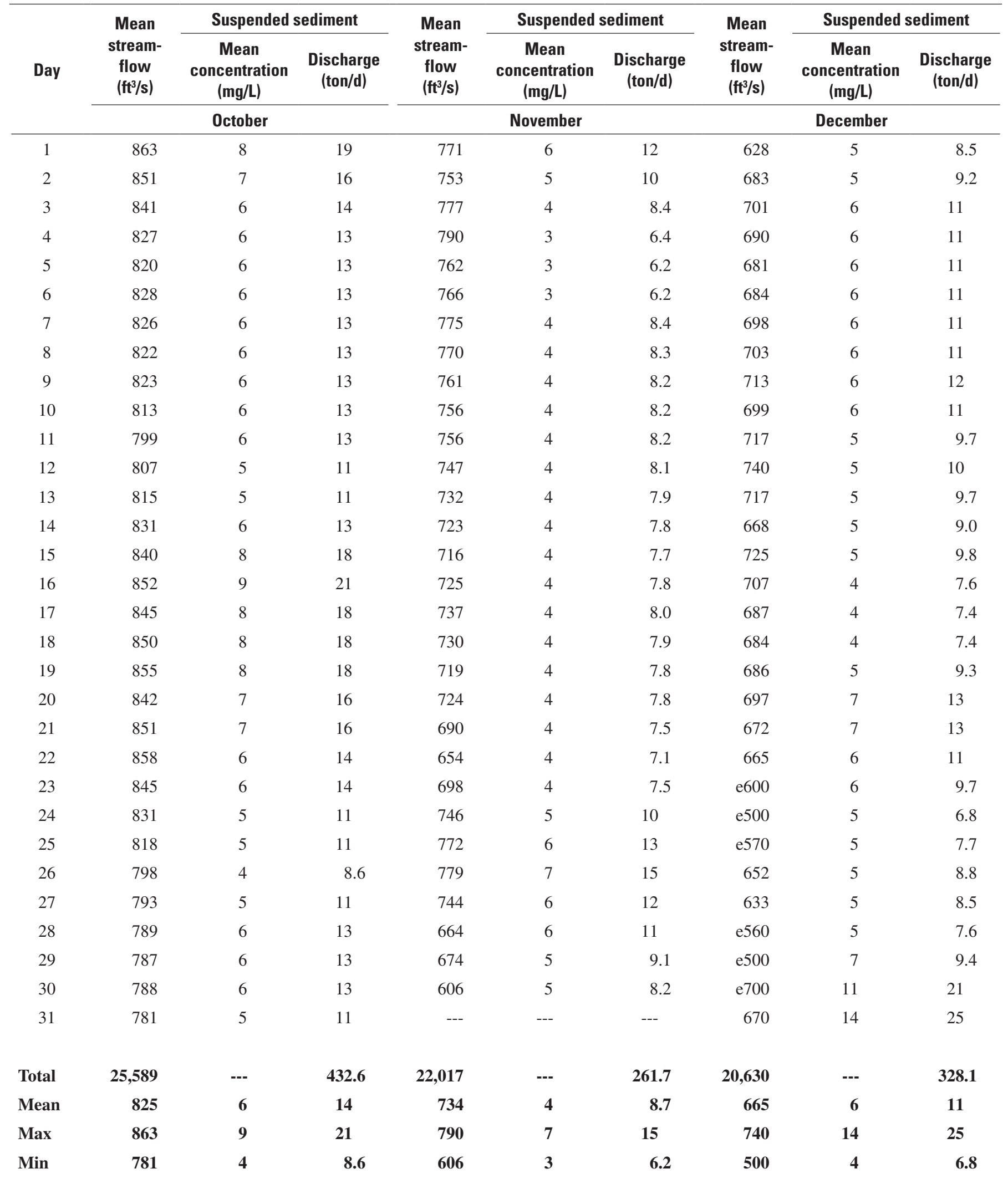


Table 6. Daily mean streamflow and suspended-sediment data for Clark Fork at Turah Bridge, near Bonner, Montana, October 2004 through September 2005.-Continued

[Abbreviations: acre-ft, acre-feet; $\mathrm{ft}^{3} / \mathrm{s}$, cubic feet per second; e, estimated; max, maximum; $\mathrm{mg} / \mathrm{L}$, milligrams per liter; min, minimum; ton/d, tons per day. Symbol: ---, no data or value not computed]

\begin{tabular}{|c|c|c|c|c|c|c|c|c|c|}
\hline \multirow{3}{*}{ Day } & \multirow{2}{*}{$\begin{array}{c}\text { Mean } \\
\text { stream- } \\
\text { flow } \\
\left(\mathrm{ft}^{3} / \mathrm{s}\right)\end{array}$} & \multicolumn{2}{|c|}{ Suspended sediment } & \multirow{2}{*}{$\begin{array}{c}\text { Mean } \\
\text { stream- } \\
\text { flow } \\
\left(\mathrm{ft}^{3} / \mathrm{s}\right)\end{array}$} & \multicolumn{2}{|c|}{ Suspended sediment } & \multirow{2}{*}{$\begin{array}{c}\text { Mean } \\
\text { stream- } \\
\text { flow } \\
\left(\mathrm{ft}^{3} / \mathrm{s}\right)\end{array}$} & \multicolumn{2}{|c|}{ Suspended sediment } \\
\hline & & $\begin{array}{c}\text { Mean } \\
\text { concentration } \\
(\mathrm{mg} / \mathrm{L})\end{array}$ & $\begin{array}{c}\text { Discharge } \\
\text { (ton/d) }\end{array}$ & & $\begin{array}{c}\text { Mean } \\
\text { concentration } \\
(\mathrm{mg} / \mathrm{L})\end{array}$ & $\begin{array}{c}\text { Discharge } \\
\text { (ton/d) }\end{array}$ & & $\begin{array}{c}\text { Mean } \\
\text { concentration } \\
(\mathrm{mg} / \mathrm{L})\end{array}$ & $\begin{array}{c}\text { Discharge } \\
\text { (ton/d) }\end{array}$ \\
\hline & \multicolumn{3}{|c|}{ January } & \multicolumn{3}{|c|}{ February } & \multicolumn{3}{|c|}{ March } \\
\hline 1 & 591 & 14 & 22 & 707 & 10 & 19 & 607 & 9 & 15 \\
\hline 4 & e 270 & 4 & 2.9 & 690 & 12 & 22 & 634 & 13 & 22 \\
\hline 5 & e220 & 4 & 2.4 & 696 & 13 & 24 & 636 & 14 & 24 \\
\hline 6 & e230 & 4 & 2.5 & 674 & 13 & 24 & 648 & 14 & 24 \\
\hline 7 & e280 & 4 & 3.0 & 619 & 13 & 22 & 669 & 16 & 29 \\
\hline 11 & e440 & 4 & 4.8 & 587 & 12 & 19 & 708 & 21 & 40 \\
\hline 12 & e 480 & 4 & 5.2 & 607 & 11 & 18 & 707 & 22 & 42 \\
\hline 13 & e540 & 4 & 5.8 & 646 & 11 & 19 & 707 & 19 & 36 \\
\hline 14 & e560 & 5 & 7.6 & 637 & 10 & 17 & 666 & 17 & 31 \\
\hline 15 & e500 & 5 & 6.8 & 609 & 10 & 16 & 660 & 14 & 25 \\
\hline 16 & e400 & 5 & 5.4 & 572 & 10 & 15 & 655 & 14 & 25 \\
\hline 17 & e460 & 6 & 7.5 & 528 & 10 & 14 & 666 & 14 & 25 \\
\hline 18 & e550 & 13 & 19 & 527 & 9 & 13 & 656 & 12 & 21 \\
\hline 24 & 819 & 10 & 22 & 590 & 7 & 11 & 618 & 9 & 15 \\
\hline 25 & 778 & 9 & 19 & 601 & 6 & 9.7 & 604 & 9 & 15 \\
\hline 26 & 759 & 8 & 16 & 609 & 6 & 9.9 & 622 & 11 & 18 \\
\hline 27 & 746 & 8 & 16 & 604 & 6 & 9.8 & 615 & 14 & 23 \\
\hline 28 & 746 & 8 & 16 & 600 & 7 & 11 & 663 & 18 & 32 \\
\hline 29 & 744 & 10 & 20 & --- & --- & --- & 735 & 23 & 46 \\
\hline 30 & 740 & 10 & 20 & --- & --- & --- & 741 & 21 & 42 \\
\hline 31 & 722 & 10 & 19 & --- & --- & --- & 700 & 14 & 26 \\
\hline Total & 17,921 & --- & 612.4 & 17,324 & --- & 451.4 & 20,524 & --- & 845 \\
\hline Mean & 578 & 10 & 20 & 619 & 10 & 16 & 662 & 15 & 27 \\
\hline Max & 968 & 42 & 110 & 707 & 13 & 24 & 741 & 23 & 46 \\
\hline Min & 220 & 4 & 2.4 & 527 & 6 & 9.7 & 604 & 9 & 15 \\
\hline
\end{tabular}


Table 6. Daily mean streamflow and suspended-sediment data for Clark Fork at Turah Bridge, near Bonner, Montana, October 2004 through September 2005.-Continued

[Abbreviations: acre-ft, acre-feet; $\mathrm{ft}^{3} / \mathrm{s}$, cubic feet per second; e, estimated; max, maximum; $\mathrm{mg} / \mathrm{L}$, milligrams per liter; min, minimum; ton/d, tons per day. Symbol: ---, no data or value not computed]

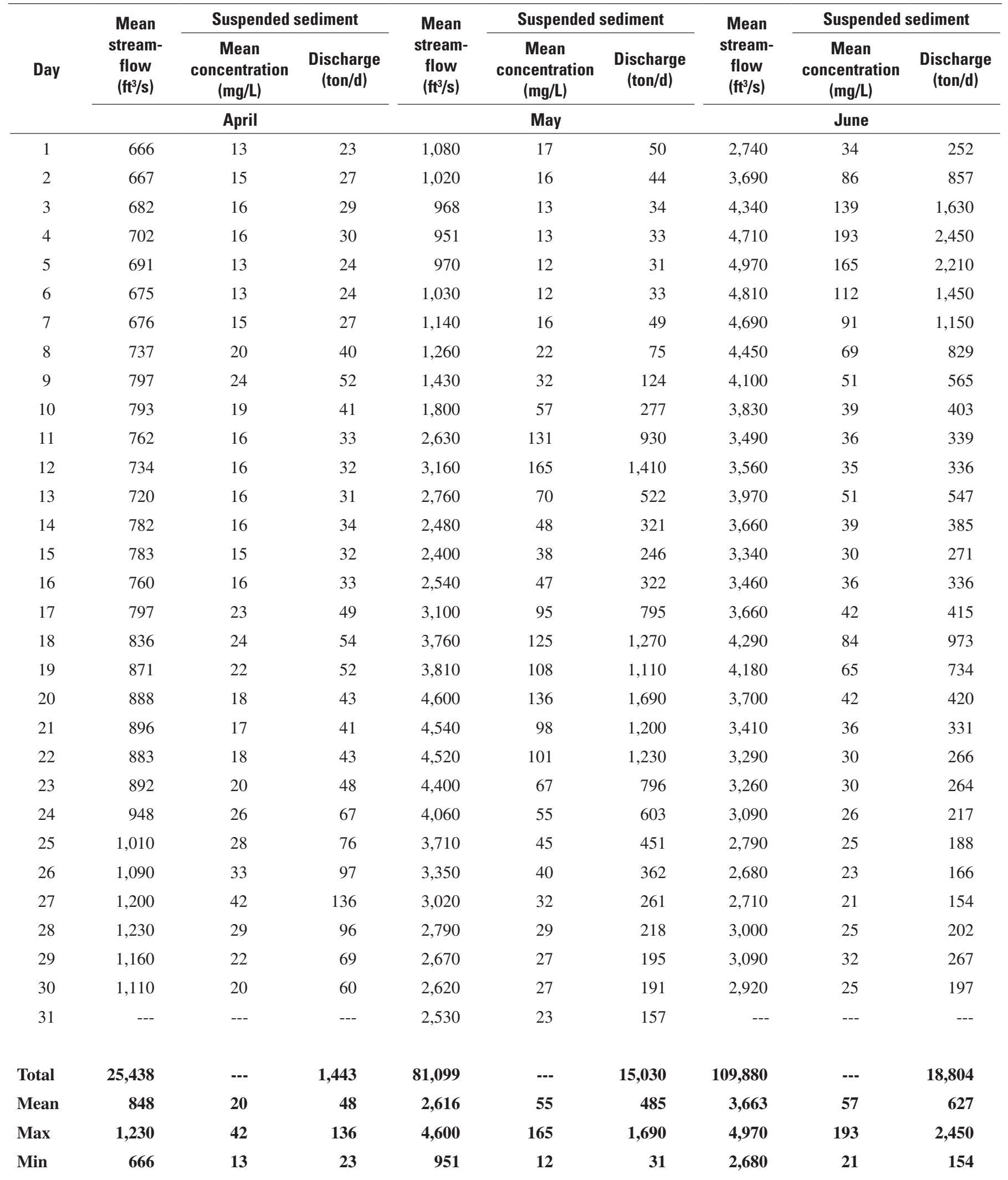


Table 6. Daily mean streamflow and suspended-sediment data for Clark Fork at Turah Bridge, near Bonner, Montana, October 2004 through September 2005.-Continued

[Abbreviations: acre-ft, acre-feet; $\mathrm{ft}^{3} / \mathrm{s}$, cubic feet per second; e, estimated; max, maximum; mg/L, milligrams per liter; min, minimum; ton/d, tons per day. Symbol: ---, no data or value not computed]

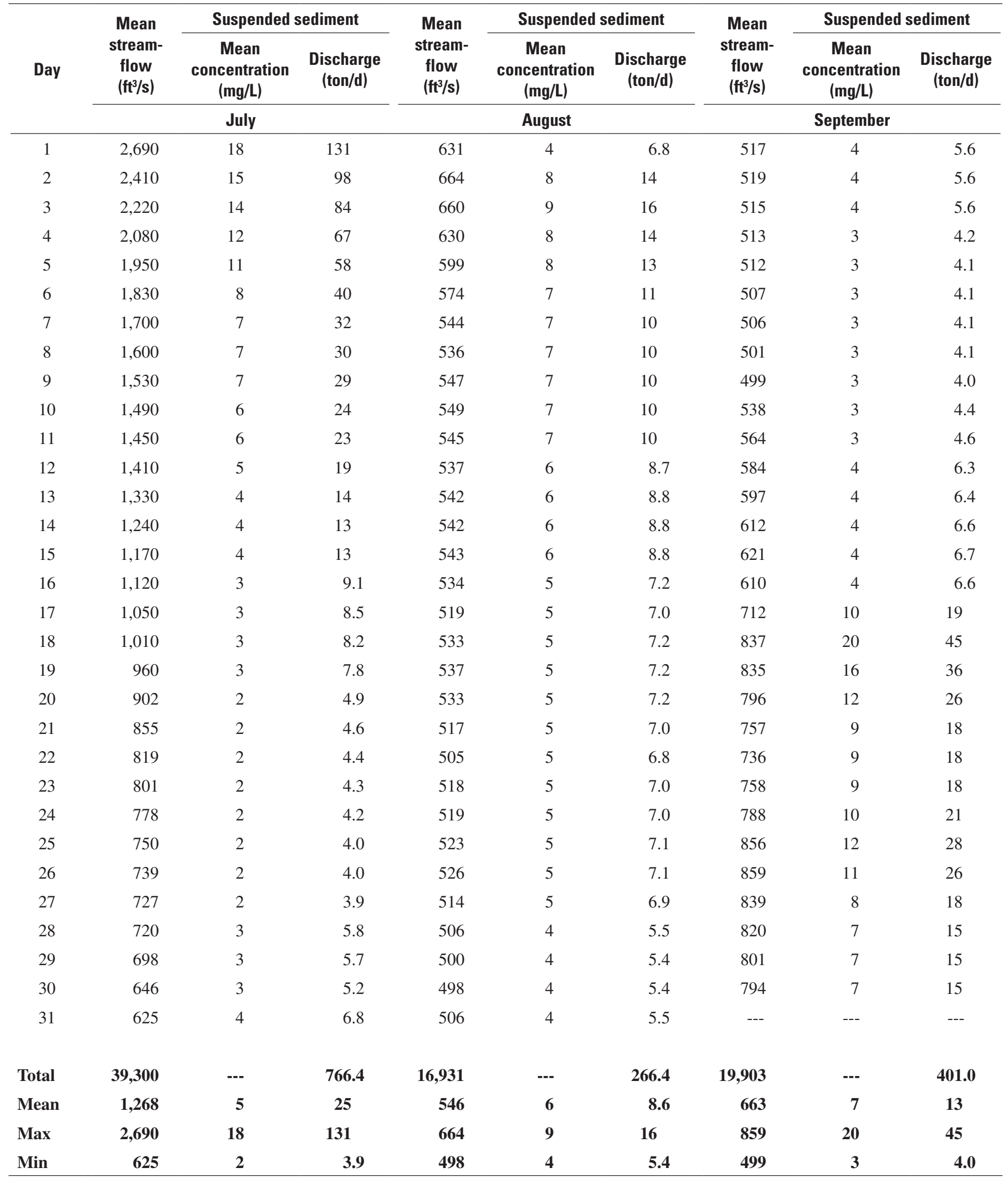

Total for water year 2005 (unrounded sum of daily values): streamflow, 416,556 ft³/s (826,200 acre-ft); sediment load, 39,642 tons. 
Table 7. Daily mean streamflow and suspended-sediment data for Clark Fork above Missoula, Montana, October 2004 through September 2005.

[Abbreviations: acre-ft, acre-feet; $\mathrm{ft}^{3} / \mathrm{s}$, cubic feet per second; e, estimated; max, maximum; mg/L, milligrams per liter; min, minimum; ton/d, tons per day. Symbol: ---, no data or value not computed]

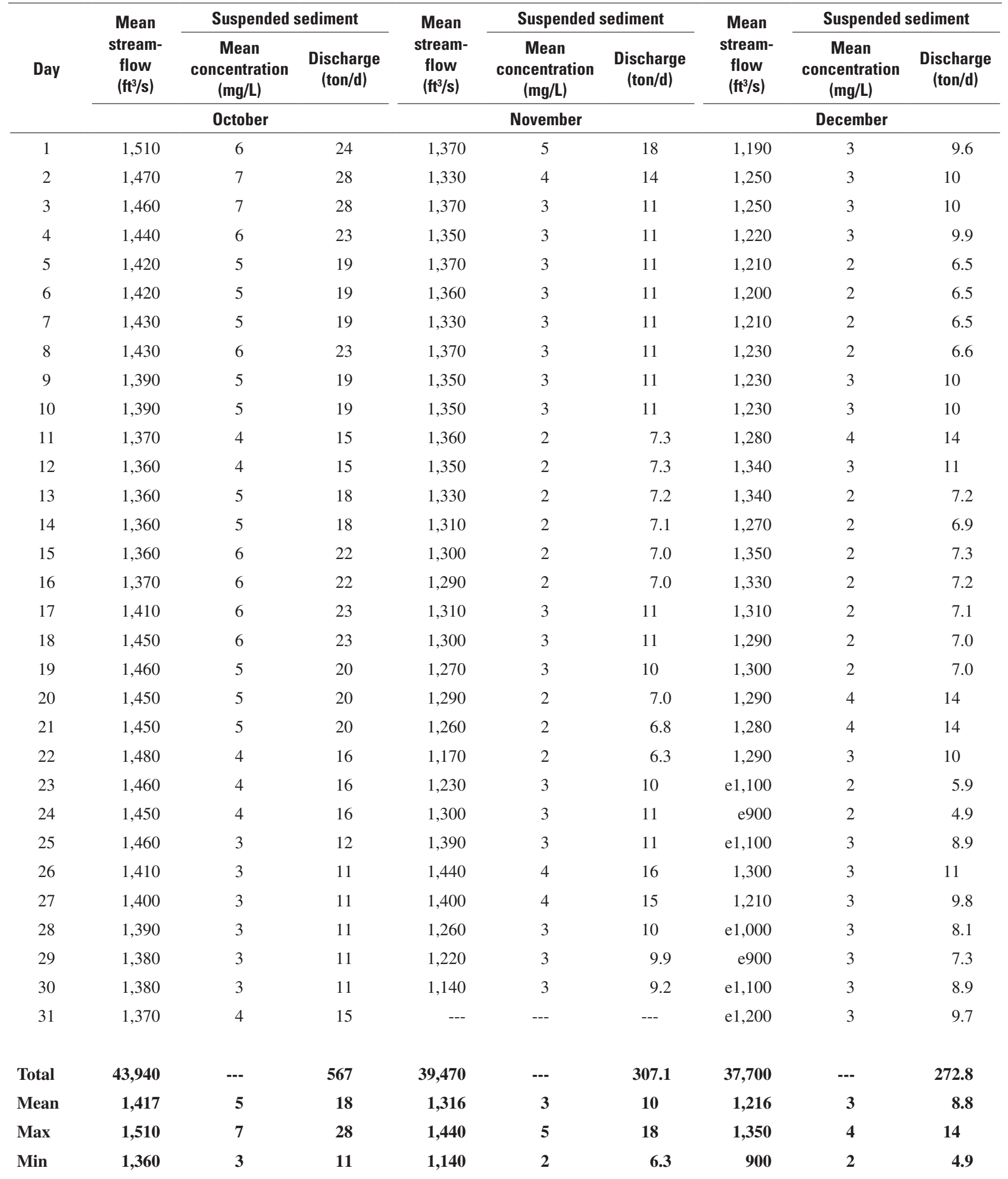


Table 7. Daily mean streamflow and suspended-sediment data for Clark Fork above Missoula, Montana, October 2004 through September 2005.-Continued

[Abbreviations: acre-ft, acre-feet; $\mathrm{ft}^{3} / \mathrm{s}$, cubic feet per second; e, estimated; max, maximum; mg/L, milligrams per liter; min, minimum; ton/d, tons per day. Symbol: ---, no data or value not computed]

\begin{tabular}{|c|c|c|c|c|c|c|c|c|c|}
\hline \multirow{3}{*}{ Day } & \multirow{2}{*}{$\begin{array}{c}\text { Mean } \\
\text { stream- } \\
\text { flow } \\
\left(\mathrm{ft}^{3} / \mathrm{s}\right)\end{array}$} & \multicolumn{2}{|c|}{ Suspended sediment } & \multirow{2}{*}{$\begin{array}{c}\text { Mean } \\
\text { stream- } \\
\text { flow } \\
\left(\mathrm{ft}^{3} / \mathrm{s}\right)\end{array}$} & \multicolumn{2}{|c|}{ Suspended sediment } & \multirow{2}{*}{$\begin{array}{c}\text { Mean } \\
\text { stream- } \\
\text { flow } \\
\left(\mathrm{ft}^{3} / \mathrm{s}\right)\end{array}$} & \multicolumn{2}{|c|}{ Suspended sediment } \\
\hline & & $\begin{array}{c}\text { Mean } \\
\text { concentration } \\
(\mathrm{mg} / \mathrm{L})\end{array}$ & $\begin{array}{l}\text { Discharge } \\
\text { (ton/d) }\end{array}$ & & $\begin{array}{c}\text { Mean } \\
\text { concentration } \\
\text { (mg/L) }\end{array}$ & $\begin{array}{l}\text { Discharge } \\
\text { (ton/d) }\end{array}$ & & $\begin{array}{c}\text { Mean } \\
\text { concentration } \\
\text { (mg/L) }\end{array}$ & $\begin{array}{c}\text { Discharge } \\
\text { (ton/d) }\end{array}$ \\
\hline & \multicolumn{3}{|c|}{ January } & \multicolumn{3}{|c|}{ February } & \multicolumn{3}{|c|}{ March } \\
\hline 1 & e950 & 3 & 7.7 & 1,450 & 6 & 23 & 1,100 & 5 & 15 \\
\hline 4 & e530 & 3 & 4.3 & 1,380 & 5 & 19 & 1,130 & 5 & 15 \\
\hline 5 & e500 & 3 & 4.0 & 1,390 & 5 & 19 & 1,150 & 5 & 16 \\
\hline 6 & e550 & 3 & 4.5 & 1,370 & 4 & 15 & 1,160 & 6 & 19 \\
\hline 7 & e630 & 3 & 5.1 & 1,260 & 4 & 14 & 1,170 & 6 & 19 \\
\hline 11 & e900 & 4 & 9.7 & 1,190 & 4 & 13 & 1,290 & 6 & 21 \\
\hline 12 & e950 & 4 & 10 & 1,220 & 5 & 16 & 1,340 & 6 & 22 \\
\hline 13 & $\mathrm{e} 1,050$ & 3 & 8.5 & 1,210 & 5 & 16 & 1,390 & 6 & 23 \\
\hline 14 & $\mathrm{e} 1,000$ & 3 & 8.1 & 1,230 & 5 & 17 & 1,340 & 9 & 33 \\
\hline 15 & e830 & 3 & 6.7 & 1,200 & 6 & 19 & 1,330 & 8 & 29 \\
\hline 16 & e700 & 3 & 5.7 & 1,120 & 7 & 21 & 1,320 & 7 & 25 \\
\hline 17 & e 800 & 3 & 6.5 & 1,030 & 8 & 22 & 1,360 & 7 & 26 \\
\hline 18 & e1,000 & 5 & 14 & 1,030 & 7 & 19 & 1,350 & 9 & 33 \\
\hline 24 & 1,920 & 6 & 31 & 1,100 & 2 & 5.9 & 1,280 & 4 & 14 \\
\hline 25 & 1,740 & 4 & 19 & 1,100 & 2 & 5.9 & 1,240 & 6 & 20 \\
\hline 26 & 1,690 & 4 & 18 & 1,110 & 3 & 9.0 & 1,260 & 6 & 20 \\
\hline 27 & 1,660 & 4 & 18 & 1,110 & 3 & 9.0 & 1,260 & 6 & 20 \\
\hline 28 & 1,650 & 5 & 22 & 1,090 & 4 & 12 & 1,420 & 8 & 31 \\
\hline 29 & 1,630 & 5 & 22 & --- & --- & --- & 1,600 & 10 & 43 \\
\hline 30 & 1,610 & 5 & 22 & --- & --- & --- & 1,650 & 9 & 40 \\
\hline 31 & 1,530 & 8 & 33 & --- & --- & --- & 1,600 & 10 & 43 \\
\hline Total & 36,380 & --- & 524.3 & 33,840 & --- & 418.8 & 40,400 & --- & 750 \\
\hline Mean & 1,174 & 5 & 17 & 1,209 & 5 & 15 & 1,303 & 7 & 24 \\
\hline Max & 2,100 & 12 & 51 & 1,470 & 8 & 24 & 1,650 & 10 & 43 \\
\hline Min & 500 & 3 & 4.0 & 1,030 & 2 & 5.9 & 1,100 & 4 & 12 \\
\hline
\end{tabular}


Table 7. Daily mean streamflow and suspended-sediment data for Clark Fork above Missoula, Montana, October 2004 through September 2005.-Continued

[Abbreviations: acre-ft, acre-feet; $\mathrm{ft}^{3} / \mathrm{s}$, cubic feet per second; e, estimated; max, maximum; mg/L, milligrams per liter; min, minimum; ton/d, tons per day. Symbol: ---, no data or value not computed]

\begin{tabular}{|c|c|c|c|c|c|c|c|c|c|}
\hline \multirow{3}{*}{ Day } & \multirow{2}{*}{$\begin{array}{c}\text { Mean } \\
\text { stream- } \\
\text { flow } \\
\left(\mathrm{ft}^{3} / \mathrm{s}\right)\end{array}$} & \multicolumn{2}{|c|}{ Suspended sediment } & \multirow{2}{*}{$\begin{array}{c}\text { Mean } \\
\text { stream- } \\
\text { flow } \\
\left(\mathrm{ft}^{3} / \mathrm{s}\right)\end{array}$} & \multicolumn{2}{|c|}{ Suspended sediment } & \multirow{2}{*}{$\begin{array}{c}\text { Mean } \\
\text { stream- } \\
\text { flow } \\
\left(\mathrm{ft}^{3} / \mathrm{s}\right)\end{array}$} & \multicolumn{2}{|c|}{ Suspended sediment } \\
\hline & & $\begin{array}{c}\text { Mean } \\
\text { concentration } \\
\text { (mg/L) }\end{array}$ & $\begin{array}{l}\text { Discharge } \\
\text { (ton/d) }\end{array}$ & & $\begin{array}{c}\text { Mean } \\
\text { concentration } \\
(\mathrm{mg} / \mathrm{L})\end{array}$ & $\begin{array}{l}\text { Discharge } \\
\text { (ton/d) }\end{array}$ & & $\begin{array}{c}\text { Mean } \\
\text { concentration } \\
(\mathrm{mg} / \mathrm{L})\end{array}$ & $\begin{array}{c}\text { Discharge } \\
\text { (ton/d) }\end{array}$ \\
\hline & & April & & & May & & & June & \\
\hline 1 & 1,560 & 10 & 42 & 3,450 & 10 & 93 & 5,700 & 18 & 277 \\
\hline 4 & 1,660 & 8 & 36 & 2,970 & 8 & 64 & 8,800 & 81 & 1,920 \\
\hline 5 & 1,670 & 8 & 36 & 3,000 & 9 & 73 & 9,050 & 90 & 2,200 \\
\hline 6 & 1,660 & 8 & 36 & 3,240 & 8 & 70 & 8,980 & 68 & 1,650 \\
\hline 7 & 1,680 & 8 & 36 & 3,840 & 12 & 124 & 8,890 & 60 & 1,440 \\
\hline 11 & 2,080 & 11 & 62 & 6,630 & 48 & 859 & 6,380 & 25 & 431 \\
\hline 12 & 2,080 & 9 & 51 & 7,600 & 116 & 2,380 & 6,310 & 20 & 341 \\
\hline 13 & 2,070 & 8 & 45 & 7,410 & 114 & 2,280 & 6,780 & 28 & 513 \\
\hline 14 & 2,240 & 8 & 48 & 6,740 & 65 & 1,180 & 6,320 & 28 & 478 \\
\hline 15 & 2,280 & 9 & 55 & 6,660 & 55 & 989 & 5,970 & 22 & 355 \\
\hline 16 & 2,110 & 10 & 57 & 7,060 & 50 & 953 & 5,950 & 23 & 369 \\
\hline 17 & 2,200 & 8 & 48 & 8,330 & 66 & 1,480 & 6,130 & 23 & 381 \\
\hline 18 & 2,310 & 10 & 62 & 9,070 & 80 & 1,960 & 7,050 & 54 & 1,030 \\
\hline 24 & 2,610 & 10 & 70 & 7,800 & 46 & 969 & 5,170 & 15 & 209 \\
\hline 25 & 2,980 & 9 & 72 & 7,150 & 36 & 695 & 4,790 & 17 & 220 \\
\hline 26 & 3,450 & 13 & 121 & 6,600 & 31 & 552 & 4,670 & 16 & 202 \\
\hline 27 & 3,930 & 21 & 223 & 6,070 & 26 & 426 & 4,670 & 14 & 177 \\
\hline 28 & 4,000 & 14 & 151 & 5,790 & 22 & 344 & 5,020 & 16 & 217 \\
\hline 29 & 3,840 & 13 & 135 & 5,680 & 18 & 276 & 5,170 & 18 & 251 \\
\hline 30 & 3,630 & 12 & 118 & 5,640 & 18 & 274 & 4,970 & 16 & 215 \\
\hline 31 & --- & --- & --- & 5,540 & 18 & 269 & --- & --- & --- \\
\hline Total & 70,840 & --- & 2,022 & 191,360 & --- & 24,719 & 195,090 & --- & 19,365 \\
\hline Mean & 2,361 & 10 & 67 & 6,173 & 41 & 797 & 6,503 & 33 & 646 \\
\hline $\operatorname{Max}$ & 4,000 & 21 & 223 & 9,170 & 116 & 2,380 & 9,050 & 90 & 2,200 \\
\hline Min & 1,560 & 8 & 34 & 2,970 & 8 & 64 & 4,670 & 14 & 177 \\
\hline
\end{tabular}


Table 7. Daily mean streamflow and suspended-sediment data for Clark Fork above Missoula, Montana, 0ctober 2004 through September 2005.-Continued

[Abbreviations: acre-ft, acre-feet; $\mathrm{ft}^{3} / \mathrm{s}$, cubic feet per second; e, estimated; max, maximum; mg/L, milligrams per liter; min, minimum; ton/d, tons per day. Symbol: ---, no data or value not computed]

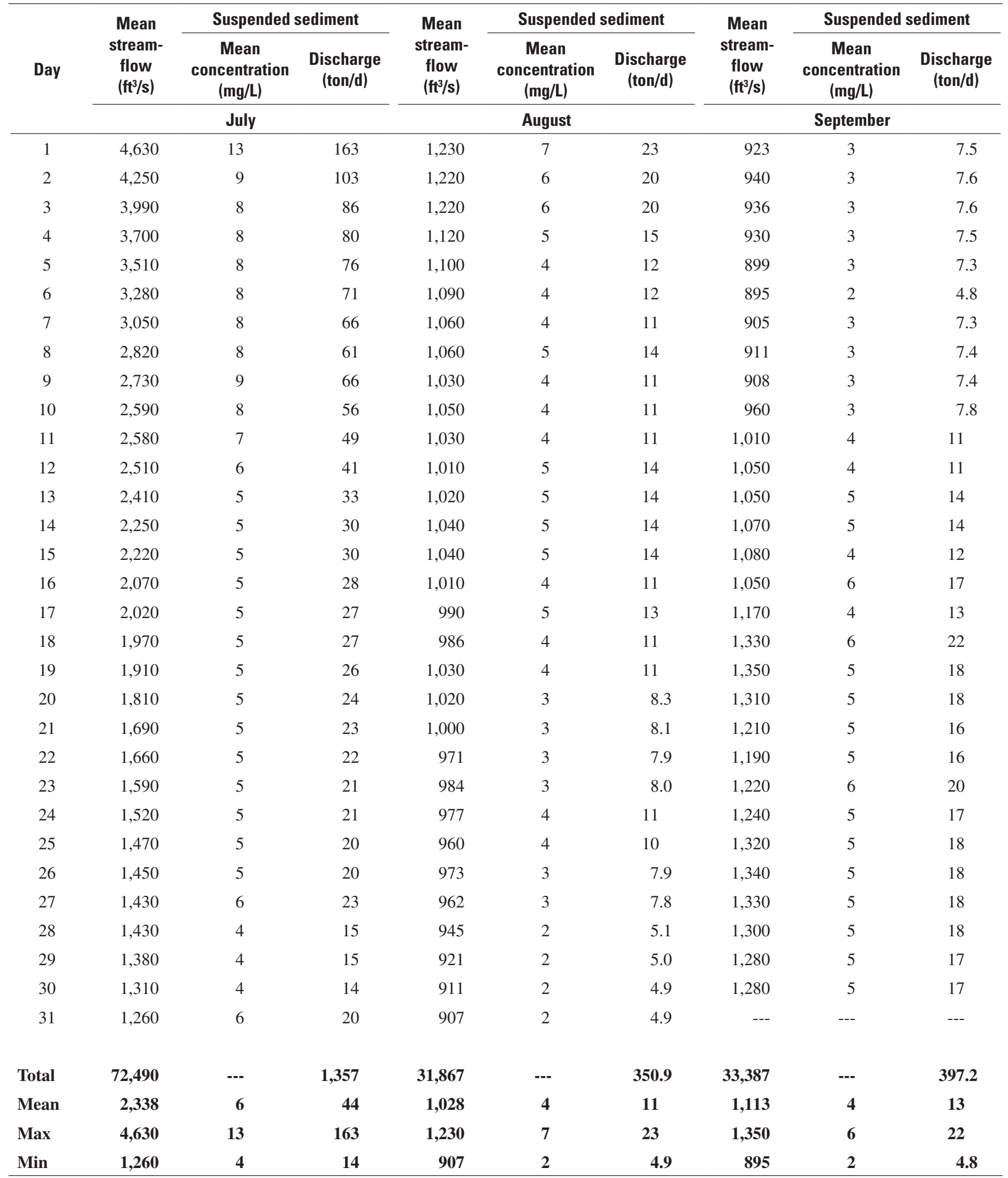

Total for water year 2005 (unrounded sum of daily values): streamflow, 826,764 ft³/s (1,640,000 acre-ft); sediment load, 51,051.1 tons. 
48 Water-Quality, Bed-Sediment, Biological Data, and Statistical Summaries, Upper Clark Fork Basin, Montana

Table 8. Analyses of field replicates for water samples, upper Clark Fork basin, Montana.

[Abbreviations: E, estimated; $\mu \mathrm{g} / \mathrm{L}$, micrograms per liter; mg/L, milligrams per liter; mm, millimeter. Symbol: <, less than laboratory reporting level]

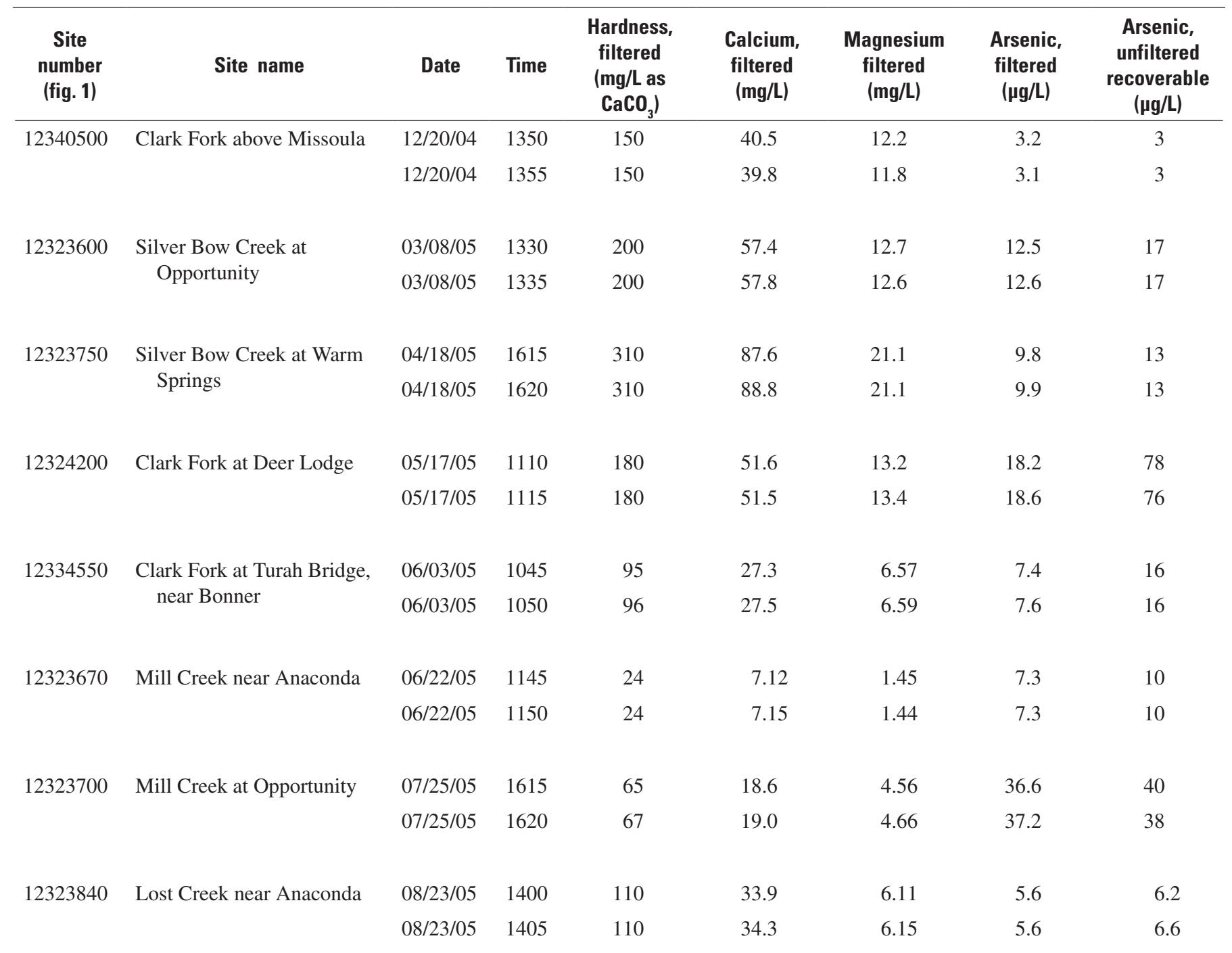


Table 8. Analyses of field replicates for water samples, upper Clark Fork basin, Montana.-Continued

[Abbreviations: E, estimated; $\mu \mathrm{g} / \mathrm{L}$, micrograms per liter; mg/L, milligrams per liter; mm, millimeter. Symbol: <, less than laboratory reporting level]

\begin{tabular}{|c|c|c|c|c|c|c|c|c|c|}
\hline $\begin{array}{c}\text { Site } \\
\text { number }\end{array}$ & Date & $\begin{array}{c}\text { Cadmium, } \\
\text { filtered } \\
(\mu \mathrm{g} / \mathrm{L})\end{array}$ & $\begin{array}{c}\text { Cadmium, } \\
\text { unfiltered } \\
\text { recoverable } \\
(\mu \mathrm{g} / \mathrm{L})\end{array}$ & $\begin{array}{l}\text { Copper, } \\
\text { filtered } \\
(\mu \mathrm{g} / \mathrm{L})\end{array}$ & $\begin{array}{c}\text { Copper, } \\
\text { unfiltered } \\
\text { recoverable } \\
(\mu \mathrm{g} / \mathrm{L})\end{array}$ & $\begin{array}{l}\text { Iron, } \\
\text { filtered } \\
(\mu \mathrm{g} / \mathrm{L})\end{array}$ & $\begin{array}{c}\text { Iron, } \\
\text { unfiltered } \\
\text { recoverable } \\
(\mu \mathrm{g} / \mathrm{L})\end{array}$ & $\begin{array}{l}\text { Lead, } \\
\text { filtered } \\
\text { ( } \mu \mathrm{g} / \mathrm{L})\end{array}$ & $\begin{array}{c}\text { Lead, } \\
\text { unfiltered } \\
\text { recoverable } \\
(\mu \mathrm{g} / \mathrm{L})\end{array}$ \\
\hline \multirow[t]{2}{*}{12340500} & $12 / 20 / 04$ & E0.03 & 0.04 & 1.4 & 3.3 & $<6$ & 70 & $<0.08$ & 0.43 \\
\hline & $12 / 20 / 04$ & E.03 & E.03 & 1.4 & 3.5 & $<6$ & 80 & $<.08$ & .43 \\
\hline \multirow[t]{2}{*}{12323600} & 03/08/05 & .78 & 1.29 & 32.7 & 93.2 & 9 & 550 & .30 & 15.1 \\
\hline & 03/08/05 & .85 & 1.25 & 32.5 & 94.9 & 9 & 560 & .30 & 15.0 \\
\hline \multirow[t]{2}{*}{12323750} & 04/18/05 & E.03 & .08 & 2.7 & 7.8 & E4 & 250 & E.05 & 1.09 \\
\hline & 04/18/05 & E.03 & .09 & 2.8 & 7.5 & E5 & 250 & E.05 & 1.13 \\
\hline \multirow[t]{2}{*}{12324200} & 05/17/05 & E.04 & 2.06 & 10.6 & 468 & 41 & 6,960 & .40 & 61.7 \\
\hline & 05/17/05 & E.04 & 2.05 & 11.2 & 478 & 44 & 6,910 & .43 & 62.0 \\
\hline \multirow[t]{2}{*}{12334550} & 06/03/05 & E.04 & .40 & 6.9 & 62.8 & 46 & 1,700 & .24 & 10.9 \\
\hline & 06/03/05 & E.04 & .41 & 7.2 & 63.8 & 44 & 1,680 & .25 & 11.1 \\
\hline \multirow[t]{2}{*}{12323670} & $06 / 22 / 05$ & .04 & .15 & 2.4 & 7.2 & 26 & 590 & .08 & 2.35 \\
\hline & $06 / 22 / 05$ & .04 & .17 & 2.4 & 7.8 & 29 & 580 & .09 & 2.38 \\
\hline \multirow[t]{2}{*}{12323700} & $07 / 25 / 05$ & .06 & .08 & 3.2 & 4.5 & 90 & 140 & .23 & .40 \\
\hline & 07/25/05 & .07 & .09 & 3.1 & 4.5 & 96 & 130 & .23 & .40 \\
\hline \multirow[t]{2}{*}{12323840} & $08 / 23 / 05$ & .05 & .08 & 3.6 & 8.9 & $<6$ & 160 & E.05 & .70 \\
\hline & $08 / 23 / 05$ & .05 & .07 & 3.8 & 9.3 & 7 & 150 & E.05 & .72 \\
\hline
\end{tabular}


50 Water-Quality, Bed-Sediment, Biological Data, and Statistical Summaries, Upper Clark Fork Basin, Montana

Table 8. Analyses of field replicates for water samples, upper Clark Fork basin, Montana.—Continued

[Abbreviations: E, estimated; $\mu \mathrm{g} / \mathrm{L}$, micrograms per liter; mg/L, milligrams per liter; mm, millimeter. Symbol: <, less than laboratory reporting level]

\begin{tabular}{|c|c|c|c|c|c|c|c|}
\hline $\begin{array}{c}\text { Site } \\
\text { number }\end{array}$ & Date & $\begin{array}{c}\text { Manganese, } \\
\text { filtered } \\
(\mu \mathrm{g} / \mathrm{L})\end{array}$ & $\begin{array}{c}\text { Manganese, } \\
\text { unfiltered } \\
\text { recoverable } \\
(\mu \mathrm{g} / \mathrm{L})\end{array}$ & $\begin{array}{c}\text { Zinc, } \\
\text { filtered } \\
\text { ( } \mu \mathrm{g} / \mathrm{L})\end{array}$ & $\begin{array}{c}\text { Zinc, } \\
\text { unfiltered } \\
\text { recoverable } \\
(\mu \mathrm{g} / \mathrm{L})\end{array}$ & $\begin{array}{c}\text { Sediment } \\
\text { suspended, } \\
\text { percent } \\
\text { finer than } \\
0.062 \mathrm{~mm}\end{array}$ & $\begin{array}{c}\text { Sediment, } \\
\text { suspended } \\
(\mathrm{mg} / \mathrm{L})\end{array}$ \\
\hline \multirow[t]{2}{*}{12340500} & $12 / 20 / 04$ & 7.5 & 12 & 2.3 & 6 & 86 & 5 \\
\hline & $12 / 20 / 04$ & 7.4 & 12 & 2.0 & 6 & 86 & 4 \\
\hline \multirow[t]{2}{*}{12323600} & 03/08/05 & 489 & 482 & 157 & 263 & 86 & 11 \\
\hline & 03/08/05 & 498 & 497 & 160 & 271 & 82 & 12 \\
\hline \multirow[t]{2}{*}{12323750} & 04/18/05 & 195 & 245 & 4.7 & 12 & 82 & 3 \\
\hline & 04/18/05 & 197 & 246 & 4.0 & 11 & 91 & 3 \\
\hline \multirow[t]{2}{*}{12324200} & 05/17/05 & 97.9 & 1,010 & 9.3 & 359 & 67 & 387 \\
\hline & 05/17/05 & 104 & 995 & 9.6 & 366 & 66 & 399 \\
\hline \multirow[t]{2}{*}{12334550} & 06/03/05 & 7.5 & 221 & 5.9 & 93 & 68 & 110 \\
\hline & 06/03/05 & 7.9 & 224 & 6.9 & 95 & 66 & 114 \\
\hline \multirow[t]{2}{*}{12323670} & $06 / 22 / 05$ & 5.1 & 36 & 1.6 & 7 & 57 & 29 \\
\hline & 06/22/05 & 5.1 & 38 & 1.7 & 7 & 55 & 30 \\
\hline \multirow[t]{2}{*}{12323700} & $07 / 25 / 05$ & 11.1 & 14 & 2.8 & 3 & 77 & 1 \\
\hline & $07 / 25 / 05$ & 11.4 & 14 & 2.1 & 3 & 82 & 1 \\
\hline \multirow[t]{2}{*}{12323840} & 08/23/05 & 1.2 & 6 & 1.3 & 4 & 80 & 8 \\
\hline & 08/23/05 & 1.3 & 6 & 1.4 & 3 & 86 & 11 \\
\hline
\end{tabular}


Table 9. Precision of analyses of field replicates for water samples, upper Clark Fork basin, Montana.

[Abbreviations: $\mu \mathrm{g} / \mathrm{L}$, micrograms per liter; $\mathrm{mg} / \mathrm{L}$, milligrams per liter; mm, millimeter]

\begin{tabular}{lccc}
\hline \multicolumn{1}{c}{ Constituent and reporting unit } & $\begin{array}{c}\text { Number of replicate } \\
\text { pairs }\end{array}$ & $\begin{array}{c}\text { Standard deviation', } \\
\text { in listed units }\end{array}$ & $\begin{array}{c}\text { Relative standard } \\
\text { deviation, } \\
\text { in percent }\end{array}$ \\
\hline Calcium, filtered, $\mathrm{mg} / \mathrm{L}$ & 8 & 0.39 & 0.97 \\
Magnesium, filtered, $\mathrm{mg} / \mathrm{L}$ & 8 & .12 & 1.2 \\
Arsenic, filtered, $\mu \mathrm{g} / \mathrm{L}$ & 8 & .19 & 1.5 \\
Arsenic, unfiltered recoverable, $\mu \mathrm{g} / \mathrm{L}$ & 8 & .71 & 3.1 \\
Cadmium, filtered, $\mu \mathrm{g} / \mathrm{L}$ & 8 & .02 & 13 \\
Cadmium, unfiltered recoverable, $\mu \mathrm{g} / \mathrm{L}$ & 8 & .01 & 2.4 \\
Copper, filtered, $\mu \mathrm{g} / \mathrm{L}$ & 8 & .19 & 2.3 \\
Copper, unfiltered recoverable, $\mu \mathrm{g} / \mathrm{L}$ & 8 & 2.6 & 3.1 \\
Iron, filtered, $\mu \mathrm{g} / \mathrm{L}$ & 8 & 2.2 & 7.5 \\
Iron, unfiltered recoverable, $\mu \mathrm{g} / \mathrm{L}$ & 8 & 15 & 1.1 \\
Lead, filtered, $\mu \mathrm{g} / \mathrm{L}$ & 8 & .01 & 4.7 \\
Lead, unfiltered recoverable, $\mu \mathrm{g} / \mathrm{L}$ & 8 & .09 & .81 \\
Manganese, filtered, $\mu \mathrm{g} / \mathrm{L}$ & 8 & 2.8 & 2.7 \\
Manganese, unfiltered recoverable, $\mu \mathrm{g} / \mathrm{L}$ & 8 & 5.4 & 2.1 \\
Zinc, filtered, $\mu \mathrm{g} / \mathrm{L}$ & 8 & .84 & 3.6 \\
Zinc, unfiltered recoverable, $\mu \mathrm{g} / \mathrm{L}$ & 8 & 2.7 & 2.9 \\
Sediment, suspended, percent finer than $0.062 \mathrm{~mm}$ & 8 & 3.2 & 4.2 \\
Sediment, suspended, $\mathrm{mg} / \mathrm{L}$ & 8 & 3.3 & 4.7 \\
\hline
\end{tabular}

${ }^{1}$ Standard deviation was calculated using the laboratory reporting level for censored values. 
Table 10. Precision of analyses of laboratory replicates for water samples, upper Clark Fork basin, Montana.

[Abbreviations: $\mu \mathrm{g} / \mathrm{L}$, micrograms per liter; mg/L, milligrams per liter]

\begin{tabular}{|c|c|c|c|c|}
\hline Constituent and reporting unit & $\begin{array}{c}\text { Number of replicate } \\
\text { pairs }\end{array}$ & $\begin{array}{l}\text { Standard deviation', } \\
\text { in listed units }\end{array}$ & $\begin{array}{c}\text { Relative standard } \\
\text { deviation, } \\
\text { in percent }\end{array}$ & $\begin{array}{c}\text { Within limits of } \\
\text { data-quality } \\
\text { objective }^{2}\end{array}$ \\
\hline Calcium, filtered, $\mathrm{mg} / \mathrm{L}$ & 8 & 0.88 & 2.3 & Yes \\
\hline Magnesium, filtered, mg/L & 8 & .10 & 1.1 & Yes \\
\hline Arsenic, filtered, $\mu \mathrm{g} / \mathrm{L}$ & 8 & .12 & 2.5 & Yes \\
\hline Arsenic, unfiltered recoverable, $\mu \mathrm{g} / \mathrm{L}$ & 7 & .52 & 7.2 & Yes \\
\hline Cadmium, filtered, $\mu \mathrm{g} / \mathrm{L}$ & 8 & .00 & 11 & Yes \\
\hline Cadmium, unfiltered recoverable, $\mu \mathrm{g} / \mathrm{L}$ & 8 & .00 & 4.6 & Yes \\
\hline Copper, filtered, $\mu \mathrm{g} / \mathrm{L}$ & 8 & .08 & 2.0 & Yes \\
\hline Copper, unfiltered recoverable, $\mu \mathrm{g} / \mathrm{L}$ & 8 & .14 & .67 & Yes \\
\hline Iron, filtered, $\mu \mathrm{g} / \mathrm{L}$ & 8 & 3.0 & 9.6 & Yes \\
\hline Iron, unfiltered recoverable, $\mu \mathrm{g} / \mathrm{L}$ & 8 & 13 & 2.3 & Yes \\
\hline Lead, filtered, $\mu \mathrm{g} / \mathrm{L}$ & 8 & .02 & 4.7 & Yes \\
\hline Lead, unfiltered recoverable, $\mu \mathrm{g} / \mathrm{L}$ & 8 & .05 & 1.9 & Yes \\
\hline Manganese, filtered, $\mu \mathrm{g} / \mathrm{L}$ & 8 & 2.8 & 6.6 & Yes \\
\hline Manganese, unfiltered recoverable, $\mu \mathrm{g} / \mathrm{L}$ & 8 & 7.5 & 3.8 & Yes \\
\hline Zinc, filtered, $\mu \mathrm{g} / \mathrm{L}$ & 8 & .30 & 7.4 & Yes \\
\hline Zinc, unfiltered recoverable, $\mu \mathrm{g} / \mathrm{L}$ & 8 & .22 & 1.7 & Yes \\
\hline
\end{tabular}

${ }^{1}$ Standard deviation was calculated using the laboratory reporting level for censored values.

${ }^{2}$ Data-quality objective for an acceptable level of precision is a maximum relative deviation of 20 percent for laboratory replicate analyses (table 3 ).

Table 11. Recovery efficiency for analyses of laboratory-spiked deionized-water blanks.

[Abbreviation: $\mu \mathrm{g} / \mathrm{L}$, micrograms per liter]

\begin{tabular}{lcccc}
\hline \multicolumn{1}{c}{ Constituent and reporting unit } & Number of samples & $\begin{array}{c}\text { 95-percent } \\
\text { confidence interval } \\
\text { for spike recovery, } \\
\text { in percent }\end{array}$ & $\begin{array}{c}\text { Mean spike } \\
\text { recovery, } \\
\text { in percent }\end{array}$ & $\begin{array}{c}\text { Within limits } \\
\text { of data-quality } \\
\text { objective' }\end{array}$ \\
\hline Arsenic, filtered, $\mu \mathrm{g} / \mathrm{L}$ & 5 & $95.5-99.3$ & 97.4 & Yes \\
Arsenic, unfiltered recoverable, $\mu \mathrm{g} / \mathrm{L}$ & 5 & $90.7-111$ & 101 & Yes \\
Cadmium, filtered, $\mu \mathrm{g} / \mathrm{L}$ & 5 & $97.3-106$ & 102 & Yes \\
Cadmium, unfiltered recoverable, $\mu \mathrm{g} / \mathrm{L}$ & 5 & $88.1-107$ & 97.5 & Yes \\
Copper, filtered, $\mu \mathrm{g} / \mathrm{L}$ & 5 & $97.4-107$ & 102 & Yes \\
Copper, unfiltered recoverable, $\mu \mathrm{g} / \mathrm{L}$ & 5 & $88.4-107$ & 97.6 & Yes \\
Iron, filtered, $\mu \mathrm{g} / \mathrm{L}$ & 5 & $95.2-105$ & 100 & Yes \\
Iron, unfiltered recoverable, $\mu \mathrm{g} / \mathrm{L}$ & 5 & $90.5-105$ & 97.6 & Yes \\
Lead, filtered, $\mu \mathrm{g} / \mathrm{L}$ & 5 & $95.5-106$ & 101 & Yes \\
Lead, unfiltered recoverable, $\mu \mathrm{g} / \mathrm{L}$ & 5 & $99.4-108$ & 104 & Yes \\
Manganese, filtered, $\mu \mathrm{g} / \mathrm{L}$ & 5 & $86.4-117$ & 102 & Yes \\
Manganese, unfiltered recoverable, $\mu \mathrm{g} / \mathrm{L}$ & 5 & $82.2-105$ & 93.8 & Yes \\
Zinc, filtered, $\mu \mathrm{g} / \mathrm{L}$ & 5 & $88.3-116$ & 102 & Yes \\
Zinc, unfiltered recoverable, $\mu \mathrm{g} / \mathrm{L}$ & 5 & $83.5-109$ & 96.1 & Yes \\
\hline
\end{tabular}

${ }^{1}$ Data-quality objective for acceptable bias is a maximum deviation of 25 percent from a theoretical 100-percent recovery (table 3 ). 
Table 12. Recovery efficiency for analyses of laboratory-spiked stream samples, upper Clark Fork basin, Montana.

[Abbreviation: $\mu \mathrm{g} / \mathrm{L}$, micrograms per liter]

\begin{tabular}{lcccc}
\hline \multicolumn{1}{c}{ Constituent and reporting unit } & Number of samples & $\begin{array}{c}\text { 95-percent } \\
\text { confidence interval } \\
\text { for spike recovery, } \\
\text { in percent }\end{array}$ & $\begin{array}{c}\text { Mean spike } \\
\text { recovery, } \\
\text { in percent }\end{array}$ & $\begin{array}{c}\text { Within limits of } \\
\text { data-quality } \\
\text { objective' }\end{array}$ \\
\hline Arsenic, filtered, $\mu \mathrm{g} / \mathrm{L}$ & 5 & $98.3-107$ & 103 & Yes \\
Arsenic, unfiltered recoverable, $\mu \mathrm{g} / \mathrm{L}$ & 5 & $101-108$ & 104 & Yes \\
Cadmium, filtered, $\mu \mathrm{g} / \mathrm{L}$ & 5 & $62.7-133$ & 97.8 & Yes \\
Cadmium, unfiltered recoverable, $\mu \mathrm{g} / \mathrm{L}$ & 5 & $88.5-108$ & 98.2 & Yes \\
Copper, filtered, $\mu \mathrm{g} / \mathrm{L}$ & 5 & $57.9-129$ & 93.6 & Yes \\
Copper, unfiltered recoverable, $\mu \mathrm{g} / \mathrm{L}$ & 5 & $84.8-101$ & 93.0 & Yes \\
Iron, filtered, $\mu \mathrm{g} / \mathrm{L}$ & 5 & $95.6-103$ & 99.3 & Yes \\
Iron, unfiltered recoverable, $\mu \mathrm{g} / \mathrm{L}$ & 5 & $95.9-108$ & 102 & Yes \\
Lead, filtered, $\mu \mathrm{g} / \mathrm{L}$ & 5 & $96.1-109$ & 102 & Yes \\
Lead, unfiltered recoverable, $\mu \mathrm{g} / \mathrm{L}$ & 5 & $99.7-106$ & 103 & Yes \\
Manganese, filtered, $\mu \mathrm{g} / \mathrm{L}$ & 5 & $87.3-108$ & 97.5 & Yes \\
Manganese, unfiltered recoverable, $\mu \mathrm{g} / \mathrm{L}$ & 5 & $78.3-98.3$ & 88.3 & Yes \\
Zinc, filtered, $\mu \mathrm{g} / \mathrm{L}$ & 5 & $60.8-128$ & 94.3 & Yes \\
Zinc, unfiltered recoverable, $\mu \mathrm{gg} / \mathrm{L}$ & 5 & $80.8-102$ & 91.6 & Yes \\
\hline
\end{tabular}

${ }^{1}$ Data-quality objective for acceptable bias is a maximum deviation of 25 percent from a theoretical 100-percent recovery (table 3 ). 
Table 13. Analyses of field blanks for water samples.

[Abbreviations; ${ }^{\circ} \mathrm{C}$, degrees Celsius; $\mu \mathrm{g} / \mathrm{L}$, micrograms per liter; $\mu \mathrm{S} / \mathrm{cm}$, microsiemens per centimeter at $25^{\circ} \mathrm{C} ; \mathrm{mg} / \mathrm{L}$, milligrams per liter. Symbol: $<$, less than laboratory reporting level]

\begin{tabular}{|c|c|c|c|c|c|c|c|c|c|c|}
\hline Date & Time & $\begin{array}{c}\text { pH, } \\
\text { onsite } \\
\text { (standard } \\
\text { units) }\end{array}$ & $\begin{array}{c}\text { Specific } \\
\text { conduc- } \\
\text { tance, } \\
\text { onsite } \\
(\mu \mathrm{S} / \mathrm{cm})\end{array}$ & \multicolumn{2}{|c|}{$\begin{array}{c}\text { Calcium, } \\
\text { filtered } \\
\text { (mg/L) }\end{array}$} & $\begin{array}{l}\text { Magne- } \\
\text { sium, } \\
\text { filtered } \\
\text { (mg/L) }\end{array}$ & $\begin{array}{c}\text { Arsenic, } \\
\text { filtered } \\
(\mu \mathrm{g} / \mathrm{L})\end{array}$ & $\begin{array}{c}\text { Arsenic, } \\
\text { unfiltered } \\
\text { recoverable } \\
\text { ( } \mathrm{gg} / \mathrm{L})\end{array}$ & $\begin{array}{c}\text { Cadmium, } \\
\text { filtered } \\
(\mu \mathrm{g} / \mathrm{L})\end{array}$ & $\begin{array}{c}\text { Cadmium, } \\
\text { unfiltered } \\
\text { recoverable } \\
(\mu \mathrm{g} / \mathrm{L})\end{array}$ \\
\hline $12 / 15 / 2004$ & 1130 & 5.5 & 2 & \multicolumn{2}{|c|}{$<0.02$} & $<0.008$ & $<0.2$ & $<2$ & $<0.04$ & $<0.04$ \\
\hline 03/07/2005 & 1200 & 5.5 & 2 & \multicolumn{2}{|c|}{$<.02$} & $<.008$ & $<.2$ & $<2$ & .06 & $<.04$ \\
\hline 05/16/2005 & 1500 & 5.6 & 2 & \multicolumn{2}{|c|}{.02} & $<.008$ & $<.2$ & $<2$ & $<.04$ & $<.04$ \\
\hline 06/02/2005 & 1500 & 5.5 & 1 & \multicolumn{2}{|c|}{$<.02$} & $<.008$ & $<.2$ & $<2$ & $<.04$ & $<.04$ \\
\hline $06 / 23 / 2005$ & 0600 & 5.7 & 2 & \multicolumn{2}{|c|}{$<.02$} & $<.008$ & $<.2$ & $<2$ & $<.04$ & $<.04$ \\
\hline 07/26/2005 & 0600 & 5.7 & 2 & \multicolumn{2}{|c|}{$<.02$} & $<.008$ & $<.2$ & $<.12$ & $<.04$ & $<.04$ \\
\hline $12 / 15 / 2004$ & $<0.4$ & $<0.6$ & $<6$ & $<6$ & $<0.08$ & $<0.06$ & $<0.2$ & $<0.2$ & $<0.6$ & $<2$ \\
\hline 03/07/2005 & .5 & .7 & $<6$ & $<6$ & $<.08$ & $<.06$ & $<.2$ & $<.2$ & 1.9 & $<2$ \\
\hline $04 / 20 / 2005$ & $<.4$ & $<.6$ & $<6$ & $<6$ & $<.08$ & $<.06$ & $<.2$ & $<.2$ & .7 & $<2$ \\
\hline 05/16/2005 & $<.4$ & $<.6$ & $<6$ & $<6$ & $<.08$ & $<.06$ & $<.2$ & $<.2$ & $<.6$ & $<2$ \\
\hline 06/02/2005 & $<.4$ & $<.6$ & $<6$ & 30 & $<.08$ & $<.06$ & $<.2$ & $<.2$ & $<.6$ & $<2$ \\
\hline $06 / 23 / 2005$ & $<.4$ & $<.6$ & $<6$ & $<6$ & $<.08$ & $<.06$ & $<.2$ & $<.2$ & .9 & $<2$ \\
\hline
\end{tabular}




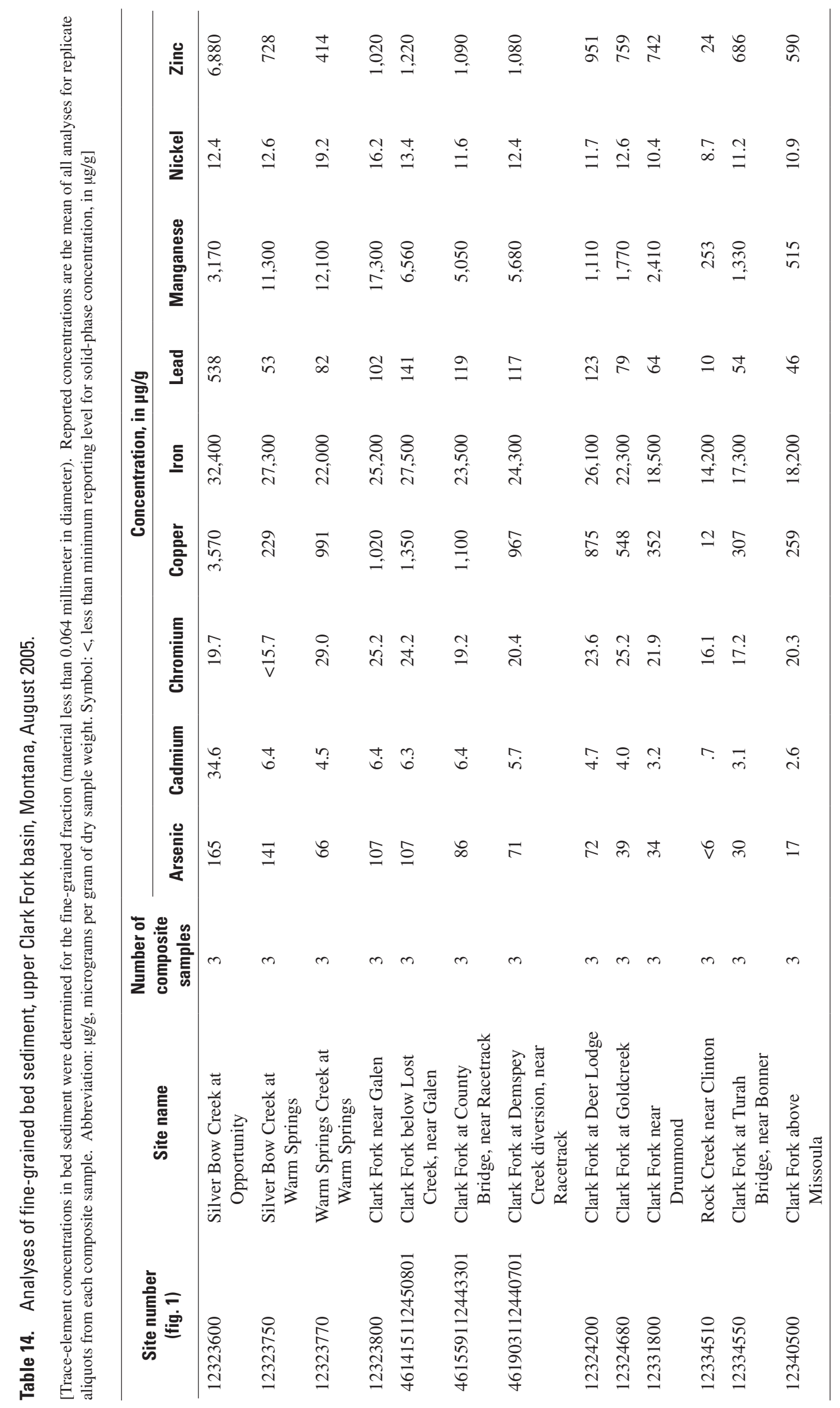


Table 15. Recovery efficiency for analyses of standard reference materials for bed sediment.

[Dilution ratio is the proportion of initial volume of concentrated nitric acid used as a digesting reagent to final volume of solution after addition of $0.6 \mathrm{~N}$ (normal) hydrochloric acid used for reconstituting dried residue. Abbreviations: $\mu \mathrm{g} / \mathrm{g}$, micrograms per gram of dry sample weight; SRM,standard reference material (agricultural soils). Symbol: --, recovery could not be determined because all analyses were less than the minimum reporting level]

\begin{tabular}{|c|c|c|c|c|c|}
\hline Constituent & $\begin{array}{c}\text { Number of } \\
\text { analyses }\end{array}$ & $\begin{array}{l}\text { Dilution } \\
\text { ratio }\end{array}$ & $\begin{array}{c}\text { Certified } \\
\text { concentration } \\
(\mu \mathrm{g} / \mathrm{g})\end{array}$ & $\begin{array}{c}\text { Mean SRM recovery, } \\
\text { in percent }\end{array}$ & $\begin{array}{c}\text { 95-percent } \\
\text { confidence interval } \\
\text { for SRM recovery, } \\
\text { in percent }\end{array}$ \\
\hline \multicolumn{6}{|c|}{ SRM sample 2709} \\
\hline Arsenic & 10 & $1: 10$ & 17.7 & 65.4 & $61.7-69.1$ \\
\hline Cadmium & 10 & $1: 10$ & .4 & -- & -- \\
\hline Chromium & 10 & $1: 10$ & 130 & 60.2 & $58.1-62.4$ \\
\hline Copper & 10 & $1: 10$ & 35 & 83.6 & $77.5-89.7$ \\
\hline Iron & 10 & $1: 10$ & 35,000 & 85.0 & $83.5-86.4$ \\
\hline Lead & 10 & $1: 10$ & 19 & 86.1 & $82.9-89.3$ \\
\hline Manganese & 10 & $1: 10$ & 538 & 89.1 & $87.7-90.6$ \\
\hline Nickel & 10 & $1: 10$ & 88 & 82.2 & $80.3-84.0$ \\
\hline Zinc & 10 & $1: 10$ & 106 & 75.6 & $72.9-78.3$ \\
\hline \multicolumn{6}{|c|}{ SRM sample 2711} \\
\hline Arsenic & 10 & $1: 10$ & 105 & 83.4 & $81.2-85.7$ \\
\hline Cadmium & 10 & $1: 10$ & 41.7 & 94.4 & $92.2-96.6$ \\
\hline Chromium & 10 & $1: 10$ & 47 & 51.5 & $49.1-53.9$ \\
\hline Copper & 10 & $1: 10$ & 114 & 93.7 & $92.1-95.3$ \\
\hline Iron & 10 & $1: 10$ & 28,900 & 80.7 & $79.3-82.1$ \\
\hline Lead & 10 & $1: 10$ & 1,160 & 88.9 & 86.9-90.9 \\
\hline Manganese & 10 & $1: 10$ & 638 & 80.6 & $79.4-81.7$ \\
\hline Nickel & 10 & $1: 10$ & 20.6 & 78.9 & $76.6-81.2$ \\
\hline Zinc & 10 & $1: 10$ & 350 & 88.1 & $86.7-89.4$ \\
\hline
\end{tabular}




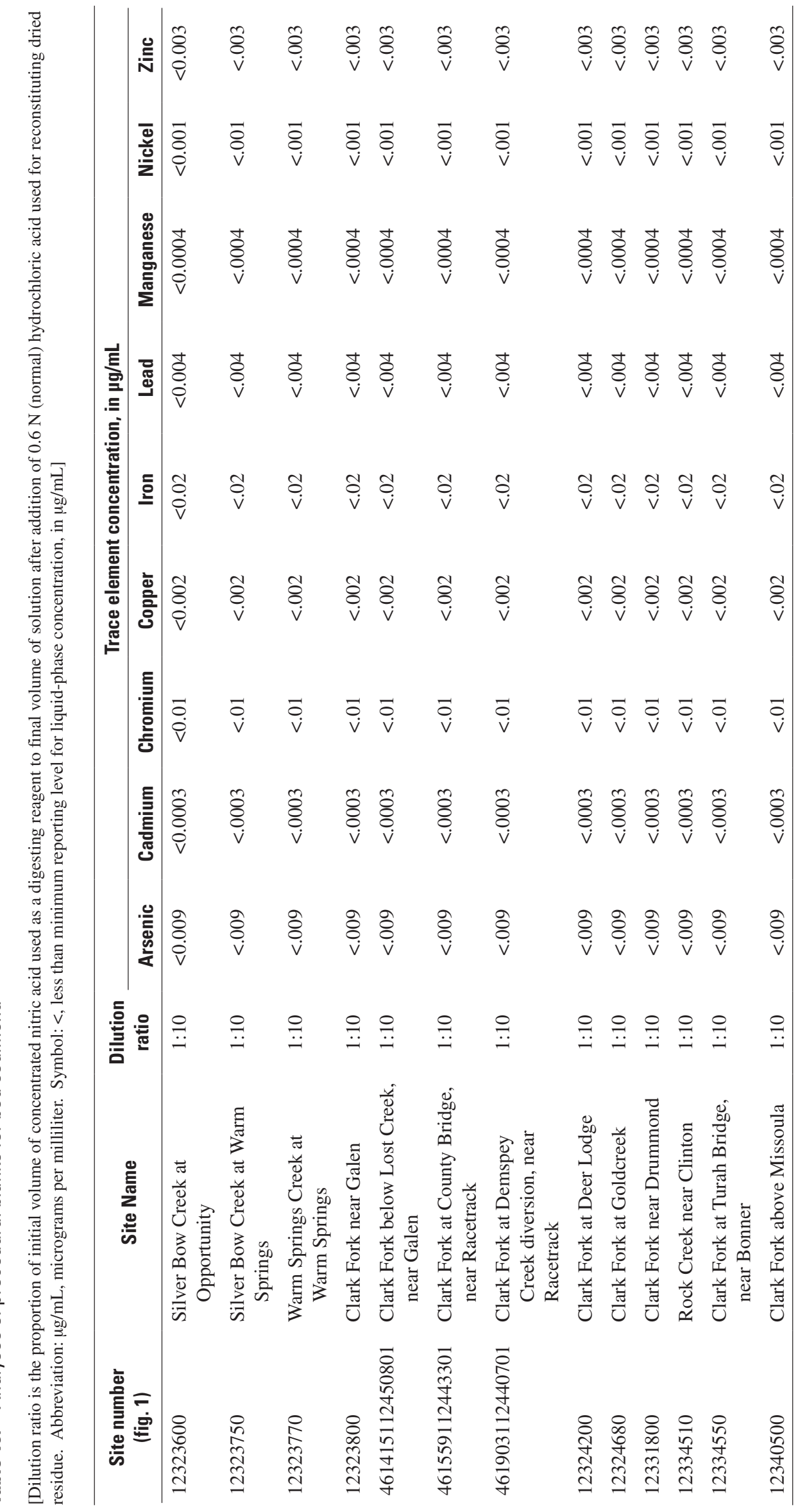


Table 17. Analyses of biota, upper Clark Fork basin, Montana, August 2005.

[Analyses are for the whole-body tissue of aquatic insects. Composite samples are made by combining similar-sized insects of the same species into a sample of sufficient mass for analysis. Concentrations for biota samples composed of two or more composite samples are the means of all analyses. Abbreviations: $\mu \mathrm{g} / \mathrm{g}$, micrograms per gram of dry sample weight; spp., species. Symbol: <, less than minimum reporting level for solid-phase concentration, in $\mu \mathrm{g} / \mathrm{g}$ ]

\begin{tabular}{|c|c|c|c|c|c|c|c|c|c|c|}
\hline \multirow[b]{2}{*}{ Taxon } & \multirow{2}{*}{$\begin{array}{l}\text { Number } \\
\text { of com- } \\
\text { posite } \\
\text { samples }\end{array}$} & \multicolumn{9}{|c|}{ Concentration, in $\mu \mathrm{g} / \mathrm{g}$} \\
\hline & & Arsenic & $\begin{array}{l}\text { Cad- } \\
\text { mium }\end{array}$ & $\begin{array}{l}\text { Chro- } \\
\text { mium }\end{array}$ & Copper & Iron & Lead & $\begin{array}{c}\text { Manga- } \\
\text { nese }\end{array}$ & Nickel & Zinc \\
\hline \multicolumn{11}{|c|}{12323600 -Silver Bow Creek at Opportunity } \\
\hline Hydropsyche cockerelli & 1 & 13.1 & 4.9 & 1.4 & 382 & 2,110 & 45.0 & 847 & 2.0 & 789 \\
\hline Hydropsyche spp. & 1 & 15.1 & 6.1 & $<9.7$ & 418 & 2,550 & 51.4 & 1,040 & 2.5 & 909 \\
\hline Hydropsyche cockerelli & 3 & 10.4 & .4 & $<2.6$ & 19.7 & 833 & 1.7 & 1,440 & 0.6 & 147 \\
\hline Hydropsyche occidentalis & 2 & 10.8 & .4 & $<5.4$ & 24.4 & 959 & 2.1 & 1,770 & .9 & 144 \\
\hline \multicolumn{11}{|c|}{12323770 - Warm Springs Creek at Warm Springs } \\
\hline Arctopsyche grandis & 2 & 9.6 & 3.4 & $<0.3$ & 130 & 1,080 & 5.2 & 3,180 & 2.5 & 197 \\
\hline Hydropsyche occidentalis & 1 & 12.5 & 1.3 & 1.7 & 92.4 & 1,450 & 8.4 & 3,940 & 1.5 & 198 \\
\hline \multicolumn{11}{|c|}{ 461415112450801—Clark Fork below Lost Creek, near Galen } \\
\hline Hydropsyche cockerelli & 2 & 9.5 & 1.8 & 1.1 & 124 & 1,150 & 7.1 & 1,700 & 1.1 & 195 \\
\hline \multicolumn{11}{|c|}{ 461559112443301—Clark Fork at County Bridge, near Racetrack } \\
\hline Hydropsyche cockerelli & 3 & 11.5 & 1.5 & 1.0 & 118 & 993 & 7.3 & 1,930 & 1.0 & 192 \\
\hline \multicolumn{11}{|c|}{ 461903112440701—Clark Fork at Dempsey Creek Diversion, near Racetrack } \\
\hline Hydropsyche cockerelli & 1 & 8.0 & 1.7 & 1.3 & 106 & 1,180 & 6.3 & 1,480 & 1.0 & 188 \\
\hline Hydropsyche occidentalis & 1 & 10.2 & 1.2 & 1.5 & 90.7 & 1,270 & 7.0 & 2,320 & 1.4 & 211 \\
\hline Claassenia sabulosa & 2 & 1.6 & .7 & $<1.2$ & 55.7 & 179 & .7 & 130 & .3 & 264 \\
\hline Hydropsyche cockerelli & 2 & 4.7 & 1.5 & 2.5 & 60.4 & 759 & 3.5 & 1,100 & 0.8 & 181 \\
\hline Hydropsyche occidentalis & 1 & 5.3 & 1.5 & $<2.6$ & 57.6 & 1,250 & 4.6 & 1,320 & 1.0 & 203 \\
\hline
\end{tabular}


Table 17. Analyses of biota, upper Clark Fork basin, Montana, August 2005.-Continued

[Analyses are for the whole-body tissue of aquatic insects. Composite samples are made by combining similar-sized insects of the same species into a sample of sufficient mass for analysis. Concentrations for biota samples composed of two or more composite samples are the means of all analyses. Abbreviations: $\mu \mathrm{g} / \mathrm{g}$, micrograms per gram of dry sample weight; spp., species. Symbol: <, less than minimum reporting level for solid-phase concentration, in $\mu \mathrm{g} / \mathrm{g}$ ]

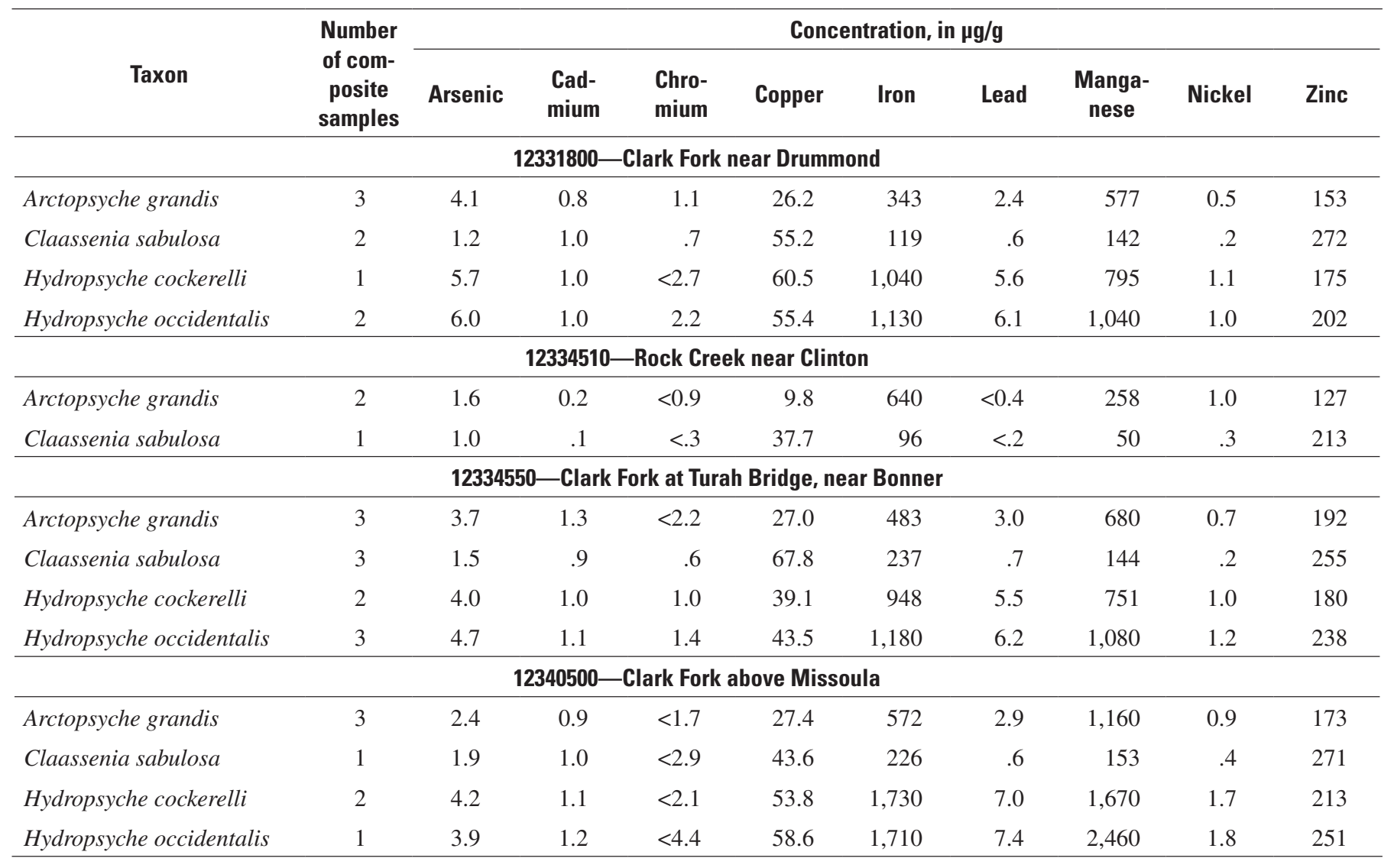


Table 18. Recovery efficiency for analyses of standard reference material for biota.

[Abbreviations: $\mu \mathrm{g} / \mathrm{g}$, micrograms per gram of dry sample weight; $\mu \mathrm{g} / \mathrm{mL}$, micrograms per milliliter; SRM, standard reference material (lobster hepatopancreas)]

\begin{tabular}{lcccc}
\hline Constituent & $\begin{array}{c}\text { Number of } \\
\text { analyses }\end{array}$ & $\begin{array}{c}\text { Certified } \\
\text { concentration } \\
(\boldsymbol{\mu g} / \mathbf{g})\end{array}$ & $\begin{array}{c}\text { Mean SRM } \\
\text { recovery, } \\
\text { in percent }\end{array}$ & $\begin{array}{c}\text { 95-percent confidence } \\
\text { interval for SRM recovery, } \\
\text { in percent }\end{array}$ \\
\hline SRM sample TORT-2 & \\
\hline Arsenic & 12 & 21.6 & 97.7 & $96.9-98.6$ \\
Cadmium & 12 & 26.7 & 92.6 & $91.4-93.8$ \\
Copper & 12 & .77 & 119 & $101-136$ \\
Iron & 12 & 106 & 96.7 & $95.9-97.6$ \\
Lead & 12 & 105 & 100 & $97.4-103$ \\
Manganese & 12 & .35 & 84.7 & $74.6-94.8$ \\
Nickel & 12 & 13.6 & 95.4 & $94.0-96.8$ \\
Zinc & 12 & 2.5 & 84.8 & $82.7-86.9$ \\
\hline
\end{tabular}

${ }^{1}$ Chromium concentrations in two analyses were less than the liquid-phase minimum reporting level $(0.03 \mu \mathrm{g} / \mathrm{mL})$ and omitted from the calculation of SRM recovery. 


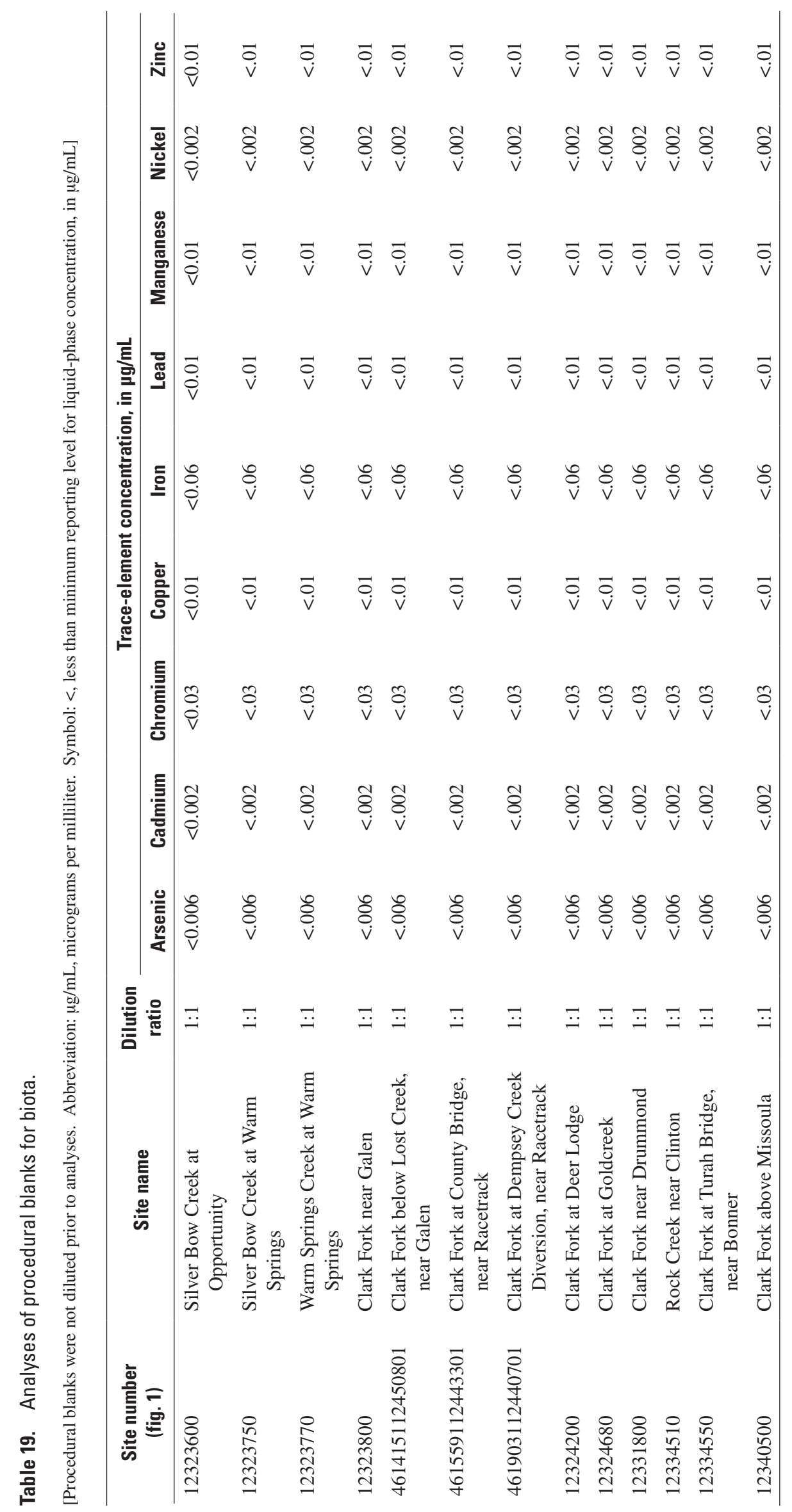


Table 20. Statistical summary of water-quality data for the upper Clark Fork basin, Montana, March 1985 through September 2005.

[Abbreviations: $\mathrm{ft}^{3} / \mathrm{s}$, cubic feet per second; ${ }^{\circ} \mathrm{C}$, degrees Celsius; E, estimated; $\mu \mathrm{g} / \mathrm{L}$, micrograms per liter; $\mu \mathrm{S} / \mathrm{cm}$, microsiemens per centimeter at $25^{\circ} \mathrm{C} ; \mathrm{mg} / \mathrm{L}$, milligrams per liter; $\mathrm{mm}$, millimeter; ton/d, tons per day. Symbols: $<$, less than laboratory reporting level ${ }^{1}$; --, indicates insufficient data greater than the laboratory reporting level to compute statistic]

\begin{tabular}{|c|c|c|c|c|c|}
\hline Property or constituent and reporting unit & $\begin{array}{c}\text { Number of } \\
\text { samples }\end{array}$ & Maximum & Minimum & Mean & Median \\
\hline \multicolumn{6}{|c|}{$\begin{array}{l}12323230 \text { —Blacktail Creek at Harrison Avenue, at Butte } \\
\text { Period of record for water-quality data: March 1993-August 1995, December 1996-August 2003, December 2004-September } 2005\end{array}$} \\
\hline Streamflow, instantaneous $\left(\mathrm{ft}^{3} / \mathrm{s}\right)$ & 91 & 156 & 1.9 & 14 & 7.6 \\
\hline $\mathrm{pH}$, onsite (standard units) & 91 & 8.4 & 7.3 & 7.8 & 7.8 \\
\hline Specific conductance, onsite $(\mu \mathrm{S} / \mathrm{cm})$ & 91 & 412 & 116 & 267 & 271 \\
\hline Temperature, water $\left({ }^{\circ} \mathrm{C}\right)$ & 91 & 17.5 & 1.5 & 8.2 & 8.0 \\
\hline Hardness, filtered $\left(\mathrm{mg} / \mathrm{L}\right.$ as $\left.\mathrm{CaCO}_{3}\right)$ & 91 & 150 & 38 & 105 & 110 \\
\hline Calcium, filtered (mg/L) & 91 & 41.8 & 10.6 & 30.0 & 30.9 \\
\hline Magnesium, filtered (mg/L) & 91 & 11.0 & 2.71 & 7.3 & 7.4 \\
\hline Arsenic, filtered $(\mu \mathrm{g} / \mathrm{L})$ & 91 & 13 & 1 & 3.8 & 3.0 \\
\hline Arsenic, unfiltered $(\mu \mathrm{g} / \mathrm{L})$ & 91 & 18 & 1 & ${ }^{25}$ & 4 \\
\hline Cadmium, filtered $(\mu \mathrm{g} / \mathrm{L})$ & 91 & .5 & $<.1$ & ${ }^{2} .06$ & $<.1$ \\
\hline Cadmium, unfiltered $(\mu \mathrm{g} / \mathrm{L})$ & 91 & .11 & $<.04$ & ${ }^{2} .05$ & $<1$ \\
\hline Copper, filtered $(\mu \mathrm{g} / \mathrm{L})$ & 91 & 10 & .8 & 23.8 & 3.2 \\
\hline Copper, unfiltered $(\mu \mathrm{g} / \mathrm{L})$ & 91 & 52 & 1.5 & 7.2 & 5.7 \\
\hline Iron, filtered $(\mu \mathrm{g} / \mathrm{L})$ & 91 & 478 & 15 & 157 & 150 \\
\hline Iron, unfiltered $(\mu \mathrm{g} / \mathrm{L})$ & 91 & 4,220 & 140 & 685 & 550 \\
\hline Lead, filtered $(\mu \mathrm{g} / \mathrm{L})$ & 91 & 2.8 & $<.08$ & ${ }^{2} .22$ & $<.6$ \\
\hline Lead, unfiltered $(\mu \mathrm{g} / \mathrm{L})$ & 91 & 47 & $<1$ & ${ }^{2} 2.31$ & .92 \\
\hline Manganese, filtered $(\mu \mathrm{g} / \mathrm{L})$ & 91 & 144 & 14.2 & 42.0 & 37.9 \\
\hline Manganese, unfiltered $(\mu \mathrm{g} / \mathrm{L})$ & 91 & 240 & 23 & 60 & 51 \\
\hline Zinc, filtered $(\mu \mathrm{g} / \mathrm{L})$ & 91 & 11 & $<1$ & 23.9 & 2.8 \\
\hline Zinc, unfiltered $(\mu \mathrm{g} / \mathrm{L})$ & 91 & 130 & $<10$ & ${ }^{2} 10$ & 4 \\
\hline Sediment, suspended (percent finer than $0.062 \mathrm{~mm}$ ) & 91 & 97 & 50 & 82 & 83 \\
\hline Sediment, suspended concentration (mg/L) & 91 & 139 & 2 & 14 & 8 \\
\hline Sediment, suspended discharge (ton/d) & 91 & 59 & .02 & 1.3 & .16 \\
\hline
\end{tabular}


Table 20. Statistical summary of water-quality data for the upper Clark Fork basin, Montana, March 1985 through September 2005. -Continued

[Abbreviations: $\mathrm{ft}^{3} / \mathrm{s}$, cubic feet per second; ${ }^{\circ} \mathrm{C}$, degrees Celsius; E, estimated; $\mu \mathrm{g} / \mathrm{L}$, micrograms per liter; $\mu \mathrm{S} / \mathrm{cm}, \mathrm{microsiemens}$ per centimeter at $25^{\circ} \mathrm{C}$; $\mathrm{mg} / \mathrm{L}$, milligrams per liter; mm, millimeter; ton/d, tons per day. Symbols: <, less than laboratory reporting level ${ }^{1}$; --, indicates insufficient data greater than the laboratory reporting level to compute statistic]

\begin{tabular}{|c|c|c|c|c|c|}
\hline Property or constituent and reporting unit & $\begin{array}{l}\text { Number of } \\
\text { samples }\end{array}$ & Maximum & Minimum & Mean & Median \\
\hline \multicolumn{6}{|c|}{$\begin{array}{l}12323250 \text {-Silver Bow Creek below Blacktail Creek, at Butte } \\
\text { Period of record for water-quality data: March 1993-August 1995, December 1996-September } 2005\end{array}$} \\
\hline Streamflow, instantaneous $\left(\mathrm{ft}^{3} / \mathrm{s}\right)$ & 99 & 134 & 13 & 28 & 23 \\
\hline $\mathrm{pH}$, onsite (standard units) & 99 & 8.1 & 7.2 & 7.6 & 7.6 \\
\hline Specific conductance, onsite $(\mu \mathrm{S} / \mathrm{cm})$ & 99 & 691 & 226 & 477 & 485 \\
\hline Temperature, water $\left({ }^{\circ} \mathrm{C}\right)$ & 99 & 20.0 & 1.0 & 10.4 & 9.5 \\
\hline Hardness, filtered (mg/L as $\left.\mathrm{CaCO}_{3}\right)$ & 99 & 220 & 66 & 151 & 160 \\
\hline Calcium, filtered (mg/L) & 99 & 62.7 & 19.0 & 43.1 & 44.0 \\
\hline Magnesium, filtered (mg/L) & 99 & 14.6 & 4.51 & 10.6 & 11.0 \\
\hline Arsenic, filtered $(\mu \mathrm{g} / \mathrm{L})$ & 99 & 13 & 2.3 & 6.7 & 6.5 \\
\hline Arsenic, unfiltered $(\mu \mathrm{g} / \mathrm{L})$ & 99 & 45 & 3 & 12 & 10 \\
\hline Cadmium, filtered $(\mu \mathrm{g} / \mathrm{L})$ & 99 & 6.2 & .09 & 1.37 & 1.10 \\
\hline Cadmium, unfiltered ( $\mu \mathrm{g} / \mathrm{L})$ & 99 & 6 & .11 & 1.83 & 1.60 \\
\hline Copper, filtered $(\mu \mathrm{g} / \mathrm{L})$ & 99 & 303 & 3.2 & 43.6 & 19.7 \\
\hline Copper, unfiltered $(\mu \mathrm{g} / \mathrm{L})$ & 99 & 550 & 13.5 & 102 & 62 \\
\hline Iron, filtered $(\mu \mathrm{g} / \mathrm{L})$ & 99 & 270 & 10 & 282 & 61 \\
\hline Iron, unfiltered $(\mu \mathrm{g} / \mathrm{L})$ & 99 & 7,400 & 90 & 1,000 & 640 \\
\hline Lead, filtered ( $\mu \mathrm{g} / \mathrm{L})$ & 99 & 2.4 & $<.5$ & ${ }^{2} .54$ & .20 \\
\hline Lead, unfiltered ( $\mu \mathrm{g} / \mathrm{L})$ & 99 & 250 & .65 & 16.0 & 5.80 \\
\hline Manganese, filtered ( $\mu \mathrm{g} / \mathrm{L})$ & 99 & 1,700 & 21.4 & 433 & 386 \\
\hline Manganese, unfiltered $(\mu \mathrm{g} / \mathrm{L})$ & 99 & 1,600 & 26 & 482 & 432 \\
\hline Zinc, filtered $(\mu \mathrm{g} / \mathrm{L})$ & 99 & 2,200 & 26.7 & 445 & 320 \\
\hline Zinc, unfiltered $(\mu \mathrm{g} / \mathrm{L})$ & 99 & 2,200 & 38 & 538 & 380 \\
\hline Sediment, suspended (percent finer than $0.062 \mathrm{~mm}$ ) & 98 & 98 & 42 & 84 & 86 \\
\hline Sediment, suspended concentration (mg/L) & 98 & 405 & 2 & 27 & 11 \\
\hline Sediment, suspended discharge (ton/d) & 98 & 70 & .09 & 3.2 & .82 \\
\hline
\end{tabular}


Table 20. Statistical summary of water-quality data for the upper Clark Fork basin, Montana, March 1985 through September 2005. -Continued

[Abbreviations: $\mathrm{ft}^{3} / \mathrm{s}$, cubic feet per second; ${ }^{\circ} \mathrm{C}$, degrees Celsius; E, estimated; $\mu \mathrm{g} / \mathrm{L}$, micrograms per liter; $\mu \mathrm{S} / \mathrm{cm}, \mathrm{microsiemens} \mathrm{per} \mathrm{centimeter} \mathrm{at} 25^{\circ} \mathrm{C}$; $\mathrm{mg} / \mathrm{L}$, milligrams per liter; mm, millimeter; ton/d, tons per day. Symbols: <, less than laboratory reporting level ${ }^{1}$; --, indicates insufficient data greater than the laboratory reporting level to compute statistic]

\begin{tabular}{|c|c|c|c|c|c|}
\hline Property or constituent and reporting unit & $\begin{array}{l}\text { Number of } \\
\text { samples }\end{array}$ & Maximum & Minimum & Mean & Median \\
\hline \multicolumn{6}{|c|}{$\begin{array}{l}12323600 \text {-Silver Bow Creek at Opportunity } \\
\text { Period of record for water-quality data: March 1993-August 1995, December 1996-September } 2005\end{array}$} \\
\hline Streamflow, instantaneous $\left(\mathrm{ft}^{3} / \mathrm{s}\right)$ & 102 & 361 & 13 & 72 & 50 \\
\hline pH, onsite (standard units) & 101 & 9.5 & 7.2 & 8.4 & 8.4 \\
\hline Hardness, filtered $\left(\mathrm{mg} / \mathrm{L}\right.$ as $\left.\mathrm{CaCO}_{3}\right)$ & 101 & 240 & 60 & 149 & 150 \\
\hline Calcium, filtered (mg/L) & 101 & 71.6 & 18.5 & 44.1 & 43.1 \\
\hline Magnesium, filtered (mg/L) & 101 & 15.0 & 3.42 & 9.44 & 9.04 \\
\hline Arsenic, filtered $(\mu \mathrm{g} / \mathrm{L})$ & 101 & 34 & 1 & 11.0 & 10.0 \\
\hline Copper, filtered $(\mu \mathrm{g} / \mathrm{L})$ & 101 & 450 & 18.5 & 51.9 & 40.2 \\
\hline Copper, unfiltered $(\mu \mathrm{g} / \mathrm{L})$ & 101 & 3,900 & 54.3 & 242 & 132 \\
\hline Iron, filtered $(\mu \mathrm{g} / \mathrm{L})$ & 101 & 307 & 3 & 244 & 23 \\
\hline Iron, unfiltered $(\mu \mathrm{g} / \mathrm{L})$ & 101 & 24,100 & 260 & 1,700 & 780 \\
\hline Lead, filtered $(\mu \mathrm{g} / \mathrm{L})$ & 101 & 5.1 & $<.5$ & ${ }^{2} .76$ & .27 \\
\hline Lead, unfiltered $(\mu \mathrm{g} / \mathrm{L})$ & 101 & 650 & 5.38 & 43.9 & 15.5 \\
\hline Manganese, filtered $(\mu \mathrm{g} / \mathrm{L})$ & 101 & 9,300 & 68 & 517 & 418 \\
\hline Manganese, unfiltered ( $\mu \mathrm{g} / \mathrm{L})$ & 101 & 10,000 & 117 & 646 & 488 \\
\hline
\end{tabular}


Table 20. Statistical summary of water-quality data for the upper Clark Fork basin, Montana, March 1985 through September 2005. -Continued

[Abbreviations: $\mathrm{ft}^{3} / \mathrm{s}$, cubic feet per second; ${ }^{\circ} \mathrm{C}$, degrees Celsius; E, estimated; $\mu \mathrm{g} / \mathrm{L}$, micrograms per liter; $\mu \mathrm{S} / \mathrm{cm}, \mathrm{microsiemens}$ per centimeter at $25^{\circ} \mathrm{C}$; $\mathrm{mg} / \mathrm{L}$, milligrams per liter; mm, millimeter; ton/d, tons per day. Symbols: <, less than laboratory reporting level ${ }^{1}$; --, indicates insufficient data greater than the laboratory reporting level to compute statistic]

\begin{tabular}{|c|c|c|c|c|c|}
\hline Property or constituent and reporting unit & $\begin{array}{l}\text { Number of } \\
\text { samples }\end{array}$ & Maximum & Minimum & Mean & Median \\
\hline \multicolumn{6}{|c|}{$\begin{array}{l}\text { 12323670-Mill Creek near Anaconda } \\
\text { Period of record for water-quality data: December 2004-September } 2005\end{array}$} \\
\hline Streamflow, instantaneous $\left(\mathrm{ft}^{3} / \mathrm{s}\right)$ & 8 & 165 & 7.4 & 63 & 20 \\
\hline pH, onsite (standard units) & 8 & 8.6 & 7.7 & 8.2 & 8.2 \\
\hline Specific conductance, onsite $(\mu \mathrm{S} / \mathrm{cm})$ & 8 & 195 & 56 & 130 & 145 \\
\hline Temperature, water $\left({ }^{\circ} \mathrm{C}\right)$ & 8 & 15.0 & 3.0 & 7.8 & 7.0 \\
\hline Hardness, filtered (mg/L as $\mathrm{CaCO}_{3}$ ) & 8 & 92 & 24 & 58 & 65 \\
\hline Calcium, filtered $(\mathrm{mg} / \mathrm{L})$ & 8 & 24.4 & 7.12 & 16.1 & 18.0 \\
\hline Magnesium, filtered (mg/L) & 8 & 7.58 & 1.45 & 4.41 & 4.84 \\
\hline Arsenic, filtered $(\mu \mathrm{g} / \mathrm{L})$ & 8 & 23.4 & 7.3 & 14.1 & 11.5 \\
\hline Arsenic, unfiltered $(\mu \mathrm{g} / \mathrm{L})$ & 8 & 26 & 9 & 16 & 14 \\
\hline Cadmium, filtered $(\mu \mathrm{g} / \mathrm{L})$ & 8 & .11 & .04 & .06 & .04 \\
\hline Cadmium, unfiltered $(\mu \mathrm{g} / \mathrm{L})$ & 8 & .18 & .05 & .09 & .08 \\
\hline Copper, filtered $(\mu \mathrm{g} / \mathrm{L})$ & 8 & 4.5 & 1.0 & 2.3 & 2.0 \\
\hline Copper, unfiltered $(\mu \mathrm{g} / \mathrm{L})$ & 8 & 10.3 & 1.3 & 4.6 & 3.6 \\
\hline Iron, filtered $(\mu \mathrm{g} / \mathrm{L})$ & 8 & 89 & 26 & 50 & 42 \\
\hline Iron, unfiltered $(\mu \mathrm{g} / \mathrm{L})$ & 8 & 620 & 90 & 255 & 170 \\
\hline Lead, filtered $(\mu \mathrm{g} / \mathrm{L})$ & 8 & .24 & .05 & .14 & .14 \\
\hline Lead, unfiltered $(\mu \mathrm{g} / \mathrm{L})$ & 8 & 3.12 & .27 & 1.07 & .58 \\
\hline Manganese, filtered ( $\mu \mathrm{g} / \mathrm{L})$ & 8 & 8.9 & 3.6 & 6.2 & 6.2 \\
\hline Manganese, unfiltered $(\mu \mathrm{g} / \mathrm{L})$ & 8 & 37 & 7 & 18 & 15 \\
\hline Zinc, filtered ( $\mu \mathrm{g} / \mathrm{L})$ & 8 & 2.4 & .8 & 1.5 & 1.2 \\
\hline Zinc, unfiltered $(\mu \mathrm{g} / \mathrm{L})$ & 8 & 8 & 1 & 4 & 2 \\
\hline Sediment, suspended (percent finer than $0.062 \mathrm{~mm}$ ) & 8 & 81 & 51 & 70 & 75 \\
\hline Sediment, suspended concentration (mg/L) & 8 & 29 & 1 & 9 & 2 \\
\hline Sediment, suspended discharge (ton/d) & 8 & 13 & .03 & 3.1 & .12 \\
\hline
\end{tabular}


Table 20. Statistical summary of water-quality data for the upper Clark Fork basin, Montana, March 1985 through September 2005. -Continued

[Abbreviations: $\mathrm{ft}^{3} / \mathrm{s}$, cubic feet per second; ${ }^{\circ} \mathrm{C}$, degrees Celsius; E, estimated; $\mu \mathrm{g} / \mathrm{L}$, micrograms per liter; $\mu \mathrm{S} / \mathrm{cm}, \mathrm{microsiemens} \mathrm{per} \mathrm{centimeter} \mathrm{at} 25^{\circ} \mathrm{C}$; $\mathrm{mg} / \mathrm{L}$, milligrams per liter; mm, millimeter; ton/d, tons per day. Symbols: <, less than laboratory reporting level ${ }^{1}$; --, indicates insufficient data greater than the laboratory reporting level to compute statistic]

\begin{tabular}{|c|c|c|c|c|c|}
\hline Property or constituent and reporting unit & $\begin{array}{l}\text { Number of } \\
\text { samples }\end{array}$ & Maximum & Minimum & Mean & Median \\
\hline \multicolumn{6}{|c|}{$\begin{array}{l}12323700 \text {-Mill Creek at Opportunity } \\
\text { Period of record for water-quality data: March 2003-September } 2005\end{array}$} \\
\hline Streamflow, instantaneous $\left(\mathrm{ft}^{3} / \mathrm{s}\right)$ & 24 & 261 & 0.43 & 37 & 5.0 \\
\hline pH, onsite (standard units) & 24 & 8.2 & 7.8 & 8.0 & 8.0 \\
\hline Hardness, filtered $\left(\mathrm{mg} / \mathrm{L}\right.$ as $\left.\mathrm{CaCO}_{3}\right)$ & 24 & 100 & 24 & 64 & 69 \\
\hline Calcium, filtered (mg/L) & 24 & 27.9 & 7.01 & 17.9 & 19.3 \\
\hline Magnesium, filtered (mg/L) & 24 & 7.68 & 1.56 & 4.62 & 4.84 \\
\hline Arsenic, filtered $(\mu \mathrm{g} / \mathrm{L})$ & 24 & 37.0 & 9.0 & 23.3 & 21.8 \\
\hline Copper, filtered $(\mu \mathrm{g} / \mathrm{L})$ & 24 & 6.1 & 1.2 & 3.2 & 3.0 \\
\hline Copper, unfiltered $(\mu \mathrm{g} / \mathrm{L})$ & 24 & 38.8 & 2.2 & 8.0 & 4.4 \\
\hline Iron, filtered $(\mu \mathrm{g} / \mathrm{L})$ & 24 & 90 & 17 & 52 & 49 \\
\hline Iron, unfiltered $(\mu \mathrm{g} / \mathrm{L})$ & 24 & 1,960 & 70 & 359 & 135 \\
\hline Lead, filtered $(\mu \mathrm{g} / \mathrm{L})$ & 24 & .32 & .04 & .16 & .14 \\
\hline Lead, unfiltered $(\mu \mathrm{g} / \mathrm{L})$ & 24 & 12.7 & .08 & 1.90 & .42 \\
\hline Manganese, filtered $(\mu \mathrm{g} / \mathrm{L})$ & 24 & 32.8 & 3.6 & 10.2 & 7.6 \\
\hline Manganese, unfiltered $(\mu \mathrm{g} / \mathrm{L})$ & 24 & 113 & 6 & 24 & 14 \\
\hline
\end{tabular}


Table 20. Statistical summary of water-quality data for the upper Clark Fork basin, Montana, March 1985 through September 2005. -Continued

[Abbreviations: $\mathrm{ft}^{3} / \mathrm{s}$, cubic feet per second; ${ }^{\circ} \mathrm{C}$, degrees Celsius; E, estimated; $\mu \mathrm{g} / \mathrm{L}$, micrograms per liter; $\mu \mathrm{S} / \mathrm{cm}, \mathrm{microsiemens}$ per centimeter at $25^{\circ} \mathrm{C}$; $\mathrm{mg} / \mathrm{L}$, milligrams per liter; mm, millimeter; ton/d, tons per day. Symbols: <, less than laboratory reporting level ${ }^{1}$; --, indicates insufficient data greater than the laboratory reporting level to compute statistic]

\begin{tabular}{|c|c|c|c|c|c|}
\hline Property or constituent and reporting unit & $\begin{array}{c}\text { Number of } \\
\text { samples }\end{array}$ & Maximum & Minimum & Mean & Median \\
\hline \multicolumn{6}{|c|}{$\begin{array}{l}12323710 \text {-Willow Creek near Anaconda } \\
\text { Period of record for water-quality data: December 2004-September } 2005\end{array}$} \\
\hline Streamflow, instantaneous $\left(\mathrm{ft}^{3} / \mathrm{s}\right)$ & 8 & 39 & 1.0 & 11 & 2.6 \\
\hline pH, onsite (standard units) & 8 & 8.2 & 7.5 & 7.8 & 7.8 \\
\hline Specific conductance, onsite $(\mu \mathrm{S} / \mathrm{cm})$ & 8 & 114 & 66 & 99 & 105 \\
\hline Temperature, water $\left({ }^{\circ} \mathrm{C}\right)$ & 8 & 13.0 & .5 & 6.0 & 5.5 \\
\hline Hardness, filtered (mg/L as $\left.\mathrm{CaCO}_{3}\right)$ & 8 & 42 & 23 & 36 & 39 \\
\hline Calcium, filtered $(\mathrm{mg} / \mathrm{L})$ & 8 & 13.9 & 7.74 & 12.3 & 13.2 \\
\hline Magnesium, filtered (mg/L) & 8 & 1.69 & .88 & 1.35 & 1.36 \\
\hline Arsenic, filtered $(\mu \mathrm{g} / \mathrm{L})$ & 8 & 24.3 & 9.9 & 15.0 & 12.8 \\
\hline Arsenic, unfiltered $(\mu \mathrm{g} / \mathrm{L})$ & 8 & 27 & 10 & 17 & 14 \\
\hline Cadmium, filtered $(\mu \mathrm{g} / \mathrm{L})$ & 8 & .05 & .02 & .03 & .03 \\
\hline Cadmium, unfiltered ( $\mu \mathrm{g} / \mathrm{L})$ & 8 & .19 & .03 & .07 & .06 \\
\hline Copper, filtered $(\mu \mathrm{g} / \mathrm{L})$ & 8 & 4.2 & 1.0 & 2.2 & 2.0 \\
\hline Copper, unfiltered $(\mu \mathrm{g} / \mathrm{L})$ & 8 & 10.5 & 1.5 & 3.7 & 3.0 \\
\hline Iron, filtered $(\mu \mathrm{g} / \mathrm{L})$ & 8 & 125 & 36 & 66 & 60 \\
\hline Iron, unfiltered $(\mu \mathrm{g} / \mathrm{L})$ & 8 & 1,260 & 90 & 314 & 180 \\
\hline Lead, filtered $(\mu \mathrm{g} / \mathrm{L})$ & 8 & .37 & .05 & .15 & .14 \\
\hline Lead, unfiltered ( $\mu \mathrm{g} / \mathrm{L})$ & 8 & 4.08 & .20 & .93 & .48 \\
\hline Manganese, filtered $(\mu \mathrm{g} / \mathrm{L})$ & 8 & 23.0 & 11.4 & 16.4 & 17.1 \\
\hline Manganese, unfiltered $(\mu \mathrm{g} / \mathrm{L})$ & 8 & 49 & 15 & 29 & 24 \\
\hline Zinc, filtered $(\mu \mathrm{g} / \mathrm{L})$ & 8 & 3.3 & 1.1 & 2.0 & 1.8 \\
\hline Zinc, unfiltered $(\mu \mathrm{g} / \mathrm{L})$ & 8 & 10 & 1 & 3 & 2 \\
\hline Sediment, suspended (percent finer than $0.062 \mathrm{~mm}$ ) & 8 & 94 & 44 & 76 & 82 \\
\hline Sediment, suspended concentration (mg/L) & 8 & 93 & 3 & 17 & 4 \\
\hline Sediment, suspended discharge (ton/d) & 8 & 9.8 & .01 & 1.4 & .04 \\
\hline
\end{tabular}


Table 20. Statistical summary of water-quality data for the upper Clark Fork basin, Montana, March 1985 through September 2005. -Continued

[Abbreviations: $\mathrm{ft}^{3} / \mathrm{s}$, cubic feet per second; ${ }^{\circ} \mathrm{C}$, degrees Celsius; E, estimated; $\mu \mathrm{g} / \mathrm{L}$, micrograms per liter; $\mu \mathrm{S} / \mathrm{cm}, \mathrm{microsiemens} \mathrm{per} \mathrm{centimeter} \mathrm{at} 25^{\circ} \mathrm{C}$; $\mathrm{mg} / \mathrm{L}$, milligrams per liter; mm, millimeter; ton/d, tons per day. Symbols: <, less than laboratory reporting level ${ }^{1}$; --, indicates insufficient data greater than the laboratory reporting level to compute statistic]

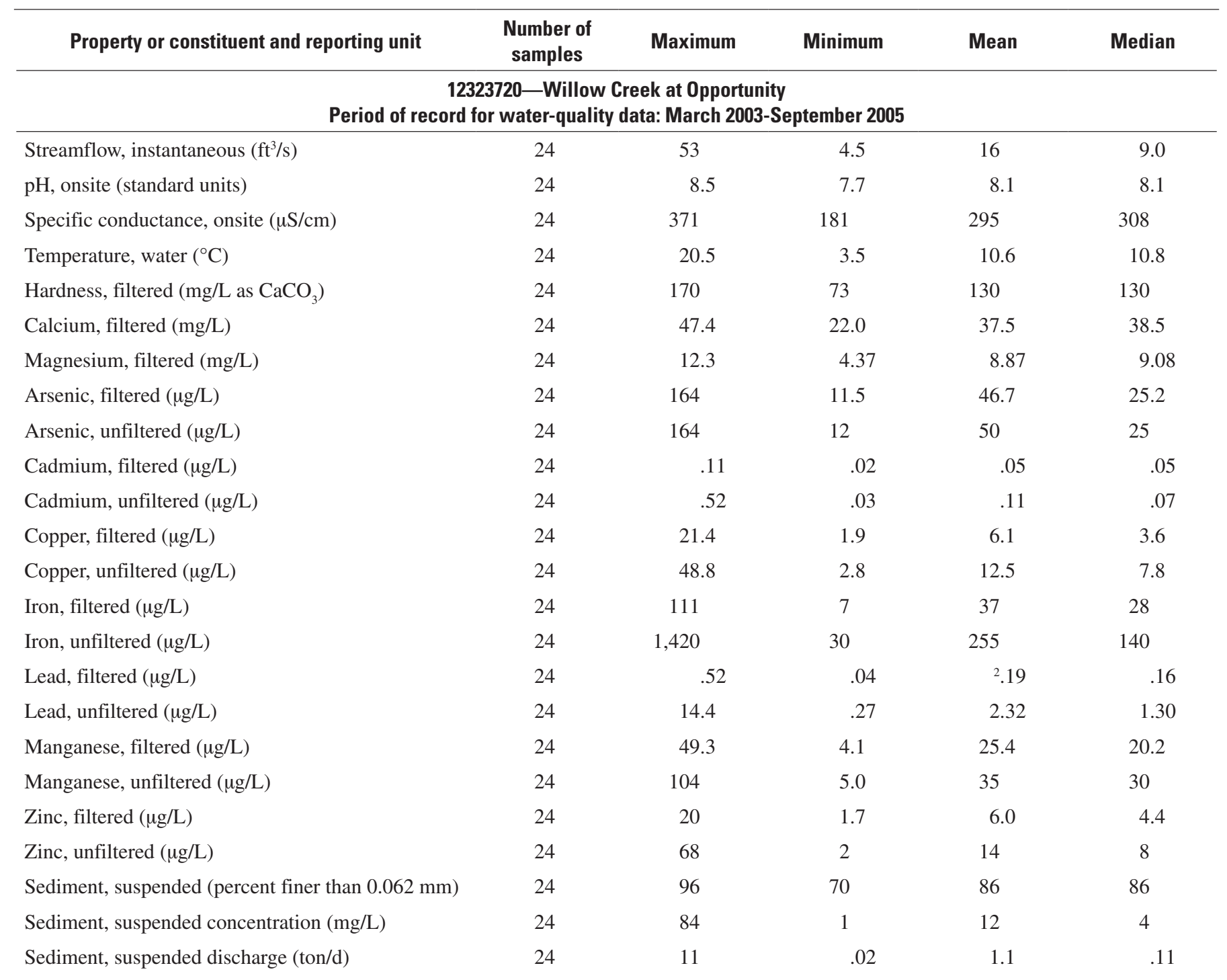


Table 20. Statistical summary of water-quality data for the upper Clark Fork basin, Montana, March 1985 through September 2005. -Continued

[Abbreviations: $\mathrm{ft}^{3} / \mathrm{s}$, cubic feet per second; ${ }^{\circ} \mathrm{C}$, degrees Celsius; E, estimated; $\mu \mathrm{g} / \mathrm{L}$, micrograms per liter; $\mu \mathrm{S} / \mathrm{cm}, \mathrm{microsiemens}$ per centimeter at $25^{\circ} \mathrm{C}$; $\mathrm{mg} / \mathrm{L}$, milligrams per liter; mm, millimeter; ton/d, tons per day. Symbols: <, less than laboratory reporting level ${ }^{1}$; --, indicates insufficient data greater than the laboratory reporting level to compute statistic]

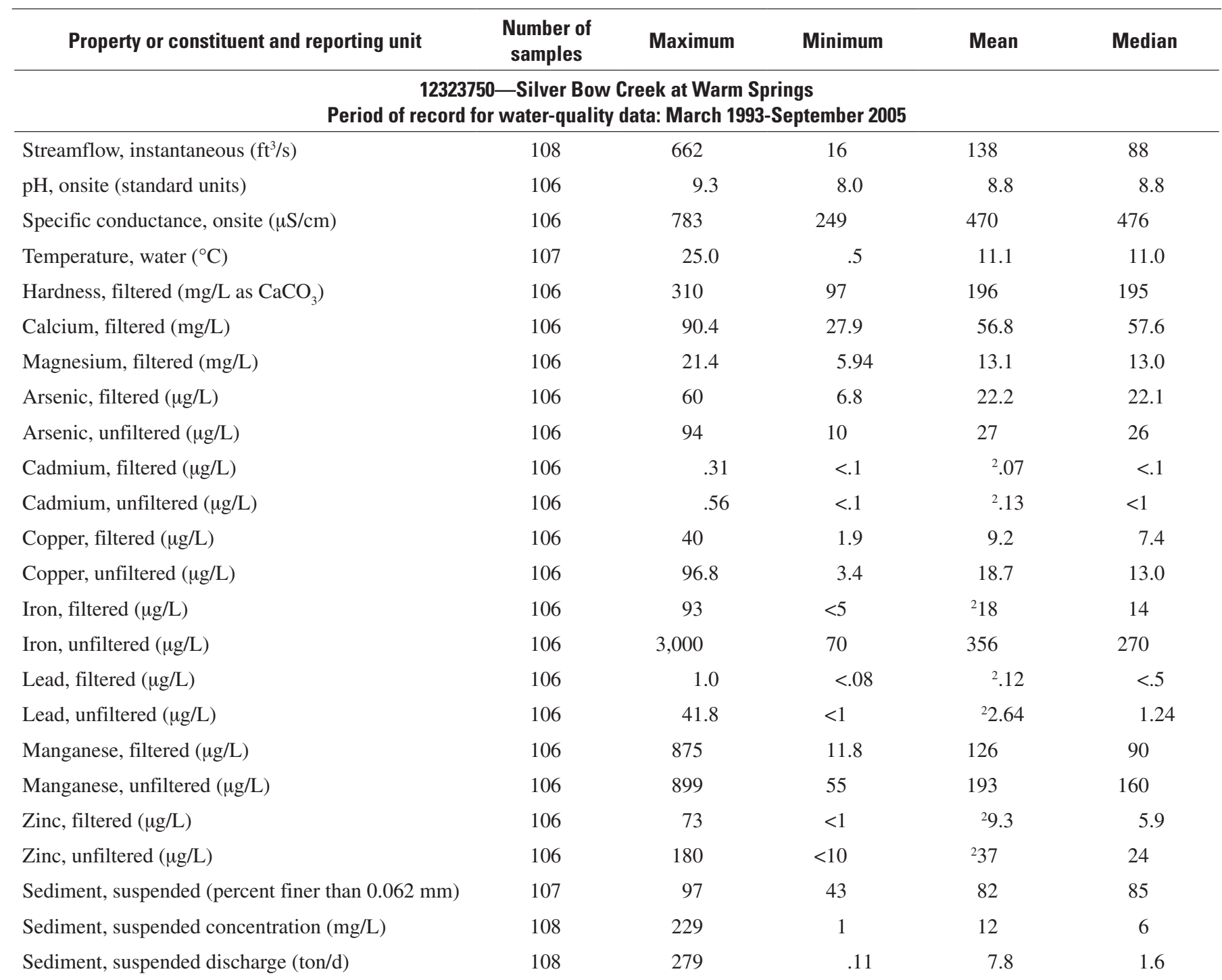


Table 20. Statistical summary of water-quality data for the upper Clark Fork basin, Montana, March 1985 through September 2005. -Continued

[Abbreviations: $\mathrm{ft}^{3} / \mathrm{s}$, cubic feet per second; ${ }^{\circ} \mathrm{C}$, degrees Celsius; E, estimated; $\mu \mathrm{g} / \mathrm{L}$, micrograms per liter; $\mu \mathrm{S} / \mathrm{cm}, \mathrm{microsiemens}$ per centimeter at $25^{\circ} \mathrm{C}$; $\mathrm{mg} / \mathrm{L}$, milligrams per liter; mm, millimeter; ton/d, tons per day. Symbols: <, less than laboratory reporting level ${ }^{1}$; --, indicates insufficient data greater than the laboratory reporting level to compute statistic]

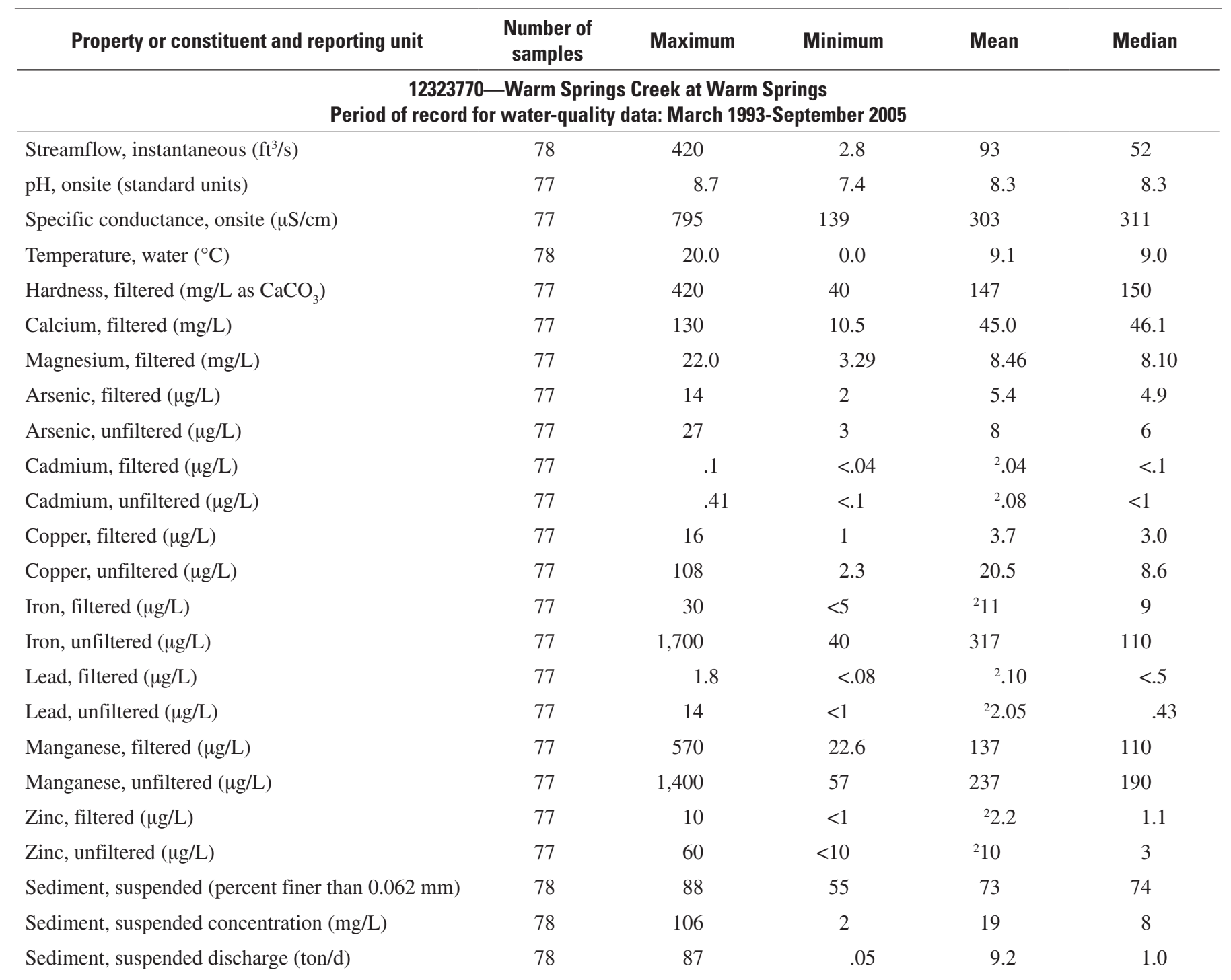


Table 20. Statistical summary of water-quality data for the upper Clark Fork basin, Montana, March 1985 through September 2005. -Continued

[Abbreviations: $\mathrm{ft}^{3} / \mathrm{s}$, cubic feet per second; ${ }^{\circ} \mathrm{C}$, degrees Celsius; $\mathrm{E}$, estimated; $\mu \mathrm{g} / \mathrm{L}$, micrograms per liter; $\mu \mathrm{S} / \mathrm{cm}, \mathrm{microsiemens} \mathrm{per} \mathrm{centimeter} \mathrm{at} 25^{\circ} \mathrm{C}$; $\mathrm{mg} / \mathrm{L}$, milligrams per liter; mm, millimeter; ton/d, tons per day. Symbols: <, less than laboratory reporting level ${ }^{1}$; --, indicates insufficient data greater than the laboratory reporting level to compute statistic]

\begin{tabular}{|c|c|c|c|c|c|}
\hline Property or constituent and reporting unit & $\begin{array}{c}\text { Number of } \\
\text { samples }\end{array}$ & Maximum & Minimum & Mean & Median \\
\hline \multicolumn{6}{|c|}{$\begin{array}{l}12323800 \text { —Clark Fork near Galen } \\
\text { Period of record for water-quality data: July 1988-September } 2005\end{array}$} \\
\hline Streamflow, instantaneous $\left(\mathrm{ft}^{3} / \mathrm{s}\right)$ & 149 & 1,050 & 14 & 208 & 124 \\
\hline pH, onsite (standard units) & 136 & 9.0 & 7.5 & 8.5 & 8.6 \\
\hline Specific conductance, onsite $(\mu \mathrm{S} / \mathrm{cm})$ & 137 & 720 & 197 & 427 & 440 \\
\hline Temperature, water $\left({ }^{\circ} \mathrm{C}\right)$ & 148 & 22.5 & 0.0 & 10.0 & 10.0 \\
\hline Hardness, filtered $\left(\mathrm{mg} / \mathrm{L}\right.$ as $\left.\mathrm{CaCO}_{3}\right)$ & 135 & 370 & 81 & 187 & 190 \\
\hline Calcium, filtered (mg/L) & 135 & 110 & 24.2 & 55.2 & 57.5 \\
\hline Magnesium, filtered (mg/L) & 135 & 22.0 & 5.08 & 11.9 & 12.1 \\
\hline Arsenic, filtered $(\mu \mathrm{g} / \mathrm{L})$ & 135 & 53 & 4 & 15.2 & 13.3 \\
\hline Arsenic, unfiltered $(\mu \mathrm{g} / \mathrm{L})$ & 135 & 78 & 3 & 20 & 17 \\
\hline Cadmium, filtered $(\mu \mathrm{g} / \mathrm{L})$ & 135 & 1 & $<.1$ & ${ }^{2} .07$ & $<1$ \\
\hline Cadmium, unfiltered $(\mu \mathrm{g} / \mathrm{L})$ & 135 & 3 & $<.1$ & ${ }^{2} .21$ & $<1$ \\
\hline Copper, filtered $(\mu \mathrm{g} / \mathrm{L})$ & 135 & 50 & 2.3 & 9.0 & 7.0 \\
\hline Copper, unfiltered $(\mu \mathrm{g} / \mathrm{L})$ & 134 & 240 & 4.8 & 30.4 & 17.0 \\
\hline Iron, filtered $(\mu \mathrm{g} / \mathrm{L})$ & 135 & 110 & $<3$ & ${ }^{2} 16$ & 11 \\
\hline Iron, unfiltered $(\mu \mathrm{g} / \mathrm{L})$ & 135 & 9,200 & 60 & 523 & 270 \\
\hline Lead, filtered $(\mu \mathrm{g} / \mathrm{L})$ & 135 & 3 & $<.08$ & ${ }^{2} .18$ & $<1$ \\
\hline Lead, unfiltered $(\mu \mathrm{g} / \mathrm{L})$ & 135 & 31.0 & $<1$ & ${ }^{2} 3.76$ & 2.00 \\
\hline Manganese, filtered $(\mu \mathrm{g} / \mathrm{L})$ & 135 & 460 & 25.2 & 115 & 82.7 \\
\hline Manganese, unfiltered $(\mu \mathrm{g} / \mathrm{L})$ & 135 & 1,400 & 47 & 248 & 193 \\
\hline Zinc, filtered ( $\mu \mathrm{g} / \mathrm{L})$ & 135 & 110 & $<1$ & ${ }^{2} 11.2$ & 6.0 \\
\hline Zinc, unfiltered $(\mu \mathrm{g} / \mathrm{L})$ & 135 & 360 & $<10$ & ${ }^{2} 44$ & 29 \\
\hline Sediment, suspended (percent finer than $0.062 \mathrm{~mm}$ ) & 148 & 97 & 41 & 78 & 78 \\
\hline Sediment, suspended concentration (mg/L) & 149 & 338 & 2 & 19 & 8 \\
\hline Sediment, suspended discharge (ton/d) & 149 & 459 & .12 & 22 & 2.3 \\
\hline
\end{tabular}




\section{Water-Quality, Bed-Sediment, Biological Data, and Statistical Summaries, Upper Clark Fork Basin, Montana}

Table 20. Statistical summary of water-quality data for the upper Clark Fork basin, Montana, March 1985 through September 2005. -Continued

[Abbreviations: $\mathrm{ft}^{3} / \mathrm{s}$, cubic feet per second; ${ }^{\circ} \mathrm{C}$, degrees Celsius; E, estimated; $\mu \mathrm{g} / \mathrm{L}$, micrograms per liter; $\mu \mathrm{S} / \mathrm{cm}, \mathrm{microsiemens} \mathrm{per} \mathrm{centimeter} \mathrm{at} 25^{\circ} \mathrm{C}$; $\mathrm{mg} / \mathrm{L}$, milligrams per liter; mm, millimeter; ton/d, tons per day. Symbols: <, less than laboratory reporting level ${ }^{1}$; --, indicates insufficient data greater than the laboratory reporting level to compute statistic]

\begin{tabular}{|c|c|c|c|c|c|}
\hline Property or constituent and reporting unit & $\begin{array}{l}\text { Number of } \\
\text { samples }\end{array}$ & Maximum & Minimum & Mean & Median \\
\hline \multicolumn{6}{|c|}{$\begin{array}{c}\text { 12323840—Lost Creek near Anaconda } \\
\text { Period of record for water-quality data: December 2004-September } 2005\end{array}$} \\
\hline Streamflow, instantaneous $\left(\mathrm{ft}^{3} / \mathrm{s}\right)$ & 8 & 17 & .97 & 7.1 & 4.2 \\
\hline $\mathrm{pH}$, onsite (standard units) & 8 & 8.6 & 7.4 & 8.2 & 8.4 \\
\hline Specific conductance, onsite $(\mu \mathrm{S} / \mathrm{cm})$ & 8 & 227 & 163 & 202 & 216 \\
\hline Temperature, water $\left({ }^{\circ} \mathrm{C}\right)$ & 8 & 14.5 & 3.0 & 8.5 & 7.8 \\
\hline Hardness, filtered $\left(\mathrm{mg} / \mathrm{L}\right.$ as $\mathrm{CaCO}_{3}$ ) & 8 & 110 & 50 & 94 & 100 \\
\hline Calcium, filtered (mg/L) & 8 & 35.7 & 15.7 & 28.9 & 30.6 \\
\hline Magnesium, filtered (mg/L) & 8 & 6.79 & 2.71 & 5.50 & 6.14 \\
\hline Arsenic, filtered $(\mu \mathrm{g} / \mathrm{L})$ & 8 & 156 & 2.5 & 24.6 & 6.8 \\
\hline Arsenic, unfiltered $(\mu \mathrm{g} / \mathrm{L})$ & 8 & 3,860 & 2 & 488 & 8 \\
\hline Cadmium, filtered $(\mu \mathrm{g} / \mathrm{L})$ & 8 & .90 & .02 & .15 & .04 \\
\hline Cadmium, unfiltered $(\mu \mathrm{g} / \mathrm{L})$ & 8 & 147 & .02 & 18.4 & .06 \\
\hline Copper, filtered $(\mu \mathrm{g} / \mathrm{L})$ & 8 & 90.5 & 1.2 & 13.7 & 3.4 \\
\hline Copper, unfiltered $(\mu \mathrm{g} / \mathrm{L})$ & 8 & 29,100 & 1.7 & 3,640 & 6.4 \\
\hline Iron, filtered $(\mu \mathrm{g} / \mathrm{L})$ & 8 & 25 & $<6$ & 29 & 4 \\
\hline Iron, unfiltered $(\mu \mathrm{g} / \mathrm{L})$ & 8 & 99,700 & 20 & 12,600 & 110 \\
\hline Lead, filtered $(\mu \mathrm{g} / \mathrm{L})$ & 8 & .18 & $<.08$ & -- & $<.08$ \\
\hline Lead, unfiltered $(\mu \mathrm{g} / \mathrm{L})$ & 8 & 1,290 & .13 & 162 & .54 \\
\hline Manganese, filtered ( $\mu \mathrm{g} / \mathrm{L})$ & 8 & 42.4 & .5 & 6.5 & 1.6 \\
\hline Manganese, unfiltered ( $\mu \mathrm{g} / \mathrm{L})$ & 8 & 8,830 & 1 & 1,110 & 4 \\
\hline Zinc, filtered $(\mu \mathrm{g} / \mathrm{L})$ & 8 & 30.0 & 1.0 & 4.9 & 1.2 \\
\hline Zinc, unfiltered $(\mu \mathrm{g} / \mathrm{L})$ & 8 & 7,780 & 2 & 976 & 4 \\
\hline Sediment, suspended (percent finer than $0.062 \mathrm{~mm}$ ) & 8 & 97 & 30 & 70 & 76 \\
\hline Sediment, suspended concentration (mg/L) & 8 & 58,900 & 1 & 7,370 & 10 \\
\hline Sediment, suspended discharge (ton/d) & 8 & 1,320 & $<.1$ & ${ }^{2} 165$ & .08 \\
\hline
\end{tabular}


Table 20. Statistical summary of water-quality data for the upper Clark Fork basin, Montana, March 1985 through September 2005. -Continued

[Abbreviations: $\mathrm{ft}^{3} / \mathrm{s}$, cubic feet per second; ${ }^{\circ} \mathrm{C}$, degrees Celsius; E, estimated; $\mu \mathrm{g} / \mathrm{L}$, micrograms per liter; $\mu \mathrm{S} / \mathrm{cm}, \mathrm{microsiemens}$ per centimeter at $25^{\circ} \mathrm{C}$; $\mathrm{mg} / \mathrm{L}$, milligrams per liter; mm, millimeter; ton/d, tons per day. Symbols: <, less than laboratory reporting level ${ }^{1}$; --, indicates insufficient data greater than the laboratory reporting level to compute statistic]

\begin{tabular}{|c|c|c|c|c|c|}
\hline Property or constituent and reporting unit & $\begin{array}{c}\text { Number of } \\
\text { samples }\end{array}$ & Maximum & Minimum & Mean & Median \\
\hline \multicolumn{6}{|c|}{$\begin{array}{l}\text { 12323850-Lost Creek near Galen } \\
\text { Period of record for water-quality data: March 2003-September } 2005\end{array}$} \\
\hline Streamflow, instantaneous $\left(\mathrm{ft}^{3} / \mathrm{s}\right)$ & 24 & 59 & 1.3 & 19 & 12 \\
\hline $\mathrm{pH}$, onsite (standard units) & 24 & 8.7 & 8.0 & 8.5 & 8.5 \\
\hline Specific conductance, onsite $(\mu \mathrm{S} / \mathrm{cm})$ & 24 & 934 & 540 & 653 & 630 \\
\hline Temperature, water $\left({ }^{\circ} \mathrm{C}\right)$ & 24 & 26.5 & 1.5 & 13.0 & 12.2 \\
\hline Hardness, filtered (mg/L as $\left.\mathrm{CaCO}_{3}\right)$ & 24 & 450 & 200 & 307 & 300 \\
\hline Calcium, filtered $(\mathrm{mg} / \mathrm{L})$ & 24 & 122 & 48.5 & 85.6 & 86.6 \\
\hline Magnesium, filtered (mg/L) & 24 & 35.7 & 18.0 & 22.7 & 21.2 \\
\hline Arsenic, filtered $(\mu \mathrm{g} / \mathrm{L})$ & 24 & 41.8 & 6.6 & 15.2 & 12.9 \\
\hline Arsenic, unfiltered $(\mu \mathrm{g} / \mathrm{L})$ & 24 & 43 & 6 & 15 & 14 \\
\hline Cadmium, filtered $(\mu \mathrm{g} / \mathrm{L})$ & 24 & .05 & $<.04$ & ${ }^{2} .03$ & .03 \\
\hline Cadmium, unfiltered ( $\mu \mathrm{g} / \mathrm{L})$ & 24 & .11 & .02 & .05 & .04 \\
\hline Copper, filtered $(\mu \mathrm{g} / \mathrm{L})$ & 24 & 6.7 & 1.5 & 3.2 & 2.9 \\
\hline Copper, unfiltered $(\mu \mathrm{g} / \mathrm{L})$ & 24 & 22.5 & 3.9 & 7.5 & 6.2 \\
\hline Iron, filtered $(\mu \mathrm{g} / \mathrm{L})$ & 24 & 61 & $<6$ & ${ }^{2} 11$ & 7 \\
\hline Iron, unfiltered $(\mu \mathrm{g} / \mathrm{L})$ & 24 & 280 & 10 & 98 & 75 \\
\hline Lead, filtered $(\mu \mathrm{g} / \mathrm{L})$ & 24 & .33 & $<.08$ & ${ }^{2} .08$ & $<.08$ \\
\hline Lead, unfiltered ( $\mu \mathrm{g} / \mathrm{L})$ & 24 & 1.30 & .04 & .39 & .31 \\
\hline Manganese, filtered $(\mu \mathrm{g} / \mathrm{L})$ & 24 & 39.5 & 1.9 & 11.6 & 9.4 \\
\hline Manganese, unfiltered $(\mu \mathrm{g} / \mathrm{L})$ & 24 & 45 & 2 & 15 & 13 \\
\hline Zinc, filtered $(\mu \mathrm{g} / \mathrm{L})$ & 24 & 3.8 & $<1$ & ${ }^{2} 1.6$ & 1.2 \\
\hline Zinc, unfiltered $(\mu \mathrm{g} / \mathrm{L})$ & 24 & 9 & $<2$ & ${ }^{2} 3$ & 3 \\
\hline Sediment, suspended (percent finer than $0.062 \mathrm{~mm}$ ) & 24 & 86 & 18 & 55 & 60 \\
\hline Sediment, suspended concentration (mg/L) & 24 & 34 & 2 & 14 & 14 \\
\hline Sediment, suspended discharge (ton/d) & 24 & 3.8 & .01 & .89 & .32 \\
\hline
\end{tabular}




\section{Water-Quality, Bed-Sediment, Biological Data, and Statistical Summaries, Upper Clark Fork Basin, Montana}

Table 20. Statistical summary of water-quality data for the upper Clark Fork basin, Montana, March 1985 through September 2005. -Continued

[Abbreviations: $\mathrm{ft}^{3} / \mathrm{s}$, cubic feet per second; ${ }^{\circ} \mathrm{C}$, degrees Celsius; E, estimated; $\mu \mathrm{g} / \mathrm{L}$, micrograms per liter; $\mu \mathrm{S} / \mathrm{cm}, \mathrm{microsiemens} \mathrm{per} \mathrm{centimeter} \mathrm{at} 25^{\circ} \mathrm{C}$; $\mathrm{mg} / \mathrm{L}$, milligrams per liter; mm, millimeter; ton/d, tons per day. Symbols: <, less than laboratory reporting level ${ }^{1}$; --, indicates insufficient data greater than the laboratory reporting level to compute statistic]

\begin{tabular}{|c|c|c|c|c|c|}
\hline Property or constituent and reporting unit & $\begin{array}{l}\text { Number of } \\
\text { samples }\end{array}$ & Maximum & Minimum & Mean & Median \\
\hline \multicolumn{6}{|c|}{$\begin{array}{l}12324200 \text {-Clark Fork at Deer Lodge } \\
\text { Period of record for water-quality data: March 1985-September } 2005\end{array}$} \\
\hline Streamflow, instantaneous $\left(\mathrm{ft}^{3} / \mathrm{s}\right)$ & 201 & 1,920 & 23 & 290 & 215 \\
\hline pH, onsite (standard units) & 149 & 8.9 & 7.4 & 8.3 & 8.3 \\
\hline Hardness, filtered $\left(\mathrm{mg} / \mathrm{L}\right.$ as $\left.\mathrm{CaCO}_{3}\right)$ & 141 & 280 & 95 & 205 & 220 \\
\hline Calcium, filtered $(\mathrm{mg} / \mathrm{L})$ & 141 & 82.0 & 28.2 & 60.5 & 64.0 \\
\hline Magnesium, filtered (mg/L) & 141 & 19 & 5.9 & 13.1 & 13.8 \\
\hline Arsenic, filtered $(\mu \mathrm{g} / \mathrm{L})$ & 151 & 39 & 6 & 14.4 & 13.0 \\
\hline Copper, filtered $(\mu \mathrm{g} / \mathrm{L})$ & 151 & 120 & 3.2 & 11.6 & 9.0 \\
\hline Copper, unfiltered $(\mu \mathrm{g} / \mathrm{L})$ & 150 & 1,500 & 8.2 & 89.8 & 38.2 \\
\hline Iron, filtered $(\mu \mathrm{g} / \mathrm{L})$ & 151 & 190 & $<3$ & ${ }^{2} 15$ & 9 \\
\hline Iron, unfiltered $(\mu \mathrm{g} / \mathrm{L})$ & 151 & 29,000 & 30 & 1,670 & 580 \\
\hline Lead, filtered $(\mu \mathrm{g} / \mathrm{L})$ & 151 & 6 & $<.08$ & ${ }^{2} .38$ & $<1$ \\
\hline Lead, unfiltered $(\mu \mathrm{g} / \mathrm{L})$ & 151 & 200 & $<1$ & ${ }^{2} 11.7$ & 4.08 \\
\hline Manganese, filtered ( $\mu \mathrm{g} / \mathrm{L})$ & 151 & 400 & 1 & 43.1 & 33.9 \\
\hline Manganese, unfiltered ( $\mu \mathrm{g} / \mathrm{L})$ & 151 & 4,600 & 12 & 264 & 150 \\
\hline
\end{tabular}


Table 20. Statistical summary of water-quality data for the upper Clark Fork basin, Montana, March 1985 through September 2005. -Continued

[Abbreviations: $\mathrm{ft}^{3} / \mathrm{s}$, cubic feet per second; ${ }^{\circ} \mathrm{C}$, degrees Celsius; E, estimated; $\mu \mathrm{g} / \mathrm{L}$, micrograms per liter; $\mu \mathrm{S} / \mathrm{cm}, \mathrm{microsiemens}$ per centimeter at $25^{\circ} \mathrm{C}$; $\mathrm{mg} / \mathrm{L}$, milligrams per liter; mm, millimeter; ton/d, tons per day. Symbols: <, less than laboratory reporting level ${ }^{1}$; --, indicates insufficient data greater than the laboratory reporting level to compute statistic]

\begin{tabular}{|c|c|c|c|c|c|}
\hline Property or constituent and reporting unit & $\begin{array}{l}\text { Number of } \\
\text { samples }\end{array}$ & Maximum & Minimum & Mean & Median \\
\hline \multicolumn{6}{|c|}{$\begin{array}{c}12324680 \text { - Clark Fork at Goldcreek } \\
\text { Period of record for water-quality data: March 1993-September } 2005\end{array}$} \\
\hline Streamflow, instantaneous $\left(\mathrm{ft}^{3} / \mathrm{s}\right)$ & 107 & 3,920 & 87 & 743 & 507 \\
\hline $\mathrm{pH}$, onsite (standard units) & 106 & 8.8 & 7.9 & 8.4 & 8.3 \\
\hline Specific conductance, onsite $(\mu \mathrm{S} / \mathrm{cm})$ & 106 & 510 & 206 & 373 & 394 \\
\hline Temperature, water $\left({ }^{\circ} \mathrm{C}\right)$ & 106 & 23.0 & 0.0 & 9.8 & 10.0 \\
\hline Hardness, filtered (mg/L as $\left.\mathrm{CaCO}_{3}\right)$ & 106 & 230 & 86 & 164 & 170 \\
\hline Calcium, filtered (mg/L) & 106 & 68.0 & 25.9 & 48.4 & 51.2 \\
\hline Magnesium, filtered (mg/L) & 106 & 15.0 & 5.15 & 10.5 & 11.0 \\
\hline Arsenic, filtered $(\mu \mathrm{g} / \mathrm{L})$ & 106 & 20 & 5.8 & 10.1 & 10.0 \\
\hline Arsenic, unfiltered $(\mu \mathrm{g} / \mathrm{L})$ & 106 & 75 & 7 & 15 & 12 \\
\hline Cadmium, filtered $(\mu \mathrm{g} / \mathrm{L})$ & 106 & .2 & $<.04$ & ${ }^{2} .04$ & $<.1$ \\
\hline Cadmium, unfiltered $(\mu \mathrm{g} / \mathrm{L})$ & 106 & 2 & $<.1$ & ${ }^{2} .18$ & $<1$ \\
\hline Copper, filtered $(\mu \mathrm{g} / \mathrm{L})$ & 105 & 36 & 2.1 & 6.9 & 5.6 \\
\hline Copper, unfiltered $(\mu \mathrm{g} / \mathrm{L})$ & 105 & 440 & 5.2 & 43.1 & 25.0 \\
\hline Iron, filtered $(\mu \mathrm{g} / \mathrm{L})$ & 106 & 100 & $<3$ & ${ }^{2} 19$ & 12 \\
\hline Iron, unfiltered $(\mu \mathrm{g} / \mathrm{L})$ & 106 & 12,000 & 30 & 929 & 460 \\
\hline Lead, filtered $(\mu \mathrm{g} / \mathrm{L})$ & 105 & .8 & $<.08$ & ${ }^{2} .11$ & $<.5$ \\
\hline Lead, unfiltered $(\mu \mathrm{g} / \mathrm{L})$ & 105 & 73 & $<1$ & ${ }^{2} 6.04$ & 3.00 \\
\hline Manganese, filtered $(\mu \mathrm{g} / \mathrm{L})$ & 106 & 57.3 & 4.0 & 19.6 & 17.8 \\
\hline Manganese, unfiltered $(\mu \mathrm{g} / \mathrm{L})$ & 106 & 1,100 & 10 & 132 & 90 \\
\hline Zinc, filtered $(\mu \mathrm{g} / \mathrm{L})$ & 106 & 26 & $<1$ & ${ }^{2} 6.2$ & 4.0 \\
\hline Zinc, unfiltered ( $\mu \mathrm{g} / \mathrm{L})$ & 106 & 510 & 2 & 49 & 30 \\
\hline Sediment, suspended (percent finer than $0.062 \mathrm{~mm}$ ) & 107 & 94 & 43 & 76 & 78 \\
\hline Sediment, suspended concentration (mg/L) & 107 & 752 & 2 & 54 & 22 \\
\hline Sediment, suspended discharge (ton/d) & 107 & 7,960 & .94 & 238 & 33 \\
\hline
\end{tabular}


Table 20. Statistical summary of water-quality data for the upper Clark Fork basin, Montana, March 1985 through September 2005. -Continued

[Abbreviations: $\mathrm{ft}^{3} / \mathrm{s}$, cubic feet per second; ${ }^{\circ} \mathrm{C}$, degrees Celsius; E, estimated; $\mu \mathrm{g} / \mathrm{L}$, micrograms per liter; $\mu \mathrm{S} / \mathrm{cm}, \mathrm{microsiemens}$ per centimeter at $25^{\circ} \mathrm{C}$; $\mathrm{mg} / \mathrm{L}$, milligrams per liter; mm, millimeter; ton/d, tons per day. Symbols: <, less than laboratory reporting level ${ }^{1}$; --, indicates insufficient data greater than the laboratory reporting level to compute statistic]

\begin{tabular}{|c|c|c|c|c|c|}
\hline Property or constituent and reporting unit & $\begin{array}{l}\text { Number of } \\
\text { samples }\end{array}$ & Maximum & Minimum & Mean & Median \\
\hline \multicolumn{6}{|c|}{$\begin{array}{l}12331800 \text { —Clark Fork near Drummond } \\
\text { Period of record for water-quality data: March 1993-September } 2005\end{array}$} \\
\hline Streamflow, instantaneous $\left(\mathrm{ft}^{3} / \mathrm{s}\right)$ & 107 & 3,860 & 149 & 1,030 & 755 \\
\hline pH, onsite (standard units) & 106 & 8.6 & 7.8 & 8.3 & 8.3 \\
\hline Hardness, filtered $\left(\mathrm{mg} / \mathrm{L}\right.$ as $\left.\mathrm{CaCO}_{3}\right)$ & 106 & 300 & 74 & 186 & 195 \\
\hline Calcium, filtered $(\mathrm{mg} / \mathrm{L})$ & 106 & 83 & 21 & 53.4 & 55.2 \\
\hline Magnesium, filtered (mg/L) & 106 & 22 & 5.2 & 12.8 & 13.1 \\
\hline Arsenic, filtered $(\mu \mathrm{g} / \mathrm{L})$ & 106 & 20 & 6.6 & 10.6 & 10.0 \\
\hline Copper, filtered $(\mu \mathrm{g} / \mathrm{L})$ & 104 & 21 & 1 & 6.6 & 5.0 \\
\hline Copper, unfiltered $(\mu \mathrm{g} / \mathrm{L})$ & 104 & 360 & 4.6 & 46.2 & 23.0 \\
\hline Iron, filtered $(\mu \mathrm{g} / \mathrm{L})$ & 106 & 150 & $<3$ & ${ }^{2} 19$ & 8 \\
\hline Iron, unfiltered $(\mu \mathrm{g} / \mathrm{L})$ & 105 & 8,800 & 20 & 1,060 & 470 \\
\hline Lead, filtered $(\mu \mathrm{g} / \mathrm{L})$ & 102 & 1.2 & $<.08$ & ${ }^{2} .17$ & $<.60$ \\
\hline Lead, unfiltered $(\mu \mathrm{g} / \mathrm{L})$ & 102 & 56 & $<1$ & ${ }^{2} 8.32$ & 3.50 \\
\hline Manganese, filtered ( $\mu \mathrm{g} / \mathrm{L})$ & 106 & 60.7 & 4.5 & 17.1 & 15.0 \\
\hline Manganese, unfiltered ( $\mu \mathrm{g} / \mathrm{L})$ & 106 & 880 & 8 & 155 & 97.5 \\
\hline
\end{tabular}


Table 20. Statistical summary of water-quality data for the upper Clark Fork basin, Montana, March 1985 through September 2005. -Continued

[Abbreviations: $\mathrm{ft}^{3} / \mathrm{s}$, cubic feet per second; ${ }^{\circ} \mathrm{C}$, degrees Celsius; E, estimated; $\mu \mathrm{g} / \mathrm{L}$, micrograms per liter; $\mu \mathrm{S} / \mathrm{cm}, \mathrm{microsiemens}$ per centimeter at $25^{\circ} \mathrm{C} ; \mathrm{mg} / \mathrm{L}$, milligrams per liter; mm, millimeter; ton/d, tons per day. Symbols: <, less than laboratory reporting level ${ }^{1}$; --, indicates insufficient data greater than the laboratory reporting level to compute statistic]

\begin{tabular}{|c|c|c|c|c|c|}
\hline Property or constituent and reporting unit & $\begin{array}{c}\text { Number of } \\
\text { samples }\end{array}$ & Maximum & Minimum & Mean & Median \\
\hline \multicolumn{6}{|c|}{$\begin{array}{l}12334550 \text { - Clark Fork at Turah Bridge, near Bonner } \\
\text { Period of record for water-quality data: March 1985-September } 2005\end{array}$} \\
\hline Streamflow, instantaneous $\left(\mathrm{ft}^{3} / \mathrm{s}\right)$ & 204 & 9,560 & 296 & 1,860 & 1,110 \\
\hline $\mathrm{pH}$, onsite (standard units) & 150 & 8.8 & 7.4 & 8.2 & 8.3 \\
\hline Specific conductance, onsite $(\mu \mathrm{S} / \mathrm{cm})$ & 179 & 483 & 139 & 304 & 316 \\
\hline Temperature, water $\left({ }^{\circ} \mathrm{C}\right)$ & 203 & 22.0 & 0.0 & 9.4 & 9.5 \\
\hline Hardness, filtered $\left(\mathrm{mg} / \mathrm{L}\right.$ as $\left.\mathrm{CaCO}_{3}\right)$ & 140 & 210 & 54 & 132 & 135 \\
\hline Calcium, filtered $(\mathrm{mg} / \mathrm{L})$ & 140 & 59.0 & 14.9 & 37.3 & 38.2 \\
\hline Magnesium, filtered (mg/L) & 140 & 14.0 & 3.94 & 9.49 & 9.50 \\
\hline Arsenic, filtered $(\mu \mathrm{g} / \mathrm{L})$ & 149 & 17 & 2.7 & 6.0 & 5.2 \\
\hline Arsenic, unfiltered $(\mu \mathrm{g} / \mathrm{L})$ & 149 & 110 & 3 & 10 & 7 \\
\hline Cadmium, filtered $(\mu \mathrm{g} / \mathrm{L})$ & 149 & .11 & $<.04$ & ${ }^{2} .03$ & $<.1$ \\
\hline Cadmium, unfiltered $(\mu \mathrm{g} / \mathrm{L})$ & 149 & 4 & $<.1$ & ${ }^{2} .28$ & $<1$ \\
\hline Copper, filtered $(\mu \mathrm{g} / \mathrm{L})$ & 148 & 25 & E1.1 & 5.0 & 4.0 \\
\hline Copper, unfiltered $(\mu \mathrm{g} / \mathrm{L})$ & 147 & 500 & 3 & 37.9 & 17.6 \\
\hline Iron, filtered $(\mu \mathrm{g} / \mathrm{L})$ & 149 & 190 & $<3$ & 224 & 13 \\
\hline Iron, unfiltered $(\mu \mathrm{g} / \mathrm{L})$ & 149 & 19,000 & 30 & 1,120 & 390 \\
\hline Lead, filtered $(\mu \mathrm{g} / \mathrm{L})$ & 145 & 7 & $<.08$ & ${ }^{2} .34$ & $<1$ \\
\hline Lead, unfiltered $(\mu \mathrm{g} / \mathrm{L})$ & 145 & 100 & $<1$ & 27.93 & 3.00 \\
\hline Manganese, filtered $(\mu \mathrm{g} / \mathrm{L})$ & 149 & 37.4 & 1.0 & 8.2 & 7.0 \\
\hline Manganese, unfiltered $(\mu \mathrm{g} / \mathrm{L})$ & 149 & 2,000 & 10 & 134 & 70 \\
\hline Zinc, filtered $(\mu \mathrm{g} / \mathrm{L})$ & 148 & 39 & $<3$ & ${ }^{2} 6.5$ & 4.2 \\
\hline Zinc, unfiltered $(\mu \mathrm{g} / \mathrm{L})$ & 149 & 1,100 & $<10$ & ${ }^{2} 66$ & 30 \\
\hline Sediment, suspended (percent finer than $0.062 \mathrm{~mm}$ ) & 193 & 98 & 27 & 73 & 75 \\
\hline Sediment, suspended concentration $(\mathrm{mg} / \mathrm{L})$ & 204 & 1,370 & 2 & 60 & 18 \\
\hline Sediment, suspended discharge (ton/d) & 204 & 34,700 & 3.5 & 664 & 60 \\
\hline
\end{tabular}


Table 20. Statistical summary of water-quality data for the upper Clark Fork basin, Montana, March 1985 through September 2005. -Continued

[Abbreviations: $\mathrm{ft}^{3} / \mathrm{s}$, cubic feet per second; ${ }^{\circ} \mathrm{C}$, degrees Celsius; E, estimated; $\mu \mathrm{g} / \mathrm{L}$, micrograms per liter; $\mu \mathrm{S} / \mathrm{cm}, \mathrm{microsiemens} \mathrm{per} \mathrm{centimeter} \mathrm{at} 25^{\circ} \mathrm{C}$; $\mathrm{mg} / \mathrm{L}$, milligrams per liter; mm, millimeter; ton/d, tons per day. Symbols: <, less than laboratory reporting level ${ }^{1}$; --, indicates insufficient data greater than the laboratory reporting level to compute statistic]

\begin{tabular}{|c|c|c|c|c|c|}
\hline Property or constituent and reporting unit & $\begin{array}{l}\text { Number of } \\
\text { samples }\end{array}$ & Maximum & Minimum & Mean & Median \\
\hline \multicolumn{6}{|c|}{$\begin{array}{l}12340000 \text {-Blackfoot River near Bonner } \\
\text { Period of record for water-quality data: March 1985-September } 2005\end{array}$} \\
\hline Streamflow, instantaneous $\left(\mathrm{ft}^{3} / \mathrm{s}\right)$ & 146 & 13,400 & 344 & 2,650 & 1,310 \\
\hline pH, onsite (standard units) & 106 & 8.7 & 7.5 & 8.3 & 8.3 \\
\hline Hardness, filtered $\left(\mathrm{mg} / \mathrm{L}\right.$ as $\left.\mathrm{CaCO}_{3}\right)$ & 99 & 140 & 55 & 102 & 97 \\
\hline Calcium, filtered (mg/L) & 99 & 37 & 14 & 26.3 & 24.8 \\
\hline Magnesium, filtered (mg/L) & 99 & 13 & 4.9 & 9.08 & 8.63 \\
\hline Arsenic, filtered $(\mu \mathrm{g} / \mathrm{L})$ & 106 & 2 & $<1$ & ${ }^{2} 1.0$ & 1.0 \\
\hline Copper, filtered $(\mu \mathrm{g} / \mathrm{L})$ & 104 & 7 & $<1$ & ${ }^{2} 1.5$ & .9 \\
\hline Copper, unfiltered ( $\mu \mathrm{g} / \mathrm{L})$ & 103 & 34 & $<1$ & ${ }^{2} 5.6$ & 2.6 \\
\hline Iron, filtered $(\mu \mathrm{g} / \mathrm{L})$ & 106 & 100 & $<3$ & ${ }^{2} 18$ & 10 \\
\hline Iron, unfiltered $(\mu \mathrm{g} / \mathrm{L})$ & 106 & 3,600 & 10 & 463 & 205 \\
\hline Lead, filtered $(\mu \mathrm{g} / \mathrm{L})$ & 102 & 8 & $<.08$ & ${ }^{2} .46$ & $<1$ \\
\hline Lead, unfiltered ( $\mu \mathrm{g} / \mathrm{L})$ & 102 & 25 & $<.06$ & ${ }^{2} 2.86$ & $<5$ \\
\hline Manganese, filtered $(\mu \mathrm{g} / \mathrm{L})$ & 106 & 11 & $<1$ & ${ }^{2} 2.4$ & 2.0 \\
\hline Manganese, unfiltered ( $\mu \mathrm{g} / \mathrm{L})$ & 106 & 180 & $<10$ & 231 & 20 \\
\hline
\end{tabular}


Table 20. Statistical summary of water-quality data for the upper Clark Fork basin, Montana, March 1985 through September 2005. -Continued

[Abbreviations: $\mathrm{ft}^{3} / \mathrm{s}$, cubic feet per second; ${ }^{\circ} \mathrm{C}$, degrees Celsius; E, estimated; $\mu \mathrm{g} / \mathrm{L}$, micrograms per liter; $\mu \mathrm{S} / \mathrm{cm}, \mathrm{microsiemens}$ per centimeter at $25^{\circ} \mathrm{C}$; $\mathrm{mg} / \mathrm{L}$, milligrams per liter; mm, millimeter; ton/d, tons per day. Symbols: <, less than laboratory reporting level ${ }^{1}$; --, indicates insufficient data greater than the laboratory reporting level to compute statistic]

\begin{tabular}{|c|c|c|c|c|c|}
\hline Property or constituent and reporting unit & $\begin{array}{l}\text { Number of } \\
\text { samples }\end{array}$ & Maximum & Minimum & Mean & Median \\
\hline \multicolumn{6}{|c|}{$\begin{array}{l}12340500 \text { —Clark Fork above Missoula } \\
\text { Period of record for water-quality data: July 1986-September } 2005\end{array}$} \\
\hline Streamflow, instantaneous $\left(\mathrm{ft}^{3} / \mathrm{s}\right)$ & 170 & 21,600 & 720 & 4,400 & 2,380 \\
\hline $\mathrm{pH}$, onsite (standard units) & 127 & 8.7 & 7.9 & 8.3 & 8.3 \\
\hline Specific conductance, onsite $(\mu \mathrm{S} / \mathrm{cm})$ & 147 & 399 & 142 & 254 & 261 \\
\hline Temperature, water $\left({ }^{\circ} \mathrm{C}\right)$ & 167 & 21.0 & 0.0 & 9.4 & 9.0 \\
\hline Hardness, filtered $\left(\mathrm{mg} / \mathrm{L}\right.$ as $\left.\mathrm{CaCO}_{3}\right)$ & 127 & 170 & 61 & 117 & 120 \\
\hline Calcium, filtered (mg/L) & 127 & 46 & 14 & 31.5 & 32.0 \\
\hline Magnesium, filtered (mg/L) & 127 & 13.0 & 5.28 & 9.26 & 9.20 \\
\hline Arsenic, filtered $(\mu \mathrm{g} / \mathrm{L})$ & 127 & 9 & 1 & 3.3 & 3.0 \\
\hline Arsenic, unfiltered $(\mu \mathrm{g} / \mathrm{L})$ & 127 & 69 & 1 & 5 & 4 \\
\hline Cadmium, filtered $(\mu \mathrm{g} / \mathrm{L})$ & 127 & .2 & $<.04$ & ${ }^{2} .03$ & $<.1$ \\
\hline Cadmium, unfiltered $(\mu \mathrm{g} / \mathrm{L})$ & 127 & 5 & $<.1$ & ${ }^{2} .14$ & $<1$ \\
\hline Copper, filtered $(\mu \mathrm{g} / \mathrm{L})$ & 126 & 12.6 & .7 & 2.8 & 2.0 \\
\hline Copper, unfiltered $(\mu \mathrm{g} / \mathrm{L})$ & 125 & 400 & 2 & 16.4 & 8.0 \\
\hline Iron, filtered $(\mu \mathrm{g} / \mathrm{L})$ & 127 & 200 & $<3$ & 222 & 15 \\
\hline Iron, unfiltered $(\mu \mathrm{g} / \mathrm{L})$ & 127 & 13,000 & 40 & 598 & 230 \\
\hline Lead, filtered $(\mu \mathrm{g} / \mathrm{L})$ & 122 & 1.2 & $<.08$ & ${ }^{2} .17$ & $<1$ \\
\hline Lead, unfiltered $(\mu \mathrm{g} / \mathrm{L})$ & 122 & 78 & $<1$ & 23.12 & 1.17 \\
\hline Manganese, filtered $(\mu \mathrm{g} / \mathrm{L})$ & 127 & 230 & 6.2 & 17.6 & 14.2 \\
\hline Manganese, unfiltered $(\mu \mathrm{g} / \mathrm{L})$ & 127 & 1,100 & 10 & 65 & 40 \\
\hline Zinc, filtered $(\mu \mathrm{g} / \mathrm{L})$ & 127 & 16 & $<1$ & 23.8 & 2.1 \\
\hline Zinc, unfiltered ( $\mu \mathrm{g} / \mathrm{L})$ & 127 & 1,100 & $<10$ & ${ }^{2} 31$ & 12 \\
\hline Sediment, suspended (percent finer than $0.062 \mathrm{~mm}$ ) & 165 & 99 & 44 & 87 & 90 \\
\hline Sediment, suspended concentration (mg/L) & 170 & 824 & 2 & 37 & 12 \\
\hline Sediment, suspended discharge (ton/d) & 170 & 21,900 & 5.8 & 945 & 68 \\
\hline
\end{tabular}

${ }^{1}$ Differing less-than $(<)$ values for an individual constituent are the result of changes in the laboratory reporting level during the period of record.

${ }^{2}$ Value for the mean is estimated by using a log-probability regression to predict the values of data less than the laboratory reporting level (Helsel and Cohn, 1988). 
Table 21. Statistical summary of fine-grained bed-sediment data for the upper Clark Fork basin, Montana, August 1986 through August 2005.

[For water year 2005, bulk bed (fine-grained plus coarse-grained) sediment was not collected as in previous years. Bed-sediment data consist of only the fine-grained fraction (material less than 0.064 millimeter in diameter). Reported concentrations are in micrograms per gram dry weight. Number of samples represents the number of years that the constituent was analyzed, with each year represented by a single mean concentration of composite samples. Values for a single sample are arbitrarily listed in the "Mean" column. Values are reported using U.S. Geological Survey rounding standards. Symbols: $<$, less than the minimum reporting level; --, indicates insufficient data to compute statistic]

\begin{tabular}{|c|c|c|c|c|c|}
\hline Constituent & Number of samples & Maximum & Minimum & Mean & Median \\
\hline \multicolumn{6}{|c|}{$\begin{array}{l}12323600 \text {-Silver Bow Creek at Opportunity } \\
\text { Period of record for bed-sediment data: 1992-2005 }\end{array}$} \\
\hline Arsenic & 3 & 186 & 163 & 171 & 165 \\
\hline Cadmium & 14 & 43.9 & 23.7 & 34.0 & 34.6 \\
\hline Chromium & 12 & 32.4 & 16.8 & 24.8 & 24.5 \\
\hline Copper & 14 & 9,020 & 3,390 & 4,940 & 4,620 \\
\hline Iron & 14 & 41,200 & 28,200 & 35,600 & 36,000 \\
\hline Lead & 14 & 1,030 & 381 & 726 & 792 \\
\hline Manganese & 14 & 9,220 & 1,680 & 3,710 & 2,970 \\
\hline Nickel & 13 & 21.4 & 12.4 & 15.4 & 15.3 \\
\hline Silver & 12 & 20.0 & 8.3 & 15.5 & 15.8 \\
\hline Zinc & 14 & 13,400 & 5,620 & 8,460 & 7,820 \\
\hline
\end{tabular}

\begin{tabular}{|c|c|c|c|c|c|}
\hline Zinc & 14 & 13,400 & 5,620 & 8,460 & 7,820 \\
\hline \multicolumn{6}{|c|}{$\begin{array}{l}\text { 12323750-Silver Bow Creek at Warm Springs } \\
\text { Period of record for bed-sediment data: 1992-2005 }\end{array}$} \\
\hline Arsenic & 3 & 177 & 103 & 141 & 141 \\
\hline Cadmium & 14 & 12.2 & 4.2 & 7.7 & 7.1 \\
\hline Chromium & 12 & 34.1 & $<15.7$ & 19.4 & 18.5 \\
\hline Copper & 14 & 769 & 169 & 355 & 279 \\
\hline Iron & 14 & 31,700 & 15,400 & 22,200 & 20,800 \\
\hline Lead & 14 & 100 & 49 & 71 & 71 \\
\hline Manganese & 14 & 17,700 & 1,470 & 8,490 & 8,370 \\
\hline Nickel & 13 & 19.1 & 9.2 & 14.8 & 14.6 \\
\hline Silver & 12 & 4.4 & .3 & ${ }^{1} 1.9$ & ${ }^{1} 1.8$ \\
\hline Zinc & 14 & 2,220 & 620 & 1,040 & 828 \\
\hline
\end{tabular}

12323770 - Warm Springs Creek at Warm Springs

Period of record for bed-sediment data: 1995, 1997, 1999, 2002, 2005

\begin{tabular}{|c|c|c|c|c|c|}
\hline Arsenic & 1 & -- & -- & 66 & -- \\
\hline Cadmium & 5 & 5.8 & 1.3 & 3.6 & 3.9 \\
\hline Chromium & 5 & 33.4 & 27.5 & 30.4 & 30.8 \\
\hline Copper & 5 & 991 & 779 & 878 & 881 \\
\hline Iron & 5 & 22,400 & 16,800 & 20,700 & 21,900 \\
\hline Lead & 5 & 86 & 67 & 81 & 85 \\
\hline Manganese & 5 & 12,100 & 2,020 & 7,980 & 8,790 \\
\hline Nickel & 5 & 21.9 & 17.6 & 19.2 & 19.2 \\
\hline Silver & 4 & 5.1 & 3.1 & 3.8 & 3.5 \\
\hline Zinc & 5 & 421 & 372 & 395 & 396 \\
\hline
\end{tabular}


Table 21. Statistical summary of fine-grained bed-sediment data for the upper Clark Fork basin, Montana, August 1986 through August 2005.-Continued

[For water year 2005, bulk bed (fine-grained plus coarse-grained) sediment was not collected as in previous years. Bed-sediment data consist of only the fine-grained fraction (material less than 0.064 millimeter in diameter). Reported concentrations are in micrograms per gram dry weight. Number of samples represents the number of years that the constituent was analyzed, with each year represented by a single mean concentration of composite samples. Values for a single sample are arbitrarily listed in the "Mean" column. Values are reported using U.S. Geological Survey rounding standards. Symbols: <, less than the minimum reporting level; --, indicates insufficient data to compute statistic]

\begin{tabular}{|c|c|c|c|c|c|}
\hline Constituent & Number of samples & Maximum & Minimum & Mean & Median \\
\hline \multicolumn{6}{|c|}{$\begin{array}{l}12323800 \text {-Clark Fork near Galen } \\
\text { Period of record for bed-sediment data: 1987, 1991-2005 }\end{array}$} \\
\hline Arsenic & 3 & 119 & 107 & 112 & 111 \\
\hline Cadmium & 16 & 20.1 & 4.0 & 9.5 & 8.5 \\
\hline Chromium & 12 & 33.9 & 19.1 & 26.2 & 26.1 \\
\hline Copper & 16 & 2,300 & 838 & 1,230 & 1,130 \\
\hline Iron & 16 & 39,800 & 22,600 & 27,800 & 26,900 \\
\hline Lead & 16 & 235 & 92 & 139 & 134 \\
\hline Manganese & 16 & 17,300 & 2,780 & 9,560 & 10,200 \\
\hline Nickel & 13 & 23.2 & 13.9 & 18.2 & 18.2 \\
\hline Silver & 14 & 7.3 & $<3.2$ & ${ }^{1} 4.4$ & ${ }^{1} 4.5$ \\
\hline Zinc & 16 & 3,560 & 999 & 1,610 & 1,200 \\
\hline
\end{tabular}

461415112450801-Clark Fork below Lost Creek, near Galen

Period of record for bed-sediment data: 1996-2005

\begin{tabular}{|c|c|c|c|c|c|}
\hline Arsenic & 3 & 204 & 92 & 134 & 107 \\
\hline Cadmium & 10 & 10.5 & 5.8 & 7.8 & 7.7 \\
\hline Chromium & 9 & 34.5 & 20.5 & 27.9 & 27.5 \\
\hline Copper & 10 & 2,050 & 1,150 & 1,510 & 1,430 \\
\hline Iron & 10 & 32,800 & 24,400 & 29,600 & 30,500 \\
\hline Lead & 10 & 218 & 127 & 176 & 178 \\
\hline Manganese & 10 & 9,670 & 3,540 & 5,650 & 5,660 \\
\hline Nickel & 10 & 19.9 & 11.7 & 16.2 & 164.0 \\
\hline Silver & 8 & 7.8 & 4.2 & 6.5 & 6.7 \\
\hline Zinc & 10 & 1,680 & 1,120 & 1,380 & 1,380 \\
\hline
\end{tabular}

461559112443301 — Clark Fork at County Bridge, near Racetrack

Period of record for bed-sediment data: 1996-2005

\begin{tabular}{lrcccrr}
\hline Arsenic & 3 & 101 & 56 & 81 & 86.00 \\
Cadmium & 10 & 8.7 & 5.0 & 7.0 & 2.0 \\
Chromium & 9 & 33.3 & 19.0 & 25.0 & 1,170 \\
Copper & 10 & 1,610 & 933 & 1,190 & 26,400 \\
Iron & 10 & 31,700 & 21,200 & 26,300 & 138 \\
Lead & 10 & 186 & 103 & 3,670 & 3,560 \\
Manganese & 10 & 6,310 & 2,100 & 14.0 & 14.1 \\
Nickel & 10 & 18.4 & 10.3 & 15.0 & 15.4 \\
Silver & 8 & 6.1 & $<3.3$ & 1,210 & 1,150
\end{tabular}


Table 21. Statistical summary of fine-grained bed-sediment data for the upper Clark Fork basin, Montana, August 1986 through August 2005.-Continued

[For water year 2005, bulk bed (fine-grained plus coarse-grained) sediment was not collected as in previous years. Bed-sediment data consist of only the fine-grained fraction (material less than 0.064 millimeter in diameter). Reported concentrations are in micrograms per gram dry weight. Number of samples represents the number of years that the constituent was analyzed, with each year represented by a single mean concentration of composite samples. Values for a single sample are arbitrarily listed in the "Mean" column. Values are reported using U.S. Geological Survey rounding standards. Symbols: <, less than the minimum reporting level; --, indicates insufficient data to compute statistic]

\begin{tabular}{|c|c|c|c|c|c|}
\hline Constituent & Number of samples & Maximum & Minimum & Mean & Median \\
\hline \multicolumn{6}{|c|}{$\begin{array}{l}\text { 461903112440701 —Clark Fork at Dempsey Creek Diversion, near Racetrack } \\
\text { Period of record for bed-sediment data: 1996-2005 }\end{array}$} \\
\hline Arsenic & 3 & 80 & 58 & 70 & 71 \\
\hline Cadmium & 10 & 10.3 & 4.3 & 6.8 & 6.4 \\
\hline Chromium & 9 & 34.1 & 16.0 & 24.7 & 25.6 \\
\hline Copper & 10 & 1,550 & 721 & 1,020 & 996 \\
\hline Iron & 10 & 33,700 & 20,600 & 26,100 & 25,200 \\
\hline Lead & 10 & 155 & 92 & 129 & 126 \\
\hline Manganese & 10 & 8,370 & 1,810 & 4,060 & 3,290 \\
\hline Nickel & 10 & 16.9 & 8.7 & 13.0 & 12.7 \\
\hline Silver & 8 & 6.2 & 2.7 & 4.9 & 5.0 \\
\hline Zinc & 10 & 1,570 & 900 & 1,130 & 1,080 \\
\hline
\end{tabular}

12324200 - Clark Fork at Deer Lodge

Period of record for bed-sediment data: 1986-1987, 1990-2005

\begin{tabular}{lccccr}
\hline Arsenic & 3 & 77 & 49 & 66 & 72 \\
Cadmium & 18 & 10.0 & 3.8 & 6.6 & 3.2 \\
Chromium & 12 & 43.9 & 19.5 & 30.3 & 1,060 \\
Copper & 18 & 4,180 & 683 & 1,320 & 26,400 \\
Iron & 18 & 35,300 & 21,100 & 27,400 & 149 \\
Lead & 18 & 242 & 103 & 150 & 2,420 \\
Manganese & 18 & 6,020 & 1,110 & 130 & 15.0 \\
Nickel & 13 & 21.1 & 11.5 & 4.7 & 4.5 \\
Silver & 16 & 7.9 & 2.4 & 1,240 & 1,260 \\
Zinc & 18 & 1,730 & 846 & 4.4 & \\
\hline
\end{tabular}

12324590 — Little Blackfoot River near Garrison

Period of record for bed-sediment data: 1986-1987, 1994, 1998, 2001, 2004

\begin{tabular}{llccrr}
\hline Arsenic & 1 & -- & -- & 12 & -- \\
Cadmium & 6 & 2.3 & 0.2 & 1.1 & 0.9 \\
Chromium & 4 & 54.4 & 22.1 & 40.6 & 43.0 \\
Copper & 6 & 85 & 33 & 52 & 40 \\
Iron & 6 & 30,700 & 16,100 & 24,300 & 24,400 \\
Lead & 6 & 53 & 36 & 40 & 38 \\
Manganese & 6 & 2,700 & 905 & 1,370 & 1,080 \\
Nickel & 4 & 21.9 & 13.6 & 17.6 & 17.4 \\
Silver & 5 & .9 & 161 & 1.6 & $1<1.5$ \\
Zinc & 6 & 204 & & 176 & 173
\end{tabular}


Table 21. Statistical summary of fine-grained bed-sediment data for the upper Clark Fork basin, Montana, August 1986 through August 2005.-Continued

[For water year 2005, bulk bed (fine-grained plus coarse-grained) sediment was not collected as in previous years. Bed-sediment data consist of only the fine-grained fraction (material less than 0.064 millimeter in diameter). Reported concentrations are in micrograms per gram dry weight. Number of samples represents the number of years that the constituent was analyzed, with each year represented by a single mean concentration of composite samples. Values for a single sample are arbitrarily listed in the "Mean" column. Values are reported using U.S. Geological Survey rounding standards. Symbols: <, less than the minimum reporting level; --, indicates insufficient data to compute statistic]

\begin{tabular}{|c|c|c|c|c|c|}
\hline Constituent & Number of samples & Maximum & Minimum & Mean & Median \\
\hline \multicolumn{6}{|c|}{$\begin{array}{l}12324680 \text {-Clark Fork at Goldcreek } \\
\text { Period of record for bed-sediment data: } 1992-2005\end{array}$} \\
\hline Arsenic & 3 & 39 & 24 & 32 & 32 \\
\hline Cadmium & 14 & 8.1 & 3.1 & 5.1 & 5.4 \\
\hline Chromium & 12 & 48.9 & 22.1 & 32.1 & 31.8 \\
\hline Copper & 14 & 1,080 & 393 & 693 & 738 \\
\hline Iron & 14 & 30,600 & 19,500 & 23,800 & 24,000 \\
\hline Lead & 14 & 152 & 61 & 98 & 99 \\
\hline Manganese & 14 & 2,610 & 1,160 & 1,890 & 1,810 \\
\hline Nickel & 13 & 18.6 & 10.9 & 14.7 & 15.0 \\
\hline Silver & 12 & 4.8 & 2.3 & 3.3 & 3.2 \\
\hline Zinc & 14 & 1,320 & 590 & 978 & 1,080 \\
\hline \multicolumn{6}{|c|}{$\begin{array}{l}12331800 \text { —Clark Fork near Drummond } \\
\text { Period of record for bed-sediment data: 1986-1987, 1991-2005 }\end{array}$} \\
\hline Arsenic & 3 & 34 & 31 & 32 & 33 \\
\hline Cadmium & 17 & 7.7 & 2.6 & 4.7 & 4.8 \\
\hline Chromium & 12 & 35.4 & 17.0 & 28.6 & 30.8 \\
\hline Copper & 17 & 747 & 321 & 495 & 491 \\
\hline Iron & 17 & 27,000 & 16,500 & 21,500 & 20,400 \\
\hline Lead & 17 & 135 & 64 & 91 & 93 \\
\hline Manganese & 17 & 3,090 & 1,150 & 1,900 & 1,880 \\
\hline Nickel & 13 & 16.8 & 10.4 & 13.7 & 14.0 \\
\hline Silver & 15 & 4.7 & $<3.2$ & 13.0 & ${ }^{1} 2.9$ \\
\hline Zinc & 17 & 1,230 & 742 & 991 & 1,000 \\
\hline
\end{tabular}

12334510-Rock Creek near Clinton

Period of record for bed-sediment data: 1986-1987, 1989, 1991-1999, 2001-2005

\begin{tabular}{lccccc}
\hline Arsenic & 3 & 6 & $<6$ & ${ }^{15}$ & ${ }^{1} 5$ \\
Cadmium & 17 & 3.7 & $<.1$ & 21.8 & 20.5 \\
Chromium & 11 & 27.9 & 16.1 & 11 & 12 \\
Copper & 17 & 16 & 3 & 17,300 & 17,200 \\
Iron & 17 & 21,400 & 13,100 & 9 & 10 \\
Lead & 17 & 16 & 126 & 373 & 361 \\
Manganese & 17 & 724 & 8.7 & 11.5 & 11.4 \\
Nickel & 12 & 14.8 & $<.3$ & 1.5 & $1<.6$ \\
Silver & 14 & 1.9 & 23 & 42 & 45
\end{tabular}


Table 21. Statistical summary of fine-grained bed-sediment data for the upper Clark Fork basin, Montana, August 1986 through August 2005.-Continued

[For water year 2005, bulk bed (fine-grained plus coarse-grained) sediment was not collected as in previous years. Bed-sediment data consist of only the fine-grained fraction (material less than 0.064 millimeter in diameter). Reported concentrations are in micrograms per gram dry weight. Number of samples represents the number of years that the constituent was analyzed, with each year represented by a single mean concentration of composite samples. Values for a single sample are arbitrarily listed in the "Mean" column. Values are reported using U.S. Geological Survey rounding standards. Symbols: <, less than the minimum reporting level; --, indicates insufficient data to compute statistic]

\begin{tabular}{|c|c|c|c|c|c|}
\hline Constituent & Number of samples & Maximum & Minimum & Mean & Median \\
\hline \multicolumn{6}{|c|}{$\begin{array}{l}12334550 \text {-Clark Fork at Turah Bridge, near Bonner } \\
\text { Period of record for bed-sediment data: 1986, 1991-2005 }\end{array}$} \\
\hline Arsenic & 3 & 30 & 19 & 24 & 22 \\
\hline Cadmium & 16 & 7.3 & 2.3 & 4.0 & 3.9 \\
\hline Chromium & 12 & 34.7 & 15.3 & 24.6 & 27.2 \\
\hline Copper & 16 & 635 & 211 & 363 & 322 \\
\hline Iron & 16 & 24,400 & 12,600 & 18,700 & 17,300 \\
\hline Lead & 16 & 115 & 47 & 71 & 65 \\
\hline Manganese & 16 & 2,270 & 671 & 1,230 & 1,200 \\
\hline Nickel & 13 & 19.1 & 8.7 & 12.9 & 11.6 \\
\hline Silver & 14 & 3.9 & $<1.9$ & ${ }^{1} 2.1$ & ${ }^{1} 1.9$ \\
\hline Zinc & 16 & 1,160 & 586 & 848 & 816 \\
\hline
\end{tabular}

12340000 -Blackfoot River near Bonner

Period of record for bed-sediment data: 1986-1987, 1991, 1993-1996, 1998-2001, 2003

\begin{tabular}{|c|c|c|c|c|c|}
\hline Arsenic & 1 & -- & -- & 2 & -- \\
\hline Cadmium & 12 & 2.0 & $<0.2$ & ${ }^{1} .7$ & ${ }^{1}<1.0$ \\
\hline Chromium & 8 & 25.8 & 15.1 & 20.5 & 21.8 \\
\hline Copper & 12 & 27 & 11 & 20 & 21 \\
\hline Iron & 12 & 20,200 & 12,400 & 16,800 & 16,800 \\
\hline Lead & 12 & 20 & $<13$ & ${ }^{1} 13$ & ${ }^{1} 12$ \\
\hline Manganese & 12 & 683 & 298 & 531 & 538 \\
\hline Nickel & 9 & 14.3 & 9.4 & 11.9 & 12.5 \\
\hline Silver & 12 & $<1.9$ & $<.3$ & ${ }^{1} .5$ & ${ }^{1}<.6$ \\
\hline Zinc & 12 & 73 & 35 & 58 & 61 \\
\hline \multicolumn{6}{|c|}{$\begin{array}{l}12340500 \text { - Clark Fork above Missoula } \\
\text { Period of record for bed-sediment data: 1997-2005 }\end{array}$} \\
\hline Arsenic & 3 & 29 & 17 & 23 & 23 \\
\hline Cadmium & 9 & 5.8 & 1.5 & 3.4 & 3.4 \\
\hline Chromium & 8 & 30.6 & 19.0 & 25.0 & 24.6 \\
\hline Copper & 9 & 543 & 166 & 328 & 282 \\
\hline Iron & 9 & 24,300 & 18,100 & 20,300 & 20,400 \\
\hline Lead & 9 & 78 & 37 & 55 & 54 \\
\hline Manganese & 9 & 1,420 & 477 & 977 & 970 \\
\hline Nickel & 9 & 15.8 & 10.9 & 13.2 & 13.0 \\
\hline Silver & 7 & 2.9 & .8 & 12.0 & ${ }^{12} .1$ \\
\hline Zinc & 9 & 1,090 & 438 & 709 & 696 \\
\hline
\end{tabular}


Table 21. Statistical summary of fine-grained bed-sediment data for the upper Clark Fork basin, Montana, August 1986 through August 2005.-Continued

[For water year 2005, bulk bed (fine-grained plus coarse-grained) sediment was not collected as in previous years. Bed-sediment data consist of only the fine-grained fraction (material less than 0.064 millimeter in diameter). Reported concentrations are in micrograms per gram dry weight. Number of samples represents the number of years that the constituent was analyzed, with each year represented by a single mean concentration of composite samples. Values for a single sample are arbitrarily listed in the "Mean" column. Values are reported using U.S. Geological Survey rounding standards. Symbols: <, less than the minimum reporting level; --, indicates insufficient data to compute statistic]

\begin{tabular}{|c|c|c|c|c|c|}
\hline Constituent & Number of samples & Maximum & Minimum & Mean & Median \\
\hline \multicolumn{6}{|c|}{$\begin{array}{l}12353000 \text {-Clark Fork below Missoula² } \\
\text { Period of record for bed-sediment data: 1986, 1990-2004 }\end{array}$} \\
\hline Arsenic & 2 & 14 & 6 & -- & -- \\
\hline Cadmium & 16 & 6.0 & 1.1 & 2.3 & 1.9 \\
\hline Chromium & 11 & 27.6 & 12.3 & 21.4 & 21.5 \\
\hline Copper & 16 & 293 & 69 & 150 & 140 \\
\hline Iron & 16 & 21,100 & 13,100 & 18,100 & 18,600 \\
\hline Lead & 16 & 58 & 12 & 36 & 36 \\
\hline Manganese & 16 & 2,530 & 446 & 1,360 & 1,270 \\
\hline Nickel & 12 & 14.1 & 8.4 & 12.0 & 12.5 \\
\hline Silver & 15 & 3.0 & .4 & ${ }^{1} 1.3$ & ${ }^{1} 1.2$ \\
\hline Zinc & 16 & 675 & 239 & 396 & 395 \\
\hline
\end{tabular}

${ }^{1}$ Value was determined by substituting one-half of the minimum reporting level for censored $(<)$ values when both uncensored and censored values were used to determine the mean and (or) median. When all data were less than the minimum reporting level, the median was determined by ranking the censored values by order of magnitude.

${ }^{2}$ Samples were collected about 30 miles downstream from streamflow-gaging station to conform to previous sampling location. 
Table 22. Statistical summary of biological data for the upper Clark Fork basin, Montana, August 1986 through August 2005.

[Concentrations are in micrograms per gram dry weight. Number of composite samples represents the total of all individual composite samples collected for every year that the constituent was analyzed. Values for a single sample are arbitraily listed in the "Mean" column. Because Hydropsyche insects were not sorted to the species level during 1986-89, Hydropsyche species statistics for stations sampled during those years are based on the results of all Hydropsyche species combined. At some sites, statistics of Hydropsyche morosa group are based on the combined results of two or more species. Insects collected during 1986-98 were depurated prior to analysis; depuration was discontinued in 1999. Arsenic data were not collected at some sites. Values are reported using U.S. Geological Survey rounding standards. Abbreviation: spp., one or more similar species. Symbols: <, less than minimum reporting level; --, indicates either too few samples (less than three) or insufficient data to compute statistic, or element not analyzed]

\begin{tabular}{|c|c|c|c|c|c|}
\hline Constituent & $\begin{array}{c}\text { Number of } \\
\text { composite samples }\end{array}$ & Maximum & Minimum & Mean & Median \\
\hline \multicolumn{6}{|c|}{$\begin{array}{c}12323600 \text { —Silver Bow Creek at Opportunity } \\
\text { Period of record for biological data: 1992, 1994-95, 1997-2005 }\end{array}$} \\
\hline \multicolumn{6}{|c|}{ Brachycentrus spp. } \\
\hline Arsenic & 0 & -- & -- & -- & -- \\
\hline Cadmium & 5 & 12.5 & 5.8 & 10.1 & 11.6 \\
\hline Chromium & 5 & 5.9 & .7 & 2.1 & .9 \\
\hline Copper & 5 & 846 & 235 & 587 & 592 \\
\hline Iron & 5 & 1,190 & 335 & 617 & 469 \\
\hline Lead & 5 & 21.5 & 7.4 & 13.7 & 13.8 \\
\hline Manganese & 5 & 817 & 231 & 515 & 503 \\
\hline Nickel & 5 & 2.1 & $<.1$ & ${ }^{1} 1.3$ & ${ }^{1} 1.6$ \\
\hline Zinc & 5 & 995 & 629 & 803 & 815 \\
\hline \multicolumn{6}{|c|}{ Hydropsyche cockerelli } \\
\hline Arsenic & 4 & 20.4 & 9.5 & 13.5 & 12.0 \\
\hline Cadmium & 10 & 9.7 & 4.1 & 5.8 & 5.1 \\
\hline Chromium & 10 & 8.0 & 1.0 & 2.9 & 1.9 \\
\hline Copper & 10 & 1,090 & 269 & 451 & 378 \\
\hline Iron & 10 & 2,660 & 689 & 1,390 & 1,120 \\
\hline Lead & 10 & 47.2 & 19.0 & 27.6 & 21.3 \\
\hline Manganese & 10 & 3,030 & 180 & 972 & 782 \\
\hline Nickel & 10 & 3.6 & .7 & 2.0 & 1.8 \\
\hline Zinc & 10 & 1,590 & 749 & 949 & 838 \\
\hline \multicolumn{6}{|c|}{ Hydropsyche spp. } \\
\hline Arsenic & 5 & 23.1 & 10.7 & 17.2 & 18.4 \\
\hline Cadmium & 10 & 10.9 & 4.6 & 7.0 & 5.9 \\
\hline Chromium & 10 & 4.7 & .6 & 2.1 & 1.5 \\
\hline Copper & 10 & 930 & 312 & 597 & 471 \\
\hline Iron & 10 & 2,550 & 1,050 & 1,860 & 1,990 \\
\hline Lead & 10 & 51.4 & 21.8 & 38.9 & 38.2 \\
\hline Manganese & 10 & 1,340 & 712 & 1,080 & 1,060 \\
\hline Nickel & 10 & 2.5 & .7 & 2.2 & 2.4 \\
\hline Zinc & 10 & 1,290 & 834 & 1,070 & 1,090 \\
\hline
\end{tabular}


Table 22. Statistical summary of biological data for the upper Clark Fork basin, Montana, August 1986 through August 2005. -Continued

[Concentrations are in micrograms per gram dry weight. Number of composite samples represents the total of all individual composite samples collected for every year that the constituent was analyzed. Values for a single sample are arbitraily listed in the "Mean" column. Because Hydropsyche insects were not sorted to the species level during 1986-89, Hydropsyche species statistics for stations sampled during those years are based on the results of all Hydropsyche species combined. At some sites, statistics of Hydropsyche morosa group are based on the combined results of two or more species. Insects collected during 1986-98 were depurated prior to analysis; depuration was discontinued in 1999. Arsenic data were not collected at some sites. Values are reported using U.S. Geological Survey rounding standards. Abbreviation: spp., one or more similar species. Symbols: <, less than minimum reporting level; --, indicates either too few samples (less than three) or insufficient data to compute statistic, or element not analyzed]

\begin{tabular}{|c|c|c|c|c|c|}
\hline Constituent & $\begin{array}{c}\text { Number of } \\
\text { composite samples }\end{array}$ & Maximum & Minimum & Mean & Median \\
\hline \multicolumn{6}{|c|}{$\begin{array}{l}12323600 \text {-Silver Bow Creek at Opportunity-Continued } \\
\text { Period of record for biological data: 1992, 1994-95, 1997-2005 }\end{array}$} \\
\hline \multicolumn{6}{|c|}{ Hydropsyche tana } \\
\hline Arsenic & 0 & -- & -- & -- & -- \\
\hline Cadmium & 6 & 9.2 & 4.8 & 6.8 & 6.9 \\
\hline Chromium & 6 & 11.5 & .9 & 4.5 & 1.8 \\
\hline Copper & 6 & 456 & 10.5 & 236 & 298 \\
\hline Iron & 6 & 1,520 & 875 & 1,100 & 1,050 \\
\hline Lead & 6 & 21.0 & 15.6 & 18.6 & 18.3 \\
\hline Manganese & 6 & 969 & 307 & 634 & 675 \\
\hline Nickel & 6 & 1.8 & .7 & 1.4 & 1.6 \\
\hline Zinc & 6 & 1,070 & 760 & 961 & 1,020 \\
\hline \multicolumn{6}{|c|}{$\begin{array}{l}12323750 \text {-Silver Bow Creek at Warm Springs } \\
\text { Period of record for biological data: 1992-2005 }\end{array}$} \\
\hline \multicolumn{6}{|c|}{ Hydropsyche cockerelli } \\
\hline Arsenic & 6 & 23.6 & 7.9 & 15.1 & 15.4 \\
\hline Cadmium & 32 & 2.1 & .2 & .7 & .5 \\
\hline Chromium & 32 & 4.3 & .4 & 1.0 & .8 \\
\hline Copper & 32 & 97.0 & 16.7 & 38.5 & 30.5 \\
\hline Iron & 32 & 1,590 & 351 & 770 & 761 \\
\hline Lead & 32 & 5.7 & .3 & 2.9 & 2.6 \\
\hline Manganese & 32 & 3,890 & 491 & 1,310 & 1,160 \\
\hline Nickel & 32 & 1.8 & .3 & .9 & .8 \\
\hline Zinc & 32 & 276 & 115 & 176 & 167 \\
\hline \multicolumn{6}{|c|}{ Hydropsyche occidentalils } \\
\hline Arsenic & 5 & 31.0 & 10.5 & 21.0 & 25.6 \\
\hline Cadmium & 20 & 1.6 & .2 & .6 & .4 \\
\hline Chromium & 20 & 6.8 & .3 & 1.7 & 1.0 \\
\hline Copper & 20 & 48.9 & 11.0 & 32.4 & 30.8 \\
\hline Iron & 20 & 2,960 & 372 & 1,190 & 971 \\
\hline Lead & 20 & 8.2 & $<1.7$ & ${ }^{1} 3.8$ & ${ }^{1} 3.5$ \\
\hline Manganese & 20 & 6,940 & 1,200 & 2,560 & 2,150 \\
\hline Nickel & 20 & 2.7 & .7 & 1.5 & 1.5 \\
\hline Zinc & 20 & 220 & 140 & 178 & 179 \\
\hline
\end{tabular}


Table 22. Statistical summary of biological data for the upper Clark Fork basin, Montana, August 1986 through August 2005. -Continued

[Concentrations are in micrograms per gram dry weight. Number of composite samples represents the total of all individual composite samples collected for every year that the constituent was analyzed. Values for a single sample are arbitraily listed in the "Mean" column. Because Hydropsyche insects were not sorted to the species level during 1986-89, Hydropsyche species statistics for stations sampled during those years are based on the results of all Hydropsyche species combined. At some sites, statistics of Hydropsyche morosa group are based on the combined results of two or more species. Insects collected during 1986-98 were depurated prior to analysis; depuration was discontinued in 1999. Arsenic data were not collected at some sites. Values are reported using U.S. Geological Survey rounding standards. Abbreviation: spp., one or more similar species. Symbols: <, less than minimum reporting level; --, indicates either too few samples (less than three) or insufficient data to compute statistic, or element not analyzed]

\begin{tabular}{|c|c|c|c|c|c|}
\hline Constituent & $\begin{array}{c}\text { Number of } \\
\text { composite samples }\end{array}$ & Maximum & Minimum & Mean & Median \\
\hline
\end{tabular}

12323750 - Silver Bow Creek at Warm Springs_Continued Period of record for biological data: 1992-2005

\begin{tabular}{|c|c|c|c|c|c|}
\hline \multicolumn{6}{|c|}{ Hydropsyche spp. } \\
\hline Arsenic & 0 & -- & -- & -- & -- \\
\hline Cadmium & 4 & 2.3 & 0.4 & 1.1 & 0.9 \\
\hline Chromium & 4 & 1.4 & .5 & 1.0 & 1.2 \\
\hline Copper & 4 & 47.6 & 34.9 & 40.9 & 40.6 \\
\hline Iron & 4 & 773 & 561 & 680 & 693 \\
\hline Lead & 4 & 5.1 & 1.9 & 3.9 & 4.7 \\
\hline Manganese & 4 & 1,100 & 443 & 725 & 678 \\
\hline Nickel & 4 & 1.9 & $<.4$ & ${ }^{1} .8$ & ${ }^{1} .5$ \\
\hline Zinc & 4 & 285 & 141 & 195 & 177 \\
\hline \multicolumn{6}{|c|}{$\begin{array}{l}12323770-\text { Warm Springs Creek at Warm Springs } \\
\text { Period of record for biological data: 1995, 1997, 1999, 2002, } 2005\end{array}$} \\
\hline \multicolumn{6}{|c|}{ Arctopsyche grandis } \\
\hline Arsenic & 2 & 9.8 & 9.5 & -- & -- \\
\hline Cadmium & 6 & 3.6 & 1.9 & 2.7 & 2.7 \\
\hline Chromium & 6 & 2.9 & .8 & 1.7 & 1.6 \\
\hline Copper & 6 & 133 & 78.3 & 106 & 100 \\
\hline Iron & 6 & 1,100 & 684 & 918 & 957 \\
\hline Lead & 6 & 5.6 & 3.0 & 14.7 & ${ }^{1} 5.2$ \\
\hline Manganese & 6 & 3,560 & 1,340 & 2,560 & 2,710 \\
\hline Nickel & 6 & 2.8 & 1.8 & 12.3 & ${ }^{1} 2.3$ \\
\hline Zinc & 6 & 222 & 181 & 196 & 196 \\
\hline \multicolumn{6}{|c|}{ Hydropsyche occidentalis } \\
\hline Arsenic & 2 & 13.6 & 12.7 & -- & -- \\
\hline Cadmium & 4 & 1.2 & .7 & 1.0 & 1.0 \\
\hline Chromium & 4 & 3.2 & .3 & 2.2 & 3.2 \\
\hline Copper & 4 & 183 & 125 & 156 & 158 \\
\hline Iron & 4 & 2,070 & 1,590 & 1,840 & 1,840 \\
\hline Lead & 4 & 8.2 & 6.7 & 7.4 & 7.4 \\
\hline Manganese & 4 & 3,190 & 2,400 & 2,790 & 2,770 \\
\hline Nickel & 4 & 3.3 & 2.0 & 2.6 & 2.6 \\
\hline Zinc & 4 & 172 & 148 & 160 & 160 \\
\hline
\end{tabular}


Table 22. Statistical summary of biological data for the upper Clark Fork basin, Montana, August 1986 through August 2005. -Continued

[Concentrations are in micrograms per gram dry weight. Number of composite samples represents the total of all individual composite samples collected for every year that the constituent was analyzed. Values for a single sample are arbitraily listed in the "Mean" column. Because Hydropsyche insects were not sorted to the species level during 1986-89, Hydropsyche species statistics for stations sampled during those years are based on the results of all Hydropsyche species combined. At some sites, statistics of Hydropsyche morosa group are based on the combined results of two or more species. Insects collected during 1986-98 were depurated prior to analysis; depuration was discontinued in 1999. Arsenic data were not collected at some sites. Values are reported using U.S. Geological Survey rounding standards. Abbreviation: spp., one or more similar species. Symbols: <, less than minimum reporting level; --, indicates either too few samples (less than three) or insufficient data to compute statistic, or element not analyzed]

\begin{tabular}{|c|c|c|c|c|c|}
\hline Constituent & $\begin{array}{c}\text { Number of } \\
\text { composite samples }\end{array}$ & Maximum & Minimum & Mean & Median \\
\hline \multicolumn{6}{|c|}{$\begin{array}{l}12323770-W a r m \text { Springs Creek at Warm Springs_Continued } \\
\text { Period of record for biological data: 1995, 1997, 1999, 2002, } 2005\end{array}$} \\
\hline \multicolumn{6}{|c|}{ Hydropsyche spp. } \\
\hline Arsenic & 0 & -- & -- & -- & -- \\
\hline Cadmium & 2 & 1.1 & 0.6 & -- & -- \\
\hline Chromium & 2 & 1.6 & 1.4 & -- & -- \\
\hline Copper & 2 & 95.9 & 94.8 & -- & -- \\
\hline Iron & 2 & 1,220 & 1,150 & -- & -- \\
\hline Lead & 2 & 5.9 & 5.2 & -- & -- \\
\hline Manganese & 2 & 3,390 & 956 & -- & -- \\
\hline Nickel & 2 & 2.0 & 1.8 & -- & -- \\
\hline Zinc & 2 & 129 & 125 & -- & -- \\
\hline \multicolumn{6}{|c|}{$\begin{array}{c}12323800 \text { - Clark Fork near Galen } \\
\text { Period of record for biological data: 1987, 1991-2005 }\end{array}$} \\
\hline \multicolumn{6}{|c|}{ Hydropsyche cockerelli } \\
\hline Arsenic & 3 & 15.8 & 13.2 & 14.2 & 13.6 \\
\hline Cadmium & 28 & 2.7 & .7 & 1.5 & 1.5 \\
\hline Chromium & 28 & 4.4 & .8 & 1.8 & 1.6 \\
\hline Copper & 28 & 181 & 48.7 & 100 & 98.1 \\
\hline Iron & 28 & 2,460 & 816 & 1,390 & 1,350 \\
\hline Lead & 28 & 11.7 & 1.2 & 7.6 & 7.6 \\
\hline Manganese & 28 & 3,620 & 1,070 & 2,280 & 2,320 \\
\hline Nickel & 28 & 3.1 & .9 & 1.6 & 1.5 \\
\hline Zinc & 28 & 299 & 136 & 210 & 208 \\
\hline \multicolumn{6}{|c|}{ Hydropsyche morosa group } \\
\hline Arsenic & 0 & -- & -- & -- & -- \\
\hline Cadmium & 5 & 3.2 & 2.4 & 2.5 & 2.4 \\
\hline Chromium & 5 & 4.6 & 1.8 & 2.6 & 2.2 \\
\hline Copper & 5 & 185 & 156 & 173 & 175 \\
\hline Iron & 5 & 1,890 & 1,360 & 1,510 & 1,430 \\
\hline Lead & 5 & 12.4 & 7.1 & 8.5 & 7.9 \\
\hline Manganese & 5 & 3,960 & 2,360 & 3,500 & 3,860 \\
\hline Nickel & 5 & 3.6 & 1.9 & 2.3 & 2.1 \\
\hline Zinc & 5 & 349 & 292 & 309 & 303 \\
\hline
\end{tabular}


Table 22. Statistical summary of biological data for the upper Clark Fork basin, Montana, August 1986 through August 2005. -Continued

[Concentrations are in micrograms per gram dry weight. Number of composite samples represents the total of all individual composite samples collected for every year that the constituent was analyzed. Values for a single sample are arbitraily listed in the "Mean" column. Because Hydropsyche insects were not sorted to the species level during 1986-89, Hydropsyche species statistics for stations sampled during those years are based on the results of all Hydropsyche species combined. At some sites, statistics of Hydropsyche morosa group are based on the combined results of two or more species. Insects collected during 1986-98 were depurated prior to analysis; depuration was discontinued in 1999. Arsenic data were not collected at some sites. Values are reported using U.S. Geological Survey rounding standards. Abbreviation: spp., one or more similar species. Symbols: <, less than minimum reporting level; --, indicates either too few samples (less than three) or insufficient data to compute statistic, or element not analyzed]

\begin{tabular}{|c|c|c|c|c|c|}
\hline Constituent & $\begin{array}{c}\text { Number of } \\
\text { composite samples }\end{array}$ & Maximum & Minimum & Mean & Median \\
\hline \multicolumn{6}{|c|}{$\begin{array}{l}12323800 \text {-Clark Fork near Galen—Continued } \\
\text { Period of record for biological data: 1987, 1991-2005 }\end{array}$} \\
\hline \multicolumn{6}{|c|}{ Hydropsyche occidentalis } \\
\hline Arsenic & 4 & 16.5 & 12.5 & 15.2 & 16.0 \\
\hline Cadmium & 36 & 1.7 & .6 & 1.1 & 1.1 \\
\hline Chromium & 36 & 6.6 & .4 & 2.0 & 1.5 \\
\hline Copper & 36 & 121 & 49.2 & 82.3 & 81.6 \\
\hline Iron & 36 & 1,920 & 642 & 1,270 & 1,220 \\
\hline Lead & 36 & 13.5 & 1.6 & 7.0 & 6.6 \\
\hline Manganese & 36 & 6,170 & 1,220 & 2,600 & 2,260 \\
\hline Nickel & 36 & 3.5 & .8 & 1.6 & 1.5 \\
\hline Zinc & 36 & 286 & 168 & 199 & 193 \\
\hline \multicolumn{6}{|c|}{ Hydropsyche tana } \\
\hline Arsenic & 0 & -- & -- & -- & -- \\
\hline Cadmium & 1 & -- & -- & 1.5 & -- \\
\hline Chromium & 1 & -- & -- & 1.4 & -- \\
\hline Copper & 1 & -- & -- & 92.9 & -- \\
\hline Iron & 1 & -- & -- & 1,340 & -- \\
\hline Lead & 1 & -- & -- & 9.0 & -- \\
\hline Manganese & 1 & -- & -- & 2,160 & -- \\
\hline Nickel & 1 & -- & -- & 2.1 & -- \\
\hline Zinc & 1 & -- & -- & 206 & -- \\
\hline \multicolumn{6}{|c|}{ Hydropsyche spp. } \\
\hline Arsenic & 2 & 15.7 & 14.5 & -- & -- \\
\hline Cadmium & 6 & 3.5 & .8 & 2.3 & 2.8 \\
\hline Chromium & 2 & 2.4 & 2.2 & 2.3 & -- \\
\hline Copper & 6 & 154 & 78.4 & 126 & 143 \\
\hline Iron & 6 & 1,540 & 1,190 & 1,360 & 1,360 \\
\hline Lead & 6 & 13.5 & 5.9 & 10.4 & 10.9 \\
\hline Manganese & 2 & 4,760 & 4,400 & -- & -- \\
\hline Nickel & 2 & 1.8 & 1.5 & -- & -- \\
\hline Zinc & 6 & 329 & 218 & 280 & 291 \\
\hline
\end{tabular}


Table 22. Statistical summary of biological data for the upper Clark Fork basin, Montana, August 1986 through August 2005. -Continued

[Concentrations are in micrograms per gram dry weight. Number of composite samples represents the total of all individual composite samples collected for every year that the constituent was analyzed. Values for a single sample are arbitraily listed in the "Mean" column. Because Hydropsyche insects were not sorted to the species level during 1986-89, Hydropsyche species statistics for stations sampled during those years are based on the results of all Hydropsyche species combined. At some sites, statistics of Hydropsyche morosa group are based on the combined results of two or more species. Insects collected during 1986-98 were depurated prior to analysis; depuration was discontinued in 1999. Arsenic data were not collected at some sites. Values are reported using U.S. Geological Survey rounding standards. Abbreviation: spp., one or more similar species. Symbols: <, less than minimum reporting level; --, indicates either too few samples (less than three) or insufficient data to compute statistic, or element not analyzed]

\begin{tabular}{|c|c|c|c|c|c|}
\hline Constituent & $\begin{array}{c}\text { Number of } \\
\text { composite samples }\end{array}$ & Maximum & Minimum & Mean & Median \\
\hline \multicolumn{6}{|c|}{$\begin{array}{l}461415112450801 \text { - Clark Fork below Lost Creek, near Galen } \\
\text { Period of record for biological data: } 1996-2005\end{array}$} \\
\hline \multicolumn{6}{|c|}{ Claassenia sabulosa } \\
\hline Arsenic & 1 & -- & -- & 1.5 & -- \\
\hline Cadmium & 2 & 0.4 & 0.3 & -- & -- \\
\hline Chromium & 2 & 1.9 & .4 & -- & -- \\
\hline Copper & 2 & 70.1 & 67.1 & -- & -- \\
\hline Iron & 2 & 209 & 189 & -- & -- \\
\hline Lead & 2 & 1.2 & .7 & -- & -- \\
\hline Manganese & 2 & 238 & 90.4 & -- & -- \\
\hline Nickel & 2 & .2 & $<.2$ & -- & -- \\
\hline Zinc & 2 & 245 & 208 & -- & -- \\
\hline \multicolumn{6}{|c|}{ Hydropsyche cockerelli } \\
\hline Arsenic & 7 & 11.8 & 8.8 & 11.0 & 11.4 \\
\hline Cadmium & 18 & 2.8 & 1.1 & 1.8 & 1.7 \\
\hline Chromium & 18 & 2.7 & .8 & 1.8 & 1.9 \\
\hline Copper & 18 & 147 & 48.8 & 108 & 102 \\
\hline Iron & 18 & 2,570 & 691 & 1,280 & 1,130 \\
\hline Lead & 18 & 15.2 & 4.5 & 9.6 & 8.9 \\
\hline Manganese & 18 & 3,160 & 1,230 & 1,800 & 1,670 \\
\hline Nickel & 18 & 1.9 & .9 & 1.2 & 1.1 \\
\hline Zinc & 18 & 321 & 151 & 213 & 219 \\
\hline \multicolumn{6}{|c|}{ Hydropsyche occidentalis } \\
\hline Arsenic & 3 & 15.6 & 12.7 & 13.7 & 12.9 \\
\hline Cadmium & 17 & 1.8 & .9 & 1.3 & 1.3 \\
\hline Chromium & 17 & 3.3 & 1.2 & 2.0 & 1.8 \\
\hline Copper & 17 & 157 & 52.1 & 103 & 107 \\
\hline Iron & 17 & 1,920 & 963 & 1,380 & 1,360 \\
\hline Lead & 17 & 12.4 & 6.6 & 9.5 & 9.5 \\
\hline Manganese & 17 & 3,870 & 1,220 & 2,250 & 2,150 \\
\hline Nickel & 17 & 1.7 & .9 & 1.3 & 1.4 \\
\hline Zinc & 17 & 283 & 174 & 227 & 236 \\
\hline
\end{tabular}


Table 22. Statistical summary of biological data for the upper Clark Fork basin, Montana, August 1986 through August 2005. -Continued

[Concentrations are in micrograms per gram dry weight. Number of composite samples represents the total of all individual composite samples collected for every year that the constituent was analyzed. Values for a single sample are arbitraily listed in the "Mean" column. Because Hydropsyche insects were not sorted to the species level during 1986-89, Hydropsyche species statistics for stations sampled during those years are based on the results of all Hydropsyche species combined. At some sites, statistics of Hydropsyche morosa group are based on the combined results of two or more species. Insects collected during 1986-98 were depurated prior to analysis; depuration was discontinued in 1999. Arsenic data were not collected at some sites. Values are reported using U.S. Geological Survey rounding standards. Abbreviation: spp., one or more similar species. Symbols: <, less than minimum reporting level; --, indicates either too few samples (less than three) or insufficient data to compute statistic, or element not analyzed]

\begin{tabular}{|c|c|c|c|c|c|}
\hline Constituent & $\begin{array}{l}\text { Number of } \\
\text { composite samples }\end{array}$ & Maximum & Minimum & Mean & Median \\
\hline \multicolumn{6}{|c|}{$\begin{array}{l}\text { 461415112450801—Clark Fork below Lost Creek, near Galen-Continued } \\
\text { Period of record for biological data: 1996-2005 }\end{array}$} \\
\hline \multicolumn{6}{|c|}{ Hydropsyche spp. } \\
\hline Arsenic & 1 & -- & -- & 12.0 & -- \\
\hline Cadmium & 5 & 1.8 & 1.2 & 1.5 & 1.4 \\
\hline Chromium & 5 & 2.4 & .9 & 1.5 & 1.5 \\
\hline Copper & 5 & 122 & 45.1 & 91.8 & 103 \\
\hline Iron & 5 & 1,410 & 533 & 1,110 & 1,200 \\
\hline Lead & 5 & 20.5 & 4.1 & 10.0 & 8.7 \\
\hline Manganese & 5 & 1,980 & 799 & 1,440 & 1,230 \\
\hline Nickel & 5 & 2.8 & 1.0 & 1.6 & 1.4 \\
\hline Zinc & 5 & 225 & 143 & 179 & 179 \\
\hline
\end{tabular}

461559112443301—Clark Fork at County Bridge, near Racetrack Period of record for biological data: 1996-2005

\begin{tabular}{|c|c|c|c|c|c|}
\hline \multicolumn{6}{|c|}{ Claassenia sabulosa } \\
\hline Arsenic & 0 & -- & -- & -- & -- \\
\hline Cadmium & 1 & -- & -- & 0.4 & -- \\
\hline Chromium & 1 & -- & -- & .3 & -- \\
\hline Copper & 1 & -- & -- & 40.3 & -- \\
\hline Iron & 1 & -- & -- & 113 & -- \\
\hline Lead & 1 & -- & -- & .8 & -- \\
\hline Manganese & 1 & -- & -- & 172 & -- \\
\hline Nickel & 1 & -- & -- & .2 & -- \\
\hline Zinc & 1 & -- & -- & 213 & -- \\
\hline \multicolumn{6}{|c|}{ Hydropsyche cockerelli } \\
\hline Arsenic & 6 & 14.3 & 11.1 & 12.0 & 11.6 \\
\hline Cadmium & 17 & 1.9 & .8 & 1.4 & 1.4 \\
\hline Chromium & 17 & 2.7 & .6 & 1.5 & 1.1 \\
\hline Copper & 17 & 121 & 50.0 & 85.5 & 84.3 \\
\hline Iron & 17 & 1,370 & 657 & 957 & 981 \\
\hline Lead & 17 & 10.5 & 3.7 & 6.8 & 6.9 \\
\hline Manganese & 17 & 2,020 & 646 & 1,520 & 1,690 \\
\hline Nickel & 17 & 1.4 & .7 & 1.0 & 1.0 \\
\hline Zinc & 17 & 199 & 139 & 176 & 176 \\
\hline
\end{tabular}


Table 22. Statistical summary of biological data for the upper Clark Fork basin, Montana, August 1986 through August 2005. -Continued

[Concentrations are in micrograms per gram dry weight. Number of composite samples represents the total of all individual composite samples collected for every year that the constituent was analyzed. Values for a single sample are arbitraily listed in the "Mean" column. Because Hydropsyche insects were not sorted to the species level during 1986-89, Hydropsyche species statistics for stations sampled during those years are based on the results of all Hydropsyche species combined. At some sites, statistics of Hydropsyche morosa group are based on the combined results of two or more species. Insects collected during 1986-98 were depurated prior to analysis; depuration was discontinued in 1999. Arsenic data were not collected at some sites. Values are reported using U.S. Geological Survey rounding standards. Abbreviation: spp., one or more similar species. Symbols: <, less than minimum reporting level; --, indicates either too few samples (less than three) or insufficient data to compute statistic, or element not analyzed]

\begin{tabular}{|c|c|c|c|c|c|}
\hline Constituent & $\begin{array}{l}\text { Number of } \\
\text { composite samples }\end{array}$ & Maximum & Minimum & Mean & Median \\
\hline \multicolumn{6}{|c|}{$\begin{array}{c}\text { 461559112443301_Clark Fork at County Bridge, near Racetrack-Continued } \\
\text { Period of record for biological data: 1996-2005 }\end{array}$} \\
\hline \multicolumn{6}{|c|}{ Hydropsyche occidentalis } \\
\hline Arsenic & 2 & 14.3 & 13.7 & -- & -- \\
\hline Cadmium & 15 & 2.2 & .7 & 1.4 & 1.4 \\
\hline Chromium & 15 & 3.7 & 1.1 & 2.1 & 2.0 \\
\hline Copper & 15 & 160 & 59.5 & 107 & 107 \\
\hline Iron & 15 & 1,880 & 1,030 & 1,510 & 1,520 \\
\hline Lead & 15 & 11.7 & 4.3 & 9.6 & 10.1 \\
\hline Manganese & 15 & 3,770 & 1,090 & 2,070 & 2,050 \\
\hline Nickel & 15 & 1.9 & 1.1 & 1.3 & 1.3 \\
\hline Zinc & 15 & 255 & 181 & 225 & 220 \\
\hline \multicolumn{6}{|c|}{ Hydropsyche spp. } \\
\hline Arsenic & 1 & -- & -- & 11.9 & -- \\
\hline Cadmium & 3 & 2.4 & 1.0 & 1.6 & 1.5 \\
\hline Chromium & 3 & 1.7 & .7 & 1.2 & 1.1 \\
\hline Copper & 3 & 113 & 82.9 & 93.7 & 85.2 \\
\hline Iron & 3 & 1,290 & 1,140 & 1,210 & 1,200 \\
\hline Lead & 3 & 9.6 & 5.7 & 7.5 & 7.4 \\
\hline Manganese & 3 & 1,600 & 910 & 1,210 & 1,130 \\
\hline Nickel & 3 & 1.4 & 1.1 & 1.3 & 1.3 \\
\hline Zinc & 3 & 208 & 151 & 180 & 181 \\
\hline
\end{tabular}

461903112440701—Clark Fork at Dempsey Creek Diversion, near Racetrack Period of record for biological data: 1996-2005

\begin{tabular}{|c|c|c|c|c|c|}
\hline \multicolumn{6}{|c|}{ Arctopsyche grandis } \\
\hline Arsenic & 0 & -- & -- & -- & -- \\
\hline Cadmium & 1 & -- & -- & 1.7 & -- \\
\hline Chromium & 1 & -- & -- & $<2.4$ & -- \\
\hline Copper & 1 & -- & -- & 30.8 & -- \\
\hline Iron & 1 & -- & -- & 340 & -- \\
\hline Lead & 1 & -- & -- & $<14.5$ & -- \\
\hline Manganese & 1 & -- & -- & 510 & -- \\
\hline Nickel & 1 & -- & -- & 1.0 & -- \\
\hline Zinc & 1 & -- & -- & 87 & -- \\
\hline
\end{tabular}


Table 22. Statistical summary of biological data for the upper Clark Fork basin, Montana, August 1986 through August 2005. - Continued

[Concentrations are in micrograms per gram dry weight. Number of composite samples represents the total of all individual composite samples collected for every year that the constituent was analyzed. Values for a single sample are arbitraily listed in the "Mean" column. Because Hydropsyche insects were not sorted to the species level during 1986-89, Hydropsyche species statistics for stations sampled during those years are based on the results of all Hydropsyche species combined. At some sites, statistics of Hydropsyche morosa group are based on the combined results of two or more species. Insects collected during 1986-98 were depurated prior to analysis; depuration was discontinued in 1999. Arsenic data were not collected at some sites. Values are reported using U.S. Geological Survey rounding standards. Abbreviation: spp., one or more similar species. Symbols: <, less than minimum reporting level; --, indicates either too few samples (less than three) or insufficient data to compute statistic, or element not analyzed]

\begin{tabular}{|c|c|c|c|c|c|}
\hline Constituent & $\begin{array}{l}\text { Number of } \\
\text { composite samples }\end{array}$ & Maximum & Minimum & Mean & Median \\
\hline \multicolumn{6}{|c|}{$\begin{array}{c}\text { 461903112440701 — Clark Fork at Dempsey Creek Diversion, near Racetrack—Continued } \\
\text { Period of record for biological data: 1996-2005 }\end{array}$} \\
\hline \multicolumn{6}{|c|}{ Hydropsyche cockerelli } \\
\hline Arsenic & 4 & 18.8 & 8.0 & 11.4 & 9.4 \\
\hline Cadmium & 13 & 1.7 & .7 & 1.2 & 1.3 \\
\hline Chromium & 13 & 4.0 & .4 & 1.3 & 1.0 \\
\hline Copper & 13 & 190 & 60.7 & 92.3 & 78.8 \\
\hline Iron & 13 & 2,310 & 552 & 953 & 831 \\
\hline Lead & 13 & 17.7 & 3.5 & 6.5 & 5.0 \\
\hline Manganese & 13 & 2,070 & 487 & 1,200 & 1,190 \\
\hline Nickel & 13 & 1.9 & .5 & 1.0 & .7 \\
\hline Zinc & 13 & 275 & 162 & 192 & 180 \\
\hline \multicolumn{6}{|c|}{ Hydropsyche occidentalis } \\
\hline Arsenic & 2 & 24.0 & 10.2 & -- & -- \\
\hline Cadmium & 19 & 1.8 & .7 & 1.2 & 1.2 \\
\hline Chromium & 19 & 6.2 & .8 & 2.0 & 1.8 \\
\hline Copper & 19 & 238 & 74.9 & 102 & 89.0 \\
\hline Iron & 19 & 3,390 & 940 & 1,510 & 1,500 \\
\hline Lead & 19 & 21.8 & 6.1 & 11.4 & 11.4 \\
\hline Manganese & 19 & 3,990 & 826 & 2,410 & 2,290 \\
\hline Nickel & 19 & 2.4 & 1.2 & 1.5 & 1.4 \\
\hline Zinc & 19 & 355 & 211 & 250 & 236 \\
\hline \multicolumn{6}{|c|}{ Hydropsyche spp. } \\
\hline Arsenic & 0 & -- & -- & -- & -- \\
\hline Cadmium & 2 & 1.7 & 1.6 & -- & -- \\
\hline Chromium & 2 & 2.1 & 1.4 & -- & -- \\
\hline Copper & 2 & 140 & 104 & -- & -- \\
\hline Iron & 2 & 1,610 & 1,070 & -- & -- \\
\hline Lead & 2 & 13.2 & 10.5 & -- & -- \\
\hline Manganese & 2 & 1,150 & 638 & -- & -- \\
\hline Nickel & 2 & 1.6 & 1.6 & -- & -- \\
\hline Zinc & 2 & 212 & 191 & -- & -- \\
\hline
\end{tabular}


Table 22. Statistical summary of biological data for the upper Clark Fork basin, Montana, August 1986 through August 2005. -Continued

[Concentrations are in micrograms per gram dry weight. Number of composite samples represents the total of all individual composite samples collected for every year that the constituent was analyzed. Values for a single sample are arbitraily listed in the "Mean" column. Because Hydropsyche insects were not sorted to the species level during 1986-89, Hydropsyche species statistics for stations sampled during those years are based on the results of all Hydropsyche species combined. At some sites, statistics of Hydropsyche morosa group are based on the combined results of two or more species. Insects collected during 1986-98 were depurated prior to analysis; depuration was discontinued in 1999. Arsenic data were not collected at some sites. Values are reported using U.S. Geological Survey rounding standards. Abbreviation: spp., one or more similar species. Symbols: <, less than minimum reporting level; --, indicates either too few samples (less than three) or insufficient data to compute statistic, or element not analyzed]

\begin{tabular}{|c|c|c|c|c|c|}
\hline Constituent & $\begin{array}{c}\text { Number of } \\
\text { composite samples }\end{array}$ & Maximum & Minimum & Mean & Median \\
\hline \multicolumn{6}{|c|}{$\begin{array}{l}12324200 \text { —Clark Fork at Deer Lodge } \\
\text { Period of record for biological data: 1986-87, 1990-2005 }\end{array}$} \\
\hline \multicolumn{6}{|c|}{ Arctopsyche grandis } \\
\hline Arsenic & 0 & -- & -- & -- & -- \\
\hline Cadmium & 2 & 2.4 & $<4.2$ & -- & -- \\
\hline Chromium & 2 & 1.0 & $<1.3$ & -- & -- \\
\hline Copper & 2 & 69.1 & 34.9 & -- & -- \\
\hline Iron & 2 & 676 & 537 & -- & -- \\
\hline Lead & 2 & 3.8 & $<7.8$ & -- & -- \\
\hline Manganese & 2 & 727 & 380 & -- & -- \\
\hline Nickel & 2 & $<1.7$ & $<1.3$ & -- & -- \\
\hline Zinc & 2 & 178 & 140 & -- & -- \\
\hline \multicolumn{6}{|c|}{ Hydropsyche cockerelli } \\
\hline Arsenic & 4 & 10.1 & 5.8 & 7.3 & 6.7 \\
\hline Cadmium & 27 & 2.3 & .6 & 1.3 & 1.3 \\
\hline Chromium & 27 & 3.2 & .4 & 1.6 & 1.6 \\
\hline Copper & 27 & 136 & 54.7 & 94.6 & 97.7 \\
\hline Iron & 27 & 3,340 & 490 & 1,090 & 1,040 \\
\hline Lead & 27 & 18.1 & 3.8 & 9.2 & 8.9 \\
\hline Manganese & 27 & 1,490 & 396 & 844 & 759 \\
\hline Nickel & 27 & 2.4 & .3 & 1.1 & 1.0 \\
\hline Zinc & 27 & 391 & 132 & 186 & 185 \\
\hline \multicolumn{6}{|c|}{ Hydropsyche occidentalis } \\
\hline Arsenic & 5 & 12.4 & 6.6 & 9.8 & 9.4 \\
\hline Cadmium & 42 & 2.7 & .6 & 1.3 & 1.3 \\
\hline Chromium & 42 & 3.6 & 6 & 1.9 & 1.9 \\
\hline Copper & 42 & 162 & 49.4 & 114 & 111 \\
\hline Iron & 42 & 2,060 & 557 & 1,390 & 1,420 \\
\hline Lead & 42 & 18.6 & 3.5 & 11.0 & 10.8 \\
\hline Manganese & 42 & 2,850 & 649 & 1,700 & 1,730 \\
\hline Nickel & 42 & 12.9 & 1.0 & 1.7 & 1.4 \\
\hline Zinc & 42 & 329 & 166 & 240 & 233 \\
\hline
\end{tabular}


Table 22. Statistical summary of biological data for the upper Clark Fork basin, Montana, August 1986 through August 2005. -Continued

[Concentrations are in micrograms per gram dry weight. Number of composite samples represents the total of all individual composite samples collected for every year that the constituent was analyzed. Values for a single sample are arbitraily listed in the "Mean" column. Because Hydropsyche insects were not sorted to the species level during 1986-89, Hydropsyche species statistics for stations sampled during those years are based on the results of all Hydropsyche species combined. At some sites, statistics of Hydropsyche morosa group are based on the combined results of two or more species. Insects collected during 1986-98 were depurated prior to analysis; depuration was discontinued in 1999. Arsenic data were not collected at some sites. Values are reported using U.S. Geological Survey rounding standards. Abbreviation: spp., one or more similar species. Symbols: <, less than minimum reporting level; --, indicates either too few samples (less than three) or insufficient data to compute statistic, or element not analyzed]

\begin{tabular}{|c|c|c|c|c|c|}
\hline Constituent & $\begin{array}{c}\text { Number of } \\
\text { composite samples }\end{array}$ & Maximum & Minimum & Mean & Median \\
\hline \multicolumn{6}{|c|}{$\begin{array}{l}12324200 \text {-Clark Fork at Deer Lodge-Continued } \\
\text { Period of record for biological data: 1986-87, 1990-2005 }\end{array}$} \\
\hline \multicolumn{6}{|c|}{ Hydropsyche spp. } \\
\hline Arsenic & 0 & -- & -- & -- & -- \\
\hline Cadmium & 3 & 2.6 & 2.0 & 2.4 & 2.5 \\
\hline Chromium & 0 & -- & -- & -- & -- \\
\hline Copper & 3 & 222 & 175 & 191 & 177 \\
\hline Iron & 3 & 2,220 & 1,850 & 2,010 & 1,950 \\
\hline Lead & 3 & 16.7 & 15.0 & 16.1 & 16.7 \\
\hline Manganese & 0 & -- & -- & -- & -- \\
\hline Nickel & 0 & -- & -- & -- & -- \\
\hline Zinc & 3 & 298 & 197 & 257 & 276 \\
\hline \multicolumn{6}{|c|}{$\begin{array}{l}12324590 \text { —Little Blackfoot River near Garrison } \\
\text { Period of record for biological data: 1987, 1994, 1998, 2001, } 2004\end{array}$} \\
\hline \multicolumn{6}{|c|}{ Arctopsyche grandis } \\
\hline Arsenic & 3 & 4.2 & 3.2 & 3.6 & 3.3 \\
\hline Cadmium & 18 & .7 & .2 & .4 & .4 \\
\hline Chromium & 18 & 1.6 & .6 & .9 & .8 \\
\hline Copper & 18 & 14.2 & 9.0 & 11.9 & 11.9 \\
\hline Iron & 18 & 716 & 177 & 419 & 414 \\
\hline Lead & 18 & 1.3 & .5 & .8 & .8 \\
\hline Manganese & 18 & 1,140 & 318 & 733 & 719 \\
\hline Nickel & 18 & 1.4 & .4 & .8 & .8 \\
\hline Zinc & 18 & 214 & 113 & 162 & 160 \\
\hline \multicolumn{6}{|c|}{ Claassenia sabulosa } \\
\hline Arsenic & 1 & -- & -- & 1.3 & -- \\
\hline Cadmium & 8 & 0.5 & 0.1 & .2 & 0.2 \\
\hline Chromium & 8 & .9 & .3 & .6 & .7 \\
\hline Copper & 8 & 37.2 & 20.0 & 30.4 & 30.9 \\
\hline Iron & 8 & 319 & 98.4 & 178 & 178 \\
\hline Lead & 8 & $<.8$ & $<.1$ & ${ }^{1} .4$ & ${ }^{1} .3$ \\
\hline Manganese & 8 & 149 & 46.7 & 75.9 & 62.1 \\
\hline Nickel & 8 & .7 & .4 & .5 & .5 \\
\hline Zinc & 8 & 271 & 172 & 211 & 206 \\
\hline
\end{tabular}


Table 22. Statistical summary of biological data for the upper Clark Fork basin, Montana, August 1986 through August 2005. -Continued

[Concentrations are in micrograms per gram dry weight. Number of composite samples represents the total of all individual composite samples collected for every year that the constituent was analyzed. Values for a single sample are arbitraily listed in the "Mean" column. Because Hydropsyche insects were not sorted to the species level during 1986-89, Hydropsyche species statistics for stations sampled during those years are based on the results of all Hydropsyche species combined. At some sites, statistics of Hydropsyche morosa group are based on the combined results of two or more species. Insects collected during 1986-98 were depurated prior to analysis; depuration was discontinued in 1999. Arsenic data were not collected at some sites. Values are reported using U.S. Geological Survey rounding standards. Abbreviation: spp., one or more similar species. Symbols: <, less than minimum reporting level; --, indicates either too few samples (less than three) or insufficient data to compute statistic, or element not analyzed]

\begin{tabular}{|c|c|c|c|c|c|}
\hline Constituent & $\begin{array}{c}\text { Number of } \\
\text { composite samples }\end{array}$ & Maximum & Minimum & Mean & Median \\
\hline \multicolumn{6}{|c|}{$\begin{array}{l}12324590 \text { - Little Blackfoot River near Garrison-Continued } \\
\text { Period of record for biological data: 1987, 1994, 1998, 2001, } 2004\end{array}$} \\
\hline \multicolumn{6}{|c|}{ Hydropsyche cockerelli } \\
\hline Arsenic & 0 & -- & -- & -- & -- \\
\hline Cadmium & 1 & -- & -- & 0.6 & -- \\
\hline Chromium & 1 & -- & -- & 1.6 & -- \\
\hline Copper & 1 & -- & -- & 28.4 & -- \\
\hline Iron & 1 & -- & -- & 478 & -- \\
\hline Lead & 1 & -- & -- & 3.6 & -- \\
\hline Manganese & 1 & -- & -- & 399 & -- \\
\hline Nickel & 1 & -- & -- & 1.2 & -- \\
\hline Zinc & 1 & -- & -- & 123 & -- \\
\hline \multicolumn{6}{|c|}{ Hydropsyche occidentalis } \\
\hline Arsenic & 0 & -- & -- & -- & -- \\
\hline Cadmium & 2 & 0.3 & $<0.7$ & -- & -- \\
\hline Chromium & 2 & 2.3 & 1.3 & -- & -- \\
\hline Copper & 2 & 15.2 & 15.1 & -- & -- \\
\hline Iron & 2 & 1,340 & 426 & -- & -- \\
\hline Lead & 2 & 2.3 & $<3.7$ & -- & -- \\
\hline Manganese & 2 & 554 & 434 & -- & -- \\
\hline Nickel & 2 & 1.1 & .8 & -- & -- \\
\hline Zinc & 2 & 137 & 110 & -- & -- \\
\hline \multicolumn{6}{|c|}{ Hydropsyche spp. } \\
\hline Arsenic & 1 & -- & -- & 3.7 & -- \\
\hline Cadmium & 1 & -- & -- & $<.2$ & -- \\
\hline Chromium & 1 & -- & -- & 1.8 & -- \\
\hline Copper & 1 & -- & -- & 11.1 & -- \\
\hline Iron & 1 & -- & -- & 1,000 & -- \\
\hline Lead & 1 & -- & -- & $<2.4$ & -- \\
\hline Manganese & 1 & -- & -- & 1,200 & -- \\
\hline Nickel & 1 & -- & -- & .9 & -- \\
\hline Zinc & 1 & -- & -- & 151 & -- \\
\hline
\end{tabular}


Table 22. Statistical summary of biological data for the upper Clark Fork basin, Montana, August 1986 through August 2005. -Continued

[Concentrations are in micrograms per gram dry weight. Number of composite samples represents the total of all individual composite samples collected for every year that the constituent was analyzed. Values for a single sample are arbitraily listed in the "Mean" column. Because Hydropsyche insects were not sorted to the species level during 1986-89, Hydropsyche species statistics for stations sampled during those years are based on the results of all Hydropsyche species combined. At some sites, statistics of Hydropsyche morosa group are based on the combined results of two or more species. Insects collected during 1986-98 were depurated prior to analysis; depuration was discontinued in 1999. Arsenic data were not collected at some sites. Values are reported using U.S. Geological Survey rounding standards. Abbreviation: spp., one or more similar species. Symbols: <, less than minimum reporting level; --, indicates either too few samples (less than three) or insufficient data to compute statistic, or element not analyzed]

\begin{tabular}{|c|c|c|c|c|c|}
\hline Constituent & $\begin{array}{c}\text { Number of } \\
\text { composite samples }\end{array}$ & Maximum & Minimum & Mean & Median \\
\hline \multicolumn{6}{|c|}{$\begin{array}{l}12324680 \text { - Clark Fork at Goldcreek } \\
\text { Period of record for biological data: 1992-2005 }\end{array}$} \\
\hline \multicolumn{6}{|c|}{ Arctopsyche grandis } \\
\hline Arsenic & 13 & 6.4 & 1.8 & 4.1 & 3.3 \\
\hline Cadmium & 42 & 6.6 & .6 & 2.0 & 1.8 \\
\hline Chromium & 42 & 3.3 & .4 & 1.4 & 1.1 \\
\hline Copper & 42 & 129 & 19.9 & 44.3 & 37.5 \\
\hline Iron & 42 & 2,360 & 195 & 686 & 517 \\
\hline Lead & 42 & 10.9 & 1.1 & 3.6 & 3.4 \\
\hline Manganese & 42 & 1,580 & 436 & 835 & 789 \\
\hline Nickel & 42 & 1.8 & .2 & .7 & .6 \\
\hline Zinc & 42 & 326 & 149 & 199 & 183 \\
\hline \multicolumn{6}{|c|}{ Claassenia sabulosa } \\
\hline Arsenic & 8 & 2.1 & 0.7 & 1.3 & 1.2 \\
\hline Cadmium & 28 & 3.5 & .1 & 1.0 & .7 \\
\hline Chromium & 28 & 1.6 & .2 & .6 & .5 \\
\hline Copper & 28 & 81.7 & 33.0 & 56.6 & 56.3 \\
\hline Iron & 28 & 567 & 63.0 & 193 & 189 \\
\hline Lead & 28 & 1.8 & .4 & .9 & .9 \\
\hline Manganese & 28 & 320 & 50.6 & 138 & 113 \\
\hline Nickel & 28 & .7 & .1 & .3 & .3 \\
\hline Zinc & 28 & 351 & 166 & 263 & 260 \\
\hline \multicolumn{6}{|c|}{ Hydropsyche cockerelli } \\
\hline Arsenic & 8 & 6.1 & 4.1 & 5.2 & 5.4 \\
\hline Cadmium & 27 & 2.6 & .5 & 1.3 & 1.3 \\
\hline Chromium & 27 & 4.7 & .5 & 2.2 & 2.0 \\
\hline Copper & 27 & 188 & 17.1 & 69.9 & 58.4 \\
\hline Iron & 27 & 3,250 & 522 & 1,150 & 930 \\
\hline Lead & 27 & 16.2 & 2.4 & 6.6 & 5.3 \\
\hline Manganese & 27 & 1,670 & 538 & 913 & 906 \\
\hline Nickel & 27 & 2.3 & .3 & 1.2 & 1.0 \\
\hline Zinc & 27 & 249 & 106 & 183 & 184 \\
\hline
\end{tabular}


Table 22. Statistical summary of biological data for the upper Clark Fork basin, Montana, August 1986 through August 2005. -Continued

[Concentrations are in micrograms per gram dry weight. Number of composite samples represents the total of all individual composite samples collected for every year that the constituent was analyzed. Values for a single sample are arbitraily listed in the "Mean" column. Because Hydropsyche insects were not sorted to the species level during 1986-89, Hydropsyche species statistics for stations sampled during those years are based on the results of all Hydropsyche species combined. At some sites, statistics of Hydropsyche morosa group are based on the combined results of two or more species. Insects collected during 1986-98 were depurated prior to analysis; depuration was discontinued in 1999. Arsenic data were not collected at some sites. Values are reported using U.S. Geological Survey rounding standards. Abbreviation: spp., one or more similar species. Symbols: <, less than minimum reporting level; --, indicates either too few samples (less than three) or insufficient data to compute statistic, or element not analyzed]

\begin{tabular}{|c|c|c|c|c|c|}
\hline Constituent & $\begin{array}{l}\text { Number of } \\
\text { composite samples }\end{array}$ & Maximum & Minimum & Mean & Median \\
\hline \multicolumn{6}{|c|}{$\begin{array}{l}12324680 \text {-Clark Fork at Goldcreek-Continued } \\
\text { Period of record for biological data: } 1992-2005\end{array}$} \\
\hline \multicolumn{6}{|c|}{ Hydropsyche morosa group } \\
\hline Arsenic & 0 & -- & -- & -- & -- \\
\hline Cadmium & 4 & 1.7 & 1.1 & 1.4 & 1.4 \\
\hline Chromium & 4 & 1.4 & 1.3 & 1.4 & 1.4 \\
\hline Copper & 4 & 72.9 & 43.8 & 60.5 & 62.7 \\
\hline Iron & 4 & 1,320 & 612 & 1,050 & 1,130 \\
\hline Lead & 4 & 6.9 & 2.4 & 4.6 & 4.6 \\
\hline Manganese & 4 & 1,030 & 538 & 804 & 822 \\
\hline Nickel & 4 & 1.4 & .9 & 1.2 & 1.2 \\
\hline Zinc & 4 & 190 & 137 & 167 & 170 \\
\hline \multicolumn{6}{|c|}{ Hydropsyche occidentalis } \\
\hline Arsenic & 3 & 5.8 & 4.7 & 5.3 & 5.3 \\
\hline Cadmium & 18 & 1.7 & .4 & 1.2 & 1.3 \\
\hline Chromium & 18 & 3.9 & .4 & 1.6 & 1.7 \\
\hline Copper & 18 & 156 & 26.4 & 62.8 & 58.0 \\
\hline Iron & 18 & 2,720 & 466 & 1,140 & 1,070 \\
\hline Lead & 18 & 15.7 & 2.9 & 6.9 & 5.8 \\
\hline Manganese & 18 & 2,210 & 530 & 1,210 & 1,080 \\
\hline Nickel & 18 & 2.5 & .8 & 1.2 & 1.0 \\
\hline Zinc & 18 & 277 & 97 & 195 & 196 \\
\hline
\end{tabular}

12331800 - Clark Fork near Drummond

Period of record for biological data: 1986, 1991-2005

\begin{tabular}{lccccc}
\hline \multicolumn{5}{c}{ Arctopsyche grandis } \\
\hline Arsenic & 9 & 4.4 & 2.4 & 3.3 & 3.3 \\
Cadmium & 41 & 3.8 & .4 & 1.3 & 1.1 \\
Chromium & 41 & 2.5 & .2 & 1.0 & 1.0 \\
Copper & 41 & 89.2 & 16.9 & 32.4 & 27.4 \\
Iron & 41 & 1,660 & 240 & 568 & 511 \\
Lead & 41 & 11.8 & 2.1 & 4.4 & 3.9 \\
Manganese & 41 & 2,010 & 456 & .7 & 733 \\
Nickel & 41 & 1.9 & 140 & .2 & .6 \\
Zinc & 41 & 308 & & 188 & 183
\end{tabular}


Table 22. Statistical summary of biological data for the upper Clark Fork basin, Montana, August 1986 through August 2005. -Continued

[Concentrations are in micrograms per gram dry weight. Number of composite samples represents the total of all individual composite samples collected for every year that the constituent was analyzed. Values for a single sample are arbitraily listed in the "Mean" column. Because Hydropsyche insects were not sorted to the species level during 1986-89, Hydropsyche species statistics for stations sampled during those years are based on the results of all Hydropsyche species combined. At some sites, statistics of Hydropsyche morosa group are based on the combined results of two or more species. Insects collected during 1986-98 were depurated prior to analysis; depuration was discontinued in 1999. Arsenic data were not collected at some sites. Values are reported using U.S. Geological Survey rounding standards. Abbreviation: spp., one or more similar species. Symbols: <, less than minimum reporting level; --, indicates either too few samples (less than three) or insufficient data to compute statistic, or element not analyzed]

\begin{tabular}{|c|c|c|c|c|c|}
\hline Constituent & $\begin{array}{c}\text { Number of } \\
\text { composite samples }\end{array}$ & Maximum & Minimum & Mean & Median \\
\hline \multicolumn{6}{|c|}{$\begin{array}{l}12331800 \text {-Clark Fork near Drummond-Continued } \\
\text { Period of record for biological data: 1986, 1991-2005 }\end{array}$} \\
\hline \multicolumn{6}{|c|}{ Claassenia sabulosa } \\
\hline Arsenic & 6 & 1.3 & 0.7 & 1.1 & 1.2 \\
\hline Cadmium & 42 & 2.8 & .1 & 1.1 & 1.0 \\
\hline Chromium & 42 & 3.3 & .1 & .7 & .6 \\
\hline Copper & 42 & 165 & 18.0 & 63.5 & 53.8 \\
\hline Iron & 42 & 387 & 45.4 & 158 & 135 \\
\hline Lead & 42 & 2.9 & .2 & .9 & .8 \\
\hline Manganese & 42 & 410 & 33.1 & 161 & 140 \\
\hline Nickel & 42 & 1.1 & .1 & .3 & .2 \\
\hline Zinc & 42 & 567 & 103 & 273 & 256 \\
\hline \multicolumn{6}{|c|}{ Hydropsyche cockerelli } \\
\hline Arsenic & 6 & 5.7 & 3.9 & 4.5 & 4.3 \\
\hline Cadmium & 35 & 2.3 & .3 & 1.1 & .8 \\
\hline Chromium & 35 & 3.5 & .4 & 1.6 & 1.5 \\
\hline Copper & 35 & 156 & 30.0 & 58.1 & 50.3 \\
\hline Iron & 35 & 2,500 & 506 & 1,150 & 969 \\
\hline Lead & 35 & 15.0 & 5.1 & 8.2 & 7.4 \\
\hline Manganese & 35 & 1,680 & 549 & 977 & 913 \\
\hline Nickel & 35 & 2.0 & .5 & 1.1 & 1.0 \\
\hline Zinc & 35 & 248 & 134 & 192 & 186 \\
\hline \multicolumn{6}{|c|}{ Hydropsyche morosa group } \\
\hline Arsenic & 0 & -- & -- & -- & -- \\
\hline Cadmium & 6 & 1.3 & 1.1 & 1.2 & 1.2 \\
\hline Chromium & 6 & 2.8 & 1.9 & 2.3 & 2.2 \\
\hline Copper & 6 & 57.4 & 50.2 & 55.2 & 55.8 \\
\hline Iron & 6 & 1,730 & 1,370 & 1,570 & 1,600 \\
\hline Lead & 6 & 10.8 & 7.0 & 8.9 & 9.0 \\
\hline Manganese & 6 & 1,940 & 1,260 & 1,610 & 1,610 \\
\hline Nickel & 6 & 1.7 & 1.3 & 1.5 & 1.5 \\
\hline Zinc & 6 & 250 & 227 & 239 & 240 \\
\hline
\end{tabular}


Table 22. Statistical summary of biological data for the upper Clark Fork basin, Montana, August 1986 through August 2005. -Continued

[Concentrations are in micrograms per gram dry weight. Number of composite samples represents the total of all individual composite samples collected for every year that the constituent was analyzed. Values for a single sample are arbitraily listed in the "Mean" column. Because Hydropsyche insects were not sorted to the species level during 1986-89, Hydropsyche species statistics for stations sampled during those years are based on the results of all Hydropsyche species combined. At some sites, statistics of Hydropsyche morosa group are based on the combined results of two or more species. Insects collected during 1986-98 were depurated prior to analysis; depuration was discontinued in 1999. Arsenic data were not collected at some sites. Values are reported using U.S. Geological Survey rounding standards. Abbreviation: spp., one or more similar species. Symbols: <, less than minimum reporting level; --, indicates either too few samples (less than three) or insufficient data to compute statistic, or element not analyzed]

\begin{tabular}{|c|c|c|c|c|c|}
\hline Constituent & $\begin{array}{c}\text { Number of } \\
\text { composite samples }\end{array}$ & Maximum & Minimum & Mean & Median \\
\hline \multicolumn{6}{|c|}{$\begin{array}{l}12331800 \text {-Clark Fork near Drummond-Continued } \\
\text { Period of record for biological data: 1986, 1991-2005 }\end{array}$} \\
\hline \multicolumn{6}{|c|}{ Hydropsyche occidentalis } \\
\hline Arsenic & 5 & 6.9 & 4.3 & 5.0 & 4.6 \\
\hline Cadmium & 21 & 2.0 & .4 & 1.0 & 1.0 \\
\hline Chromium & 21 & 8.1 & .4 & 2.3 & 2.2 \\
\hline Copper & 21 & 118 & 13.3 & 54.1 & 55.0 \\
\hline Iron & 21 & 2,060 & 424 & 1,250 & 1,180 \\
\hline Lead & 21 & 14.0 & 2.9 & 8.7 & 8.7 \\
\hline Manganese & 21 & 2,920 & 619 & 1,500 & 1,200 \\
\hline Nickel & 21 & 2.4 & .5 & 1.4 & 1.2 \\
\hline Zinc & 21 & 293 & 157 & 220 & 221 \\
\hline \multicolumn{6}{|c|}{ Hydropsyche spp. } \\
\hline Arsenic & 0 & -- & -- & -- & -- \\
\hline Cadmium & 1 & -- & -- & 2.6 & -- \\
\hline Chromium & 0 & -- & -- & -- & -- \\
\hline Copper & 1 & -- & -- & 85.0 & -- \\
\hline Iron & 1 & -- & -- & 913 & -- \\
\hline Lead & 1 & -- & -- & 9.1 & -- \\
\hline Manganese & 0 & -- & -- & -- & -- \\
\hline Nickel & 0 & -- & -- & -- & -- \\
\hline Zinc & 1 & -- & -- & 260 & -- \\
\hline \multicolumn{6}{|c|}{$\begin{array}{l}\text { 12334510-Rock Creek near Clinton } \\
\text { Period of record for biological data: 1987; 1991-1999; 2001-2005 }\end{array}$} \\
\hline \multicolumn{6}{|c|}{ Arctopsyche grandis } \\
\hline Arsenic & 8 & 2.5 & 1.5 & 2.0 & 2.0 \\
\hline Cadmium & 46 & .4 & $<.1$ & ${ }^{1} .2$ & ${ }^{1} .2$ \\
\hline Chromium & 46 & 2.9 & .4 & 1.1 & 1.0 \\
\hline Copper & 46 & 15.7 & 4.7 & 8.6 & 8.5 \\
\hline Iron & 46 & 1,090 & 191 & 585 & 531 \\
\hline Lead & 46 & 1.1 & $<.1$ & ${ }^{1} .4$ & ${ }^{1} .4$ \\
\hline Manganese & 46 & 454 & 113 & 251 & 245 \\
\hline Nickel & 46 & 1.8 & .2 & .9 & .9 \\
\hline Zinc & 46 & 189 & 83.9 & 127 & 130 \\
\hline
\end{tabular}


Table 22. Statistical summary of biological data for the upper Clark Fork basin, Montana, August 1986 through August 2005. -Continued

[Concentrations are in micrograms per gram dry weight. Number of composite samples represents the total of all individual composite samples collected for every year that the constituent was analyzed. Values for a single sample are arbitraily listed in the "Mean" column. Because Hydropsyche insects were not sorted to the species level during 1986-89, Hydropsyche species statistics for stations sampled during those years are based on the results of all Hydropsyche species combined. At some sites, statistics of Hydropsyche morosa group are based on the combined results of two or more species. Insects collected during 1986-98 were depurated prior to analysis; depuration was discontinued in 1999. Arsenic data were not collected at some sites. Values are reported using U.S. Geological Survey rounding standards. Abbreviation: spp., one or more similar species. Symbols: <, less than minimum reporting level; --, indicates either too few samples (less than three) or insufficient data to compute statistic, or element not analyzed]

\begin{tabular}{|c|c|c|c|c|c|}
\hline Constituent & $\begin{array}{l}\text { Number of } \\
\text { composite samples }\end{array}$ & Maximum & Minimum & Mean & Median \\
\hline \multicolumn{6}{|c|}{$\begin{array}{l}12334510 \text { —Rock Creek near Clinton-Continued } \\
\text { Period of record for biological data: 1987; 1991-1999; 2001-2005 }\end{array}$} \\
\hline \multicolumn{6}{|c|}{ Claassenia sabulosa } \\
\hline Arsenic & 4 & 1.1 & 1.0 & 1.0 & 1.0 \\
\hline Cadmium & 23 & .3 & $<.1$ & ${ }^{1} .2$ & ${ }^{1} .1$ \\
\hline Chromium & 23 & 1.8 & .1 & .6 & .5 \\
\hline Copper & 23 & 40.7 & 18.1 & 28.7 & 28.5 \\
\hline Iron & 23 & 129 & 49.8 & 93.8 & 102 \\
\hline Lead & 23 & 1.0 & .1 & .4 & .3 \\
\hline Manganese & 23 & 76.3 & 15.7 & 36.6 & 34.8 \\
\hline Nickel & 23 & .9 & .1 & .3 & .3 \\
\hline Zinc & 23 & 264 & 139 & 194 & 190 \\
\hline \multicolumn{6}{|c|}{ Hydropsyche cockerelli } \\
\hline Arsenic & 1 & -- & -- & 2.4 & -- \\
\hline Cadmium & 4 & 0.3 & $<0.2$ & ${ }^{1} .2$ & ${ }^{1}<0.2$ \\
\hline Chromium & 4 & 1.0 & .3 & .8 & .9 \\
\hline Copper & 4 & 13.1 & 6.0 & 8.5 & 7.4 \\
\hline Iron & 4 & 825 & 485 & 604 & 553 \\
\hline Lead & 4 & $<1.1$ & .4 & ${ }^{1} .5$ & ${ }^{1}<1.1$ \\
\hline Manganese & 4 & 266 & 192 & 231 & 233 \\
\hline Nickel & 4 & 1.0 & .4 & .6 & .6 \\
\hline Zinc & 4 & 99 & 82 & 91 & 91 \\
\hline \multicolumn{6}{|c|}{ Hydropsyche occidentalis } \\
\hline Arsenic & 1 & -- & -- & 2.2 & -- \\
\hline Cadmium & 5 & 0.4 & $<0.3$ & ${ }^{1} .2$ & ${ }^{1} 0.1$ \\
\hline Chromium & 5 & 2.4 & .9 & 1.6 & 1.6 \\
\hline Copper & 5 & 17.6 & 5.1 & 10.6 & 10.2 \\
\hline Iron & 5 & 973 & 520 & 709 & 652 \\
\hline Lead & 5 & 6.0 & 1.2 & 3.0 & 1.8 \\
\hline Manganese & 5 & 295 & 169 & 242 & 262 \\
\hline Nickel & 5 & 1.7 & .6 & 1.2 & 1.4 \\
\hline Zinc & 5 & 144 & 99 & 116 & 117 \\
\hline
\end{tabular}


Table 22. Statistical summary of biological data for the upper Clark Fork basin, Montana, August 1986 through August 2005. -Continued

[Concentrations are in micrograms per gram dry weight. Number of composite samples represents the total of all individual composite samples collected for every year that the constituent was analyzed. Values for a single sample are arbitraily listed in the "Mean" column. Because Hydropsyche insects were not sorted to the species level during 1986-89, Hydropsyche species statistics for stations sampled during those years are based on the results of all Hydropsyche species combined. At some sites, statistics of Hydropsyche morosa group are based on the combined results of two or more species. Insects collected during 1986-98 were depurated prior to analysis; depuration was discontinued in 1999. Arsenic data were not collected at some sites. Values are reported using U.S. Geological Survey rounding standards. Abbreviation: spp., one or more similar species. Symbols: <, less than minimum reporting level; --, indicates either too few samples (less than three) or insufficient data to compute statistic, or element not analyzed]

\begin{tabular}{|c|c|c|c|c|c|}
\hline Constituent & $\begin{array}{c}\text { Number of } \\
\text { composite samples }\end{array}$ & Maximum & Minimum & Mean & Median \\
\hline \multicolumn{6}{|c|}{$\begin{array}{l}12334510 \text {-Rock Creek near Clinton-Continued } \\
\text { Period of record for biological data: 1987; 1991-1999; 2001-2005 }\end{array}$} \\
\hline \multicolumn{6}{|c|}{ Hydropsyche spp. } \\
\hline Arsenic & 1 & -- & -- & 0.7 & -- \\
\hline Cadmium & 4 & 0.3 & $<0.5$ & ${ }^{1} .2$ & ${ }^{1} 0.2$ \\
\hline Chromium & 4 & 2.1 & 1.1 & 1.6 & 1.7 \\
\hline Copper & 4 & 16.2 & 6.1 & 12.2 & 13.3 \\
\hline Iron & 4 & 1,140 & 789 & 949 & 932 \\
\hline Lead & 4 & $<4.9$ & $<1.8$ & $1--$ & ${ }^{1}<2.9$ \\
\hline Manganese & 4 & 462 & 299 & 394 & 407 \\
\hline Nickel & 4 & 1.3 & .8 & 1.1 & 1.1 \\
\hline Zinc & 4 & 135 & 112 & 123 & 121 \\
\hline
\end{tabular}

12334550 - Clark Fork at Turah Bridge, near Bonner Period of record for biological data: 1986; 1991-2005

\begin{tabular}{|c|c|c|c|c|c|}
\hline \multicolumn{6}{|c|}{ Arctopsyche grandis } \\
\hline Arsenic & 11 & 5.0 & 3.1 & 4.2 & 4.2 \\
\hline Cadmium & 53 & 2.7 & .3 & 1.2 & .8 \\
\hline Chromium & 53 & 4.1 & .5 & 1.6 & 1.4 \\
\hline Copper & 53 & 125 & 20.1 & 37.1 & 29.2 \\
\hline Iron & 53 & 2,870 & 372 & 915 & 712 \\
\hline Lead & 53 & 13.2 & 1.6 & 4.2 & 3.2 \\
\hline Manganese & 53 & 902 & 324 & 636 & 643 \\
\hline Nickel & 53 & 2.7 & .4 & 1.1 & .9 \\
\hline Zinc & 53 & 276 & 111 & 196 & 196 \\
\hline \multicolumn{6}{|c|}{ Claassenia sabulosa } \\
\hline Arsenic & 7 & 1.9 & 0.8 & 1.2 & 1.1 \\
\hline Cadmium & 33 & 2.5 & .1 & 1.0 & .8 \\
\hline Chromium & 33 & 2.0 & .2 & .7 & 6 \\
\hline Copper & 33 & 87.6 & 37.5 & 57.0 & 53.4 \\
\hline Iron & 33 & 340 & 58.6 & 116 & 105 \\
\hline Lead & 33 & 1.6 & .2 & .6 & 6 \\
\hline Manganese & 33 & 215 & 37.2 & 85.7 & 74.5 \\
\hline Nickel & 33 & .6 & .1 & .2 & .2 \\
\hline Zinc & 33 & 284 & 144 & 224 & 230 \\
\hline
\end{tabular}


Table 22. Statistical summary of biological data for the upper Clark Fork basin, Montana, August 1986 through August 2005. -Continued

[Concentrations are in micrograms per gram dry weight. Number of composite samples represents the total of all individual composite samples collected for every year that the constituent was analyzed. Values for a single sample are arbitraily listed in the "Mean" column. Because Hydropsyche insects were not sorted to the species level during 1986-89, Hydropsyche species statistics for stations sampled during those years are based on the results of all Hydropsyche species combined. At some sites, statistics of Hydropsyche morosa group are based on the combined results of two or more species. Insects collected during 1986-98 were depurated prior to analysis; depuration was discontinued in 1999. Arsenic data were not collected at some sites. Values are reported using U.S. Geological Survey rounding standards. Abbreviation: spp., one or more similar species. Symbols: <, less than minimum reporting level; --, indicates either too few samples (less than three) or insufficient data to compute statistic, or element not analyzed]

\begin{tabular}{|c|c|c|c|c|c|}
\hline Constituent & $\begin{array}{c}\text { Number of } \\
\text { composite samples }\end{array}$ & Maximum & Minimum & Mean & Median \\
\hline \multicolumn{6}{|c|}{$\begin{array}{l}\text { 12334550 —Clark Fork at Turah Bridge, near Bonner-Continued } \\
\text { Period of record for biological data: 1986; 1991-2005 }\end{array}$} \\
\hline \multicolumn{6}{|c|}{ Hydropsyche cockerelli } \\
\hline Arsenic & 7 & 5.1 & 3.7 & 4.2 & 4.0 \\
\hline Cadmium & 35 & 1.8 & .3 & .8 & .7 \\
\hline Chromium & 35 & 8.0 & .2 & 1.8 & 1.6 \\
\hline Copper & 35 & 118 & 26.4 & 46.9 & 41.8 \\
\hline Iron & 35 & 2,530 & 566 & 1,180 & 1,060 \\
\hline Lead & 35 & 12.1 & 2.2 & 5.2 & 4.9 \\
\hline Manganese & 35 & 805 & 426 & 617 & 589 \\
\hline Nickel & 35 & 2.6 & 6 & 1.2 & 1.2 \\
\hline Zinc & 35 & 228 & 119 & 183 & 180 \\
\hline \multicolumn{6}{|c|}{ Hydropsyche morosa group } \\
\hline Arsenic & 0 & -- & -- & -- & -- \\
\hline Cadmium & 2 & 1.3 & 1.1 & -- & -- \\
\hline Chromium & 2 & 4.6 & 2.4 & -- & -- \\
\hline Copper & 2 & 84.1 & 26.8 & -- & -- \\
\hline Iron & 2 & 1,800 & 986 & -- & -- \\
\hline Lead & 2 & 6.6 & $<7.8$ & -- & -- \\
\hline Manganese & 2 & 1,320 & 537 & -- & -- \\
\hline Nickel & 2 & 1.7 & 1.3 & -- & -- \\
\hline Zinc & 2 & 231 & 171 & -- & -- \\
\hline \multicolumn{6}{|c|}{ Hydropsyche occidentalis } \\
\hline Arsenic & 6 & 5.9 & 3.6 & 4.3 & 4.1 \\
\hline Cadmium & 26 & 1.8 & .3 & .9 & .8 \\
\hline Chromium & 26 & 3.2 & .6 & 1.7 & 1.5 \\
\hline Copper & 26 & 102 & 27.4 & 47.4 & 42.2 \\
\hline Iron & 26 & 2,310 & 472 & 1,180 & 1,130 \\
\hline Lead & 26 & 14.2 & 3.0 & 6.4 & 5.7 \\
\hline Manganese & 26 & 1,600 & 454 & 857 & 744 \\
\hline Nickel & 26 & 3.2 & .6 & 1.2 & 1.1 \\
\hline Zinc & 26 & 416 & 145 & 211 & 222 \\
\hline
\end{tabular}


Table 22. Statistical summary of biological data for the upper Clark Fork basin, Montana, August 1986 through August 2005. -Continued

[Concentrations are in micrograms per gram dry weight. Number of composite samples represents the total of all individual composite samples collected for every year that the constituent was analyzed. Values for a single sample are arbitraily listed in the "Mean" column. Because Hydropsyche insects were not sorted to the species level during 1986-89, Hydropsyche species statistics for stations sampled during those years are based on the results of all Hydropsyche species combined. At some sites, statistics of Hydropsyche morosa group are based on the combined results of two or more species. Insects collected during 1986-98 were depurated prior to analysis; depuration was discontinued in 1999. Arsenic data were not collected at some sites. Values are reported using U.S. Geological Survey rounding standards. Abbreviation: spp., one or more similar species. Symbols: <, less than minimum reporting level; --, indicates either too few samples (less than three) or insufficient data to compute statistic, or element not analyzed]

\begin{tabular}{|c|c|c|c|c|c|}
\hline Constituent & $\begin{array}{c}\text { Number of } \\
\text { composite samples }\end{array}$ & Maximum & Minimum & Mean & Median \\
\hline
\end{tabular}

12334550 - Clark Fork at Turah Bridge, near Bonner-Continued Period of record for biological data: 1986; 1991-2005

\begin{tabular}{llllll}
\hline \multicolumn{5}{c}{ Hydropsyche spp. } \\
\hline Arsenic & 0 & -- & -- & - & - \\
Cadmium & 1 & -- & -- & 1.3 & - \\
Chromium & 1 & -- & -- & 2.4 & - \\
Copper & 1 & -- & -- & 84.1 & - \\
Iron & 1 & -- & -- & 1,800 & - \\
Lead & 1 & -- & -- & $<7.8$ & - \\
Manganese & 1 & -- & -- & 537 & - \\
Nickel & 1 & -- & -- & 1.3 & - \\
Zinc & 1 & -- & -- & 171 & - \\
\hline
\end{tabular}

12340000 - Blackfoot River near Bonner

Period of record for biological data: 1986, 1987, 1991, 1993, 1996, 1998, 2000, 2003

\begin{tabular}{|c|c|c|c|c|c|}
\hline \multicolumn{6}{|c|}{ Arctopsyche grandis } \\
\hline Arsenic & 1 & -- & -- & 2.8 & -- \\
\hline Cadmium & 11 & 0.4 & $<0.1$ & ${ }^{1} .2$ & ${ }^{1} 0.2$ \\
\hline Chromium & 6 & 1.8 & .8 & 1.2 & 1.2 \\
\hline Copper & 11 & 13.4 & 9.9 & 12.0 & 12.0 \\
\hline Iron & 11 & 1,230 & 108 & 606 & 617 \\
\hline Lead & 11 & 2.3 & .5 & 1.0 & 6 \\
\hline Manganese & 6 & 517 & 286 & 404 & 393 \\
\hline Nickel & 6 & 1.2 & .7 & .9 & .9 \\
\hline Zinc & 11 & 143 & 123 & 135 & 136 \\
\hline \multicolumn{6}{|c|}{ Claassenia sabulosa } \\
\hline Arsenic & 0 & -- & -- & -- & -- \\
\hline Cadmium & 11 & 0.2 & 0.1 & 0.1 & 0.1 \\
\hline Chromium & 6 & .9 & .3 & .5 & .5 \\
\hline Copper & 11 & 88.5 & 19.0 & 45.2 & 44.0 \\
\hline Iron & 11 & 158 & 46.2 & 100 & 99.0 \\
\hline Lead & 11 & .6 & .4 & .5 & .6 \\
\hline Manganese & 6 & 127 & 26.3 & 57.1 & 44.7 \\
\hline Nickel & 6 & .3 & .1 & .2 & .2 \\
\hline Zinc & 11 & 329 & 117 & 209 & 194 \\
\hline
\end{tabular}


Table 22. Statistical summary of biological data for the upper Clark Fork basin, Montana, August 1986 through August 2005. -Continued

[Concentrations are in micrograms per gram dry weight. Number of composite samples represents the total of all individual composite samples collected for every year that the constituent was analyzed. Values for a single sample are arbitraily listed in the "Mean" column. Because Hydropsyche insects were not sorted to the species level during 1986-89, Hydropsyche species statistics for stations sampled during those years are based on the results of all Hydropsyche species combined. At some sites, statistics of Hydropsyche morosa group are based on the combined results of two or more species. Insects collected during 1986-98 were depurated prior to analysis; depuration was discontinued in 1999. Arsenic data were not collected at some sites. Values are reported using U.S. Geological Survey rounding standards. Abbreviation: spp., one or more similar species. Symbols: <, less than minimum reporting level; --, indicates either too few samples (less than three) or insufficient data to compute statistic, or element not analyzed]

\begin{tabular}{|c|c|c|c|c|c|}
\hline Constituent & $\begin{array}{l}\text { Number of } \\
\text { composite samples }\end{array}$ & Maximum & Minimum & Mean & Median \\
\hline \multicolumn{6}{|c|}{$\begin{array}{l}12340000 \text {-Blackfoot River near Bonner-Continued } \\
\text { Period of record for biological data: 1986, 1987, 1991, 1993, 1996, 1998, 2000, } 2003\end{array}$} \\
\hline \multicolumn{6}{|c|}{ Hydropsyche occidentalis } \\
\hline Arsenic & 1 & -- & -- & 3.2 & -- \\
\hline Cadmium & 13 & .5 & 0.1 & .2 & 0.2 \\
\hline Chromium & 13 & 2.7 & .8 & 1.8 & 1.7 \\
\hline Copper & 13 & 20.6 & 12.0 & 14.4 & 14.4 \\
\hline Iron & 13 & 1,930 & 1,060 & 1,410 & 1,470 \\
\hline Lead & 13 & 1.9 & .8 & 1.3 & 1.3 \\
\hline Manganese & 13 & 577 & 414 & 480 & 466 \\
\hline Nickel & 13 & 1.8 & .9 & 1.3 & 1.2 \\
\hline Zinc & 13 & 150 & 116 & 135 & 130 \\
\hline \multicolumn{6}{|c|}{ Hydropsyche spp. } \\
\hline Arsenic & 0 & -- & -- & -- & -- \\
\hline Cadmium & 1 & -- & -- & 0.6 & -- \\
\hline Chromium & 1 & -- & -- & 1.6 & -- \\
\hline Copper & 1 & -- & -- & 13.9 & -- \\
\hline Iron & 1 & -- & -- & 1,140 & -- \\
\hline Lead & 1 & -- & -- & 2.9 & -- \\
\hline Manganese & 1 & -- & -- & 525 & -- \\
\hline Nickel & 1 & -- & -- & 2.8 & -- \\
\hline Zinc & 1 & -- & -- & 132 & -- \\
\hline \multicolumn{6}{|c|}{$\begin{array}{l}12340500 \text {-Clark Fork above Missoula } \\
\text { Period of record for biological data: } 1997-2005\end{array}$} \\
\hline \multicolumn{6}{|c|}{ Arctopsyche grandis } \\
\hline Arsenic & 9 & 4.5 & 2.1 & 3.3 & 3.5 \\
\hline Cadmium & 28 & 1.8 & .1 & .7 & 6 \\
\hline Chromium & 28 & 3.4 & .6 & 1.6 & 1.4 \\
\hline Copper & 28 & 77.6 & 19.5 & 33.8 & 29.1 \\
\hline Iron & 28 & 2,340 & 476 & 968 & 894 \\
\hline Lead & 28 & 6.8 & 1.2 & 3.8 & 3.3 \\
\hline Manganese & 28 & 1,410 & 476 & 950 & 937 \\
\hline Nickel & 28 & 2.0 & .5 & 1.1 & 1.0 \\
\hline Zinc & 28 & 260 & 133 & 186 & 183 \\
\hline
\end{tabular}


Table 22. Statistical summary of biological data for the upper Clark Fork basin, Montana, August 1986 through August 2005. -Continued

[Concentrations are in micrograms per gram dry weight. Number of composite samples represents the total of all individual composite samples collected for every year that the constituent was analyzed. Values for a single sample are arbitraily listed in the "Mean" column. Because Hydropsyche insects were not sorted to the species level during 1986-89, Hydropsyche species statistics for stations sampled during those years are based on the results of all Hydropsyche species combined. At some sites, statistics of Hydropsyche morosa group are based on the combined results of two or more species. Insects collected during 1986-98 were depurated prior to analysis; depuration was discontinued in 1999. Arsenic data were not collected at some sites. Values are reported using U.S. Geological Survey rounding standards. Abbreviation: spp., one or more similar species. Symbols: <, less than minimum reporting level; --, indicates either too few samples (less than three) or insufficient data to compute statistic, or element not analyzed]

\begin{tabular}{|c|c|c|c|c|c|}
\hline Constituent & $\begin{array}{c}\text { Number of } \\
\text { composite samples }\end{array}$ & Maximum & Minimum & Mean & Median \\
\hline \multicolumn{6}{|c|}{$\begin{array}{l}12340500 \text { - Clark Fork above Missoula-Continued } \\
\text { Period of record for biological data: 1997-2005 }\end{array}$} \\
\hline \multicolumn{6}{|c|}{ Claassenia sabulosa } \\
\hline Arsenic & 3 & 1.9 & 0.7 & 1.2 & 1.1 \\
\hline Cadmium & 12 & 2.0 & .2 & .7 & .4 \\
\hline Chromium & 12 & 1.1 & .3 & .7 & .7 \\
\hline Copper & 12 & 71.7 & 33.0 & 48.5 & 44.8 \\
\hline Iron & 12 & 402 & 95.3 & 245 & 241 \\
\hline Lead & 12 & 3.1 & .5 & 1.2 & 1.1 \\
\hline Manganese & 12 & 683 & 75.2 & 252 & 212 \\
\hline Nickel & 12 & $<.4$ & $<.3$ & ${ }^{1} .4$ & ${ }^{1} .4$ \\
\hline Zinc & 12 & 363 & 191 & 269 & 261 \\
\hline \multicolumn{6}{|c|}{ Hydropsyche cockerelli } \\
\hline Arsenic & 6 & 6.5 & 3.6 & 5.3 & 5.3 \\
\hline Cadmium & 15 & 1.3 & .4 & .8 & 1.0 \\
\hline Chromium & 15 & 6.0 & 1.8 & 3.1 & 3.1 \\
\hline Copper & 15 & 96.1 & 29.9 & 56.7 & 56.3 \\
\hline Iron & 15 & 3,590 & 1,400 & 2,050 & 1,900 \\
\hline Lead & 15 & 10.0 & 4.2 & 6.8 & 6.3 \\
\hline Manganese & 15 & 1,910 & 781 & 1,270 & 1,250 \\
\hline Nickel & 15 & 2.4 & 1.4 & 1.8 & 1.7 \\
\hline Zinc & 15 & 237 & 156 & 201 & 207 \\
\hline \multicolumn{6}{|c|}{ Hydropsyche occidentalis } \\
\hline Arsenic & 3 & 6.2 & 3.9 & 5.4 & 6.2 \\
\hline Cadmium & 9 & 1.2 & .4 & .7 & .7 \\
\hline Chromium & 9 & 5.5 & 2.1 & 3.4 & 3.0 \\
\hline Copper & 9 & 76.5 & 30.3 & 52.2 & 56.0 \\
\hline Iron & 9 & 2,400 & 1,450 & 2,020 & 2,210 \\
\hline Lead & 9 & 10.2 & 4.0 & 6.8 & 7.1 \\
\hline Manganese & 9 & 2,460 & 939 & 1,910 & 1,970 \\
\hline Nickel & 9 & 2.4 & 1.6 & 2.0 & 2.1 \\
\hline Zinc & 9 & 257 & 192 & 229 & 230 \\
\hline
\end{tabular}


Table 22. Statistical summary of biological data for the upper Clark Fork basin, Montana, August 1986 through August 2005. -Continued

[Concentrations are in micrograms per gram dry weight. Number of composite samples represents the total of all individual composite samples collected for every year that the constituent was analyzed. Values for a single sample are arbitraily listed in the "Mean" column. Because Hydropsyche insects were not sorted to the species level during 1986-89, Hydropsyche species statistics for stations sampled during those years are based on the results of all Hydropsyche species combined. At some sites, statistics of Hydropsyche morosa group are based on the combined results of two or more species. Insects collected during 1986-98 were depurated prior to analysis; depuration was discontinued in 1999. Arsenic data were not collected at some sites. Values are reported using U.S. Geological Survey rounding standards. Abbreviation: spp., one or more similar species. Symbols: <, less than minimum reporting level; --, indicates either too few samples (less than three) or insufficient data to compute statistic, or element not analyzed]

\begin{tabular}{|c|c|c|c|c|c|}
\hline Constituent & $\begin{array}{c}\text { Number of } \\
\text { composite samples }\end{array}$ & Maximum & Minimum & Mean & Median \\
\hline \multicolumn{6}{|c|}{$\begin{array}{c}12353000 \text {-Clark Fork below Missoula² } \\
\text { Period of record for biological data: 1986; 1990-2004 }\end{array}$} \\
\hline \multicolumn{6}{|c|}{ Arctopsyche grandis } \\
\hline Arsenic & 4 & 2.6 & 2.1 & 2.4 & 2.4 \\
\hline Cadmium & 29 & 1.5 & .2 & .6 & .6 \\
\hline Chromium & 29 & 2.7 & .5 & 1.3 & 1.4 \\
\hline Copper & 29 & 38.0 & 9.4 & 21.1 & 19.2 \\
\hline Iron & 29 & 1,590 & 343 & 797 & 682 \\
\hline Lead & 29 & 3.9 & .9 & 1.9 & 1.7 \\
\hline Manganese & 29 & 1,090 & 511 & 707 & 692 \\
\hline Nickel & 29 & 1.6 & .4 & .9 & .9 \\
\hline Zinc & 29 & 217 & 106 & 152 & 148 \\
\hline \multicolumn{6}{|c|}{ Claassenia sabulosa } \\
\hline Arsenic & 3 & 1.1 & 0.8 & 0.9 & 0.8 \\
\hline Cadmium & 42 & 1.3 & .1 & .5 & .4 \\
\hline Chromium & 42 & 1.2 & .05 & .5 & .5 \\
\hline Copper & 42 & 75.1 & 31.1 & 48.8 & 47.0 \\
\hline Iron & 42 & 239 & 66.6 & 114 & 108 \\
\hline Lead & 42 & 1.3 & .1 & .4 & .3 \\
\hline Manganese & 42 & 275 & 48.9 & 107 & 99 \\
\hline Nickel & 42 & .3 & .1 & .2 & .2 \\
\hline Zinc & 42 & 324 & 146 & 218 & 211 \\
\hline \multicolumn{6}{|c|}{ Hydropsyche cockerelli } \\
\hline Arsenic & 8 & 2.9 & 2.2 & 2.5 & 2.4 \\
\hline Cadmium & 46 & 1.1 & .2 & .5 & .5 \\
\hline Chromium & 46 & 3.4 & .3 & 1.7 & 1.7 \\
\hline Copper & 46 & 54.1 & 12.4 & 30.4 & 29.5 \\
\hline Iron & 46 & 2,220 & 584 & 1,290 & 1,270 \\
\hline Lead & 46 & 6.6 & 1.2 & 2.5 & 2.4 \\
\hline Manganese & 46 & 1,210 & 353 & 768 & 690 \\
\hline Nickel & 46 & 1.9 & .5 & 1.2 & 1.3 \\
\hline Zinc & 46 & 187 & 77 & 144 & 151 \\
\hline
\end{tabular}


Table 22. Statistical summary of biological data for the upper Clark Fork basin, Montana, August 1986 through August 2005. -Continued

[Concentrations are in micrograms per gram dry weight. Number of composite samples represents the total of all individual composite samples collected for every year that the constituent was analyzed. Values for a single sample are arbitraily listed in the "Mean" column. Because Hydropsyche insects were not sorted to the species level during 1986-89, Hydropsyche species statistics for stations sampled during those years are based on the results of all Hydropsyche species combined. At some sites, statistics of Hydropsyche morosa group are based on the combined results of two or more species. Insects collected during 1986-98 were depurated prior to analysis; depuration was discontinued in 1999. Arsenic data were not collected at some sites. Values are reported using U.S. Geological Survey rounding standards. Abbreviation: spp., one or more similar species. Symbols: <, less than minimum reporting level; --, indicates either too few samples (less than three) or insufficient data to compute statistic, or element not analyzed]

\begin{tabular}{|c|c|c|c|c|c|}
\hline Constituent & $\begin{array}{c}\text { Number of } \\
\text { composite samples }\end{array}$ & Maximum & Minimum & Mean & Median \\
\hline \multicolumn{6}{|c|}{$\begin{array}{l}12353000 \text {-Clark Fork below Missoula²_Continued } \\
\text { Period of record for biological data: 1986; 1990-2004 }\end{array}$} \\
\hline \multicolumn{6}{|c|}{ Hydropsyche occidentalis } \\
\hline Arsenic & 1 & -- & -- & 2.5 & -- \\
\hline Cadmium & 17 & 1.1 & 0.1 & .4 & 0.3 \\
\hline Chromium & 17 & 3.5 & .1 & 1.4 & 1.5 \\
\hline Copper & 17 & 38.2 & 13.5 & 23.8 & 20.9 \\
\hline Iron & 17 & 1,420 & 482 & 941 & 907 \\
\hline Lead & 17 & 4.2 & .7 & 2.1 & 1.9 \\
\hline Manganese & 17 & 1,460 & 491 & 841 & 812 \\
\hline Nickel & 17 & 2.2 & .5 & 1.0 & .9 \\
\hline Zinc & 17 & 193 & 112 & 143 & 144 \\
\hline \multicolumn{6}{|c|}{ Hydropsyche spp. } \\
\hline Arsenic & 0 & -- & -- & -- & -- \\
\hline Cadmium & 1 & -- & -- & 0.5 & -- \\
\hline Chromium & 1 & -- & -- & .8 & -- \\
\hline Copper & 1 & -- & -- & 20.8 & -- \\
\hline Iron & 1 & -- & -- & 894 & -- \\
\hline Lead & 1 & -- & -- & 1.1 & -- \\
\hline Manganese & 1 & -- & -- & 756 & -- \\
\hline Nickel & 1 & -- & -- & 1.1 & -- \\
\hline Zinc & 1 & -- & -- & 124 & -- \\
\hline
\end{tabular}

${ }^{1}$ Values were determined by substituting one-half of the minimum reporting level for censored $(<)$ values when both uncensored and censored values were used in determining the mean and median. When all data were less than the minimum reporting level, the median was determined by ranking the censored values in order of detection. No mean is reported when all values were below the minimum reporting level.

${ }^{2}$ Samples were collected about 30 miles downstream from streamflow-gaging station to conform to previous sampling location. 
For additional information contact:

Director, Montana Water Science Center

U.S. Geological Survey

3162 Bozeman Avenue

Helena, Montana 59601

Telephone: 1-406-457-5900

World Wide Web: http://mt.water.usgs.gov/ 
